\title{
El Problema de Sitnikov con 4-Cuerpos
}

Tesis para obtener el grado de Doctor en Ciencias (Matemáticas)

Presentado por el Mat. Hugo Jiménez Pérez

Miércoles 13 de enero de 2010

Director de tesis:

Dr. Ernesto Alejandro Lacomba Zamora.

Miembros del jurado:

Dr. Arturo Olvera Chávez - Presidente.

Dr. Ernesto Alejandro Lacomba Zamora - Secretario.

Dr. Ernesto Pérez Chavela - Vocal.

Dr. Jesús Muciño Raymundo - Vocal.

Dr. Antonio Hernández Garduño - Vocal. 
A mi esposa Diane y a mi hiJa Iris.

EN LA ETERNIDAD DE NUESTROS SUEÑOS SEGUIREMOS JUNTOS TODA LA VIDA... 



\section{ÍNDICE GENERAL}

Introducción

Agradecimientos XI

Capítulo 1. Antecedentes 1

1.1 Espacios simplécticos. 1

1.2 Variedades simplécticas. 5

1.3 Sistemas Hamiltonianos. 12

1.4 Acciones Hamiltonianas v la aplicación momento. 16

1.5 Reducción de sistemas Hamiltonianos con simetría. 19

1.6 Funciones generatrices. 20

1.7 Sistemas mecánicos. 24

1.8 El problema de los $N$ cuerpos. 27

1.9 Singularidades en el problema de $N$-cuerpos. 28

1.10 Reducción simpléctica del problema de $N$ cuerpos. 31

Capítulo 2. El problema de Sitnikov con $2+2$ cuerpos 37

2.1 El problema de Sitnikov. 37

2.2 El problema de Sitnikov con $2+2$ cuerpos. 40

2.3 Regularización de colisiones. 43

2.4 La transformación de Euler. 46

2.5 Regularización del problema circular 2+2 de Sitnikov 49

Capítulo 3. Estudio del caso restringide 57

3.1 El problema circular restringido de Sitnikov con $2+2$ cuerpos. 57

3.2 Solución analítica del caso circular restringido. 58

3.3 Hiper-superficies de energía constante. 66

3.4 El conjunto de colisión.

3.5 Transferencia de momento. 82

3.6 Soluciones periódicas en el problema circular: $m_{3}=m_{1}$. 87

Capítulo 4. Continuación de órbitas periódicas 95 
4.1 Continuación de órbitas simétricas 95

4.2 Cadenas de orden 1 en el problema 2+2 100

4.3 Cadenas de orden mavor y relación $n: m$

\begin{tabular}{lll}
\hline Capítulo 5. Conclusiones v perspectivas. & 113
\end{tabular}

5.1 Regularización de colisiones. 113

5.2 Coordenadas acción-ángulo v solución analítica. 114

$\begin{array}{ll}5.3 \text { Transferencia de momento. } & 115\end{array}$

5.4 Foliación del espacio fase. 116

5.5 Continuación de óbitas periódicas. 116

5.6 Nuevas posibilidades de investigación. 117

\begin{tabular}{lll}
\hline Apéndice A. Grupos v álgebras de Lid & 123
\end{tabular}

A.1 Grupos de Lie. 123

A.2 Álgebras de Lie. 125

A.3 Álgebras de Lie de grupos de Lie. 127

A.4 Subgrupos a un parámetro v la aplicación exponencial. 128

$\begin{array}{ll}\text { A.5 Derivada de Lie. } & 129\end{array}$

\begin{tabular}{|lll}
\hline Apéndice B. Formas diferenciales & 133
\end{tabular}

B.1 Aplicaciones multilineales alternadas. 133

B.2 Formas diferenciales. 134

\begin{tabular}{|lll}
\hline Apéndice C. Funciones e integrales elípticas & 141
\end{tabular}

C.1 Integrales elípticas. 141

$\begin{array}{ll}\text { C.2 Funciones elípticas. } & 144\end{array}$

C.3 Funciones elípticas de Jacobi. 145

$\begin{array}{ll}\text { C.4 Argumento imaginario. } & 147\end{array}$

C.5 Derivadas de las funciones elípticas de Jacobi. 148

$\begin{array}{ll}\text { C.6 Teorema de la adición. } & 148\end{array}$

$\begin{array}{ll}\text { C.7 Periodicidad y polos. } & 149\end{array}$

C.8 Transformaciones del módulo. 154

\begin{tabular}{ll}
\hline Bibliografía & 157
\end{tabular}

$\begin{array}{ll}\text { Indice alfabético } & 163\end{array}$ 


\section{INTRODUCCIÓN}

La mecánica celeste es un tema que tiene una larga y diversa historia. A través de muchos años, tanto filósofos como científicos han tratado de responder a una serie de preguntas acerca de la dinámica del sistema solar y particularmente de su estabilidad. De manera simple podemos decir que la mecánica celeste es el estudio de la solución de un sistema de ecuaciones diferenciales, conocido como el problema de $N$-cuerpos. Este problema está relacionado con el movimiento de $N$ masas puntuales que se atraen unas a otras bajo las fuerzas gravitacionales Newtonianas, en el espacio físico tridimensional.

En este trabajo se presenta un problema restringido de 4 cuerpos en una configuración original que presenta muchas simetrías. La configuración con 4-cuerpos es la siguiente: dos cuerpos de masas $m_{1}=m_{2}=1 / 2$ evolucionan en el plano horizontal $X Y$ describiendo órbitas Keplerianas y dos cuerpos infinitesimales $m_{3} \approx m_{4} \approx 0$ moviéndose en el eje $Z$ sin ejercer atracción sobre los primarios. Esta configuración es una generalización del problema de Sitnikov que se obtiene al agregar un segundo cuerpo infinitesimal en la recta vertical de dicho problema. De esta forma, el problema será llamado el Problema de Sitnikov con 2+2 cuerpos.

Las aportaciones de este trabajo de investigación son las siguientes:

- Presentación de un problema restringido de 4 cuerpos que permite un estudio detallado de la interacción entre los cuerpos infinitesimales.

- Estudio del proceso de regularización para colisiones binarias colineales en un sistema que presenta fuerzas externas.

- Obtención de las coordenadas acción-ángulo para el problema circular restringido de Sitnikov con $2+2$ cuerpos. Método alternativo para encontrar las soluciones analíticas.

- Aplicación de técnicas de la transferencia de momento en un problema de mecánica celeste. Es de gran importancia notar que la existencia de colisiones binarias en un sistema con fuerzas ajenas a los cuerpos que colisionan no permite un cambio de coordenadas 
donde el conjunto de colisiones se reduzca al centro de masa del sistema.

- Uso de la transferencia de momento para continuación de órbitas periódicas cuando no existe simetría en las superficies de energía constante de un sistema mecánico.

- Clasificación de la dinámica del problema circular restringido de Sitnikov de $2+2$ cuerpos.

RESEÑA HISTÓRICA. El problema de Sitnikov es un caso particular del problema restringido de tres cuerpos, cuyas soluciones muestran una dinámica muy diversa. De hecho, en un estudio realizado por el astrónomo francés J. Chazy sobre las evoluciones finales del problema restringido de tres cuerpos (1922-1931 [20]), incluyó en su clasificación un tipo de movimientos que llamó oscilatorios, sin tener conocimiento de alguna configuración que pudiera presentar estos movimientos.

En 1954, Kolmogorov propuso una configuración del problema de tres cuerpos en la cual se tenían dos cuerpos de masas iguales $m_{1}=m_{2}$ en el plano $X Y$, situados de manera simétrica con respecto del eje vertical $Z$ y un tercer cuerpo de masa $m_{3}$ sobre la recta vertical con velocidad en dirección vertical también. Los cuerpos de masas $m_{1}$ y $m_{2}$ tendrían así una evolución en órbitas simétricas (no cerradas en general) y el tercer cuerpo permanecería sobre la vertical debido a la simetría del problema [2] Esta configuración, con la restricción que $m_{3}=0$, le permitió a Sitnikov demostrar la existencia de órbitas oscilatorias en el problema restringido de tres cuerpos [94, 1960].

Cuando las órbitas de los cuerpos masivos son circulares, las ecuaciones que describen el movimiento del cuerpos infinitesimal no dependen del tiempo físico explícitamente. Esto reduce las dimensiones del sistema de ecuaciones y permite su integración explícita. Este caso, ya se había estudiado por Pavinini en 1907 y también por Macmillan en 1911 [65, quien presentó la solución en términos de funciones e integrales elípticas y su aproximación mediante series de funciones trigonométricas. Esto permitió hacer cálculos explícitos y durante muchos años se conoció como el problema de MacMillan. Actualmente se conoce como el problema circular de Sitnikov.

En la decada de los 60s, el problema adquirió mucho interés entre físicos, astrónomos y matemáticos. Alekseev presentó un estudio detallado sobre sistemas dinámicos, ecuaciones diferenciales y dinámicas simbólicas y utilizó como principal ejemplo la configuración de Sitnikov [2]. Una afirmación importante en dicho trabajo (en el segundo y tercer artículo) es que en el caso en que la masa del tercer cuerpo es positiva $m_{3}>0$, la dinámica del problema es equivalente al caso $m_{3}=0$, en el sentido que se presenta un conjunto hiperbólico invariante donde se puede sumergir un corrimiento de Bernoulli. En 1973, Moser presenta una obra donde simplifica los trabajos de Alekseev utilizando dinámicas simbólicas [77] y demuestra la no integrabilidad del problema de Sitnikov en general. A partir de ese momento, decenas de científicos han trabajado sobre este problema, entre los que podemos 
mencionar (sin un orden en particular) a Llibre, Simó, Xia, Dankowicz, Holmes, Belbruno, Ollé, García-Rodríguez, Pérez-Chavela, Chesley, Lacomba, Corbera, Jie Liu, Yi-Sui Sun, Robinson, Dvorak, Fernández-Hevia, Rañada, Jalali, Pourtakdoust, entre muchos otros. Algunas generalizaciones de este problema incluyen las siguientes:

- El problema de Sitnikov con tres masas iguales propuesto por Dvorak [30, 1993].

- El problema de Sitnikov en $\mathbb{R}^{4}$ propuesto por Lacomba, Llibre y Pérez-Chavela [57, 2002].

- El problema circular de Sitnikov con 4-cuerpos en una configuración $3+1$ propuesto por Soulis, Papadakis y Bountis [95. 2008].

- El problema circular de Sitnikov con $2+2$ cuerpos propuesto en este trabajo de investigación [49, 2009].

ACERCA DEL PRESEnTE DoCumento. Este trabajo detalla el problema de Sitnikov con $2+2$ cuerpos en el caso restringido circular y explora el caso en que las masas de los secundarios tienden a cero con una razón lineal $m_{4}=c m_{3}$ para $0<c \leq 1$. En particular se puso mucha atención al estudio del problema con intercambio de momento que sufren los cuerpos secundarios durante una colisión. Este caso llega a ser de mucho interés en mecánica celeste y astrodinámica para aplicaciones inmediatas a problemas de satelites y asteroides.

Cuando se tienen masas iguales en los secundarios $(c=1)$ no existe transferencia de momento durante las colisiones. En este caso se encuentran las condiciones bajo las cuales se tienen órbitas periódicas y posteriormente se buscan las condiciones para generar familias de órbitas periódicas que se puedan extender al caso $c \neq 1$ considerando el intercambio de momento durante la colisión.

Cuando se tienen masas diferentes en los secundarios $(0<c<1)$ el comportamiento cambia. Para continuar una solución después de una colisión, si esta se presenta fuera del origen de coordenadas, la transferencia de momento genera una discontinuidad en el espacio fase. Esto se debe a que la proyección de las superficies de nivel y de los conjuntos de colisión en el espacio de momentos son elipses en lugar de circunferencias y la simetría con respecto de las rectas (diagonales) que determinan el intercambio de momento se ha perdido.

Otra dificultad es que el proceso de regularización reduce la dimensión del conjunto de colisiones creando una transformación cuyo rango no es completo en dicho conjunto. Al revertir el proceso debemos determinar la forma de "pegar" las soluciones y esto se deberá hacer solución a solución. Este comportamiento se utiliza en este trabajo para hacer la continuación de órbitas periódicas.

A continuación se da un bosquejo general del documento: En el Capítulo 1, se presentan los antecedentes relacionados con la geometría simpléctica, sistemas Hamiltonianos, y sus aplicaciones al problema de $N$-cuerpos. El 
Capítulo 2 está dedicado a la descripción de nuestro problema. Se inicia con la exposición del problema de Sitnikov clásico y posteriormente se presenta el Problema de Sitnikov con $2+2$ cuerpos, sus ecuaciones de movimiento y una clasificación basada en los valores de los parámetros que aparecen en las ecuaciones. En el mismo capítulo se presenta la regularización de las colisiones del problema y finalizamos con un caso particular con condiciones iniciales simétricas que llamamos el problema circular simétrico de Sitnikov con $2+2$ cuerpos; se demuestra que este caso es integrable por cuadraturas y se determina la dinámica de los cuerpos secundarios.

En el Capítulo 3 se hace un estudio del problema circular cuando no existe interacción entre los secundarios. Este caso lo llamaremos el problema circular restringido de Sitnikov con $2+2$ cuerpos. Al ser un caso restringido, las ecuaciones de movimiento se desacoplan y obtenemos un problema integrable de 2 grados de libertad. Posteriormente se obtienen las coordenadas acción-ángulo, las soluciones analíticas y la foliación del espacio fase por subvariedades Lagrangianas. También se hace un estudio del conjunto de colisiones que resulta ser una 3 -variedad foliada por esferas $\mathbb{S}^{2}$ y cilindros $\mathbb{S}^{1} \times \mathbb{R}$. (Esta variedad de colisión no coincide con el concepto clásico definido por McGehee [70 y otros autores.) Se inicia un estudio sobre la transferencia de momento y se caracteriza la forma de "pegar" las soluciones después de una colisión. Finalizamos con la caracterización de las órbitas periódicas sobre toros Lagrangianos resonantes para el caso $m_{3}=m_{4}$.

El Capítulo 4 está dedicado a extender las órbitas periódicas simétricas que se encuentran en el caso con $m_{3}=m_{4}$ a órbitas periódicas para pequeñas variaciones de las masas $m_{3} \neq m_{4}$. Este método de continuación es equivalente al método de continuación de Poincaré para órbitas periódicas de segunda especie, sin embargo, aquí se utiliza la transferencia de momento y el rebote elástico para obtener las nuevas órbitas periódicas. El Capítulo 5 está reservado para algunos problemas abiertos que han surgido durante la investigación y para establecer nuestras conclusiones. Adicionalmente se tienen tres Apéndices: el primero da antecedentes de Formas diferenciales que se utiliza para algunos resultados sobre simplectomorfismos. El segundo apéndice da los antecedentes de Grupos y álgebras de Lie que se necesitan para entender otros conceptos adicionales sobre reducción simpléctica, acciones Hamiltonianas y del mapeo momento. El tercer y último apéndice es una breve intoducción a la teoría de funciones e integrales elípticas de Jacobi.

El problema se aborda como un problema integrable y se utiliza la teoría de sistemas Hamiltonianos completamente integrables para comprender la geometría y la topología de las subvariedades donde se encuentran las soluciones. El caso integrable corresponde al problema circular restringido de Sitnikov con $2+2$ cuerpos. En particular, se utiliza herramienta de la geometría simpléctica y teoría de Lie, específicamente de álgebras de Lie y acciones Hamiltonianas sobre variedades simplécticas. 
Algunos resultados son generales, como la regularización de las ecuaciones del problema. Bajo la misma técnica se puede regularizar toda configuración de $n+2$ cuerpos con $n$ primarios en el plano en equilibrio relativo poligonal y los secundarios evolucionan en la recta perpendicular que pasa por el centro del polígono. Asimismo, se puede utilizar para los problemas de $2 n+2$ cuerpos donde se tienen 2 polígonos regulares anidados con $n$ cuerpos en sus vértices y los secundarios que se mueven en la recta perpendicular que pasa por el centro de masas del sistema primario. 



\section{Agradecimientos}

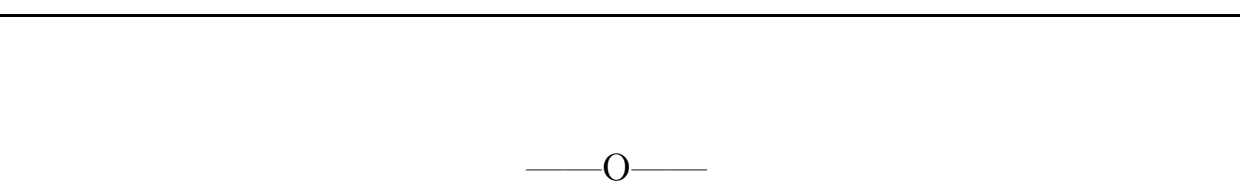

Agradezco al CoNaCyT por el apoyo ecónomico para realizar mis estudios doctorales a través de la beca número 184728, incluyendo la estancia de investigación en el Observatorio de Paris. Agradezco también al Institut de Mécanique Céleste et Calcul des Ephémerides (IMCCE) del Observatorio de Paris por acogerme como investigador visitante durante mi investigación doctoral.

Agradezco a mi asesor, Ernesto Lacomba, por iniciarme en el estudio del problema de Sitnikov y por la sugerencia del problema con 4 cuerpos que dió origen a este proyecto doctoral. También le agradezco su confianza, su ayuda y por acercarme al estudio de la mecánica celeste desde una perspectiva geométrica.

Agradezco a mis sinodales del examen doctoral: Arturo Olvera, Ernesto Pérez Chavela, Jesús Muciño y Antonio Hernández por su ayuda y el tiempo dedicado a revisar y mejorar este trabajo.

Agradezco a mis sinodales del examen predoctoral: Manuel Falconi, Lidia Jiménez y Antonio García por las ideas aportadas en el planteamiento de este proyecto.

$\mathrm{O}$

Esta sección es de mucha ayuda moral ya que me permitió hacer un pequeño flashback y comparar lo que sabía antes de iniciar la tesis con lo que sé al momento de terminarla. El aspecto más importante es resaltar toda la ayuda que he recibido durante estos difíciles años y la capacidad que tienen mis colegas para compartir sus ideas y su conocimiento.

Primero quiero agradecer a mi familia por todo el tiempo que me han dado para hacer mis estudios, mi investigación y las asistencias a congresos y conferencias, para mi hija Iris y mi esposa Diane con todo mi corazón. También deseo agradecer a mi madre Carmen, a Blanca Rosa Pérez, a Rosendo Castillo Pérez y Armando Castillo Ánimas por su apoyo incondicional.

La siguiente lista no tiene un orden y no es exhaustiva, pero si olvido a alguien, espero que no se tome de manera personal. Deseo agradecer a Santiago López de Medrano por la confianza con la que siempre me ha 
recibido, la ayuda y las sesiones informales. Asimismo deseo agradecer a Laurent Niederman por haberme acogido en el observatorio de Paris y haber fungido como asesor externo en mi estancia en el IMCCE. Al equipo de mecánica celeste de la UAM-I que me apoyaron durante toda esta aventura: Martha Alvarez (primero las damas :) , Joaquín Delgado, Ernesto PérezChavela, Antonio García, Luis Aguirre, Mario Medina y a mis compañeros de estudios Luis Franco por todos los dolores de cabeza que pasó escuchando mis locas ideas, a José Luis Cosme por todas las vueltas a la división de CBI para hacer mi papeleo y a Ismael Velázquez por su confianza.

Quiero agredecer particularmente a las personas que aportaron ideas en relación a la investigación que se realizó... algunos de ellos creo que no sabrán siquiera que tuvieron algo que ver. Inicio con Jesús Muciño, que me pidió apropiarme de mi trabajo de tesis, a Gerardo Hernández que aportó preguntas valiosas, a Claudio Vidal por las sesiones y consejos sobre teorema de Arenstorf, a Alexander Bruno por sus comentarios sobre el estudio del problema con cadenas a través de colisiones, a J-P Marco por ideas sobre la importancia de hacer continuación a órbitas sin colisiones, a Federiqua Pasquotto por explicarme su trabajo sobre órbitas periódicas en sistemas mecánicos, a Jaume Llibre por las sesiones que dedicó a escuchar mi trabajo, sus comentarios e ideas sobre órbitas de segunda especie, a Jacques Fejoz por la sencillez de sus conversaciones y algunas ideas simples pero efectivas, a Alain Chenciner por las palabras de aliento y por darme los primeros textos de teoría de Ramis-Morales y de su trabajo con Emmanuelle Tossier, a Primitivo Acosta porque me dijo que no lo olvidara al escribir mis agradecimientos (y por supuesto por los cursillos del algoritmo de Kovacic en la teoría de Ramis-Morales), a Carlos Signoret por los seminarios de álgebra homológica, Andrés Pedroza por iniciarme en la teoría de acciones Hamiltonianas.

A mis colegas y compañeros de posgrado que siempre me escuchaban sufriendo porque muchas veces no entendían lo que yo tampoco entendía, pero al platicárselo empezaba a tomar forma en mi cabeza. A los profesores que me dieron cursos y también a aquellos que no lo hicieron pero me dedicaron algún tiempo para explicar detalles valiosos: Rogelio Fernández, Laura Hidalgo, Horacio Tapia, Guadalupe Reyes.

Y para todos los demás que aportaron algún elemento adicional en este trabajo pero que ahora no recuerdo, también les doy mi agradecimiento... ¡Ah! faltan también Oscar Palmas por detalles sobre álgebras de Lie y Guillermo Gómez por discusiones sobre billares y colisiones. 


\section{CAPÍTULO 1}

\section{ANTECEDENTES}

El objetivo de este capítulo es presentar los fundamentos de los sistemas Hamiltonianos y de la geometría simpléctica que son necesarios para comprender el contenido de este trabajo. Sólo se incluirán los resultados más relevantes de dicha teoría con la finalidad de que el trabajo sea autocontenido.

Para una introducción más detallada a la dinámica de los sistemas Hamiltonianos y la geometría simpléctica, recomendamos al lector consultar los textos de Abraham y Marsden [1 y el excelente libro de Arnold 4] que son dos de las referencias más completas. También se recomienda el texto de Meyer [74 para sistemas Hamiltonianos y su aplicación al problema de N-cuerpos, Cannas da Silva 15, Audin [5], y por supuesto Hoffer y Zender 43 para geometría Hamiltoniana y geometría simpléctica y el libro de MacDuff 69] para topología simpléctica. Para la sección de redución simpléctica se pueden revisar los textos de Berndt [9, Perelomov 83] y Mircea Puta 87. En particular, el último es un texto excelente que cubre muchos de los casos que se presentan en los sistemas mecánicos.

\subsection{EsPACIOS SIMPLÉCtiCOS.}

Iniciemos este capítulo con un punto de vista geométrico y que consiste en obtener los sistemas Hamiltonianos como funciones definidas sobre variedades simplécticas exactas y donde el campo vectorial se obtiene de manera natural mediante la 2 -forma diferencial $\omega$ que le da la estructura simpléctica a la variedad.

1.1.1. Espacios VECTORIALES Simplécticos. La noción de espacio vectorial simpléctico se basa en proveer a un espacio vectorial lineal con una 
estructura simpléctica, proporcionada por una 2-forma diferencial conocida como forma simpléctica.

DeFinición 1.1. Sea E un espacio vectorial lineal sobre el campo $\mathbb{R}$. Una forma simpléctica $\omega_{0}$ sobre el espacio $E$ es una forma diferencial que cumple las condiciones

- $\omega$ es bilineal,

- antisimétrica,

- y no degenerada.

Definición 1.2. Sea $E$ un espacio vectorial de dimensión finita sobre $\mathbb{R}$ y sea $\omega: E \times E \rightarrow \mathbb{R}$ una forma bilineal antisimétrica no degenerada. A la pareja $(E, \omega)$ se le llama un espacio vectorial simpléctico sobre $\mathbb{R}$.

Definición 1.3. La 2-forma diferencial

$$
\omega_{0}=\sum_{i} d p_{i} \wedge d q_{i}
$$

se denomina la forma simpléctica canónica del espacio vectorial E.

Visto como un producto interno, la forma simpléctica canónica se puede escribir de la siguiente manera

$$
\omega_{0}(u, v)=\langle u, J v\rangle, \quad u, v \in E,
$$

donde $J$ es la matriz simpléctica canónica de dimensión $2 n$ definida por

$$
J=\left(\begin{array}{cc}
0_{n} & I_{n} \\
-I_{n} & 0_{n}
\end{array}\right)
$$

donde $0_{n}, I_{n} \in M_{n \times n}$ son las matrices cero e identidad respectivamente. El operador $\langle\cdot, \cdot\rangle$ es el producto interno usual en el espacio vectorial $E$.

Es evidente que $J$ es una matriz antisimétrica, pero también es una matriz ortogonal, i.e., $J J^{T}=I_{2 n}$. Por ejemplo, en el caso $n=1$ tenemos

$$
J=\left(\begin{array}{cc}
0 & 1 \\
-1 & 0
\end{array}\right)
$$

que corresponde a una rotación por $\pi / 2$ del espacio $T^{*} \mathbb{R}$.

Del hecho que $J J^{T}=I_{2 n}$ se desprende inmediatamente que $J^{-1}=J^{T}$ además, $J^{2}=-I$ por lo tanto $J^{T}=-J$.

\subsubsection{Subespacios de un espacio SimpléCtico.}

DeFinición 1.4. Un subespacio vectorial $W$ de un espacio simpléctico $E$, es un subconjunto $W \subset E$ tal que $W$ es un espacio vectorial.

Aunque la definición de subespacio vectorial es equivalente a la de los espacios vectoriales comunes, en un espacio vectorial simpléctico existen distintos tipos de subespacios y para ello se requiere el concepto de ortogonalidad simpléctica 
Definición 1.5. Sean $u, v \in E$ dos vectores en un espacio simpléctico. Diremos que $u$ es $\omega$-ortogona 1 a $v$ o que $u$ y $v$ son $\omega$-ortogonales si se cumple que

$$
\omega(u, v)=0 .
$$

Cuando haya riesgo de confusión, utilizaremos la notación $u \perp^{\omega} v$ para denotar dos vectores $\omega$-ortogonales $\mathrm{u}$ ortogonales con respecto de la forma simpléctica $\omega$.

Definición 1.6. Sea $W \subset E$ un subespacio vectorial del espacio simpléctico E. El subespacio $\omega$-ortogonal a $W$ es el conjunto

$$
W^{\omega}=\left\{x \in E \mid \omega_{0}(x, y)=0, \forall y \in W\right\} .
$$

$W^{\omega}$ es claramente un subespacio vectorial.

Como $u \perp^{\omega} v$ es equivalente a $v \perp^{\omega} u$, vemos que $\left(W^{\omega}\right)^{\omega}=W$. Tomando en cuenta que la forma $\omega$ es no degenerada, tenemos la siguiente propiedad

Proposición 1.1. Sea $W \subset E$ un subespacio vectorial del espacio simpléctico E entonces se cumple

$$
\operatorname{dim} W+\operatorname{dim} W^{\omega}=\operatorname{dim} E .
$$

Demostración. Consideremos el espacio $\left(E, \omega_{0}\right)$ con la forma simpléctica canónica

$$
\omega_{0}(v, w)=\langle v, J w\rangle
$$

en $\mathbb{R}^{2 n}$.

Si $v \in W$ y $w \in W^{\omega}$, entonces $\langle v, J w\rangle=0$, por lo tanto, $v$ es ortogonal a $J w$ en el sentido clásico de la ortogonalidad definida por $\langle$,$\rangle . El conjunto$ $\left\{J w \mid w \in W^{\omega}\right\}$ nos genera un subespacio de dimensión $2 n-\operatorname{dim} W$. Si aplicamos $J^{-1}=J^{T}$ para obtener $W^{\omega}$, la dimensión del nuevo subespacio es también $2 n-\operatorname{dim} W$. Esto nos indica finalmente que

$$
\operatorname{dim} W^{\omega}=2 n-\operatorname{dim} W .
$$

A diferencia de los subespacios ortogonales bajo el producto interno usual $\langle\cdot, \cdot\rangle$ donde $W \cap W^{\perp}=\{0\}$, la ortogonalidad con respecto a una forma simpléctica genera un subespacio vectorial $W^{\omega}$ que no siempre es complementario al subespacio $W$ y por lo tanto se puede dar que la intersección $W \cap W^{\omega}=V$ sea otro subespacio vectorial. Los subespacios vectoriales de un espacio simpléctico se clasifican de la siguiente manera

Definición 1.7. Sea $W \subset E$ un subespacio vectorial del espacio simpléctico E y sea $W^{\omega}$ el subespacio $\omega$-ortogonal a $W$, entonces $W$ se dice

1. isotrópico si $W \subset W^{\omega}$,

\footnotetext{
${ }^{1}$ En diversos textos se utiliza el término antiortogonal haciendo referencia a la terminología inglesa skew-orthogonal
} 
2. coisotrópico si $W^{\omega} \subset W$,

3. lagrangiano si $W^{\omega}=W$,

4. simpléctico si $W \cap W^{\omega}=\{0\}$.

Proposición 1.2. Los subespacios vectoriales de un espacio simpléctico cumplen las siguientes propiedades

1. W es isotrópico si y sólo si $W^{\omega}$ es coisotrópico.

2. W es coisotrópico si y sólo si $W^{\omega}$ es isotrópico.

3. Si $\operatorname{dim} W=n$ y $W$ es isotrópico, entonces $W$ es lagrangiano.

Nota 1.1. Nótese que un subespacio lagrangiano es isotrópico y coisotrópico al mismo tiempo.

Los subespacios lagrangianos cumplen con ser subespacios isotrópicos maximales y subespacios coisotrópicos minimales, en el sentido que la dimensión de un espacio isotrópico está acotado por $0 \leq \operatorname{dim} W \leq n$ y la de un espacio coisotrópico cumple con $n \leq \operatorname{dim} W \leq 2 n$.

Es posible restringir la forma bilineal $\omega(\cdot, \cdot)$ a un subespacio $W \subset E$ pero la restricción en general va a ser degenerada. La restricción es no degenerada si y sólo si $W \cap W^{\omega}=\{0\}$. Esto es equivalente a decir que

$$
W \oplus W^{\omega}=E
$$

y también a decir que $W$ es un subespacio simpléctico

Proposición 1.3. Las siguientes afirmaciones son equivalentes

1. $W \cap W^{\omega}=\{0\}$,

2. $W \oplus W^{\omega}=E$,

3. $W$ es un subespacio simpléctico,

4. $\left.\omega\right|_{W}$ es inyectiva.

1.1.3. Los Fibrados tangente y COTANGEnte. Los espacios naturales donde se trabajan los sistemas mecánicos han sido muy importantes para el nacimiento y desarrollo de la geometría simpléctica y Hamiltoniana. De hecho, el primer ejemplo de espacio vectorial simpléctico es el espacio fase de un sistema Hamiltoniano que corresponde a un objeto geométrico conocido como el fibrado cotangente. Por otra parte, los sistemas Lagrangiano 2 utilizan como espacio fase el fibrado tangente y aunque se le puede dotar de una estructura simpléctica, dicha estructura no es natural.

DEFINICIÓN 1.8. El fibrado tangente $o$ haz tangente de un espacio vectorial $W$ es el conjunto definido por

$$
T W=\left\{(q, v) ; q \in W, v \in T_{q} W\right\},
$$

donde $q$ es un punto del espacio vectorial y $v$ es un vector sobre el plano tangente $T_{q} W$ en el punto $q$.

\footnotetext{
${ }^{2}$ Reciben ese nombre porque las ecuaciones de movimiento cumplen con las ecuaciones de Euler-Lagrange y no tiene relación con la definición de subespacios Lagrangianos
} 
Es importante notar que en la definición anterior, $T_{x} W \cong W$, sin embargo, esta definición se utiliza en general cuando $W$ es una variedad diferenciable. Si $W=\mathbb{R}^{n}$ entonces $T \mathbb{R}^{n} \cong \mathbb{R}^{n} \times \mathbb{R}^{n} \cong \mathbb{R}^{2 n}$. Este es el espacio simpléctico para los campos vectoriales que utilizan posiciones y velocidades $(x, \dot{x}) \in T \mathbb{R}^{n}$.

Definición 1.9. El fibrado cotangente o haz cotangente de un espacio vectorial $W$ es el conjunto definido por

$$
T^{*} W=\left\{(q, p) ; q \in W, p \in W^{*}\right\},
$$

donde $p$ es una 1-forma diferencial en $W^{*}$.

$T^{*} W$ tiene una estructura simpléctica natural que podemos ver de la siguiente manera: consideremos dos puntos $x_{1}, x_{2} \in T^{*} W$ con coordenadas $x_{1}=\left(q_{1}, p_{1}\right)$ y $x_{2}=\left(q_{2}, p_{2}\right)$, donde $q_{i} \in W$ y $p_{i} \in W^{*}$.

Definimos la forma simpléctica natural del fibrado cotangente por

$$
\omega\left(x_{1}, x_{2}\right)=p_{2}\left(q_{1}\right)-p_{1}\left(q_{2}\right) \in \mathbb{R} .
$$

De manera directa podemos ver que $\omega$ es una forma bilineal, y antisimétrica. Además, es fácil verificar que es no degenerada.

DeFinición 1.10. Sean $(E, \omega)$ y $\left(E^{\prime}, \omega^{\prime}\right)$ dos espacios vectoriales simplécticos. Un simplectomorfismo $\varphi: E \rightarrow E^{\prime}$ es un isomorfismo lineal tal que preserva la estructura simpléctica entre los espacios, es decir

$$
\varphi^{*} \omega^{\prime}=\omega,
$$

donde $\varphi^{*} \omega^{\prime}(u, v)=\omega^{\prime}(\varphi(u), \varphi(v))$. Si existe un simplectomorfismo entre dos espacios vectoriales, se dice que son simplectomorfos.

\subsection{VARIEDADES SIMPLÉCTICAS.}

DEFINICIÓN 1.11. Una 2-forma diferencial $\omega$ definida sobre una variedad diferencial $M$ se dice que es de de Rham si para cada $x \in M$, la aplicación

$$
\omega_{x}: T_{x} M \times T_{x} M \rightarrow \mathbb{R},
$$

es bilineal y antisimétrica sobre el espacio tangente a $M$ en $x, y \omega_{x}$ varía de manera lisa en $x$. Se dice que $\omega$ es cerrada si satisface la ecuación diferencial $d \omega=0$, donde $d$ es la derivada exterior o diferencial de de Rham.

DeFinición 1.12. Una 2-forma $\omega$ definida sobre la variedad $M$, se dice simpléctica si es cerrada y si $\omega_{x}$ es simpléctica sobre $T_{x} M$ para todo $x \in M$.

Una estructura simpléctica sobre una variedad diferencial $M$ es una 2forma diferencial $\omega$ que es una forma simpléctica sobre $M$.

DeFinición 1.13. Una variedad simpléctica es una pareja $(M, \omega)$ donde $M$ es una variedad diferencial y $\omega$ es una forma simpléctica.

Es importante notar que una variedad simpléctica tiene dimensión par, ya que una forma antisimétrica sobre un espacio de dimensión impar es degenerada. 
Teorema 1.1 (Darboux). Sea $(M, \omega)$ una variedad simpléctica de dimensión $2 n$ y sea $x \in M$ un punto cualquiera sobre la variedad. Entonces existe una carta coordenada $\left(U, q_{1}, \cdots, q_{n}, p_{1}, \cdots, p_{n}\right)$ centrada en el punto $x$ tal que sobre $U$ se tiene

$$
\omega=\sum d p_{i} \wedge d q_{i}
$$

Demostración. Consideremos una base simpléctica para $T_{x} M$ y construyamos las coordenadas $\left(q_{1}^{\prime}, \cdots, q_{n}^{\prime}, p_{1}^{\prime}, \cdots, p_{n}^{\prime}\right)$ centradas en $x$ y que son válidas para una vecindad $U^{\prime}$, así tenemos

$$
\omega_{x}=\left.\sum d p_{i}^{\prime} \wedge d q_{i}^{\prime}\right|_{x} .
$$

Existen dos formas simplécticas sobre $U^{\prime}$ : la que se obtiene por $\omega_{0}=$ $\omega$ y $\omega_{1}=\sum d p_{i}^{\prime} \wedge d q_{i}^{\prime}$. Entonces existen vecindades $U_{0}$ y $U_{1}$ de $x$, y un difeomorfismo $\varphi: U_{0} \rightarrow U_{1}$ tal que

$$
\varphi(x)=x, \quad \text { y } \quad \varphi^{*}\left(\sum d p_{i}^{\prime} \wedge d q_{i}^{\prime}\right)=\omega .
$$

Como $\varphi^{*}\left(\sum d p_{i}^{\prime} \wedge d q_{i}^{\prime}\right)=\sum d\left(p_{i}^{\prime} \circ \varphi\right) \wedge d\left(q_{i}^{\prime} \circ \varphi\right)$ solo falta definir las nuevas coordenadas como $q_{i}=q_{i}^{\prime} \circ \varphi$ y $p_{i}=p_{i}^{\prime} \circ \varphi$.

Definición 1.14. Una carta definida por $\left(U, q_{1}, \cdots, q_{n}, p_{1}, \cdots, p_{n}\right)$ se conoce como una carta de Darboux.

1.2.1. El fibrado cotangente a una variedad. De manera equivalente a los espacios vectoriales donde se tiene una estructura simpléctica natural para su fibrado cotangente, el fibrado cotangente a una variedad $M=T^{*} \mathcal{Q}$ tiene una estructura natural de variedad simpléctica, para $\mathcal{Q}$ una variedad diferenciable de dimensión $n$.

Definición 1.15. Sean $\mathcal{Q}_{1}$ y $\mathcal{Q}_{2}$ dos variedades de dimensión $n$ con fibrados cotangentes $M_{1}=T^{*} \mathcal{Q}_{1}$ y $M_{2}=T^{*} \mathcal{Q}_{2}$ y 1-formas $\alpha_{1}$ y $\alpha_{2}$. Suponga que se tiene un difeomorfismo $f: \mathcal{Q}_{1} \rightarrow \mathcal{Q}_{2}$. El levantamiento cotangent 3 de $f$ es el difeomorfismo natural

$$
F:=T^{*} f: M_{1} \rightarrow M_{2},
$$

entre los fibrados cotangentes.

Para ser precisos, tenemos que si $x_{1}=\left(q_{1}, \xi_{1}\right) \in M_{1}$ con $q_{1} \in \mathcal{Q}_{1}$ y $\xi_{1} \in T_{q_{1}}^{*} \mathcal{Q}_{1}$, entonces se define

$$
F\left(x_{1}\right)=x_{2},
$$

donde $x_{2}=\left(q_{2}, \xi_{2}\right) \in M_{2}$ con $q_{2}=f\left(q_{1}\right) \in \mathcal{Q}_{2}$ y $\left(d f_{q_{1}}\right)^{*} \xi_{2}=\xi_{1}$, y además se tiene que

$$
\left(d f_{q_{1}}\right)^{*}: T_{q_{2}}^{*} \mathcal{Q}_{2} \rightarrow T_{q_{1}}^{*} \mathcal{Q}_{1},
$$

de tal forma que la restricción de $\left.F\right|_{T_{q_{1}}^{*}}$ es la aplicación inversa de $\left(d f_{q_{1}}\right)^{*}$.

\footnotetext{
${ }^{3} \mathrm{El}$ autor utiliza este término como traducción del original: cotangent lift
} 
Proposición 1.4. El levantamiento cotangente $F=T^{*} f$ de un difeomorfismo $f: \mathcal{Q}_{1} \rightarrow \mathcal{Q}_{2}$ lleva una 1-forma de Liouville sobre $T^{*} \mathcal{Q}_{2}$ en una 1-forma canónica o de Liouville sobre $T^{*} \mathcal{Q}_{1}$, es decir

$$
(F)^{*} \alpha_{2}=\alpha_{1} \text {. }
$$

Se debe notar que el diagrama

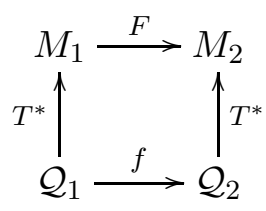

conmuta.

Corolario 1. El levantamiento $F$ de un difeomorfismo $f: \mathcal{Q}_{1} \rightarrow \mathcal{Q}_{2}$ es un simplectomorfismo, es decir

$$
(F)^{*} \omega_{2}=\omega_{1}
$$

donde $\omega_{1}$ y $\omega_{2}$ son las formas simplécticas canónicas sobre los fibrados cotangentes.

En resumen, un difeomorfismo de variedades induce un simplectomorfismo canónico entre los fibrados cotangentes:

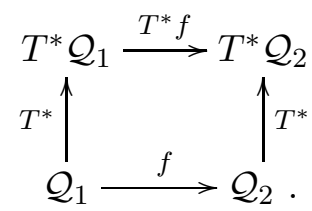

En términos del grupo de difeomorfismos $\operatorname{Dif}(N)$ y del grupo $S p(M, \omega)$ de simplectomorfismos, decimos que la aplicación

$$
\begin{aligned}
T^{*}: \operatorname{Dif}(N) & \rightarrow \operatorname{Sp}(M, \omega) \\
f & \mapsto F=T^{*} f,
\end{aligned}
$$

es un homomorfismo de grupos.

Definición 1.16. sea $X \in \mathfrak{X}(M)$ un campo vectorial sobre la variedad simpléctica $M$ y $\omega \in \Omega^{2}(M)$ una 2-forma diferenciable, el producto interno de $X$ y $\omega$, denotado por $i_{(X)} \omega$, está definido por

$$
i_{(X)} \omega(Y)=\omega(X, Y), \quad \text { para } Y \in \mathfrak{X}(M) .
$$

Si $\omega$ es no degenerada, esta aplicación genera un isomorfismo

$$
\mathfrak{X}(M) \underset{\omega^{\sharp}}{\stackrel{\omega^{b}}{\rightleftarrows}} \Omega^{1}(M) .
$$


En coordenadas simplécticas canónicas $x=(q, p)$ sobre $M$ tenemos entonces que $\omega=\sum_{j=1}^{n} d p_{j} \wedge d q_{j}$ y por lo tanto $\vartheta=i_{X} \omega$ es una 1 -forma

$$
\vartheta=\sum_{j=1}^{n}\left(a_{j}^{\prime} d q_{j}+a_{j}^{\prime \prime} d p_{j}\right) \in \Omega^{1}(M),
$$

el campo vectorial $\omega^{\sharp}(\vartheta)=X \in \mathfrak{X}(M)$ corresponde a

$$
X=\sum_{j=1}^{n}\left(a_{j}^{\prime \prime} \frac{\partial}{\partial q_{j}}-a_{j}^{\prime} \frac{\partial}{\partial p_{j}}\right) \in \mathfrak{X}(M) .
$$

1.2.2. Transformaciones SimplÉCtiCAS. Veamos ahora como se realiza la equivalencia entre dos variedades que posean una estructura simpléctica. De hecho, bajo el Teorema de Darboux se tiene que para cada $n \in \mathbb{N}$, todas las variedades simplécticas de dimensión $2 n$, son localmente difeomorfas al haz cotangente $M^{2 n} \cong T^{*} \mathbb{R}^{n}$.

Sea $\left(M_{1}, \omega_{1}\right)$ y $\left(M_{2}, \omega_{2}\right)$ dos variedades simplécticas de dimensión $2 n$.

DeFinición 1.17. Un difeomorfismo simpléctico o simplectomorfismo entre las variedades $\left(M_{1}, \omega_{1}\right)$ y $\left(M_{2}, \omega_{2}\right)$ es un difeomorfismo

$$
\varphi: M_{1} \rightarrow M_{2}
$$

tal que bajo $\varphi$ se preserva la estructura simpléctica, es decir que el "pullback" cumple

$$
\varphi^{*} \omega_{2}=\omega_{1} .
$$

Por definición si $u, v \in T_{p} M_{2}$ se tiene que

$$
\left(\varphi^{*} \omega_{2}\right)_{p}(u, v)=\left(\omega_{2}\right)_{\varphi(p)}\left(d \varphi_{p}(u), d \varphi_{p}(v)\right) .
$$

Se puede clasificar las variedades simplécticas módulo simplectomorfismos. El teorema de Darboux considera esta clasificación de manera local: la dimensión es el único invariante local de una variedad simpléctica módulo simplectomorfismos. De la misma forma en que una variedad diferencial de dimensión $n$ se ve localmente como $\mathbb{R}^{n}$, cualquier variedad simpléctica de dimensión $2 n$ se ve localmente como $\left(T^{*} \mathbb{R}^{n}, \omega_{0}\right)$.

Definición 1.18. Dos variedades $\left(M_{1}, \omega_{1}\right)$ y $\left(M_{2}, \omega_{2}\right)$ se dicen simplectomorfas si existe un difeomorfismo $\varphi: M_{1} \rightarrow M_{2}$ tal que $\varphi^{*} \omega_{2}=\omega_{1}$.

Definición 1.19. $\left(M_{1}, \omega_{1}\right)$ y $\left(M_{2}, \omega_{2}\right)$ se dicen isotópicas si existe una isotopía $\rho_{t}: M_{1} \rightarrow M_{2}$ tal que $\rho_{1}^{*} \omega_{2}=\omega_{1}$ y una familia suave $\omega_{t}:=\rho_{t}^{*} \omega_{2}$ de formas simplécticas que unen a $\omega_{1}$ y $\omega_{2}$ que son independientes de $t$.

Cuando se trabajan con la familia de simplectomorfismos sobre una misma variedad se tiene que $\varphi:(M, \omega) \rightarrow(M, \omega)$ debe preservar la estructura simpléctica, y por lo tanto

$$
\varphi^{*} \omega=\omega
$$


Podemos ver que si $\varphi_{1}$ y $\varphi_{2}$ son dos simplectomorfismos entonces $\varphi_{1} \circ \varphi_{2}$ cumple (véase la sección B.2)

$$
\left(\varphi_{1} \circ \varphi_{2}\right)^{*} \omega=\omega
$$

DEFINICIÓN 1.20. La familia de simplectomorfismos forman un grupo conocido como el grupo simpléctico de la variedad $M$ y se designa por $S p(M)$.

Por definición se puede considerar que

$$
\omega_{x}(u, v)=\omega_{\varphi(x)}(\varphi(u), \varphi(v)), \quad u, v \in T_{x} M .
$$

y entonces podemos identificar al grupo simpléctico, con el grupo de isometrías sobre $T_{x} M$ bajo la forma $\omega_{x}$ para todo $x \in M$.

1.2.3. TRANSFORMACIONES SIMPLÉCTICAS CON MULTIPLICADOR. Existe otro tipo de transformaciones que preserva la estructura simpléctica de una variedad, pero a diferencia de las transformaciones simplécticas canónicas, el elemento de área (el volumen en general) de la variedad se transforma mediante un multiplicador constante $\mu \in \mathbb{R}$ (o $\mathbb{C}$, y más generalmente $\mathbb{K}$ ).

DeFinición 1.21. Un transformación $\phi:(M, \omega) \rightarrow(M, \omega)$ que pertenece a los difeomorfismos de la variedad $\operatorname{Dif}(M)$ se dice que es simpléctica con multiplicador $\mu$ si se cumple

$$
\phi^{*} \omega=\frac{1}{\mu} \omega
$$

para $\mu \neq 0$.

Este tipo de transformaciones se utilizan para normalizar la atracción gravitacional, las unidades de masa o la velocidad angular de los sistemas de $N$ cuerpos de la mecánica celeste y trabajar con sistemas adimensionales preferentemente. En los casos en los que los sistemas Hamiltonianos son autónomos, al realizar una transformación simpléctica con multiplicador, la función Hamiltoniana solo se multiplicará por el factor $\mu$ (como se verá más adelante).

Proposición 1.5. Sea $\varphi:(M, \omega) \rightarrow(M, \omega)$ una transformación simpléctica con multiplicador $\mu \neq 0$ sobre una variedad de dimensión $2 n$. Consideremos que $M$ está parametrizada en coordenadas simplécticas $x=(q, p)$ con $q, p \in \mathbb{R}^{n}$. Entonces se cumple

$$
J=\mu\left(\frac{\partial \varphi}{\partial x}\right)^{t} J\left(\frac{\partial \varphi}{\partial x}\right)
$$

donde $\left(\frac{\partial \varphi}{\partial x}\right)$ es la matriz Jacobiana de la transformación. 
1.2.4. Subvariedades. En esta sección solo vamos a considerar subvariedades que están sumergidas de manera apropiada en la variedad simpléctica y de la cual heredan su topología.

Definición 1.22. Sea $(M, \omega)$ una variedad simpléctica de dimensión 2n. Una subvariedad $W \subset M$ es una subvariedad simpléctica (isotrópica $o$ coisotrópica) si, en cada punto $x \in W$ se tiene que $T_{x} W$ es un subespacio simpléctico (isotrópico o coisotrópico respectivamente) de $T_{x} M$.

Nosotros estaremos interesados particularmente en las subvariedades Lagrangianas que se encuentran en los sistemas completamente integrables y en las subvariedades coisotrópicas de codimensión 1 que corresponden a los niveles de energía de un sistema Hamiltoniano.

DeFinición 1.23. Sea $(M, \omega)$ una variedad simpléctica de dimensión $2 n$. Una subvariedad $\mathcal{L} \subset M$ se dice Lagrangiana si, en cada punto $x \in \mathcal{L}$ se tiene que $T_{x} \mathcal{L}$ es un subespacio Lagrangiano de $T_{x} M$, es decir, $\left.\omega_{x}\right|_{T_{x} \mathcal{L}} \equiv 0$ $y \operatorname{dim} T_{x} \mathcal{L}=\frac{1}{2} \operatorname{dim} T_{x} M$.

De manera equivalente, si se tiene la aplicación inclusión

$$
\imath: \mathcal{L} \hookrightarrow M,
$$

entonces $\mathcal{L}$ es una subvariedad Lagrangiana si y solo $\operatorname{si} \imath^{*} \omega=0$ y $\operatorname{dim} \mathcal{L}=$ $\frac{1}{2} \operatorname{dim} M$.

Si consideramos una variedad $\mathcal{Q}$ de dimensión $n$ con $M=T^{*} \mathcal{Q}$ su fibrado cotangente y designamos por $\left(q_{1}, \cdots, q_{n}\right) \in U \subset \mathcal{Q}$ sus coordenadas con las coordenadas cotangentes $\left(q_{1}, \cdots, q_{n}, p_{1}, \cdots, p_{n}\right) \in T^{*} U \subset M$, entonces la 1-forma de Liouville sobre $M$ es

$$
\alpha=\sum_{i} p_{i} d q_{i}
$$

y la 2-forma canónicat es

$$
\omega=d \alpha=\sum_{i} d p_{i} \wedge d q_{i}
$$

Definición 1.24. Se define la sección cero de un fibrado cotangente $M=T^{*} \mathcal{Q}$ como el conjunto de puntos

$$
\mathcal{Q}_{0}:=\left\{(q, \xi) \in T^{*} \mathcal{Q} \mid \xi=0, \xi \in T_{q}^{*} \mathcal{Q}\right\} .
$$

La sección cero forma una subvariedad $\mathcal{Q}_{0} \subset T^{*} \mathcal{Q}$ de dimensión $n$ y cuya intersección con $T^{*} U$ de un abierto $U \subset \mathcal{Q}$ está dada por las ecuaciones $\xi_{1}=\cdots=\xi_{n}=0$. Evidentemente se tiene que la 1-forma de Liouville

$$
\alpha=\sum_{i} \xi_{i} d q_{i} \equiv 0
$$

\footnotetext{
${ }^{4}$ Algunos autores consideran la forma simpléctica canónica como $\omega=-d \alpha=\sum_{i} d q_{i} \wedge$ $d p_{i}$
} 
se cancela sobre $\mathcal{Q}_{0} \cap T^{*} U$. En particular, si $\iota_{0}: \mathcal{Q}_{0} \hookrightarrow T^{*} \mathcal{Q}$ es la aplicación inclusión, tenemos que $\imath_{0}^{*} \alpha=0$. Consecuentemente $\imath_{0}^{*} \omega=\imath_{0}^{*} d \alpha=0$ y por lo tanto la sección cero $\mathcal{Q}_{0}$ es una subvariedad Lagrangiana.

Se puede construir toda una familia de subvariedades Lagrangianas que sean secciones caracterizadas por

$$
\mathcal{Q}_{\beta}=\left\{\left(q, \beta_{q}\right) \mid q \in \mathcal{Q}, \beta_{q} \in T_{q}^{*} \mathcal{Q}\right\},
$$

donde el covector $\beta_{q}$ depende de manera suave de $q$ y $\beta: \mathcal{Q} \rightarrow T^{*} \mathcal{Q}$ es una 1 -forma de de Rham. No es difícil probar que existe una correspondencia biunívoca entre el conjunto de subvariedades Lagrangianas de $T^{*} \mathcal{Q}$ definidas como secciones $\mathcal{Q}_{\beta}$ y el conjunto de 1-formas sobre $\mathcal{Q}$.

Definición 1.25. Sea $(M, \omega)$ una variedad simpléctica de dimensión $2 n$, es decir, $\omega$ es una forma bilineal, antisimétrica cerrada $(d \omega=0)$. Un campo vectorial $X$ sobre $M$ se dice que es campo vectorial de Liouville si se cumple $L_{X} \omega=\omega$, donde $L_{X}$ denota la derivada de Lie de la forma simpléctica a lo largo del campo vectorial $X$ (ver Apéndice A).

Con la ayuda de la fórmula de Cartan

$$
L_{X}=d \circ i_{X}+i_{X} \circ d,
$$

se puede reescribir la condición para un campo vectorial de Liouville como $d\left(i_{X} \omega\right)=\omega$. Entonces la 1-forma $\alpha=i_{X} \omega$ define una forma de contacto sobre cualquier hipersuperficie $\Sigma \subset M$ transversal al campo $X$. De hecho

$$
\alpha \wedge(d \alpha)^{n-1}=i_{X} \omega \wedge\left(d\left(i_{X} \omega\right)\right)^{n-1}=i_{X} \omega \wedge \omega^{n-1}=\frac{1}{n} i_{X}\left(\omega^{n}\right)
$$

es una forma de volumen sobre $\Sigma \subset M$ indicando que el campo $X$ es transversal a la hipersuperficie $\Sigma$.

Proposición 1.6. Toda superficie de nivel correspondiente a un valor regular de una función Hamiltoniana $H$ sobre la variedad simpléctica $(M, \omega)$ es una variedad de contacto.

1.2.5. REDUCCIÓN SIMPLÉCTICA. En esta sección enunciaremos un resultado que nos permite analizar los detalles más interesantes de un campo vectorial (Hamiltoniano) sobre una variedad simpléctica considerando el cociente de la variedad $M$ entre algún grupo de simetrías $G$. Para tal efecto, se requiere que $G$ sea un grupo de Lie y que actúe de manera Hamiltoniana sobre la variedad $M$, esto generará una foliación de la variedad $M$ por subvariedades determinadas por $G$.

Los resultados siguientes están escritos en términos de geometría simpléctica en una forma abstracta como se describe en [6. Sin embargo, utilizaremos la reducción simpléctica para la reducción de sistemas Hamiltonianos en la Sección [1.5] de una forma más concreta como se detalla en 83 . 
Proposición 1.7. Consideremos un espacio vectorial simpléctico $(E, \omega)$. Sea $\mathcal{L} \subset E$ un subespacio Lagrangiano y $W \subset E$ un subespacio coisotrópico de $E$ de tal forma que $W+\mathcal{L}=E$. Consideremos el espacio vectorial cociente $N=W / W^{\omega}$. Entonces

- $N$ es un subespacio vectorial simpléctico,

- $\left.\omega\right|_{N}$ es inyectiva,

- la imagen de la proyección de $\mathcal{L} \cap W$ en $N$ es un subespacio Lagrangiano.

Demostración. Primero veremos que la forma simpléctica induce una forma no degenerada sobre $N$, ya que $W^{\omega}$ es el kernel de la restricción de $\left.\omega\right|_{W}$ al subespacio $W$.

Al ser $W$ un subespacio coisotrópico entonces $W^{\omega} \subset W$ y por lo tanto $\mathcal{L} \cap\left(W \cap W^{\omega}\right)=\mathcal{L} \cap W^{\omega}$. Ahora, $\mathcal{L}=\mathcal{L}^{\omega}$ pues es un subespacio Lagrangiano y tendríamos sucesivamente

$$
\mathcal{L} \cap W^{\omega}=\left(\mathcal{L}^{\omega}+W\right)^{\omega}=(\mathcal{L}+W)^{\omega}=E^{\omega}=0 .
$$

Entonces el kernel de la proyección

$$
\mathcal{L} \cap W \rightarrow N,
$$

es trivial y la proyección es inyectiva. De manera inmediata tenemos que la restricción $\tilde{\omega}=\left.\omega\right|_{N}$ es no degenerada y por lo tanto $(N, \tilde{\omega})$ es un espacio simpléctico y por lo tanto un subespacio simpléctico de $E$.

Ahora debemos probar que $\mathcal{L} \cap W$ es un subespacio Lagrangiano de $(N, \tilde{\omega})$. Como $W$ es coisotrópico y $\mathcal{L}+W=E$ entonces $\mathcal{L} \cap W$ es un subespacio isotrópico de $E$, y tiene dimensión

$$
\operatorname{dim} \mathcal{L} \cap W=\operatorname{dim} \mathcal{L}+\operatorname{dim} W-\operatorname{dim}(\mathcal{L}+W)=\operatorname{dim} W-n,
$$

además la dimensión de $N$ es

$$
\operatorname{dim} N=\operatorname{dim} W-(2 n-\operatorname{dim} W)=2(\operatorname{dim} W-n) .
$$

Entonces $\mathcal{L} \cap W \subset N$ es un subespacio que tiene la mitad de la dimensión del superespacio. También debemos ver que $\mathcal{L} \cap W \subset \mathcal{L}$ y $\left.\omega\right|_{\mathcal{L} \cap W}=0$ por ser lagrangiano. De manera directa tenemos que $\left.\tilde{\omega}\right|_{\mathcal{L} \cap W}=0$, lo que indica que es un subespacio Lagrangiano.

\subsection{Sistemas Hamiltonianos.}

1.3.1. Campos vectoriales simplécticos y Hamiltonianos. Las principales familias de campos vectoriales que se encuentran sobre las variedades simplécticas son los campos vectoriales simplécticos y Hamiltonianos. En el primer caso, se encuentran estos campos como el álgebra de Lie del grupo de simplectomorfismos $S p(M)$, es decir el espacio de campos vectoriales cuyo flujo preserva la forma simpléctica. En el segundo caso, se considera 
aquellos campos vectoriales que corresponden a las 1-formas exactas sobre $M$ bajo el isomorfismo

$$
\omega^{\sharp}:=\left(i_{X} \omega\right)^{*}: \Omega^{1}(M) \rightarrow \mathfrak{X}(M) .
$$

Definición 1.26. Sea $(M, \omega)$ una variedad simpléctica. Un campo vectorial $X$ sobre $M$ se dice que es un campo vectorial simpléctico si

$$
L_{X} \omega=0,
$$

es decir, que la forma simpléctica es invariante bajo el flujo del campo vectorial.

El conjunto de campos vectoriales simplécticos sobre $M$ se denotará por $\mathfrak{s p}(M)$. Es fácil ver que los campos vectoriales simplécticos forman un álgebra de Lie, para ello seleccionemos dos campos arbitrarios $X, Y \in \mathfrak{s p}(M)$ y veamos que

$$
L_{[X, Y]} \omega=\left[L_{X}, L_{Y}\right] \omega=L_{X}\left(L_{Y} \omega\right)-L_{Y}\left(L_{X} \omega\right)=0 .
$$

Adicionalmente, los campos vectoriales simplécticos se encuentran como el álgebra de Lie del grupo de simpléctomorfismos sobre la variedad.

Ahora utilicemos la identidad de Cartan para determinar quienes son los campos Hamiltonianos, como subconjunto de los campos simplécticos

$$
L_{X} \omega=d i_{X} \omega+i_{X} d \omega=0,
$$

y como se tiene que $d \omega=0$ se tiene realmente que $L_{X} \omega=d i_{X} \omega$ así que los campos simplécticos son aquellos donde $i_{X} \omega$ es una 1-forma cerrada. Ahora se debe caracterizar a aquellos campos que cumplen que la 1-forma $i_{X} \omega$ sea exacta.

DEFINICIÓN 1.27. Dada una función $H \in \mathfrak{F}(M)$ sobre la variedad simpléctica $(M, \omega)$, el campo vectorial

$$
X_{H}=\omega^{\sharp}(d H),
$$

es llamado un campo vectorial Hamiltoniano asociado a $\mathrm{H}$. El conjunto de los campos vectoriales Hamiltonianos se denotará por $\mathfrak{h a m}(M) \subset \mathfrak{s p}(M)$.

Se puede mostrar que si consideramos $X, Y \in \mathfrak{s p}(M)$ y $Z \in \mathfrak{X}(M)$ bajo el producto interno $i_{[X, Y]} \omega(Z):=w([X, Y], Z)$ se obtiene

$$
i_{[X, Y]} \omega=d(\omega(Y, X))
$$

(88, pag 13). Es decir, que la inclusión del campo $[X, Y]$ con la forma simpléctica $\omega$ equivale a considerar la diferencial de una función $f: M \rightarrow \mathbb{R}$, lo que indica que la forma $\omega$ es exacta y por lo tanto el campo $[X, Y]$ es un campo Hamiltoniano es decir que

$$
[\mathfrak{s p}(M), \mathfrak{s p}(M)] \subset \mathfrak{h a m}(M) .
$$

Como $\mathfrak{s p}(M)$ es un álgebra de Lie entonces $\mathfrak{h a m}(M)$ hereda la estructura de álgebra de Lie y adicionalmente es un ideal de $\mathfrak{s} \mathfrak{p}(M)$. 
Finalmente, podemos considerar el cociente $\mathfrak{s p}(M) / \mathfrak{h a m}(M):=H_{d R}^{1}(M)$ que tendrá una estructura inducida de álgebra de Lie que generará la sucesión exacta

$$
0 \rightarrow \mathfrak{h a m}(M) \hookrightarrow \mathfrak{s p}(M) \rightarrow H_{d R}^{1}(M) \rightarrow 0 .
$$

$H_{d R}^{1}(M)$ se conoce como el primer grupo de Homologías de de Rham de $M$ y en este caso se considera como un álgebra de Lie conmutativa.

Definición 1.28. Sea $(M, \omega)$ una variedad simpléctica y sea $H \in \mathfrak{F}(M)$ una función sobre $M$ con campo vectorial Hamiltoniano $X_{H}$. A la terna $\left(M, \omega, X_{H}\right)$ se le llama un sistema Hamiltoniano.

Se debe notar que las condiciones

- $i_{\left(X_{H}\right)} \omega=d H$

- $X_{H}=\omega^{\sharp}(d H)$,

son equivalentes.

Para verificar las ecuaciones del campo vectorial en coordenadas locales, se debe determinar un conjunto de cartas $(U, \phi)$ para la variedad $M$ de tal forma que $\phi: U \rightarrow V$ con $V \subset \mathbb{R}^{2 n}$ con regla de correspondencia $\phi(\mathbf{q})=\mathbf{x}$ para $\mathbf{q}=\left(q_{1}, \ldots, q_{n}, p_{1}, \ldots, p_{n}\right) \in M$ y $\mathbf{x}=\left(x_{1}, \ldots, x_{n}, y_{1}, \ldots, y_{n}\right) \in \mathbb{R}^{2 n}$. Por lo tanto, como $i_{\left(X_{H}\right)} \omega=d H$ entonces obtenemos

$$
\dot{\mathbf{q}}=J \nabla H(\mathbf{q}) \text {. }
$$

Si consideramos $\mathbf{q}=(q, p)$ el sistema se puede escribir de la forma

$$
\begin{aligned}
\dot{q}_{i} & =\frac{\partial H}{\partial p_{i}} \\
\dot{p}_{i} & =-\frac{\partial H}{\partial q_{i}}
\end{aligned}
$$

para $i=1,2, \ldots, n$. Estas son las ecuaciones de Hamilton.

En la teoría de ecuaciones diferenciales son utiles los siguientes conceptos

DeFinición 1.29. Si un sistema Hamiltoniano está definido sobre una variedad simpléctica de dimensión $2 n$, entonces al valor $n$ se le conoce como el número de grados de libertad del sistema.

Definición 1.30. Decimos que un sistema Hamiltoniano (1.5) es autónomo si $H$ no depende explícitamente de $t$. En este caso $H$ es una constante de movimiento y se dice que es una integral primera.

1.3.2. Sistemas Completamente integrables. La relación (1.4) se puede esribir de manera alternativa como

$$
[X, Y]=X_{w(Y, X)},
$$

para $X, Y \in \mathfrak{s p}(M)$. Si consideramos funciones $f, g \in \mathfrak{F}(M)$ y sus correspondientes campos vectoriales simplécticos $X_{f}, X_{g}$, podemos escribir

$$
\left[X_{f}, X_{g}\right]=X_{w\left(X_{g}, X_{f}\right)}
$$


que es un campo vectorial Hamiltoniano. Esta campo esta definido por dos funciones suaves $f, g$ sobre la variedad simpléctica $M$, entonces tenemos la siguiente

Definición 1.31. Definimos el corchete de Poisson de dos funciones $f, g \in \mathfrak{F}(M)$ como la función

$$
\{f, g\}=w\left(X_{f}, X_{g}\right) .
$$

Proposición 1.8. Sean $f, g \in \mathfrak{F}(M)$ funciones suaves sobre la variedad $M$ entonces se cumple

1. $\{f, g\}=X_{f}(g)$,

2. $\left[X_{f}, X_{g}\right]=X_{\{g, f\}}$.

Demostración. 1. Aplicamos directamente la definición

$$
\begin{aligned}
\{f, g\} & =\left(i_{X_{g}} \omega\right)\left(X_{f}\right), \\
& =(d g)\left(X_{f}\right), \\
& =X_{f} g .
\end{aligned}
$$

2. De la expresión (1.4) tenemos

$$
\begin{aligned}
{\left[X_{f}, X_{g}\right] } & =X_{w\left(X_{g}, X_{f}\right)} \\
& =X_{\{g, f\}} .
\end{aligned}
$$

Teorema 1.2. El corchete de Poisson es una estructura de álgebra de Lie sobre $\mathfrak{F}(M)$ y la aplicación

$$
\begin{aligned}
j: \mathfrak{F}(M) & \rightarrow \mathfrak{h a m}(M, \omega), \\
f & \mapsto X_{f} .
\end{aligned}
$$

es un homomorfismo de álgebras de Lie.

El kernel del homomorfismo $j$ corresponde a todas las funciones tales que $d f=0$. Si $M$ es conexo entonces el kernel corresponde a todas las funciones constantes y se puede identificar una copia de $\mathbb{R} \subset \mathfrak{F}(M)$ como el subconjunto de funciones constantes sobre $M$. De aquí se puede obtener otra sucesión exacta

$$
0 \rightarrow \mathbb{R} \stackrel{\imath}{\hookrightarrow} \mathfrak{F}(M) \stackrel{j}{\rightarrow} \mathfrak{h a m}(M, \omega) \rightarrow 0 .
$$

Definición 1.32. Consideremos una variedad simpléctica $(M, \omega)$ de dimensión $2 n$ y un conjunto de $m \leq n$ funciones $F_{1}, \cdots, F_{m} \in \mathfrak{F}(M)$. Decimos que las funciones $\left\{F_{i}\right\}_{i=1}^{m}$ están en involución si se cumple

$$
\left\{F_{i}, F_{j}\right\}=0, \quad i, j=1, \cdots, m,
$$

y sus diferenciales son linealmente independientes punto a punto en un conjunto abierto $U \subset M$. 
Definición 1.33. Sea $\left(M, \omega, X_{H}\right)$ un sistema Hamiltoniano y $F \in \mathfrak{F}(M)$ una función lisa sobre la variedad $M . F$ se dice que es una integral primera si está en involución con la función Hamiltoniana $H$.

Definición 1.34. Un sistema Hamiltoniano $\left(M, \omega, X_{H}\right)$ con $n$ grados de libertad 5 se dice que es completamente integrable si existen $n$ funciones $F_{1}, \cdots, F_{n}$ que están en involución para toda $i=1, \cdots, n$ y se tiene $F_{1}=H$.

Nótese que la condición de independencia lineal sobre los puntos de un abierto de $U \subset M$ se ha considerado dentro de la definición de involución. Esto se debe a que deseamos realizar diversos procesos como la reducción simpléctica de sistemas Hamiltonianos mediante foliaciones no degeneradas.

Teorema 1.3. Sea $\left(M, \omega, X_{H}\right)$ un sistema Hamiltoniano completamente integrable entonces el flujo del campo vectorial $X_{H}$ corresponde a lineas rectas sobre cilindros de la forma $\mathbb{R}^{k} \times \mathbb{T}^{n-k}$ para alguna $1 \leq k \leq n$.

1.3.3. Hamiltonianos no-Autónomos. A menudo es útil considerar funciones Hamiltonianas que dependen del tiempo

$$
H: M \times[0,1] \rightarrow \mathbb{R},
$$

que denotaremos por $H(p, t)=H_{t}(p)$. El campo vectorial se define de manera análoga al caso original $X_{H_{t}}$ y entonces se tiene una familia de difeomorfismos $\phi_{t}^{H}$, tal que $\phi_{0}^{H}=i d$ que al tiempo $t$ son tangentes a $X_{H_{t}}$, es decir

$$
\frac{d}{d t}\left(\phi_{t}^{H}(p)\right)=X_{H_{t}}\left(\phi_{t}^{H}(p)\right), \quad p \in M, t \in[0,1] .
$$

Dicha familia de difeomorfismos preserva la estructura simpléctica y de hecho forman una familia de simplectomorfismos llamada una isotopía Hamiltoniana.

\subsection{ACCiOnes Hamiltonianas y LA APLICACIÓN MOMENTO.}

Toda función suave $F \in \mathfrak{F}(M)$ definida sobre una variedad simpléctica con un sistema Hamiltoniano $\mathcal{H}=\left(M, \omega, X_{H}\right)$ genera una familia de simplectomorfismos y si además $\{F, H\}=0$, entonces los valores de la función $F$ se conservan a lo largo del campo vectorial $X_{H}$ y por lo tanto, los simplectomorfismos serán una familia de transformaciones simétricas. Un sistema Hamiltoniano $\mathcal{H}$ puede tener diversas cantidades conservadas $F_{i}$. Si la variedad simpléctica tiene dimensión $2 n$ y existen $n$ cantidades conservadas independientes funcionalmente $F_{i}$, con $H=F_{1}$, y que están en involución (i.e., $\left\{F_{i}, F_{j}\right\}=0$ ), entonces el sistema Hamiltoniano es integrable en el sentido de Liouville.

\footnotetext{
${ }^{5}$ Es decir, la variedad simpléctica $M$ tiene dimensión $2 n$.
} 
DeFINICIÓN 1.35. Una acción de un grupo de Lie G sobre una variedad $M$ es un homomorfismo de grupos

$$
\begin{aligned}
\varphi: G & \rightarrow \operatorname{Dif}(M), \\
g & \mapsto \varphi_{g},
\end{aligned}
$$

donde $\operatorname{Dif}(M)$ es el grupo de difeomorfismos sobre $M$.

Es conveniente pensar en el flujo $\varphi_{t}=\varphi(t)$ de un campo vectorial como la acción de un grupo de Lie sobre la variedad $M$. Entonces la evaluación de la acción del grupo será

$$
\begin{aligned}
\operatorname{eval}_{\varphi}: G \times M & \rightarrow M, \\
(g, p) & \mapsto \varphi_{g}(p) .
\end{aligned}
$$

Ahora consideremos una variedad simpléctica $(M, \omega)$ y un grupo de Lie $G$ con la acción (1.6).

Definición 1.36. Decimos que la acción $\varphi$ es una acción simpléctica si ésta actúa por simplectomorfismos,

$$
\varphi: G \rightarrow S p(M)
$$

es decir, para cada $g \in G$ se tiene que $\varphi_{g}^{*}(\omega)=\omega$.

Si además consideramos el álgebra de Lie $\mathfrak{g}=\operatorname{Lie}(G)$ y su espacio vectorial dual $\mathfrak{g}^{*}$, podemos definir una acción hamiltoniana de la siguiente manera

DEFINICIÓN 1.37. Decimos que una acción $\varphi$ es una acción hamiltoniana si existe una aplicación

$$
\mu: M \rightarrow \mathfrak{g}^{*},
$$

que satisface las condiciones siguientes:

- Para cada $X \in \mathfrak{g}$ caracterizamos a la componente de $\mu$ a lo largo de $X$ como

$$
\begin{aligned}
\mu^{X}: M & \rightarrow \mathbb{R} \\
p & \mapsto\langle\mu(p), X\rangle,
\end{aligned}
$$

y designamos $\hat{X}$ el campo vectorial sobre $M$ generado por el subgrupo de un parámetro $\{\exp (t X) \mid t \in \mathbb{R}\} \subseteq G$. Entonces

$$
d \mu^{X}=i_{\hat{X}} \omega
$$

es decir, la función $\mu^{X}$ es una función Hamiltoniana para el campo vectorial $\hat{X}$.

- La aplicación $\mu$ es equivariante con respecto de la acción $\varphi$ de $G$ sobre $M$ y la acción coadjunta Ad* de G sobre $\mathfrak{g}^{*}$ cumple

$$
\mu \circ \varphi_{g}=\operatorname{Ad}_{g}^{*} \circ \mu, \quad \forall g \in G,
$$


es decir, el siguiente diagrama conmuta

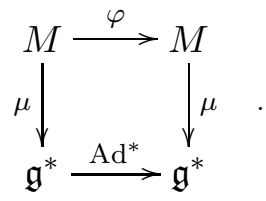

De esta forma $(M, \omega, G, \mu)$ es llamado un $G$-espacio hamiltoniano y $\mu$ es llamada la aplicación momento. A la configuración $(M, \omega, G, \mu)$ también se le conoce como un sistema Hamiltoniano con G-simetría, aunque este término es más conocido cuando la variedad tiene una estructura de Poisson.

TeOrema 1.4. Si la función Hamiltoniana $H: M \rightarrow \mathbb{R}$ de un sistema mecánico es invariante bajo la acción Hamiltoniana del grupo de Lie G sobre la variedad $M$, la aplicación momento correspondiente $\mu: M \rightarrow \mathfrak{g}^{*}$ es una primera integral del sistema.

Si restringimos esta situación al toro $\mathbb{T}^{k}=\mathbb{S}^{1} \times \cdots \times \mathbb{S}^{1}$ entonces el grupo de Lie $G=\mathbb{T}^{k}=\left(\mathbb{S}^{1}\right)^{k}$ que actúa sobre una variedad simpléctica $(M, \omega)$ tiene la característica de que tanto el álgebra de Lie $\mathfrak{g}$ como su dual $\mathfrak{g}^{*}$ se identifican con el espacio euclidiano $\mathbb{R}^{k}$. Como la acción coadjunta del toro es trivial entonces la aplicación momento $\mu: M \rightarrow \mathbb{R}^{k}$ sólo necesita satisfacer que para la proyección $\mu^{X}: M \rightarrow \mathbb{R}^{k}$ sobre cada campo $X \in \mathfrak{g} \cong \mathbb{R}^{k}$ se cumpla

$$
d \mu^{X}=i_{\hat{X}} \omega
$$

y que sea invariante bajo la acción del toro. Cada campo fundamental $X_{i}$ de un sistema Hamiltoniano integrable es generado por una integral primera $F_{i}, 1 \leq i \leq n$. La aplicación

$$
\mu=\left(H=F_{1}, \ldots, F_{k}\right): M \rightarrow \mathfrak{g}^{*} \cong \mathbb{R}^{k},
$$

es precisamente una aplicación momento como fue definido en la sección anterior. Si $k<n$ el sistema es parcialmente integrable, por otro lado si $k=n$ el sistema se dice Liouville integrable o completamente integrable.

También se debe notar que para una acción Hamiltoniana de un toro $\mathbb{T}^{k}$, la condición de equivariancia se convierte en invariancia.

Es un dato conocido que la imagen inversa $\mu^{-1}(x)$ de cada $x \in \operatorname{Im} g(\mu)$ es una subvariedad Lagrangiana de $(M, \omega)$. En el caso más general cuando la variedad simpléctica donde se define el sistema Hamiltoniano es no compacta, la imagen de la aplicación momento se puede separar en regiones, que determinan la topología de cada fibra $\mu^{-1}(x)$. Si la fibra define una variedad compacta, entonces será isomorfa a un toro. En otros casos, será isomorfa a cilindros o planos de acuerdo de la región donde se encuentre $x$. Más adelante se verá esta correspondencia en el problema circular doble de Sitnikov. 


\subsection{ReduCCión DE SISTEMAS HAMILTONIANOS CON SIMETRÍA.}

En esta sección retomamos el concepto de reducción simpléctica que ya se ha estudiado para espacios vectoriales lineales, y lo generalizaremos para ser aplicado a variedades simpléctias.

Supongamos que se tiene una acción Hamiltoniana de un grupo de Lie $G$ sobre una variedad simpléctica $(M, \omega)$ y consideremos la aplicación momento $\mu: M \rightarrow \mathfrak{g}^{*}$ correspondiente. Consideremos el conjunto de nivel de la aplicación, es decir, la imagen inversa de un punto regular $x \in \mathfrak{g}^{*}$ bajo $\mu$ y denotemos a este conjunto por $M_{x}=\mu^{-1}(x)$. Como $x$ es un valor regular de $\mu$ se tiene que la diferencial $d \mu$ en todo punto $c \in M_{x}$ envía

$$
T_{c} M \rightarrow \mathfrak{g}^{*},
$$

o en otro caso $M_{x}=\varnothing . M_{x}$ es una variedad suave y de hecho es una subvariedad coisotrópica de $M$. Si $\operatorname{dim} G=\frac{1}{2} \operatorname{dim} M$ entonces $M_{x}$ será una subvariedad Lagrangiana. En el último caso se cumple que la forma simpléctica de la variedad restringida al conjunto de nivel es $\left.\omega\right|_{M_{x}} \equiv 0$.

Definición 1.38. El subgrupo de isotropía 6 de $x$ relativo a la acción coadjunta del grupo de Lie $G$ está dado por

$$
G_{x}=\left\{g \in G \mid A d_{g}^{*} x=x\right\} .
$$

DeFinición 1.39. La órbita de un punto $m \in M$ bajo la acción del grupo $G$ es el conjunto

$$
G m=\left\{\phi_{g} m \mid g \in G\right\} .
$$

Lema 1.1. El subgrupo de isotropía de $x$ deja invariante a $M_{x}$.

Se asume que $G_{x}$ es un grupo compacto y que actúa sobre $M_{x}$ de manera efectiva y sin puntos fijos (en realidad basta con que la acción de $G_{x}$ sobre $M_{x}$ sea propia y no se requiere la compacidad del grupo). El espacio $M_{x}$ se descompone en órbitas de la acción de $G_{x}$ y bajo estas hipótesis el espacio de las órbitas $\mathcal{O}_{x}=M_{x} / G_{x}$ es una variedad suave llamada el espacio fase reducido.

Teorema 1.5. Sea $(M, \omega, G, \mu)$ un G-espacio Hamiltoniano con una aplicación momento equivariante $\mu$ que satisface las condiciones anteriores. Entonces $\mathcal{O}_{x}=\mu^{-1}(x) / G_{x}$ tiene una forma simpléctica $\omega_{x}$ definida de manera única con

$$
\pi_{x}^{*} \omega_{x}=i_{x}^{*} \omega
$$

donde $\pi_{x}: \mu^{-1}(x) \rightarrow \mathcal{O}_{x}$ es la proyección canónica $e i_{x}: \mu^{-1}(x) \hookrightarrow M$ es la inclusión.

La prueba del teorema requiere de un resultado previo

\footnotetext{
${ }^{6}$ A este subgrupo también se le conoce como subgrupo estabilizador.
} 
Lema 1.2. Sea $m \in \mu^{-1}(x)$, entonces las siguientes propiedades se cumplen

1. $G_{x} m \subset \mu^{-1}(x)$,

2. $T_{m} G_{x} m=T_{m}(G m) \cap T_{m}\left(\mu^{-1}(x)\right)$,

3. $T_{m}\left(\mu^{-1}(x)\right)$ y $T_{m}(G m)$ son espacios $\omega$-ortogonales.

Básicamente, este lema establece las relaciones entre los espacios tangentes a $m$ en las dimensiones adecuadas de forma que se pueda utilizar la Proposición 1.7 para reducción simpléctica en espacios vectoriales (lineales). Las pruebas de estos resultados pueden consultarse en 1, 9, 83, 87.

Con la forma $\omega_{x}, \mathcal{O}_{x}$ hereda una estructura simpléctica de forma natural y entonces la pareja $\left(\mathcal{O}_{x}, \omega_{x}\right)$ será una variedad simpléctica.

Sea $\left(M, \omega, X_{H}, G\right)$ un sistema Hamiltoniano con una $G$-simetría. Como sabemos, $X_{H}$ es $G$-invariante y tangente a $M_{x}=\mu^{-1}(x)$. Entonces se obtiene un campo vectorial $\tilde{X}_{H}=\left.X_{H}\right|_{\mathcal{O}_{x}}$ sobre $\mathcal{O}_{x}$ llamado el campo vectorial reducido.

TEOREMA 1.6. El campo vectorial reducido sobre el espacio fase reducido es Hamiltoniano. La función Hamiltoniana correspondiente $\tilde{H}$ coincide con la función Hamiltoniana original restringida a $\mathcal{O}_{x}$.

De hecho, la función Hamiltoniana original es invariante bajo la acción del grupo $G$, lo que implica que $H(m)=H(\mathrm{gm})$ para $m=(q, p) \in M \mathrm{y}$ para toda $g \in G$.

\subsection{FunCIONES GENERATRICES.}

Como ya vimos anteriormente, la condición para tener un simplectomorfismo entre dos variedades simplécticas $\varphi:\left(M_{1}, \omega_{1}\right) \rightarrow\left(M_{2}, \omega_{2}\right)$ es que el "pullback" de la forma simpléctica de la imagen de $\varphi$ sea la forma simpléctica del dominio, es decir

$$
\varphi^{*} \omega_{2}=\omega_{1}
$$

al menos localmente.

Uno de los métodos que se utilizan para definir simplectomorfismos es el que utiliza funciones generatrices y que aprovecha diversas propiedades de las variedades simplécticas y de sus simplectomorfismos. Necesitaremos los siguientes resultados:

Proposición 1.9. Sean $\left(M_{1}, \omega_{1}\right)$ y $\left(M_{2}, \omega_{2}\right)$ dos variedades simplécticas. Consideremos el producto cartesiano de las variedades $M=M_{1} \times M_{2}$ y las proyecciones canónicas $\pi_{i}: M_{1} \times M_{2} \rightarrow M_{i}, i=1,2$, sobre cada componente. Entonces la variedad $\left(M_{1} \times M_{2}, \Omega\right)$ con

$$
\Omega=\pi_{1}^{*} \omega_{1}-\pi_{2}^{*} \omega_{2},
$$

es una veriedad simpléctica. 
Proposición 1.10. La gráfica $\Gamma_{\rho}$ de un simplectomorfismo

$$
\rho: M_{1} \rightarrow M_{2}
$$

es una subvariedad Lagrangiana de $M=M_{1} \times M_{2}$.

Demostración. Probaremos que el "pullback" de la inclusión es idénticamente cero $i_{\rho}^{*} \Omega=0$ en $M$. Primero veamos que $\rho$ induce un difeomorfismo de $M_{1} \rightarrow \Gamma_{\rho}$, así que podemos escribir

$$
T_{(x, \rho(x))} \Gamma_{\rho}=\left\{(v, T \rho(v)) \mid v \in T_{x} M_{1}\right\} .
$$

Ahora, por la definición de $\Omega$ tenemos

$$
\begin{aligned}
i_{\rho}^{*} \Omega\left(\left(v_{1}, T \rho\left(v_{1}\right)\right),\left(v_{2}, T \rho\left(v_{2}\right)\right)\right) & =\omega_{1}\left(v_{1}, v_{2}\right)-\omega_{2}\left(T \rho\left(v_{1}\right), T \rho\left(v_{2}\right)\right), \\
& =\omega_{1}\left(v_{1}, v_{2}\right)-\rho^{*} \omega_{2}\left(v_{1}, v_{2}\right), \\
& =\left(\omega_{1}-\rho^{*} \omega_{2}\right)\left(v_{1}, v_{2}\right),
\end{aligned}
$$

y por lo tanto $i_{\rho}^{*} \Omega \equiv 0$ si y sólo si $\rho^{*} \omega_{2}=\omega_{1}$.

Ahora veamos como funciona el proceso para generar simplectomorfismos con funciones generadoras:

Primero se considera la variedad producto de dos variedades simplécticas de la misma dimensión $\left(M_{1} \times M_{2}, \Omega\right)$ con una forma simpléctica adecuada. A continuación se considera una subvariedad Lagrangiana $\mathcal{L} \subset\left(M_{1} \times M_{2}, \Omega\right)$ y se considera una involución $\sigma: M_{2} \rightarrow M_{2}$ que definiremos más adelante. Esta involución torcerá $\rrbracket^{7}$ a la subvariedad Lagrangiana y a la forma simpléctica por lo que tendremos la subvariedad torcida $\mathcal{L}^{\sigma} \subset\left(M_{1} \times M_{2}, \Omega^{\sigma}\right)$. Posteriormente verificamos si $\mathcal{L}^{\sigma}$ es la gráfica de un difeomorfismo $\rho: M_{1} \rightarrow M_{2}$ en caso afirmativo, entonces $\rho: M_{1} \rightarrow M_{2}$ es un simplectomorfismo.

Para toda $f \in \mathfrak{F}\left(N_{1} \times N_{2}\right)$, df es una 1-forma cerrada sobre $\left(N_{1} \times N_{2}\right)$. La subvariedad Lagrangiana generada por $f$ es

$$
\mathcal{L}_{f}:=\left\{\left((x, y),(d f)_{(x, y)}\right) \mid(x, y) \in N_{1} \times N_{2}\right\} .
$$

Adoptaremos la notación

$$
\begin{aligned}
d_{x} f:=\pi_{x}(d f)_{(x, y)}: T_{x} N_{1} \times T_{y} N_{2} \rightarrow T_{x} N_{1} \times\{0\}, \\
d_{y} f:=\pi_{y}(d f)_{(x, y)}: T_{x} N_{1} \times T_{y} N_{2} \rightarrow\{0\} \times T_{y} N_{2},
\end{aligned}
$$

que nos permite escribir

$$
\mathcal{L}_{f}=\left\{\left(x, y, d_{x} f, d_{y} f\right) \mid(x, y) \in N_{1} \times N_{2}\right\}
$$

$\mathrm{y}$

$$
\mathcal{L}_{f}^{\sigma}=\left\{\left(x, y, d_{x} f,-d_{y} f\right) \mid(x, y) \in N_{1} \times N_{2}\right\} .
$$

Cuando $\mathcal{L}_{f}^{\sigma}$ es de hecho la gráfica de un difeomorfismo $\phi: M_{1} \rightarrow M_{2}$, llamaremos a $\phi$ el simplectomorfismo generado por $f$, y llamaremos a $f$ la función generadora de $\phi: M_{1} \rightarrow M_{2}$.

\footnotetext{
${ }^{7}$ Traducción literal del autor para el término twist
} 
Pero ¿cuando sabremos que $\mathcal{L}_{f}^{\sigma}$ es la gráfica de un difeomorfismo? Sea $\left(U_{1}, x_{1}, \cdots, x_{n}\right),\left(U_{2}, y_{1}, \cdots, y_{n}\right)$ cartas coordenadas para $N_{1}$ y $N_{2}$, con las cartas asociadas $\left(T^{*} U_{1}, x_{1}, \cdots, x_{n}, \xi_{1}, \cdots, \xi_{n}\right),\left(T^{*} U_{2}, y_{1}, \cdots, y_{n}, \eta_{1}, \cdots, \eta_{n}\right)$ para $M_{1}$ y $M_{2}$. El conjunto

$$
\mathcal{L}_{f}^{\sigma}=\left\{\left(x, y, d_{x} f,-d_{y} f\right) \mid(x, y) \in N_{1} \times N_{2}\right\}
$$

es la gráfica de $\phi: M_{1} \rightarrow M_{2}$ si y sólo si, para cualquier $(x, \xi) \in M_{1}$ y $(y, \eta) \in M_{2}$ se tiene

$$
\phi(x, \xi)=(y, \eta)
$$

y esto se da si y sólo si

$$
\xi=d_{x} f \quad \text { y } \quad \eta=-d_{y} f .
$$

Por lo tanto, dado un punto $(x, \xi) \in M_{1}$, para encontrar su imagen $(y, \eta)=$ $\phi(x, \xi)$, debemos resolver las ecuaciones Hamiltonianas

$$
\begin{aligned}
\xi_{i} & =\frac{\partial f}{\partial x_{i}}(x, y), \\
\eta_{i} & =-\frac{\partial f}{\partial y_{i}}(x, y) .
\end{aligned}
$$

Si existe una solución $y=\phi_{1}(x, \xi)$, debemos revisar la otra ecuación para obtener $\eta=\phi_{2}(x, \xi)$, de tal forma que

$$
\phi(x, \xi)=\left(\phi_{1}(x, \xi), \phi_{2}(x, \xi)\right) .
$$

Ahora por el teorema de la función implícita, para resolver la primera ecuación de manera local para $y$ en términos de $x$ y $\xi$, necesitamos la condición

$$
\operatorname{det}\left(\frac{\partial}{\partial y_{j}}\left(\frac{\partial f}{\partial x_{i}}\right)\right)_{i, j=1}^{n} \neq 0 .
$$

Esta es una condición local necesaria y suficiente para que $f$ pueda generar un simplectomorfismo $\rho$. Sin embargo, globalmente se requiere biyectividad.

1.6.1. CoOrdenadas ACCión-Ángulo. Las coordenadas acción-ángulo, se utilizan en los sistemas Hamiltonianos completamente integrables cuando el álgebra de Lie $\mathfrak{g}$ asociada a la imagen de la aplicación momento $\mu$ es conmutativa. En este caso, cada campo vectorial fundamental $Y \in \operatorname{Img}(\mu)$ permite reconstruir localmente, a través de la aplicación exponencial, un campo vectorial Hamiltoniano en una subvariedad de dimensión 2. Esto permite considerar la acción Hamiltoniana de $\mathbb{S}^{1}$ o de $\mathbb{R}$ sobre la restricción del campo a $N=\left(q_{i}, p_{i}\right)$ para $1 \leq i \leq n$.

Si $(M, \omega)$ es una variedad simpléctica compacta de dimensión $2 n$, entonces existe una 1-forma tal que $\omega=d \lambda$. Para todo simplectomorfismo 
$\varphi \in S p(M)$, la 1-forma $\lambda-\varphi^{*} \lambda$ es cerrada ya que

$$
\begin{aligned}
d\left(\lambda-\varphi^{*} \lambda\right) & =\omega-d \varphi^{*} \lambda, \\
& =\omega-\varphi^{*} d \lambda, \\
& =\omega-\varphi^{*} \omega, \\
& =0 .
\end{aligned}
$$

Aplicando el Lemma de Poincaré (véase la sección B.2.5) sabemos que localmente existe $f: M \rightarrow \mathbb{R}$ tal que $d f=\lambda-\varphi^{*} \lambda$. Integremos sobre una curva simple cerrada $\gamma$ para obtener

$$
f=\int_{\gamma} \lambda=\int_{\gamma} \varphi^{*} \lambda
$$

y finalmente veremos que

$$
\int_{\gamma} \lambda=\int_{\varphi(\gamma)} \lambda
$$

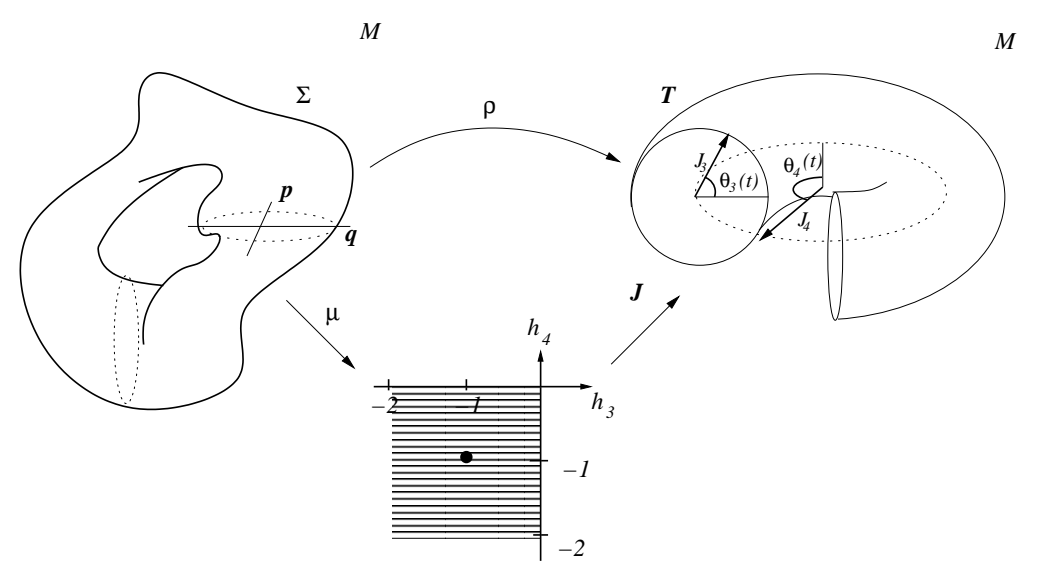

Figura 1.1. Coordenadas acción-ángulo.

Definimos la acción sobre una curva simple cerrada $\gamma$ como $J(\gamma)=\int_{\gamma} \lambda$. Una consecuencia inmediata de la expresión (1.8) es que la acción sobre una curva cerrada simple es invariante bajo simplectomorfismos

Por lo tanto es posible construir un simplectomorfismo para cada sistema Hamiltoniano integrable $\left(M, \omega, X_{H}\right)$ con órbitas periódicas que sólo dependa de los valores de la aplicación momento. Sea $x \in M$ un punto regular y tomemos la imagen bajo la aplicación momento $y=\mu(x) \in \mathfrak{g}^{*}$. Ahora consideremos la fibra $\mu^{-1}(y)$ y supongamos que es una subvariedad compacta, es decir, es un toro Lagrangiano de $M$. Entonces, existe un simplectomorfismo $\rho: M \rightarrow M$ donde el sistema Hamiltoniano transformado sólo depende de la acción de las curvas integrales (simples cerradas). Las coordenadas obtenidas bajo el isomorfismo se conocen como coordenadas acción-ángulo y están 
definidas por

$$
J\left(\gamma_{h_{i}}\right)=\frac{1}{2 \pi} \int_{\gamma_{h_{i}}} \lambda \quad \text { y } \quad \theta\left(h_{i}\right)=\frac{1}{\Omega\left(h_{i}\right)} t+\theta_{0},
$$

donde $\Omega\left(h_{i}\right)=\frac{\partial J}{\partial h_{i}}$. En particular, si se tiene un sistema Hamiltoniano separable, la 1-forma de Liouville $\lambda=p_{i} d q_{i}$ permitirá obtener las coordenadas de acción de manera directa como $n$ sistemas Hamiltonianos de un grado de libertad.

\subsection{Sistemas meCÁnicos.}

Los primeros ejemplos de sistemas Hamiltonianos surgen en los sistemas mecánicos. Aunque en general, estos sistemas se determinan como sistemas Hamiltonianos sobre variedades pre-simplécticas 4, 45, su estudio está más difundido como sistemas Hamiltonianos sobre variedades simplécticas y de Poisson que corresponden a sistemas mecánicos conservativos.

1.7.1. Sistemas mecánicos conservativos. Los sistemas mecánicos conservativos están dados por una ecuación de la forma

$$
\ddot{q}=\nabla V(q),
$$

donde $V: \mathbb{R}^{n} \backslash \Delta \rightarrow \mathbb{R}$ es una función diferenciable y $\Delta \subset \mathbb{R}^{n}$ es el conjunto de singularidades de $V$.

$V$ es llamada la función potencial y la función Hamiltoniana definida de tal forma que el sistema de segundo orden (1.9), se convierta en uno de primer orden, está dada por

$$
H(q, p)=\frac{1}{2}|p|^{2}-V(q) .
$$

Considerando al espacio de configuración $\mathcal{Q}:=\left(\mathbb{R}^{n} \backslash \Delta\right)$ como una variedad abierta con frontera, definimos la variedad simpléctica $\left(M:=T^{*} \mathcal{Q}, \omega\right)$ para ser el espacio (co)fase del sistema Hamiltoniano $\left(M, \omega, X_{H}\right)$ donde el campo vectorial Hamiltoniano $X_{H}$ corresponde a la función (1.10). En este caso, el isomorfismo que manda $T_{q} \mathcal{Q} \rightarrow T_{q}^{*} \mathcal{Q}$ tiene como matriz asociada a $I_{n}$ que es el elemento identidad en $M_{n \times n}(\mathbb{R})$.

De esta forma tenemos una identificación natural de $T \mathcal{Q} \cong T^{*} \mathcal{Q}$ dada por $p=\dot{q}$.

Como la función Hamiltoniana $H: M \rightarrow \mathbb{R}$ es una primera integral para el campo vectorial Hamiltoniano $X_{H}$, ésta define un conjunto invariante (nivel de energía)

$$
\Sigma_{h}=\{(q, p) \in M \mid H(q, p)=h\},
$$

para cada valor fijo de $h \in \operatorname{Img}(H) \subset \mathbb{R}$.

Si $h$ es un valor regular para la función $H$, el conjunto $\Sigma_{h}=H^{-1}(h)$ corresponde a una subvariedad diferenciable de codimensión 1 en $M$. Esta variedad se conoce como la superficie de energía constante o simplemente 
superficie de energía que son variedades de contacto dentro de la variedad $M$.

Consideremos la proyección natural en la base

$$
\begin{aligned}
\pi_{1}: M & \rightarrow \mathcal{Q} \\
(q, p) & \mapsto q,
\end{aligned}
$$

que corresponde a las secciones del espacio cotangente. La imagen de cada superficie de energía bajo la proyección $\pi_{1}\left(\Sigma_{h}\right)$ se le conoce como la región de Hill con energía $h$.

$$
\operatorname{Hill}_{h}:=\pi_{1}\left(\Sigma_{h}\right)
$$

Esto implica que si $\phi(t) \subset \Sigma_{h}$ es una solución del sistema Hamiltoniano con energía $h$, entonces $\pi_{1}\left(\phi\left(t_{0}\right)\right) \in H i l l_{h}$ para todo tiempo $t_{0} \in I \subset \mathbb{R}$.

La frontera de esta región $\partial H i l_{h}$ corresponde a la sección cero y se le conoce como la superficie de velocidad cero, o curva de velocidad cero dependiendo de la dimensión del sistema Hamiltoniano.

En general se llamará conjunto de velocidad cero, y se obtiene cuando $V(q)=-h$.

Las fibras de $M$ restringidas a $\Sigma_{h}$ forman conjuntos convexos, topológicamente equivalentes a esferas $S^{n-1}$ con radio $\sqrt{2(h+V(q))}$, que se obtienen a partir de la relación

$$
0 \leq \frac{1}{2}|p|^{2}=h+V(q)
$$

TeOrema 1.7. Las soluciones de equilibrio del sistema mecánico

$$
\begin{aligned}
\dot{q} & =p, \\
\dot{p} & =\nabla V(q)
\end{aligned}
$$

están dadas por $\left(q_{0}, p_{0}\right)=(0,0)$, y corresponden a los puntos críticos de la función potencial $V$.

Demostración. Debemos notar que si

$$
H=\frac{1}{2}|p|^{2}-V(q),
$$

entonces su gradiente es

$$
\nabla H=(-\nabla V(q), p) .
$$

Para que $h \in \mathbb{R}$ sea un valor regular de $H$, se debe verificar que $d H(x) \neq 0$ para cada $x \in \Sigma_{h}$. por lo tanto, $d H(q, p)=0$ si, y sólo si las siguientes igualdades se cumplen simultáneamente

$$
\begin{aligned}
-\nabla V(q) & =0, \\
p & =0,
\end{aligned}
$$

por consecuencia, si y sólo si $(q, p)$ es un punto de equilibrio para el campo vectorial Hamiltoniano. 
Proposición 1.11. El nivel de energía $\Sigma_{h}$ corresponde a un valor regular para $H$ si y sólo si $\Sigma_{h}$ no contiene puntos de equilibrio del campo vectorial hamiltoniano asociado a $H$.

La demostración de esta proposición puede revisarse en $\mathbf{7 8}$.

Todo sistema mecánico es reversible, en el sentido de que la transformación lineal

$$
\begin{aligned}
& R: \mathbb{R}^{n} \times \mathbb{R}^{n} \rightarrow \mathbb{R}^{n} \times \mathbb{R}^{n} \\
&(q, p) \mapsto(q,-p),
\end{aligned}
$$

deja invariante al sistema.

Es claro que $R$ es un isomorfismo cuya inversa es ella misma, a este tipo de isomorfismos se les llama involución, $R^{2}=R \circ R=I d$.

En efecto

$$
\begin{aligned}
X_{H} \circ R(q, p) & =X_{H}(q,-p) \\
& =\left(H_{p}(q, p),-H_{q}(q,-p)\right) \\
& =(-p, \nabla V(q)) .
\end{aligned}
$$

Por otro lado se tiene que

$$
\begin{aligned}
R \circ X_{H}(q, p) & =R\left(H_{p}(q, p),-H_{q}(q, p)\right) \\
& =\left(H_{p}(q, p), H_{q}(q, p)\right) \\
& =(p,-\nabla V(q)) .
\end{aligned}
$$

De las identidades anteriores podemos ver que

$$
X_{H} \circ R=-R \circ X_{H},
$$

donde se ha sustituido $t \mapsto-t$, y por lo tanto se tiene la simetría $(q, p, t) \mapsto$ $(q,-p,-t)$.

TEOREMA 1.8. Todo sistema mecánico es reversible y admite la involución

$$
(q, p ; t) \mapsto(q,-p ;-t) .
$$

En particular, si una curva solución $(q(t), p(t))$ es tal que $q(t)$ tiene dos puntos de intersección con el conjunto de velocidad cero, entonces $(q(t), p(t))$ es necesariamente periódica.

Enunciamos una variación de una proposición encontrada en [58] que nos permitirá reducir el estudio del espacio fase de sistemas mecánicos a los casos mas relevantes cuando se tiene $h>0, h<0$ y $h=0$.

Proposición 1.12 (Lacomba). Dado un sistema mecánico con potencial homogéneo de grado $\alpha$,

$$
b^{\alpha} V(q)=V(b q)
$$


para cada $\lambda>0$ existe una transformación simpléctica con multiplicador $\lambda$ que lleva la superficie de energía $\Sigma_{h} \mapsto \Sigma_{\lambda h}$, para cualquier $h \in \mathbb{R}$. Un cambio en la escala de tiempo lleva soluciones del sistema original en soluciones del nuevo sistema.

1.7.2. Sistemas Generales Newtonianos. Los sistemas generales Hamiltonianos tienen la forma

$$
\mathcal{M} \ddot{x}+\nabla V(x)=g(t),
$$

donde $x \in \mathcal{Q}:=\left(\mathbb{R}^{n} \backslash \Delta\right)$ con $\Delta$ el conjunto de singularidades de $V(x)$ y $\mathcal{M}$ es una matriz simétrica no singular de orden $n \times n, V: \mathcal{Q} \rightarrow \mathbb{R}$ y $g$ es una función vectorial de la variable real (t) definida en un intervalo $t \in I \subset \mathbb{R}$.

Definamos la variedad $M:=T^{*} \mathcal{Q}$ con la forma simpléctica natural $\omega=$ $\sum_{i} d p_{i} \wedge d q_{i}$, de tal forma que $(M, \omega)$ es una variedad simpléctica. El sistema Hamiltoniano asociado está dado por $\left(M, \omega, X_{H}\right)$ donde $X_{H}=\omega^{\sharp}(d H)$, es decir $i_{X_{H}} \omega=d H$ y $H: M \rightarrow \mathbb{R}$ está definida por

$$
H=\frac{1}{2} p^{T} \mathcal{M}^{-1} p+V(x)-x^{T} g(t),
$$

$\operatorname{con} p \in T_{q}^{*} \mathcal{Q}$.

\subsection{El PROBlema de los $N$ Cuerpos.}

El problema de los $N$ cuerpos es un problema mecánico con $3 N$ ecuaciones de movimiento de segundo grado de la forma

$$
m_{i} \ddot{q}_{i}=\sum_{i, j=1, i<j}^{N} G m_{i} m_{j} \frac{q_{j}-q_{i}}{r_{i j}^{3}},
$$

donde las $m_{i}>0$ son las masas de cada cuerpo, $q_{i} \in \mathbb{R}^{3}$ son las posiciones, $\ddot{q}_{i} \in \mathbb{R}^{3}$ son sus aceleraciones, $r_{i j}=\sqrt{q_{i}-q_{j}}$ son las distancias entre los cuerpos $m_{i}$ y $m_{j}$ con $i, j \in\{1, \cdots, N\}$, y $G$ es la constante de gravitación universal.

Este sistema está definido en $\left(\mathbb{R}^{3 N} \backslash \Delta\right)$ donde el $\Delta$ es el conjunto de singularidades dado por

$$
\Delta=\left\{\left(q_{1}, \cdots, q_{N}\right) \in \mathbb{R}^{3 N} \mid q_{i}=q_{j}, i \neq j, i, j=1, \cdots, N\right\} .
$$

Este conjunto contiene a todas las singularidades por colisión que se pueden presentar en la ecuación (1.11). Adicionalmente, las singularidades que corresponden a escapes de alguno de los cuerpos en tiempo finito también se encuentran en $\Delta$. Esto es consecuencia de un teorema probado por Painlevé en 1895 y que se incluye en 81. En la siguiente sección ofreceremos una breve discusión de las singularidades del problema de $N$-cuerpos.

Consideremos el vector $\mathbf{q}=\left(q_{1}, \cdots, q_{N}\right) \in \mathbb{R}^{3 N}$, que corresponde a las posiciones de los $N$ cuerpos y definamos la variedad $\mathcal{Q}:=\left(\mathbb{R}^{3 N} \backslash \Delta\right)$ abierta, no compacta, con frontera como el espacio de configuración de este sistema. 
El problema de los $N$ cuerpos admite una formulación como sistema Hamiltoniano $\left(M, \omega, X_{H}\right)$ sobre la variedad simpléctica con frontera $(M, \omega)$ donde $M:=T^{*} \mathcal{Q}, \omega=\sum_{i} d p_{i} \wedge d q_{i}$ y $X_{H}$ es el campo vectorial Hamiltoniano asociado a la función

$$
H(\mathbf{q}, \mathbf{p})=\frac{1}{2} \mathbf{p}^{T} \mathcal{M}^{-1} \mathbf{p}-U(\mathbf{q}),
$$

donde $\mathbf{p} \in T_{\mathbf{q}}^{*} \mathcal{Q}$,

$$
U(\mathbf{q})=\sum_{i \neq j} \frac{G m_{i} m_{j}}{r_{i j}},
$$

y $\mathcal{M} \in M_{3 N \times 3 N}\left(\mathbb{R}^{+}\right)$es la matriz de masas dada por

$$
\mathcal{M}=\left(\begin{array}{cccc}
m_{1} I & 0 & \cdots & 0 \\
0 & m_{2} I & & 0 \\
\vdots & & \ddots & \vdots \\
0 & 0 & \cdots & m_{N} I
\end{array}\right)
$$

con $0, I \in M_{3 \times 3}\left(\mathbb{R}^{+}\right)$las matrices nula e identidad respectivamente.

\subsection{Singularidades EN EL PROBlema DE $N$-CUERPOS.}

Seguiremos el tratamiento establecido por Boccaletti y Pucacco en [10].

Definición 1.40. El momento de inercia del problema de $N$-cuerpos, es la distribución de los cuerpos con respecto de su centro de masa (o centro de rotación) y se determina por la expresión

$$
I=\sum_{i=1}^{N} m_{i} r_{i}^{2}
$$

donde $r_{i}$ es la distancia del $i$-ésimo cuerpo al centro de masa del sistema.

Denotemos por

$$
r=\operatorname{mín}_{1 \leq i<j \leq n} r_{i j} \quad \mathrm{y} \quad R=\operatorname{máx}_{1 \leq i<j \leq n} r_{i j},
$$

a la mínima y máxima distancias entre las parejas de los $N$ cuerpos.

El objetivo de los siguientes cálculos es dar una estimación de los valores de $r$ y $R$ en términos de la función potencial $U$ y del momento de inercia $I$ definidos en (1.14) y (1.15) respectivamente.

Fijemos un valor de $k \in\{1, \ldots, N\}$ y desarrollemos el cuadrado de las distancias entre los cuerpos $k$-ésimo y $j$-ésimo. Multiplicando el cuadrado de esa distancia por la masa del $j$-ésimo cuerpo y sumando en $j$ tenemos la identidad

$$
\sum_{j=1}^{N} m_{j}\left(r_{j}-r_{k}\right)^{2}=\sum_{j=1}^{N} m_{j} r_{j}^{2}-2 r_{k} \sum_{j=1}^{N} m_{j} r_{j}+\sum_{j=1}^{N} m_{j} r_{k}^{2}
$$


Si las coordenadas toman como origen al centro de masa, el término cruzado es nulo así que

$$
\sum_{j} m_{j}\left(r_{j}-r_{k}\right)^{2}=I+M r_{k}
$$

Multiplicando por $m_{k}$ y sumando todos los términos en $k$ tenemos que

$$
\sum_{k} \sum_{j} m_{k} m_{j} r_{j k}^{2}=2 M I
$$

o de manera equivalente

$$
\sum_{1 \leq j<k \leq N} m_{k} m_{j} r_{j k}^{2}=M I
$$

Como $r \leq r_{j k}$ para toda $j, k=1, \ldots, N$ con $j \neq k$ entonces tenemos que

$$
U \leq \sum_{1 \leq j<k \leq N} \frac{m_{j} m_{k}}{r}
$$

y como

$$
\sum_{1 \leq j<k \leq N} m_{j} m_{k}=\frac{M^{2}}{2}
$$

tenemos que $U \leq \frac{M^{2}}{2 r}$. Ahora denotemos por

$$
m=\min _{1 \leq j \leq N}\left\{m_{j}\right\}
$$

el valor de la masa más pequeña de los $N$ cuerpos. Esto nos indica que

$$
U \geq \sum_{1 \leq j<k \leq N} \frac{m_{j} m_{k}}{r_{j k}} \geq \frac{m^{2}}{r_{j k}}
$$

para todo $j, k=1, \ldots, N$, de donde se deduce inmediatamente que $\frac{m^{2}}{r} \leq U$. Entonces existen dos constantes positivas $a=m^{2}$ y $b=M^{2} / 2$ tal que

$$
\frac{a}{U} \leq r \leq \frac{b}{U}
$$

Por otro lado, se tiene que $R^{2} \leq \sum_{j<k} r_{j k}^{2}$ y utilizando la expresión (1.20) podemos ver que

$$
\frac{m^{2}}{M} R^{2} \leq \frac{m^{2}}{M} \sum_{j<k} r_{j k}^{2} \leq \frac{1}{M} \sum_{j<k} m_{j} m_{k} r_{j k}^{2}=I .
$$

Además tenemos que

$$
I=\frac{1}{M} \sum_{j<k} m_{j} m_{k} r_{j k}^{2} \leq \frac{1}{M} \sum_{j<k} m_{j} m_{k} R^{2}=\frac{1}{2} M R^{2},
$$


de forma que

$$
\frac{m^{2}}{M} R^{2} \leq I \leq \frac{1}{2} M R^{2}
$$

De manera análoga, tenemos dos constantes positivas $a^{\prime}=\sqrt{2 / M} \mathrm{y}$ $b^{\prime}=\sqrt{M} / m$ tal que

$$
a^{\prime} \sqrt{I} \leq R \leq b^{\prime} \sqrt{I} .
$$

Por lo tanto, $\frac{1}{U}$ es una estimación para el valor de $r$ mientras que $\sqrt{I}$ lo es para el valor de $R$.

Veamos ahora el resultado obtenido por Painlevé acerca de la existencia de singularidades en el problema de $N$ cuerpos.

Teorema 1.9 (Painlevé 1897). Definamos por

$$
r(t)=\min _{j<k}\left\{r_{j k}\right\}
$$

a la más pequeña de las distancias mutuas al instante $t$. Si el movimiento es regular para $t<t_{1}$ pero no en $t_{1}$, entonces $r(t) \rightarrow 0$ cuando $t \rightarrow t_{1}$ : es decir que para $t$ suficientemente cerca de $t_{1}, r(t)$ es inferior a toda cantidad $\epsilon$ dada.

El teorema anterior nos indica que una condición necesaria y suficiente para tener una singularidad en el instante $t=t_{1}$ es que $r \rightarrow 0$ cuando $t \rightarrow t_{1}$. Sin embargo, Painlevé también observó que la condición de que $r \rightarrow 0$ cuando $t \rightarrow t_{1}$ no implica que la singularidad se deba a un colisión 81. pp. 583]. De hecho, podría suceder que algunas de las distancias $r_{j k}$ oscilen de tal forma que

$$
\liminf r_{j k}=0 \quad \text { y } \quad \operatorname{límsup} r_{j k}>0,
$$

mientras que $r \rightarrow 0$. Es decir, que $r$ puede tender a cero y se puede deber a una singularidad sin colisión. Painlevé llamó pseudocolisiones a estas singularidades siguiendo una sugerencia hecha por Poincaré [10, 81]. Para el caso en que $N=3$, Painlevé obtuvo el siguiente resultado:

Teorema 1.10 (Painlevé 1897). En el problema de 3-cuerpos, todas las singularidades son colisiones.

Sin embargo, el problema de determinar si existían pseudocolisiones para $N \geq 4$ quedó sin respuesta hasta 1988 cuando Z. Xia demostró la existencia de singularidades sin colisión para $N=5$ [104.

En 1908 Von Zeipel da un paso importante en la caracterización de las singularidades del problema de $N$-cuerpos al demostrar el siguiente resultado:

TeOrema 1.11 (Von Zeipel 1908). Si alguno de los puntos materiales no tiende a una posición límite finita cuando $t \rightarrow t_{1}$, entonces necesariamente

$$
\lim _{t \rightarrow t_{1}} R \rightarrow \infty \text {. }
$$


Este resultado nos indica que cuando una singularidad es debida a una colisión, el sistema permanece acotado y la única forma de tener pseudocolisiones es que el sistema "explote" al infinito en tiempo finito. El resultado anterior fue demostrado por Von Zeipel en [106 y en 1986 R. McGehee dió una demostración alternativa en un lenguaje más actual $\mathbf{7 2}$.

El comportamiento de los sistemas con este tipo de singularidades tienen fuertes oscilaciones cuando la solución se aproxima a $\Delta$ [66 sec 3.2.2], lo que permite ver que son singularidades esenciales no son removibles.

\subsection{REDUCCIÓN SIMPLÉCTICA DEL PROBlEMA DE $N$ CUERPos.}

Para realizar la reducción del sistema Hamiltoniano, debemos considerar la acción de un grupo de Lie $G$ que actúe de manera Hamiltoniana sobre el fibrado cotangente $T^{*} \mathbb{R}^{3 N}$. Para ello se necesita considerar una aplicación momento construida por las integrales conocidas del problema de $N$ cuerpos.

1.10.1. Momento Lineal. La función potencial $V: M \rightarrow \mathbb{R}$ es independiente de los ejes coordenados ya que solo depende de las distancias mutuas de los cuerpos. Por lo tanto, consideremos el grupo de Lie $G_{1}=\mathbb{R}^{3}$, con la adición como operación del grupo. Ahora consideremos la acción de $G_{1}$ sobre $M$ de la forma

$$
\begin{aligned}
R_{z}: G_{1} \times M & \rightarrow M \\
(z,(\mathbf{q}, \mathbf{p})) & \mapsto(\mathbf{q}+\mathbf{z}, \mathbf{p}),
\end{aligned}
$$

donde $\mathbf{z}=(z, \cdots, z) \in \mathbb{R}^{3 N}$ y que consiste en trasladar la posición $q_{i}$ de cada cuerpos a $q_{i}+z$ para $i=1, \cdots, N$. Aplicando la acción $8 R_{z}$ a $X_{H}$ tendremos en coordenadas

$$
\begin{aligned}
\dot{q}_{i} & =m_{i}^{-1} p_{i}, \\
\dot{p}_{i} & =2 G \sum_{j<i} m_{i} m_{j} \frac{\left(q_{j}+z\right)-\left(q_{i}+z\right)}{\left|\left(q_{j}+z\right)-\left(q_{i}+z\right)\right|^{3}} \\
& =2 G \sum_{j<i} m_{i} m_{j} \frac{q_{j}-q_{i}}{\left|q_{j}-q_{i}\right|^{3}},
\end{aligned}
$$

para $i=1, \cdots, N$ y $q_{i}, p_{i} \in \mathbb{R}^{3}$. Por lo tanto vemos que $R_{z}^{*}\left(X_{H}\right)=X_{H} \mathrm{y}$ como $R_{-z}=L_{z}$, entonces $X_{H}$ es invariante bajo la acción de $G_{1}$. Es decir, que el problema de $N$ cuerpos es invariante bajo translaciones.

Esto indica que debe existir una constante de movimiento o integral primera que obtenemos al buscar las variaciones del momento lineal bajo la

\footnotetext{
${ }^{8}$ Elegimos aplicar una translación derecha $R_{z}$ para evitar confusiones con la notación $L_{z}$ de las translaciones izquierdas y $L_{X}$ de la derivada de Lie
} 
acción del grupo de Lie. Por lo tanto tenemos

$$
\sum_{i=1}^{N} \dot{p}_{i}=0,
$$

que se conoce como la conservación del momento lineal. Integrando con respecto del tiempo tenemos

$$
\sum_{i=1}^{N} p_{i}=c_{1},
$$

donde $c_{1} \in \mathbb{R}^{3}$ es un vector constante. Considerando la identidad $m \dot{q}_{i}=p_{i}$ para $i=1, \cdots, 4$, tendremos

$$
\sum_{i=1}^{N} m_{i} q_{i}=c_{1} t+c_{0},
$$

donde $c_{0} \in \mathbb{R}^{3}$ es también un vector constante y $\mathcal{M}=\sum_{i=1}^{N} m_{i} q_{i}$ es el centro de masa del sistema.

TeOrema 1.12. Si $N$ cuerpos están sujetos únicamente a sus fuerzas de atracción mutua, entonces su centro de masa se mueve en una línea recta con velocidad uniforme.

Ahora realicemos la reducción del espacio fase mediante reducción simpléctica.

$\left(M, \omega, X_{H}, G\right)$ forma un sistema Hamiltoniano con una $G$-simetría bajo la acción de $G=\left(\mathbb{R}^{3},+\right)$ por translaciones. El momento lineal $\mathcal{P}=\sum_{i} p_{i}$ es una integral primera y consideramos una aplicación momento

$$
\begin{aligned}
\mu_{1}: T^{*} \mathbb{R}^{3 N} & \rightarrow\left(\mathbb{R}^{3}\right)^{*} \\
(\mathbf{q}, \mathbf{p}) & \mapsto \mathcal{P}=\sum_{i} p_{i},
\end{aligned}
$$

donde $\mathbf{q}, \mathbf{p} \in \mathbb{R}^{3 N}, p_{i} \in \mathbb{R}^{3}$ y $\mathbf{p}=\left(p_{1}, \cdots, p_{N}\right)$.

Para cada $p \in \mathfrak{g}^{*}=\left(\mathbb{R}^{3}\right)^{*}$ su conjunto de nivel

$$
M_{p}=\mu_{1}^{-1}(p) \cong \mathbb{R}^{3 N} \times\left(\mathbb{R}^{3(N-1)}\right)^{*},
$$

es un subespacio isotrópico de $\operatorname{dim} M_{p}=\operatorname{dim} M-\operatorname{dim} \mathfrak{g}^{*}$. Además, como el álgebra de Lie de $\mathbb{R}_{+}^{3}$ es trivial entonces la acción coadjunta es invariante, por lo tanto el grupo de isotropías $G_{p}$ es todo $R_{+}^{3}$.

Aplicando el teorema de la sección 1.5 podemos hacer la reducción simpléctica del sistema original y tener

$$
\begin{aligned}
\tilde{M}_{p} & =M_{p} / G_{p} \\
& =\left(\mathbb{R}^{3 N} \times\left(\mathbb{R}^{3(N-1)}\right)^{*}\right) / \mathbb{R}_{+}^{3} \\
& \cong T^{*}\left(\mathbb{R}^{3(N-1)}\right) .
\end{aligned}
$$


En particular consideramos $c_{1}=c_{0}=(0,0,0)$ lo que indica que el centro de masa $\mathcal{M}=0$ se encuentra fijo en el origen. Denotemos por $\tilde{M}_{0}=$ $T^{*}\left(\mathbb{R}^{3(N-1)}\right), \tilde{\omega}=\left.\omega\right|_{\tilde{M}_{0}} \mathrm{y}$

$$
\tilde{H}=\left.H\right|_{\tilde{M}_{0}}: \tilde{M}_{0} \rightarrow \mathbb{R}
$$

tenemos el siguiente resultado:

Teorema 1.13. El sistema Hamiltoniano reducido $\left(\tilde{M}_{0}, \tilde{\omega}, X_{\tilde{H}}\right)$ es la restricción del problema de $N$-cuerpos a la condición

$$
\sum_{i} m_{i} q_{i}=0 .
$$

1.10.2. Momento Angular. El problema de $N$-cuerpos tiene otra simetría que son las rotaciones del sistema. Entonces podemos considerar la acción de un segundo grupo de Lie $G_{2}=S O(3)$ que actuará por rotaciones de la forma

$$
\begin{aligned}
r_{A}: G_{2} \times M & \rightarrow M \\
(A,(\mathbf{q}, \mathbf{p})) & \mapsto\left(A q_{1}, \cdots, A q_{N}, A p_{1}, \cdots, A p_{N}\right) .
\end{aligned}
$$

Tomemos una matriz de rotación $A \in S O(3)$ y apliquemos la acción al campo vectorial $X_{H}$

$$
\begin{aligned}
\dot{q}_{i} & =A p_{i}, \\
\dot{p}_{i} & =2 G \sum_{j<i} m_{i} m_{j} \frac{A q_{j}-A q_{i}}{\left|A q_{j}-A q_{i}\right|^{3}} .
\end{aligned}
$$

La norma euclidiana es invariante bajo rotaciones, $\left|A\left(q_{i}-q_{j}\right)\right|=\left|q_{i}-q_{j}\right|$ lo que implica

$$
\begin{aligned}
\dot{q}_{i} & =A p_{i}, \\
\dot{p}_{i} & =A\left(2 G \sum_{j<i} m_{i} m_{j} \frac{q_{j}-q_{i}}{\left|q_{j}-q_{i}\right|^{3}}\right) .
\end{aligned}
$$

De manera equivalente tenemos que $r_{A}^{*}\left(X_{H}\right)=A\left(X_{H}\right)$, lo que indica que el campo conmuta con la acción del grupo de Lie. Cada elemento $A \in S O(3)$ puede identificarse con un vector $v \in \mathbb{R}^{3}$ que indica el eje de rotación y la norma del vector se asocia a la velocidad angular mediante el isomorfismo $A \cong x$ definido por

$$
\left(\begin{array}{ccc}
0 & -a & b \\
a & 0 & -c \\
-b & c & o
\end{array}\right) \cong\left(\begin{array}{l}
a \\
b \\
c
\end{array}\right) .
$$

TeOrema 1.14. Si $N$ cuerpos están sujetos únicamente a su fuerzas de atracción mutuas, la suma de los productos de las masas y la razón de cambio de las proyecciones de sus áreas son constantes. Es decir,

$$
\sum_{i} p_{i} \times q_{i}=c_{0}
$$


Como vemos que el campo vectorial Hamiltoniano $X_{H}$ conmuta bajo la acción del grupo de Lie $G=S O(3)$ entonces existe una aplicación momentd 9 $\mu_{2}: M \rightarrow \mathfrak{s o}^{*}(3)$ definido por

$$
\begin{aligned}
\mu_{2}(\mathbf{q}, \mathbf{p}) & =\mathbf{p} \times \mathbf{q} \\
& =\sum_{i} p_{i} \times q_{i} \\
& =p_{0},
\end{aligned}
$$

donde $p_{0} \in \mathfrak{s o}^{*}(3)$. En este caso, tenemos que los coeficientes característicos del álgebra de Lie son

$$
\begin{aligned}
& c_{1,2}^{3}=c_{2,3}^{1}=c_{3,1}^{2}=1, \\
& c_{i, j}^{k}=0 \quad \text { en los otros casos. }
\end{aligned}
$$

Esto significa que el álgebra de Lie no es conmutativa y por lo tanto el grupo de isotropía es un subgrupo propio de $S O(3)$. Se tiene

$$
G_{p}=S O(2) \text {. }
$$

Sea $p \in \mathfrak{s o}(3)^{*} \cong \mathbb{R}^{3}$, entonces $G_{p}$ corresponde a las rotaciones del sistema alrededor del vector $p \in \mathbb{R}^{3}$ que están dadas por $A \cdot p$ con $A \in S O(2)$. En coordenadas tenemos

$$
A \cdot p=\left(\begin{array}{ccc}
\cos \theta & -\sin \theta & 0 \\
\sin \theta & \cos \theta & 0 \\
0 & 0 & 1
\end{array}\right)\left(\begin{array}{l}
p_{1} \\
p_{2} \\
p_{3}
\end{array}\right) .
$$

y por lo tanto $A$ tiene dimensión 1. A la reducción del espacio fase por las simetrías de los grupos $S O(3)$ y $S O(2)$ se le conoce clásicamente como eliminación de los nodos $\mathbf{2 8}$.

Consideremos un conjunto de nivel de la aplicación momento $M_{p}=$ $\mu_{2}^{-1}(p)$ para $p \in \mathfrak{s o}^{*}(3)$. Ésta es una variedad suave tal que $\operatorname{dim} M_{p}=$ $\operatorname{dim} M-\operatorname{dim} \mathfrak{s o}^{*}(3)$, es decir, de codimensión 3 . Y considerando el cociente de esta subvariedad con el grupo de isotropía $G_{p}=S O(2)$ que tiene dimensión 1 tenemos

$$
\begin{aligned}
\tilde{M}_{p} & =M_{p} / G_{p} \\
& =\mu_{2}^{-1}(p) / S O(2) .
\end{aligned}
$$

TeOrema 1.15. El sistema Hamiltoniano reducido $\left(\tilde{M}_{p}, \tilde{\omega}, X_{\tilde{H}}\right)$ es la restricción del problema de $N$-cuerpos a la condición

$$
\sum_{i} p_{i} \times q_{i}=c_{0}
$$

Finalmente, en el problema general de los $N$-cuerpos se ha podido reducir el espacio fase de un espacio de dimensión $6 N$ a un espacio de dimensión $6 N-10$. Las primeras 6 dimensiones corresponden a la reducción simpléctica del momento lineal (que corresponden a 6 integrales primeras), mientras

\footnotetext{
${ }^{9}$ Abusando de la notación, volveremos a denotar por $M$ al espacio fase reducido $\tilde{M}_{p}$
} 
que las últimas cuatro pertenecen a la reducción por simetría del momento angular.

Corolario 2. El problema de dos cuerpos es completamente integrable.

Este problema se encuentra definido por un sistema Hamiltoniano con un espacio fase reducido de dimensión $6 N-10=2$. Por lo tanto es un Hamiltoniano con un grado de libertad y una primera integral $H$.

1.10.3. LA INTEGRAL DE ENERGÍA. Integramos esta sección para tener un estudio completo sobre la obtención de las 10 integrales conocidas del problema de $N$-cuerpos. Para ello utilizaremos las ecuaciones originales escritas a partir de la segunda ley de Newton.

Consideremos las ecuaciones de movimiento del problema de $N$ cuerpos de la forma

$$
m_{i} \ddot{q}_{i}=\frac{\partial U}{\partial q_{i}}, \quad i=1, \cdots, N
$$

Escrito en componentes en $\mathbb{R}^{3}$ tendremos

$$
m_{i} \ddot{x}_{i}=\frac{\partial U}{\partial x_{i}}, \quad m_{i} \ddot{y}_{i}=\frac{\partial U}{\partial y_{i}}, \quad m_{i} \ddot{z}_{i}=\frac{\partial U}{\partial z_{i}}, \quad i=1, \cdots, N .
$$

Multiplicando cada ecuación por $\dot{x}_{i}, \dot{y}_{i}, \dot{z}_{i}$, respectivamente, y sumando elemento a elemento con respecto de $i$ tenemos

$$
\sum_{i} m_{i}\left(\ddot{x}_{i} \dot{x}_{i}+\ddot{y}_{i} \dot{y}_{i}+\ddot{z}_{i} \dot{z}_{i}\right)=\sum_{i}\left(\frac{\partial U}{\partial x_{i}} \dot{x}_{i}+\frac{\partial U}{\partial y_{i}} \dot{y}_{i}+\frac{\partial U}{\partial z_{i}} \dot{x}_{i}\right) .
$$

El potencial $U$ es función de las variables $x_{i}, y_{i}, z_{i}$ únicamente, así que el miembro derecho de la igualdad es la derivada total de $U$ con respecto del tiempo $t$. Integrando ambos miembros de la ecuación tendremos

$$
\frac{1}{2} \sum_{i} m_{i}\left(\dot{x}_{i}^{2}+\dot{y}_{i}^{2}+\dot{z}_{i}^{2}\right)=U+h,
$$

donde $h$ es una constante de integración. Veamos que el miembro izquierdo de la ecuación corresponde a la energía cinética de todo el sistema y el miembro de la derecha corresponde a la energía potencial más una constante. Reescribimos la ecuación de la forma

$$
h=\frac{1}{2} \sum_{i} m_{i}\left(\dot{x}_{i}^{2}+\dot{y}_{i}^{2}+\dot{z}_{i}^{2}\right)-U .
$$

Así tenemos el siguiente resultado

TeOREma 1.16. En un sistema de $N$ cuerpos sujetos exclusivamente a sus fuerzas de atracción mutua, la suma de las energías cinética y potencial es constante. 
1.10.4. Existencia DE otras integrales. Las diez integrales del sistema de $N$ cuerpos (6 del momento lineal, 3 del momento angular y 1 de la energía), son las únicas integrales conocidas del problema general y durante muchos años se cuestionó si existía alguna integral adicional. Bruns demostró que el problema en coordenadas rectangulares no tiene otra integral algebraica. Esto no elimina la posibilidad de encontrar otra integral algebraica en otro sistema de coordenadas.

Por su parte, Poincaré demostró que utilizando como referencia los elementos de las órbitas de cada cuerpo, el problema de tres cuerpos no admite otra integral uniforme trascendente, aún cuando las masas de dos de los cuerpos sean muy pequeñas comparadas con la masa del tercer cuerpo. Nuevamente, esto no indica que no exista otra integral de dicho tipo en otro marco de referencia o con otras coordenadas.

Sin embargo, debe considerarse que la importancia práctica de estos resultados es que han sido establecidos bajo condiciones particulares lo que no implica su validez absoluta. 


\section{CAPÍTULO 2}

\section{El PROBlema De Sitnikov CON $2+2$ CUERPOS}

En este capítulo presentamos los antecedentes y la descripción del problema que será estudiado en el resto de este trabajo. Como el problema propuesto es una extensión del problema de Sitnikov iniciaremos con una breve descripción de dicho problema. Posteriormente presentaremos el problema con 4 cuerpos, sus ecuaciones de movimiento y un sistema regularizado que nos permitirá estudiar el problema cerca de sus singularidades. Finalizamos este capítulo presentando un caso reducido bajo simetrías que es integrable por cuadraturas.

\subsection{El PROBlema de Sitnikov.}

El problema de Sitnikov es un caso especial del problema restringido de tres cuerpos donde dos cuerpos masivos con masas $m_{1}=m_{2}=\frac{1}{2}$ evolucionan en órbitas Keplerianas alrededor de su centro de masas y un cuerpo de masa infinitesimal $m_{3} \approx 0$ se mueve sobre la recta perpendicular al plano donde evolucionan los cuerpos masivos y que pasa por su centro de masas. La atracción que ejerce el cuerpo infinitesimal sobre los primarios es despreciable por lo que no perturba la evolución de éstos sobre las órbitas Keplerianas. El problema de Sitnikov consiste en determinar la dinámica del cuerpo infinitesimal (secundario) bajo la atracción de los cuerpos masivos (primarios) con potencial gravitacional Newtoniano.

La ecuación de movimiento para el cuerpo secundario en forma Newtoniana es

$$
m_{3} \ddot{z}=-\frac{2 G m_{1} m_{3}}{\sqrt{z^{2}+R(t)^{2} / 4}}
$$




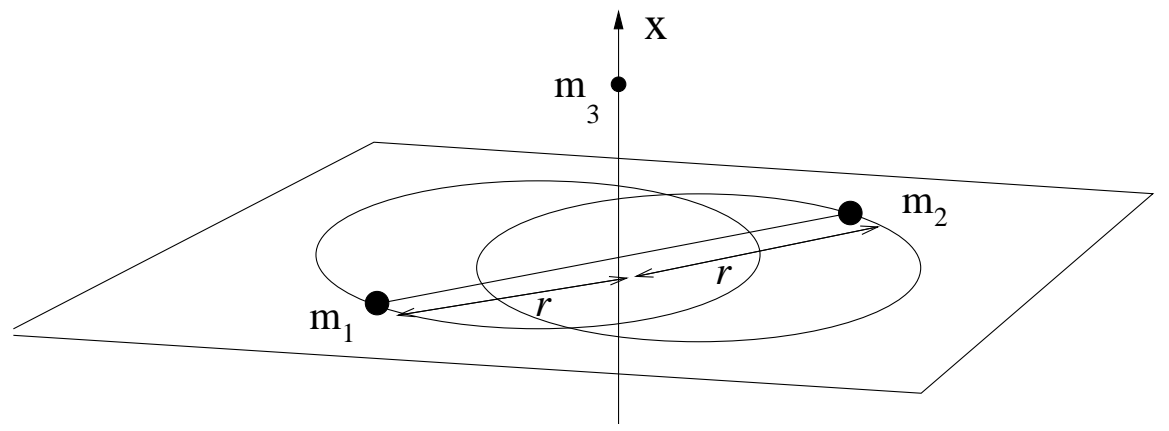

Figura 2.1. El problema de Sitnikov.

donde $G$ es la constante de gravitación universal $m_{1}=m_{2}$ es la masa de los cuerpos primarios y $R(t)$ es la distancia entre ellos. Como $0<m_{3}$ cancelamos este término de ambos lados de la ecuación diferencial y consideramos el momento $p_{z}=\dot{z}$ para obtener un sistema Hamiltoniano a partir de (2.1) cuya función Hamiltoniana es

$$
H\left(z, p_{z}, t\right)=\frac{1}{2} p_{z}^{2}-\frac{2 G m_{1}}{\sqrt{z^{2}+R(t)^{2} / 4}} .
$$

La solución para la distancia $R(t)$ esta dada por la anomalía excéntrica $\psi \in[0,2 \pi]$ mediante

$$
R=a(1-e \cos \psi)
$$

que satisface la ecuación de Kepler

$$
\psi-\sin \psi=\frac{2 \pi}{T} t
$$

donde $T$ es el periodo orbital [13, 47, 80, 85. La tercera ley de Kepler afirma que el cuadrado del periodo de revolución es proporcional al cubo del semieje mayor [41, pp 198-201], por lo tanto

$$
\frac{T^{2}}{a^{3}}=\frac{4 \pi^{2}}{2 G m_{1}}
$$

donde $a$ es el semieje mayor de las elípses que describen las órbitas de los primarios. Adicionalmente, se tiene que el momento angular $l$ de los cuerpos primarios cumple la relación

$$
a\left(1-e^{2}\right)=\frac{2 l^{2}}{2 G m_{1}^{3}} .
$$




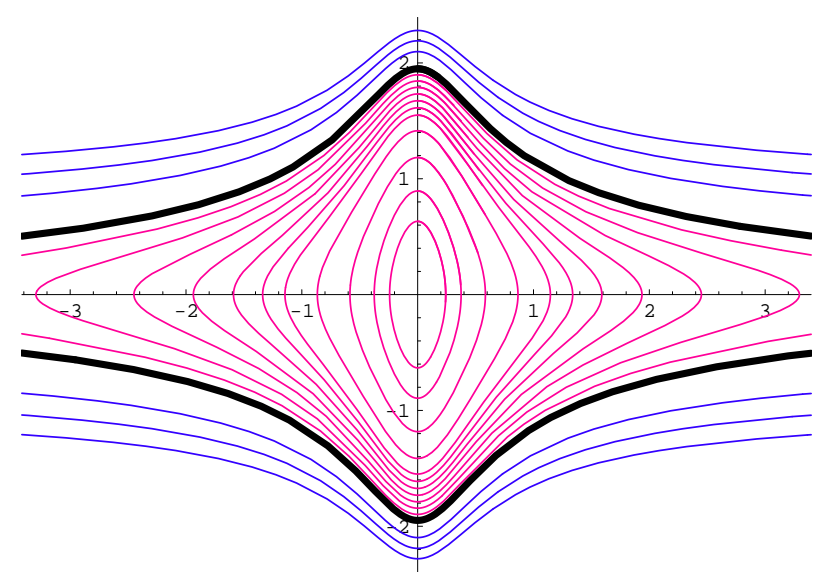

FigurA 2.2. El espacio fase del problema circular de Sitnikov.

Utilizando la anomalía excéntrica como la nueva variable independiente, el cambio de variables

$$
q=\frac{z}{\alpha}, \quad p=\frac{p_{z}}{\alpha} \sqrt{\frac{\alpha^{3}}{2 G m_{1}}}, \quad T=\sqrt{\frac{2 G m_{1}}{\alpha^{3}}} t .
$$

permite escribir la función Hamiltoniana (2.2) de la forma

$$
H(q, p, t)=\frac{1}{2} p^{2}-\frac{1}{\sqrt{q^{2}+r(t)^{2}}},
$$

donde $r(t)=\frac{1}{2}(1-e \cos (\psi(t)))$ y $\alpha=a\left(1-e^{2}\right)$.

Las nuevas variables dadas en (2.7) no poseen dimensiones físicas al igual que $r(t)=R(\psi(t)) / \alpha$ y la función Hamiltoniana (2.8) 47.

La función (2.8) depende del parámetro $e \in[0,1)$ que corresponde a la excentricidad de las órbitas de los cuerpos primarios. Cuando $e=0$ los primarios se mueven en órbitas circulares y por lo tanto (2.8) define el problema circular de Sitnikov (también conocido como problema de MacMillan 65]). El caso circular del Problema de Sitnikov tiene un grado de libertad ya que es autónomo y por lo tanto es integrable por cuadraturas.

Las órbitas del problema circular de Sitnikov son descritas por los niveles de energía $H=h$, donde $h \in[-2, \infty)$. El espacio fase $(q, p)$ está foliado por diferentes tipos de curvas [63]:

1. si $h<-2$ no existen órbitas,

2. si $h=-2$ tenemos un punto de equilibrio,

3. si $-2<h<0$ tenemos órbitas periódicas,

4. si $h=0$ tenemos dos órbitas parabólicas (es decir, dos órbitas que vienen y se escapan al infinito con velocidad nula),

5. si $h>0$ tenemos dos órbitas hiperbólicas (es decir, dos órbitas que vienen y se escapan al infinito con velocidad positiva). 
Si el valor de la excentricidad $e \in(0,1)$, entonces la función (2.8) corresponde al problema elíptico de Sitnikov. En este caso, el sistema Hamiltoniano es no autónomo y se ha demostrado que es no integrable [2, 33, 77.

\subsection{El problema De Sitnikov CON $2+2$ CUERPOS.}

En este trabajo se propone una extensión del problema de Sitnikov en donde se consideran cuatro cuerpos en una configuración de tipo $2+2$. Lo anterior significa que se tendrán dos cuerpos primarios y dos secundarios bajo las condiciones descritas a continuación. Los dos cuerpos primarios de masas $m_{1}=m_{2}=1 / 2$ evolucionan en órbitas Keplerianas en el plano $\Pi$ con centro de masa fijo en el origen, de manera equivalente al problema de Sitnikov clásico. Adicionalmente se tienen dos cuerpos secundarios de masas $m_{3}$ y $m_{4}$ (con $\left.m_{3} \approx m_{4} \ll 1 / 2\right)$ que evolucionan sobre una recta perpendicular al plano $\Pi$ que pasa por el centro de masa de los cuerpos primarios. El problema de $2+2$ cuerpos consiste en determinar la dinámica de los dos cuerpos secundarios bajo la atracción gravitacional de los primarios cumpliendo las leyes de Newton.

A este problema lo denominaremos el problema de Sitnikov con 2+2 cuerpos o, de manera equivalente, el problema de Sitnikov con 4 cuerpos en configuración $2+2$.

Elegimos un sistema de coordenadas ortogonales $(x, y, z)$ de tal forma que las órbitas de los primarios se encuentren en el plano $X Y$ y la dinámica de los cuerpos secundarios se desarrolle sobre el eje $Z$. Utilizando un sistema de coordenadas adimensionales equivalente a (2.7) para cada secundario establecemos la siguiente

Definición 2.1. El espacio de posiciones o espacio de configuración para el problema de Sitnikov con 2+2 cuerpos está dado por el conjunto

$$
\mathcal{Q}=\left\{\left(q_{3}, q_{4}\right) \in \mathbb{R}^{2} \mid q_{3}>q_{4}\right\}
$$

donde $q_{3}$ es la posición del cuerpo con masa $m_{3}$ y $q_{4}$ es la posición del cuerpo con masa $\mathrm{m}_{4}$.

El potencial del problema es una función cuyo dominio es el espacio de configuración $\bar{V}: \mathcal{Q} \rightarrow \mathbb{R}$ y que está dada por

$$
\bar{V}\left(q_{3}, q_{4}\right)=\frac{m_{3}}{\sqrt{q_{3}^{2}+r(t)^{2}}}+\frac{m_{4}}{\sqrt{q_{4}^{2}+r(t)^{2}}}+\frac{m_{3} m_{4}}{q_{3}-q_{4}} .
$$

Un breve análisis a la función (2.10) nos permite ver que el conjunto de configuraciones prohibidas o singularidades $\Delta=\left\{\left(q_{3}, q_{4}\right) \in \mathbb{R}^{2} \mid q_{3}=q_{4}\right\}$ es un subconjunto de la frontera del espacio de configuraciones $\Delta \subset \partial \mathcal{Q}$ y corresponde a las colisiones entre los secundarios.

Genéricamente las masas de los secundarios $m_{3} \neq m_{4}$ son distintas, sin embargo, ambas son del mismo orden por lo que podemos reescribirlas 


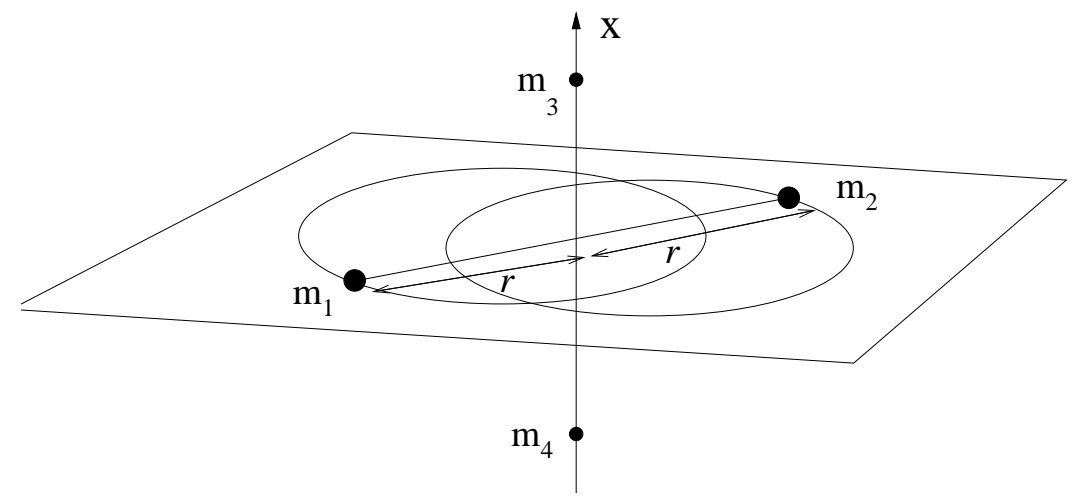

Figura 2.3. El problema de Sitnikov de 2+2 cuerpos

mediante los parámetros

$$
m=\frac{m_{3}+m_{4}}{2} \quad \text { y } \quad \epsilon=\frac{m_{3}-m_{4}}{m_{3}+m_{4}},
$$

de donde obtenemos $m_{3}=(1+\epsilon) m$ and $m_{4}=(1-\epsilon) m$ para $0<m \ll 1 / 2$ y con $\epsilon \in(-1,1)$.

El nuevo potencial utilizando $m$ y $\epsilon$ es

$$
\bar{V}\left(q_{3}, q_{4}\right)=\frac{(1+\epsilon) m}{\sqrt{q_{3}^{2}+1 / 4}}+\frac{(1-\epsilon) m}{\sqrt{q_{4}^{2}+1 / 4}}+m^{2} \frac{1-\epsilon^{2}}{q_{3}-q_{4}},
$$

y satisface la ecuación diferencial de segundo orden $\overline{\mathcal{M}} \ddot{\mathbf{q}}+\frac{\partial \bar{V}}{\partial \mathbf{q}}=0$, donde

$$
\overline{\mathcal{M}}=\left(\begin{array}{cc}
(1+\epsilon) m & 0 \\
0 & (1-\epsilon) m
\end{array}\right)
$$

es la matriz de masas de los cuerpos secundarios.

Como $m \neq 0$ podemos definir la función $V\left(q_{3}, q_{4}\right)=\frac{1}{m} \bar{V}\left(q_{3}, q_{4}\right)$ y la matriz $\mathcal{M}=\frac{1}{m} \overline{\mathcal{M}}$ y reescribir el sistema equivalente como $\mathcal{M} \ddot{\mathbf{q}}+\frac{\partial V}{\partial \mathbf{q}}=0$.

En coordenadas tenemos el nuevo sistema de la forma

$$
\begin{aligned}
& (1+\epsilon) \ddot{q}_{3}+\frac{(1+\epsilon) q_{3}}{\left(q_{3}^{2}+r(t)^{2}\right)^{\frac{3}{2}}}+m \frac{1-\epsilon^{2}}{\left(q_{3}-q_{4}\right)^{2}}=0, \\
& (1-\epsilon) \ddot{q}_{3}+\frac{(1-\epsilon) q_{4}}{\left(q_{4}^{2}+r(t)^{2}\right)^{\frac{3}{2}}}-m \frac{1-\epsilon^{2}}{\left(q_{3}-q_{4}\right)^{2}}=0 .
\end{aligned}
$$

Introducimos los momentos

$$
p_{3}=(1+\epsilon) \dot{q}_{3}, \quad \text { y } \quad p_{4}=(1-\epsilon) \dot{q}_{4},
$$

que nos permitirán escribir la formulación Hamiltoniana del problema mediante la función

$\left(2.14 \not H I(\mathbf{q}, \mathbf{p})=\mathbf{p}^{T} \mathcal{M}^{-1} \mathbf{p}-\frac{1+\epsilon}{\sqrt{q_{3}^{2}+r(t)^{2}}}-\frac{1-\epsilon}{\sqrt{q_{4}^{2}+r(t)^{2}}}-m \frac{1-\epsilon^{2}}{q_{3}-q_{4}}\right.$, 
donde $\mathbf{q}=\left(q_{3}, q_{4}\right)$ son las posiciones, $\mathbf{p}=\left(p_{3}, p_{4}\right)$ son los momentos conjugados y $\mathcal{M}=\operatorname{diag}(1+\epsilon, 1-\epsilon)$ es la matriz de masas reducidas.

Definimos el sistema Hamiltoniano asociado al problema de Sitnikov con $2+2$ cuerpos por $\mathcal{H}=\left(M, \omega, X_{H}\right)$, donde $M=T^{*} \mathcal{Q}$ es el espacio fase y corresponde al fibrado cotangente del espacio de configuración (2.9), $\omega=$ $\sum_{i} d p_{i} \wedge d q_{i}$ es la forma simpléctica canónica sobre $M$ y $X_{H}$ es el campo vectorial Hamiltoniano asociado a la función Hamiltoniana $H: M \rightarrow \mathbb{R}$ dada en (2.14).

El campo vectorial Hamiltoniano $X_{H}$ en coordenadas tiene la siguiente forma

$$
\begin{array}{ll}
\dot{q}_{3}=\frac{1}{1+\epsilon} p_{3}, & \dot{p}_{3}=-\frac{(1+\epsilon) q_{3}}{\left(q_{3}^{2}+r(t)^{2}\right)^{\frac{3}{2}}}-m \frac{1-\epsilon^{2}}{\left(q_{3}-q_{4}\right)^{2}}, \\
\dot{q}_{4}=\frac{1}{1-\epsilon} p_{4}, & \dot{p}_{4}=-\frac{(1-\epsilon) q_{4}}{\left(q_{4}^{2}+r(t)^{2}\right)^{\frac{3}{2}}}+m \frac{1-\epsilon^{2}}{\left(q_{3}-q_{4}\right)^{2}} .
\end{array}
$$

Es de vital importancia en el resto de este trabajo, notar que la función Hamiltoniana (2.14) puede escribirse como

$$
H=\underbrace{\left(\frac{1}{2(1+\epsilon)} p_{3}^{2}-\frac{1+\epsilon}{\sqrt{q_{3}^{2}+r(t)^{2}}}\right)}_{H_{3}}+\underbrace{\left(\frac{1}{2(1-\epsilon)} p_{4}^{2}-\frac{1-\epsilon}{\sqrt{q_{4}^{2}+r(t)^{2}}}\right)}_{H_{4}}+\underbrace{+m \frac{1-\epsilon^{2}}{q_{3}-q_{4}}}_{\hat{H}}
$$

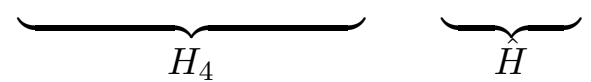

que corresponde a dos problemas de Sitnikov $H_{3}$ y $H_{4}$ acoplados por el término $\hat{H}$.

Nota 2.2. La simetría $\left(q_{3}, p_{3}, q_{4}, p_{4}, \epsilon\right) \leftrightarrow\left(q_{4}, p_{4}, q_{3}, p_{3},-\epsilon\right)$ permite restringir el análisis a valores no negativos del parámetro $\epsilon$.

La evolución de ambos secundarios esta restringida a la línea perpendicular que pasa por el centro de masas de los primarios. Esto significa que debemos considerar solamente una colisión unidimensional. Entonces, la transformación que regularizará el sistema es equivalente a la del problema colineal de Kepler.

Para realizar el estudio consideraremos los diferentes casos que separamos bajo los siguientes criterios: la excentricidad de las órbitas de los cuerpos primarios $e \in[0,1)$ nos permiten separar los casos siguientes:

- si $e=0$ tendremos el problema circular, que será integrable por cuadraturas,

- si $e \in(0,1)$ tendremos el problema elíptico que es un problema no integrable.

Por otro lado, el promedio de las masas infinitesimales $m \in\left[0, m_{0}\right)$ para $m_{0} \ll 1 / 2$ nos ofrece los casos siguientes:

- si $m=0$ no existirá interacción entre los cuerpos secundarios y el problema se estudia como un producto cartesiano de dos problemas de Sitnikov desacoplados. 
- si $0<m<m_{0}$ existirá una interacción entre los cuerpos secundarios y el sistema describirá una dinámica diferente al caso anterior.

Finalmente, el término $\epsilon \in\left[0, \epsilon_{0}\right)$ que relaciona las masas infinitesimales nos permite distinguir los siguientes casos:

- si $\epsilon=0$ los cuerpos secundarios tienen masas iguales y el flujo del problema se puede extender a un flujo completo.

- si $\epsilon \in\left(0, \epsilon_{0}\right)$ entonces los cuerpos secundarios tienen masas diferentes y el flujo no se podrá extender a un flujo completo y en general existirá un intercambio de momento.

Los elementos anteriores son independientes, por lo que se tendrán $2^{3}$ casos diferentes. Considerando notación binaria y asignando los valores binarios $\{0,1\}$ para cada condición como se muestra en el Cuadro 1. podemos ver que el caso con más restricciones corresponde al caso circular, sin interacción entre los secundarios y con masas iguales: 000. Por otro lado, el caso más general corresponde al caso elíptico, con interacción y masas distintas entre los secundarios: 111 .

\begin{tabular}{|r|c|c|c|}
\hline & ¿Es elíptico? & ¿Hay interacción? & ¿Tienen masas diferentes? \\
\hline Si & 1 & 1 & 1 \\
No & 0 & 0 & 0 \\
\hline
\end{tabular}

Cuadro 1. Clasificación de los diferentes casos en el problema de Sitnikov con $2+2$ cuerpos.

\subsection{Regularización DE COLISIONES.}

El sistema (2.15) tiene una singularidad cuando $q_{3}=q_{4}$, i.e. cuando los secundarios están en colisión. El procedimiento para trabajar con este tipo de singularidades ha sido estudiado por diversos matemáticos como Euler, Burrau, Thiele, Sundman, Levi-Civita, Birkhoff, Lamaître, Kustaanheimo y Stiefel, Conley, Easton, Waldvogel, entre otros y se conoce como regularización de singularidades. Existen muchas referencias que estudian detalladamente este proceso aplicado a problemas de la mecánica celeste, en particular Stiefel y Scheifel [96, Szebehely 97. y Hagihara [39] son de las más completas. Otra referencia más reciente es el libro editado por Celleti 18 mientras que Marchal 66 hace una clasificación sobre la regularización de los diferentes tipos de singularidades del problema de $N$ cuerpos.

Actualmente, el proceso de regularización de singularidades se utiliza en muchos otros campos de la física-matemática y cada campo tiene procesos adecuados para regularizar problemas particulares. En muchas teorías físicas, particularmente en teorías de campos de norma (gauge), las singularidades aparecen y están directamente relacionadas con los invariantes de la teoría. Este es el caso para las teorías de Yang-Mills, Chern-Simons, teoría 
de campos cuánticos, gravitación, entre otros 45. Este es un impedimento para establecer una definición de regularización de manera precisa.

Heurísticamente podemos decir que la regularización de una singularidad en un campo vectorial es una transformación local aplicada al campo vectorial que permite extender el flujo del nuevo campo vectorial al conjunto de singularidades. Fuera del conjunto de singularidades, el flujo de ambos campos vectoriales, el original y el transformado, es equivalente.

En la Sección [1.8 del Capítulo 1] se caracterizó a las singularidades del problema de los $N$-cuerpos que corresponden a escapes de algún cuerpo en tiempo finito. Dichas singularidades son esenciales y por lo tanto no son regularizables. Esto indica que las singularidades del problema de los $N$ cuerpos que se pueden regularizar corresponden a algunos tipos de colisiones donde las masas de los cuerpos cumplen ciertas características particulares. Un ejemplo es en el problema de 3-cuerpos, donde se sabe que la colisión triple no es regularizable para valores arbitrarios de las masas. Sin embargo, C. Simó 93 ha determinado un conjunto de valores para las masas de los tres cuerpos que permiten regularizar la colisión triple. Lo mismo sucede para otras colisiones multiples y colisiones binarias simultáneas que se pueden regularizar cuando las masas de los cuerpos que colisionan cumplen algunas restricciones. Un hecho importante es que las colisiones binarias aisladas siempre se pueden regularizar.

La clasificación de C. Marchal [66 establece dos tipos de regularizaciones para el problema de $N$-cuerpos: las analíticas, que utilizan un cambio de variables y un rescalamiento en el tiempo, y las topológicas, que utilizan bloques aislantes y el método de cirugía. Los bloques aislantes en una regularización topológica deben contener soluciones regulares alrededor de la solución $\mathbf{x}_{s}(t)$ que lleva a la singularidad $\left(\mathbf{x}_{s}, t_{s}\right)$. Si después de la singularidad las soluciones regulares permanecen acotadas entonces la solución singular se extiende por continuidad. En caso contrario se dice que la singularidad no es regularizable por el método de cirugía.

Las regularizaciones topológicas no dependen de la existencia de las regularizaciones analíticas y viceversa. En este sentido, decimos que son independientes unas de la otras. De hecho, algunas singularidades por colisión múltiple pueden ser regularizadas por el método de cirugía pero no por el método analítico. También existen singularidades que pueden ser regularizadas por ambos métodos y generalmente se llega a la misma extensión 71].

Para sistemas binarios podemos clasificar las colisiones en tres grupos:

1. colisiones entre dos cuerpos con masa finita,

2. colisiones entre dos cuerpos de masa infinitesimal,

3. colisiones entre un cuerpo de masa finita y un cuerpos de masa infinitesimal. 
Las singularidades debidas a colisiones binarias para sistemas rectilíneos no pueden regularizarse por el método de cirugía debido a que toda solución en cualquier vecindad va a colisión. Es decir, no podemos encontrar soluciones regulares que eviten la colisión para alguna vecindad de una solución singular. El problema de Sitnikov con $2+2$ cuerpos consiste en estudiar un subsistema rectilíneo, por lo que no será regularizable por el método de cirugía.

Por otro lado, las regularizaciones analíticas se realizan mediante un cambio de coordenadas local $\rho \in \operatorname{Dif}_{0}(M)$ y una función $g: M \rightarrow \mathbb{R}$ aplicadas al campo vectorial $X \in \mathfrak{X}(M)$ sobre la variedad $M$. En mecánica celeste, este proceso se puede aplicar directamente a la función Hamiltoniana $H: M \rightarrow \mathbb{R}$ que define el problema mecánico, de tal forma que la nueva función

$$
\check{\partial}(x ; h)=g(x) \cdot(H \circ \rho(x)-h), \quad x \in M, \quad h \in \mathbb{R}
$$

y el nuevo campo vectorial $X_{H}(\rho(x)), x \in M$, no tengan singularidades (localmente). El conjunto de nivel $\Sigma_{\{ð, h\}}:=\partial^{-1}(0 ; h) \subset M$ corresponderá a un nivel de energía fija $h \in \operatorname{Img}(H) \subset \mathbb{R}$ del sistema regularizado (que en general no será un conjunto invariante). Si además, el cambio de coordenadas $\rho: M \rightarrow M$ es un simplectomorfismo local, entonces se tendrá un sistema Hamiltoniano $\left(M, \omega, X_{ð}\right)$ equivalente fuera del conjunto de singularidades y por lo tanto los niveles de energía $\Sigma_{\{ð, h\}}$ serán conjuntos invariantes.

La función $g(x), x \in M$, actuará como un rescalamiento en el tiempo e identificará el tiempo real de las soluciones con un nuevo tiempo ficticio de la siguiente forma

$$
d t=g(x) d \tau, \quad x \in M .
$$

También son comunes la siguientes notaciones

$$
\frac{d t}{d \tau}=g(x) \quad \text { y } \quad \tau=\int_{t_{0}}^{t} \frac{d t}{g(x)} .
$$

Es importante notar que el nuevo sistema depende del tiempo ficticio $\tau$ y la función $g(x)$ puede determinar una obstrucción a la regularización de un sistema por el método analítico.

Este método fue denominado por Siegel y Moser como el truco de Poincaré [92, pág 35].

Definición 2.2. Decimos que una regularización analítica para un sistema Hamiltoniano $\left(M, \omega, X_{H}\right)$ es simpléctica, si el cambio de coordenadas $\rho: M \rightarrow M$ es un simplectomorfismo local.

Esto quiere decir que el sistema regularizado $\left(ð, X_{H}(\rho)\right)$, vuelve a ser un sistema Hamiltoniano de la forma $\left(M, \omega, X_{\check{\partial}}\right)$, pero que depende del nuevo tiempo ficticio $\tau$ y del valor de la energía $h$ como parámetro.

Definición 2.3. Sea $\left(M, \omega, X_{H}\right)$ un sistema Hamiltoniano tal que la función $H: M \rightarrow \mathbb{R}$ sólo tiene singularidades removibles. Decimos que 
una regularización analítica es global si la función $g: M \rightarrow \mathbb{R}$ cancela todas las singularidades de la función $H$; de otra forma diremos que es una regularización local.

Esta definición de regularización global únicamente concierne al problema que estamos considerando y no es global en el sentido estricto. Es decir que no elimina todas las singularidades de un problema de $N$ cuerpos si no sólo aquellas que se perciben en las ecuaciones. Siendo más específicos, consideremos una configuración de equilibrio relativo para $N$ cuerpos y consideremos otros $\nu$ cuerpos restringidos. Entonces el equilibrio relativo es un sistema integrable de dimensión $6 N$ y el sistema de $N+\nu$ cuerpos es un problema cuyo espacio fase tiene dimensión $6(N+\nu)$. Nuestra definición de regularización global únicamente considera una vecindad de la variedad de dimensión $6 \nu$ dentro del espacio de dimensión $6(N+\nu)$ correspondiente al equilibrio relativo.

\subsection{LA TRANSFORMACIÓN DE EULER.}

Se sabe que Euler 1 aplicaba la transformación $u=\sqrt{x}$ y el rescalamiento en el tiempo $d t=x d \tau$ para reducir el problema unidimensional de Kepler al oscilador armónico unidimensional cuando $h<0$, ver $\mathbf{7}$. Esta transformación puede reescribirse en coordenadas simplécticas naturales $(q, p) \in T^{*} \mathbb{R}$ como se verá a continuación.

Definición 2.4. Para $\mathbb{R}_{+}=\{x \in \mathbb{R} \mid x \geq 0\}$, la transformación de Euler $\xi: T^{*} \mathbb{R}_{+} \rightarrow T^{*} \mathbb{R}_{+}$es la aplicación dada por

$$
\xi:(Q, P) \mapsto\left(Q^{2} / 2, P / Q\right) \text {. }
$$

Llamaremos regularización de Euler a la pareja formada por la transformación de Euler y el rescalamiento en el tiempo dado por $d t=Q^{2} d \tau$.

Lema 2.3 (Jiménez-Pérez). La transformación de Euler $\xi: \mathcal{L} \rightarrow \mathcal{L}$ es un simplectomorfismo (local).

Demostración. El resultado se obtiene al comprobar de manera directa que la matriz Jacobiana

$$
d \xi=\left(\begin{array}{cc}
Q & -P / Q \\
0 & 1 / Q
\end{array}\right)
$$

es una matriz simpléctica.

Es posible considerar la inclusión de la transformación de Euler en el grupo de difeomorfimos locales $\operatorname{Dif}_{0}(M)$ sobre una variedad simpléctica $(M, \omega)$, que contenga un subespacio simpléctico lineal $\mathcal{L} \subset M$ de dimensión 2 , tal que $M \cong \mathcal{L} \oplus \mathcal{L}^{\omega}$.

\footnotetext{
${ }^{1}$ El autor no ha tenido acceso al artículo original Euler: Novi Comm. Acad. Sci. Imp. Petrop. 11, 144 (1767) que aparece como referencia en [7] y en otros artículos recientes.
} 
DEFINICIÓN 2.5. La inclusión canonica de la transformación de Euler en el grupo $\operatorname{Dif}_{0}(M)$ de una variedad simpléctica $(M, \omega)$ es el difeomorfismo

$$
i_{\xi}: \mathcal{L} \oplus \mathcal{L}^{\omega} \rightarrow M
$$

tal que

$$
\left.i_{\xi}\right|_{\mathcal{L}}=\xi \quad \text { and }\left.\quad i_{\xi}\right|_{\mathcal{L}^{\omega}}=i d_{\mathcal{L}^{\omega}} .
$$

Tenemos la equivalencia $\imath \circ \xi=i_{\xi} \circ \imath$, y por lo tanto el diagrama

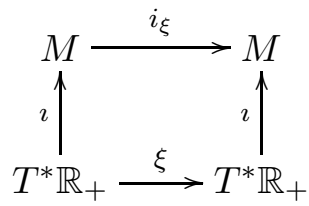

conmuta. En dicho diagrama se ha identificado $\imath\left(T^{*} \mathbb{R}_{+}\right) \cong \mathcal{L}$.

LEMA 2.4 (Jiménez-Pérez). La inclusión canónica de la transformación de Euler en el grupo $\operatorname{Dif}_{0}(M)$ es un simplectomorfismo local, es decir que $i_{\xi} \in S p(U, \omega)$ para $U \subset M$ abierto.

Demostración. Este resultado es una consecuencia inmediata de la suma directa de $\mathcal{L} \oplus \mathcal{L}^{\omega}$, entonces la matriz jacobiana de la diferencial de $i_{\xi}$ es exactamente

$$
d\left(i_{\xi}\right)=\left(\begin{array}{c|c}
(d \xi) & 0 \\
\hline 0 & I_{2(n-1)}
\end{array}\right)
$$

donde $d \xi \in \mathcal{M}_{2 \times 2}$ es la matriz jacobiana de la diferencial de la transformación de Euler y $I_{2(n-1)}$ es la matriz identidad en $\mathcal{M}_{2(n-1) \times 2(n-1)}$

Teorema 2.17 (Jiménez-Pérez). Toda solución particular del problema de $N$ cuerpos donde se tenga un único subsistema de dos cuerpos evolucionando de manera rectilínea (y tal que todas sus singularidades sean las colisiones binarias de ese subsistema) puede ser regularizada por una transformación simpléctica lineal y por la inclusión canónica de la transformación de Euler en el grupo simpléctico del espacio fase.

Demostración. Para el problema de $N$ cuerpos, la evolución de 2 cuerpos sobre una recta fija $\mathscr{L}$ del espacio físico $\mathbb{R}^{3}$, implica que el momento angular de dichos cuerpos es nulo y que el resto de los cuerpos evolucionan manteniendo su centro de masa sobre $\mathscr{L}$. Si consideramos el centro de masa en el origen, la ecuación de $\mathscr{L}$ se puede escribir de manera paramétrica mediante

$$
\mathscr{L}(\tau)=a \tau, \quad a \in \mathbb{R}^{3}, \quad \tau \in \mathbb{R}, .
$$

donde $a=\left(a_{1}, a_{2}, a_{3}\right)^{T}$ y $|a|=1$. Seleccionemos la matriz $B \in S O(3)$ que lleva el vector $a$ sobre el vector $e_{1}=(1,0,0)^{T}$ mediante un rotación alrededor del vector $a \times e_{1}$. De esta forma se cumple simultáneamente las condiciones

$$
B \cdot a=e_{1} \quad \mathrm{y} \quad B \cdot\left(a \times e_{1}\right)=a \times e_{1},
$$


que determinan de manera única a $B$.

Consideremos el espacio fase del problema de $N$ cuerpos como la variedad abierta con frontera $M=T^{*}\left(\mathbb{R}^{3 N} \backslash \Delta\right)$, de tal forma que los cuerpos que evolucionan sobre $\mathscr{L}$ tengan posiciones $q_{1}, q_{2} \in \mathbb{R}^{3}$ y momentos conjugados $p_{1}, p_{2} \in\left(\mathbb{R}^{3}\right)^{*}$. Aplicando la rotación $B$ al espacio fase mediante acción diagonal tenemos

$$
\mathbf{B} \cdot(\mathbf{q}, \mathbf{p})^{T}=\left(x_{1}, 0,0, x_{2}, 0,0, \ldots, \hat{q}_{N}, m_{1} \dot{x}_{1}, 0,0, m_{2} \dot{x}_{2}, 0,0, \ldots, \hat{p}_{N}\right)^{T}
$$

donde

$$
\begin{gathered}
\mathbf{B}=\operatorname{diag}(B, B, \ldots, B) \in M_{6 N \times 6 N}, \quad \mathbf{q}=\left(q_{1}, \ldots, q_{N}\right)^{T} \in \mathbb{R}^{3 N}, \\
\mathbf{p}=\left(p_{1}, \ldots, p_{N}\right)^{T} \in \mathbb{R}^{3 N}, \quad \hat{q}_{i}=B \cdot q_{i}, \quad \text { y } \quad \hat{p}_{i}=B \cdot p_{i}, \quad i=3, \ldots, N .
\end{gathered}
$$

Consideremos las matrices elementales $E_{i j} \in O(6 N)$ que intercambian los renglones $i$-ésimo y $j$-ésimo. Entonces tenemos que

$E_{(3 N+2,3 N+4)} E_{2,4} \mathbf{B} \cdot(\mathbf{q}, \mathbf{p})^{T}=\left(x_{1}, x_{2}, 0, \ldots, \hat{q}_{N}, m_{1} \dot{x}_{1}, m_{2} \dot{x}_{2}, 0, \ldots, \hat{p}_{N}\right)^{T}$. Consideramos la matriz $C \in G L(6 N)$ que lleva las componentes

$$
\begin{aligned}
\left(x_{1}, x_{2}\right) & \mapsto\left(x_{1}-x_{2}, \frac{m_{1} x_{1}+m_{2} x_{2}}{m_{1}+m_{2}}\right) \\
\left(m_{1} \dot{x}_{1}, m_{2} \dot{x}_{2}\right) & \mapsto\left(m_{1} m_{2}\left(\dot{x}_{1}-\dot{x}_{2}\right), m_{1} \dot{x}_{1}+m_{2} \dot{x}_{2}\right),
\end{aligned}
$$

que tiene la forma

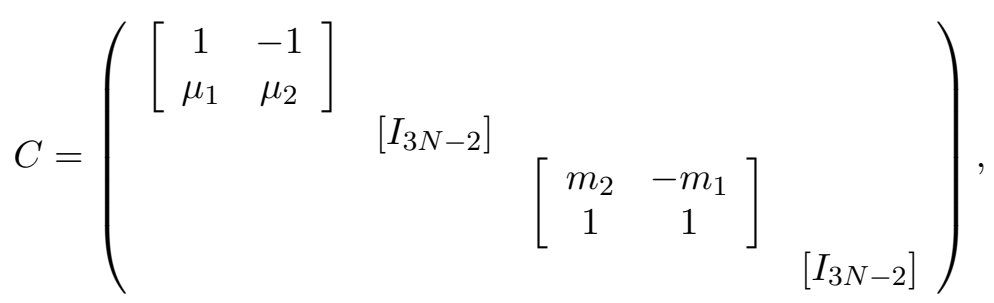

donde $I_{3 N-2}$ es la matriz identidad de dimensión $3 N-2 \times 3 N-2$ y las componentes restantes son cero.

Finalmente apliquemos la transformación elemental $E_{(2,3 N+1)}$ que llevará la componente colocada en la entrada $3 N+1$ a la segunda posición y visceversa.

Definamos la matriz $A \in G L(6 N)$ por

$$
A=E_{(2,3 N+1)} \cdot C \cdot E_{(3 N+2,3 N+4)} \cdot E_{(2,4)} \cdot \mathbf{B} .
$$

Un cálculo sencillo nos muestra que $J^{T} A J=J$ y por lo tanto $A \in$ $S p(6 N)$. Además

$$
A \cdot(\mathbf{q}, \mathbf{p})^{T}=\left(x_{1}-x_{2}, m_{1} m_{2}\left(\dot{x}_{1}-\dot{x}_{2}\right), 0, \ldots, \hat{p}_{N}\right)^{T} .
$$

Las dos primeras componentes definen un subespacio simpléctico $\left(\mathcal{L},\left.\omega\right|_{\mathcal{L}}\right)$ del espacio fase $(M, \omega)$. Existe otro subespacio simpléctico $\left(\mathcal{L}^{\omega},\left.\omega\right|_{\mathcal{L}^{\omega}}\right) \omega$ ortogonal tal que $M=\mathcal{L} \oplus \mathcal{L}^{\omega}$. El subespacio $\left(\mathcal{L},\left.\omega\right|_{\mathcal{L}}\right)$ contiene las singularidades del sistema cuando la primera componente vale cero y por hipótesis no existen otras singularidades. 
Ahora podemos aplicar la inclusión de la transformación de Euler tal que el espacio regularizado solo es afectado en el subespacio que contiene a las colisiones

$$
\mathcal{L} \oplus \mathcal{L}^{\omega} \stackrel{i_{\xi}}{\longrightarrow} \mathcal{R} \oplus \mathcal{L}^{\omega} .
$$

donde $\mathcal{R}$ es el subespacio simpléctico $\mathcal{L}$ bajo la regularización de Euler.

\subsection{REgUlarización DEL PROBLEMA CIRCULAR $2+2$ DE SitnikOV.}

Para eliminar las singularidades tanto en la función Hamiltoniana (2.14) como en el campo vectorial (2.15), aplicaremos una regularización simpléctica. De esta forma podemos extender analíticamente las ecuaciones del sistema al hiperplano $q_{3}=q_{4}$. Aplicamos la transformación $\rho: M \rightarrow M$ definida por

$$
\begin{array}{ll}
q_{3}=Q_{4}+(1-\epsilon) \frac{Q_{3}^{2}}{2}, & p_{3}=(1+\epsilon) P_{4}+\frac{P_{3}}{Q_{3}}, \\
q_{4}=Q_{4}-(1+\epsilon) \frac{Q_{3}^{2}}{2}, & p_{4}=(1-\epsilon) P_{4}-\frac{P_{3}}{Q_{3}}
\end{array}
$$

y el reescalamiento en el tiempo

$$
\frac{d t}{d \tau}=\left(1-\epsilon^{2}\right) Q_{3}^{2}
$$

Teorema 2.18 (Jiménez-Pérez). La transformación (2.27) es la composición de una transformación lineal simpléctica $A \in S p(M, \omega)$ y de la inclusión canónica de la transformación de Euler $i_{\xi}: T^{*} \mathbb{R}_{+} \rightarrow T^{*} \mathbb{R}_{+}$. Es decir

$$
\rho=i_{\xi} \circ A .
$$

La transformación lineal A lleva el espacio de configuración $\mathcal{Q}$ a coordenadas baricéntricas relativas.

Demostración. Para llevar el espacio de configuración a coordenadas baricéntricas relativas, es necesario aplicar la transformación lineal

$$
r_{3}=q_{3}-q_{4}, \quad r_{4}=\frac{1}{2}\left((1+\epsilon) q_{3}+(1-\epsilon) q_{4}\right)
$$

donde $r_{3}$ es la distancia entre los secundarios y $r_{4}$ es su baricentro relativo. Despejando $q_{3}$ y $q_{4}$ obtenemos

$$
q_{3}=r_{4}+(1-\epsilon) r_{3}, \quad q_{4}=r_{4}-(1+\epsilon) r_{3} .
$$

Esta transformación pertenece al grupo $\operatorname{Dif}(\mathcal{Q})$ y por lo tanto aplicando la Proposición 1.4 tenemos que su levantamiento cotangente producirá un simplectomorfismo lineal $A \in S p(M, \omega)$, tal que $(\mathbf{q}, \mathbf{p})^{T}=A \cdot(\mathbf{r}, \mathbf{s})^{T}$. Podemos utilizar funciones generatrices para obtener dicho simplectomorfismo 
de la siguiente forma: ya que conocemos tanto $\mathbf{p}$ como $\mathbf{r}$, entonces buscamos una función generatriz de tercer género $W(\mathbf{r}, \mathbf{p})$. Se debe verificar que $\mathbf{q}=\frac{\partial W}{\partial \mathbf{p}}(\mathbf{r}, \mathbf{p})$ y $\mathbf{s}=\frac{\partial W}{\partial \mathbf{r}}(\mathbf{r}, \mathbf{p})$, entonces

$$
W(\mathbf{r}, \mathbf{p})=p_{3}\left[r_{4}+(1-\epsilon) r_{3}\right]+p_{4}\left[r_{4}-(1+\epsilon) r_{3}\right],
$$

que producirá

$$
s_{3}=(1-\epsilon) p_{3}-(1+\epsilon) p_{4}, \quad s_{4}=p_{3}+p_{4} .
$$

Despejando $p_{3}$ y $p_{4}$ tenemos

$$
p_{3}=\frac{(1+\epsilon) s_{4}+s_{3}}{2}, \quad p_{4}=\frac{(1-\epsilon) s_{4}-s_{3}}{2} .
$$

Aplicando las transformaciones (2.30) y (2.31) concluimos que $A \in S p(M, \omega)$.

La composición de ambos nos dará

$$
\begin{aligned}
\left(Q_{3}, Q_{4}, P_{3}, P_{4}\right) & \stackrel{i_{\xi}}{\rightarrow}\left(\frac{1}{2} Q_{3}^{2}, Q_{4}, \frac{P_{3}}{Q_{3}}, P_{4}\right) \\
\stackrel{A}{\rightarrow} & \left(Q_{4}+\frac{\beta}{2} Q_{3}^{2}, Q_{4}-\frac{\alpha}{2} Q_{3}^{2}, \alpha P_{4}+\frac{P_{3}}{Q_{3}}, \beta P_{4}-\frac{P_{3}}{Q_{3}}\right),
\end{aligned}
$$

donde $\alpha=1+\epsilon$ y $\beta=1-\epsilon$. Este es el resultado buscado.

Como $\operatorname{Sp}(M, \omega)$ es un grupo bajo la composición, inmediatamente tenemos que la transformación (2.27) es también una transformación simpléctica sobre $(M, \omega)$ y por lo tanto, el diagrama

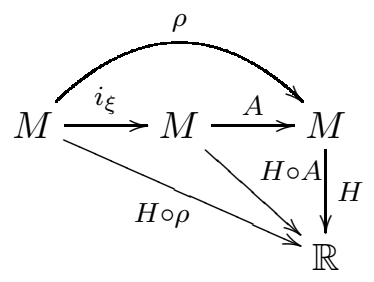

conmuta.

LEMA 2.5 (Jiménez-Pérez). La expresión

$$
W(\mathbf{Q}, \mathbf{p})=p_{3}\left(Q_{4}+(1-\epsilon) \frac{Q_{3}^{2}}{2}\right)+p_{4}\left(Q_{4}-(1+\epsilon) \frac{Q_{3}^{2}}{2}\right)
$$

determina una función generatriz para el simplectomorfismo (2.27).

Demostración. La función generatriz debe cumplir

$$
\mathbf{q}=\frac{\partial W}{\partial \mathbf{p}} \quad \text { y } \quad \mathbf{P}=\frac{\partial W}{\partial \mathbf{Q}} .
$$

Se tiene de manera inmediata que

$$
q_{3}=Q_{4}+(1-\epsilon) \frac{Q_{3}^{2}}{2} \quad \text { y } \quad q_{4}=Q_{4}-(1+\epsilon) \frac{Q_{3}^{2}}{2} .
$$

Derivando $W$ con respecto de $Q_{3}$ y $Q_{4}$ se tiene

$$
P_{3}=Q_{3}\left((1-\epsilon) p_{3}-(1+\epsilon) p_{4}\right), \quad P_{4}=p_{3}+p_{4} .
$$


Finalmente despejamos $p_{3}$ y $p_{4}$ para obtener la transformación (2.27).

Con esta regularización se obtiene una nueva función $\precsim(\mathbf{Q}, \mathbf{P} ; h)$ que depende del valor fijo de la energía $H=h$ como parámetro. Este proceso se realiza de la siguiente forma: primero se aplica el simplectomorfismo $H(\rho(\mathbf{Q}, \mathbf{P}))=H(\mathbf{q}, \mathbf{p})$ y la función en las nuevas variables $\bar{H}(\mathbf{Q}, \mathbf{P})=$ $H(\rho(\mathbf{Q}, \mathbf{P}))$ es nuevamente Hamiltoniana. Fijemos el valor de la función $h=H(\rho(\mathbf{Q}, \mathbf{P}))$ reacomodemos los términos y multipliquemos por el tiempo reescalado para obtener $\frac{d t}{d \tau}(H \circ \rho-h)=0$.

La función Hamiltoniana regularizada es

$$
\check{\partial}=\left(1-\epsilon^{2}\right) Q_{3}^{2}(H-h) \circ \rho
$$

esta función Hamiltoniana depende de $\epsilon, h$ como parámetros y es válida sólo en el nivel de energía $\precsim=0$ para cada $h$ fija. Para evitar cargar la notación escribiremos $\mathbf{z}=\left(Q_{3}, Q_{4}, P_{3}, P_{4}\right), \alpha=(1+\epsilon)$ y $\beta=(1-\epsilon)$ para tener

$$
\begin{aligned}
\partial= & \frac{1}{2}\left(\alpha \beta P_{4}^{2} Q_{3}^{2}+P_{3}^{2}\right)-2 \alpha^{2} \beta^{2} m \\
& -\alpha \beta Q_{3}^{2}\left[\frac{2 \alpha}{\sqrt{\left(2 Q_{4}+\beta Q_{3}^{2}\right)^{2}+1}}+\frac{2 \beta}{\sqrt{\left(2 Q_{4}-\alpha Q_{3}^{2}\right)^{2}+1}}+h\right] .
\end{aligned}
$$

Denotaremos por $\partial_{h}(\mathbf{z}, m ; \epsilon)=ð(\mathbf{z}, m ; \epsilon, h)$ a esta función.

DEFINICIÓN 2.6. Llamamos a la terna $\mathcal{R}_{h}=\left(M, \omega, X_{\varpi_{h}(\mathbf{z}, m)}\right)$ el sistema Hamiltoniano regularizado asociado a la función regularizada $\widehat{\partial}_{h}(\mathbf{z}, m)$, con campo Hamiltoniano $X_{\varlimsup_{h}(\mathbf{z}, m)}$ dado por

$$
\mathbf{Q}^{\prime}=\frac{\partial ð_{h}}{\partial \mathbf{P}}(\mathbf{z}, m) \quad \mathrm{y} \quad \mathbf{P}^{\prime}=-\frac{\partial ð_{h}}{\partial \mathbf{Q}}(\mathbf{z}, m),
$$

donde $^{\prime}=\frac{d}{d \tau}$ corresponde a la derivación con respecto del tiempo ficticio $\tau$.

Aunque la forma de la nueva función Hamiltoniana (2.33) es algo complicada, la ventaja es que esta función y el campo vectorial Hamiltoniano $X_{\varpi_{h}}$ pueden extenderse al conjunto de colisiones $\Delta$ de manera regular.

En las siguientes secciones, estudiaremos algunas reducciones del sistema Hamiltoniano bajo simetrías discretas. Para ello será necesario escribir de manera explícita el campo vectorial (2.34). El campo vectorial regularizado 


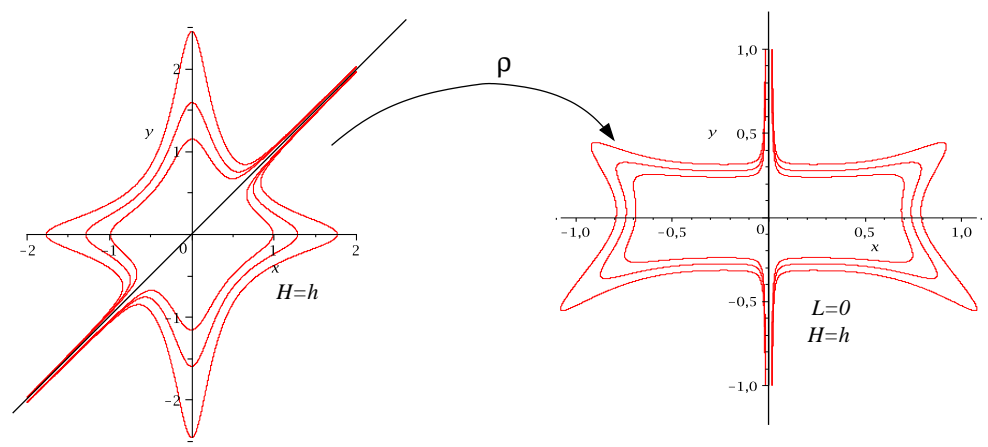

Figura 2.4. Curvas de velocidad cero para $H=h$ fija.

$X_{\varpi_{h}}$ escrito en coordenadas tiene la forma siguiente

$$
\begin{aligned}
Q_{3}^{\prime}= & P_{3}, \\
Q_{4}^{\prime}= & \alpha \beta Q_{3}^{2} P_{4}, \\
P_{3}^{\prime}= & -\alpha \beta Q_{3} P_{4}^{2}+4 \alpha \beta Q_{3}\left[\frac{\alpha\left(2\left(2 Q_{4}+Q_{3}^{2}\right) Q_{4}+1\right)}{\left[\left(2 Q_{4}+\beta Q_{3}^{2}\right)^{2}+1\right]^{\frac{3}{2}}}\right. \\
& \left.-\frac{\beta\left(2\left(2 Q_{4}-Q_{3}^{2}\right) Q_{4}+1\right)}{\left[\left(2 Q_{4}-\alpha Q_{3}^{2}\right)^{2}+1\right]^{\frac{3}{2}}}+h\right], \\
P_{4}^{\prime}= & -4 \alpha \beta Q_{3}^{2}\left[\frac{\alpha\left(2 Q_{4}+\beta Q_{3}^{2}\right)}{\left(\left(2 Q_{4}+\beta Q_{3}^{2}\right)^{2}+1\right)^{\frac{3}{2}}}\right. \\
& \left.+\frac{\beta\left(2 Q_{4}-\alpha Q_{3}^{2}\right)}{\left(\left(2 Q_{4}-\alpha Q_{3}^{2}\right)^{2}+1\right)^{\frac{3}{2}}}\right] .
\end{aligned}
$$

2.5.1. Simetrías DiscRetas. La función Hamiltoniana regularizada tiene un conjunto de simetrías que incluye algunas simetrías ficticias adquiridas por la regularización. Otro conjunto de simetrías puede extenderse si $\epsilon=0$ y finalmente existen las simetrías características de los sistemas mecánicos.

Definición 2.7. Para $M=T^{*}\left(\mathbb{R}^{n}\right), n \in \mathbb{N}$, el conjunto de simetrías lineales de un sistema Hamiltoniano $\left(M, \omega, X_{H}\right)$ está dado por el conjunto de transformaciones lineales $A \in \mathscr{L}(M)$ que preservan la función Hamiltoniana $H \circ A=H$, y el campo vectorial $X_{H}(A x)=X_{H}(x)$.

Definición 2.8. Consideremos un sistema Hamiltoniano $(M, \omega, H)$ donde $M=T^{*} \mathcal{Q}$ es el espacio fase. Al conjunto $\Gamma=\{(x, t) \in M \times \mathbb{R}\}$ se le conoce como el espacio fase extendido.

Las simetrías que deseamos considerar son básicamente las permutaciones e involuciones del espacio fase extendido $\Gamma \cong \mathbb{R}^{2 n+1}$. De manera precisa, sea $\beta=\left\{\beta_{1}, \ldots, \beta_{2 n}\right\}$ una base de $M$. Las simetrías que consideraremos serán las permutaciones $\sigma_{i}^{j}(\beta) \mapsto \operatorname{Aut}(M)$ tal que

$\sigma_{i}^{j}\left(\beta_{1}, \ldots, \beta_{i}, \ldots, \beta_{j}, \ldots, \beta_{2 n}\right)=\left(\beta_{1}, \ldots \beta_{j}, \ldots, \beta_{i}, \ldots, \beta_{2 n}\right), \quad 1 \leq i, j \leq n$ 
y las involuciones en $\Gamma$

$\varrho_{i}\left(\beta_{1}, \ldots \beta_{i}, \ldots, \beta_{2 n+1}\right)=\left(\beta_{1}, \ldots,-\beta_{i}, \ldots, \beta_{2 n+1}\right), \quad i \in\{1, \ldots, 2 n+1\}$.

En nuestro problema, podemos ver de manera inmediata que no existe ninguna simetría bajo permutaciones en los elementos de una base para $M$. Por lo que nos restará estudiar las simetrías que se obtienen bajo las involuciones de $\Gamma$

Consideremos las simetrías siguientes:

- $\mathcal{S}$ que corresponden a la reflexión con respecto de un subespacio simpléctico

- $\mathcal{S}_{1}\left(Q_{3}, Q_{4}, P_{3}, P_{4}, \tau\right)=\left(Q_{3},-Q_{4}, P_{3},-P_{4}, \pm \tau\right)$,

- $\mathcal{S}_{2}\left(Q_{3}, Q_{4}, P_{3}, P_{4}, \tau\right)=\left(-Q_{3}, Q_{4},-P_{3}, P_{4}, \pm \tau\right)$.

- $\mathcal{L}$ que corresponden a la reflexión con respecto de un subespacio Lagrangiano

- $\mathcal{L}_{1}\left(Q_{3}, Q_{4}, P_{3}, P_{4}, \tau\right)=\left(Q_{3}, Q_{4},-P_{3},-P_{4}, \pm \tau\right)$,

- $\mathcal{L}_{2}\left(Q_{3}, Q_{4}, P_{3}, P_{4}, \tau\right)=\left(-Q_{3},-Q_{4}, P_{3}, P_{4}, \pm \tau\right)$.

- I que corresponden a la reflexión con respecto de un subespacio isotrópico

- $\mathcal{I}_{1}\left(Q_{3}, Q_{4}, P_{3}, P_{4}, \tau\right)=\left(Q_{3},-Q_{4},-P_{3},-P_{4}, \pm \tau\right)$,

- $\mathcal{I}_{2}\left(Q_{3}, Q_{4}, P_{3}, P_{4}, \tau\right)=\left(-Q_{3}, Q_{4},-P_{3},-P_{4}, \pm \tau\right)$,

- $\mathcal{I}_{3}\left(Q_{3}, Q_{4}, P_{3}, P_{4}, \tau\right)=\left(-Q_{3},-Q_{4}, P_{3},-P_{4}, \pm \tau\right)$,

- $\mathcal{I}_{4}\left(Q_{3}, Q_{4}, P_{3}, P_{4}, \tau\right)=\left(-Q_{3},-Q_{4},-P_{3}, P_{4}, \pm \tau\right)$.

- $\mathcal{C}$ que corresponden a la reflexión con respecto de un subespacio co-isotrópico

- $\mathcal{C}_{1}\left(Q_{3}, Q_{4}, P_{3}, P_{4}, \tau\right)=\left(-Q_{3}, Q_{4}, P_{3}, P_{4}, \pm \tau\right)$,

- $\mathcal{C}_{2}\left(Q_{3}, Q_{4}, P_{3}, P_{4}, \tau\right)=\left(Q_{3},-Q_{4}, P_{3}, P_{4}, \pm \tau\right)$,

- $\mathcal{C}_{3}\left(Q_{3}, Q_{4}, P_{3}, P_{4}, \tau\right)=\left(Q_{3}, Q_{4},-P_{3}, P_{4}, \pm \tau\right)$,

- $\mathcal{C}_{4}\left(Q_{3}, Q_{4}, P_{3}, P_{4}, \tau\right)=\left(Q_{3}, Q_{4}, P_{3},-P_{4}, \pm \tau\right)$.

Como la regularización agrega una simetría ficticia para $Q_{3}^{2}=q_{3}-q_{4} \mathrm{y}$ por hipótesis $\mathcal{Q}$ sólo considera los casos $q_{3}>q_{4}$, entonces descartamos las simetrías $\mathcal{S}_{2}, \mathcal{L}_{2}, \mathcal{I}_{2}, \mathcal{I}_{3}, \mathcal{I}_{4}$ y $\mathcal{C}_{1}$.

La simetría $\mathcal{L}_{1}$ refleja la reversibilidad con respecto del tiempo ficticio $\tau$

$$
\left(Q_{3}, Q_{4}, P_{3}, P_{4}, \tau\right) \mapsto\left(Q_{3}, Q_{4},-P_{3},-P_{4},-\tau\right),
$$

que es una propiedad genérica de los sistemas mecánicos.

Mientras que si deseamos cambiar $Q_{4} \leftrightarrow-Q_{4}$, se invierten los valores de $P_{3}^{\prime}$ y $P_{4}^{\prime}$ pero no los de $P_{3}$ y $P_{4}$, entonces la simetría $\mathcal{I}_{2}$

$$
\left(Q_{3}, Q_{4}, P_{3}, P_{4}, \tau\right) \mapsto\left(Q_{3},-Q_{4},-P_{3},-P_{4},-\tau\right)
$$

invierte los valores de las masas relativas $(1-\epsilon) \leftrightarrow(1+\epsilon)$.

Podemos enunciar el siguiente resultado:

Proposición 2.13 (Jiménez-Pérez). El sistema Hamiltoniano regularizado $\left(M, \omega, X_{\check{\partial}_{h}}\right)$ es simétrico con respecto del hiperplano $Q_{4}=0$ si y sólo si $\epsilon=0$. Además, este plano es invariante bajo el flujo del campo Hamiltoniano regularizado $X_{\varpi_{h}}$. 
Demostración. La simetría se obtiene de manera trivial a partir de las ecuaciones (2.33) y (2.35) que implican la condición $\alpha=\beta$ y esto se cumple si y sólo si $\epsilon=0$.

Ahora si $Q_{4} \equiv 0$ para todo tiempo $\tau$, entonces de la cuarta ecuación en (2.35) se tiene que $P_{4}^{\prime}=0$ y por lo tanto $P_{4}=$ constante. Además, $Q_{4}^{\prime} \equiv 0$, pero $\alpha \neq 0, \beta \neq 0$ y $Q_{3} \neq 0$ no es idénticamente cero. Entonces $P_{4}=0$ y tenemos el sistema reducido

$$
\begin{aligned}
Q_{3}^{\prime} & =P_{3}, \\
P_{3}^{\prime} & =4 Q_{3}\left(\frac{2}{\left(Q_{3}^{4}+1\right)^{\frac{3}{2}}}+h\right), \\
Q_{4}^{\prime} & =0 \\
P_{4}^{\prime} & =0
\end{aligned}
$$

lo que indica que $Q_{4}=0$ es un plano invariante bajo el flujo del campo vectorial (2.35).

Los sistemas Hamiltonianos $\left(M, \omega, X_{H}\right)$ que poseen planos de simetría invariantes bajo el flujo $\varphi_{t}^{H}$, permiten reducir el sistema al plano invariante. Como el plano invariante $Q_{4}=P_{4}=0$ implica que el centro de masas se encuentra en el origen, entonces la evolución de los secundarios es simétrica con respecto del plano donde evolucionan los primarios.

Definición 2.9. Decimos que una solución $\sigma(t)$ del problema de Sitnikov con $2+2$ cuerpos tiene condiciones iniciales simétricas, si para el tiempo inicial $t=t_{0}$ se tiene que $q_{3}\left(t_{0}\right)=-q_{4}\left(t_{0}\right)$ y $p_{3}\left(t_{0}\right)=-p_{4}\left(t_{0}\right)$.

Proposición 2.14 (Jiménez-Pérez). El problema circular de Sitnikov de $2+2$ cuerpos con masas de los secundarios iguales $(\epsilon=0)$ y condiciones iniciales simétricas es integrable por cuadraturas.

Demostración. Esta es una consecuencia inmediata de la Proposición 2.13. ya que tenemos un problema Hamiltoniano autónomo con un grado de libertad. Entonces

$$
\tilde{\mathrm{\partial}}_{h}\left(Q_{3}, P_{3} ; m\right)=\frac{1}{2} P_{3}^{2}-2 m-Q_{3}^{2}\left(\frac{2}{\sqrt{Q_{3}^{4}+1}}+h\right)
$$

es una primera integral con $\tilde{\mathrm{d}}_{h}\left(Q_{3}, P_{3} ; m\right)=0$. A partir del campo vectorial (2.38) obtenemos $\frac{d Q_{3}}{d \tau}=P_{3}$. Sustituyendo en (2.39) y despejando llegamos a

$$
\tau-\tau_{0}=\int_{0}^{q} \frac{d Q_{3}}{\sqrt{2}\left(2 m+Q_{3}^{2}\left(\frac{2}{\sqrt{Q_{3}^{4}+1}}+h\right)\right)^{\frac{1}{2}}} .
$$

El sistema Hamiltoniano reducido $\left(N, \omega, X_{\tilde{\tilde{d}}_{h}(x ; m)}\right), x \in N$, está definido sobre la subvariedad simpléctica bidimensional $N=T^{*} \mathbb{R}_{+}$con campo 
vectorial $X_{\tilde{\tilde{\partial}}_{h}(x ; m)}$ dado por

$$
\begin{aligned}
& Q_{3}^{\prime}=P_{3}, \\
& P_{3}^{\prime}=4 Q_{3}\left(\frac{2}{\left(Q_{3}^{4}+1\right)^{\frac{3}{2}}}+h\right) .
\end{aligned}
$$

Mediante la regularización, este sistema Hamiltoniano también es regular en el conjunto reducido de singularidades $\tilde{\Delta}=\left\{Q_{3}=0\right\} \subset \Delta$. Por lo tanto, la función y el campo vectorial se han extendido a $\tilde{N}=N \cup \tilde{\Delta}$.

Considerando el rebote elástico en las colisiones, sólo el semiplano derecho de la Figura 2.5 es válido y corresponde precisamente a $\tilde{N}$. Sin embargo como $\epsilon=0$ es posible continuar las soluciones a través de la recta $Q_{3}=0$ mediante la simetría $\left(Q_{3}, P_{3}, \tau\right) \leftrightarrow\left(-Q_{3}, P_{3}, \tau\right)$ y obtener las curvas de nivel del espacio fase regularizado reducido en todo el plano $\left(Q_{3}, P_{3}\right)$ como se muestra en dicha figura.

En el capítulo siguiente, veremos que para identificar ambos semiespacios es necesario que $\epsilon=0$.

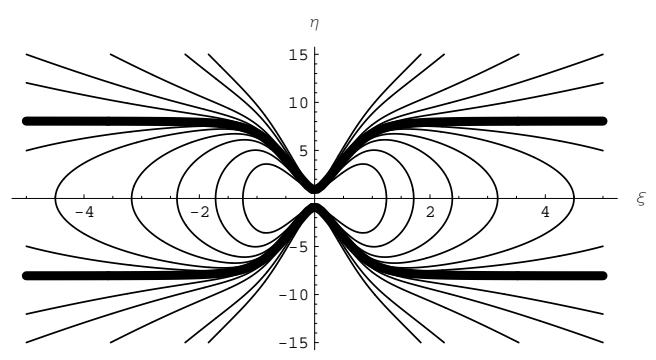

Figura 2.5. Curvas de nivel del problema circular de Sitnikov de $2+2$ cuerpos con condiciones iniciales simétricas.

Proposición 2.15 (Jiménez-Pérez). El problema circular de Sitnikov con $2+2$ cuerpos con condiciones iniciales simétricas tiene la siguiente dinámica:

- Si $h<0$ se tienen órbitas cerradas periódicas, donde los secundarios colisionan en el origen.

- Si $h=0$ se tiene una órbita parabólica donde los secundarios vienen de infinito con velocidad nula, pasan por una colisión en el origen y se escapan a infinito en sentidos opuestos con velocidad asintótica a 0 .

- Si $h>0$ se tienen órbitas hiperbólicas donde los secundarios vienen de infinito con velocidad positiva, pasan por una colisión en el origen y se escapan a infinito en sentidos opuestos con velocidad positiva.

Demostración. Un sistema Hamiltoniano con un grado de libertad es integrable y por lo tanto el grupo de Lie que actúa sobre el espacio fase 
solo puede ser $G=\left(\mathbb{S}^{1}, \cdot\right)$ o $G=(\mathbb{R},+)$. Para el caso en que $h<0$ se tiene la acción Hamiltoniana de $\mathbb{S}^{1}$ sobre el abierto $U \subset \mathbb{R}^{2}$ que contiene al origen del espacio fase regularizado. Aquí se tienen órbitas periódicas donde los cuerpos secundarios tienen posiciones simétricas con respecto al origen: $q_{3}=-q_{4}$ y con momentos $p_{3}=-p_{4}$. Por lo tanto un periodo consiste en que los dos cuerpos salen del origen (expulsión) con velocidad infinita y en sentidos opuestos, de forma que su evolución siempre es simétrica con respecto del origen. En un tiempo finito $\tau / 2$ llegan a una distancia máxima finita y regresan al origen al tiempo $\tau$ donde se producirá la colisión.

Para el caso $h \geq 0$ se tiene el grupo de Lie $(G=\mathbb{R},+)$ actuando sobre $\mathbb{R}^{2} \backslash \bar{U}$ donde $\bar{U}=U \cup \partial U$. Aquí se tienen órbitas que no son periódicas y que mediante la regularizaciíon y la identificación $\left(-q_{3},-q_{4}\right) \leftrightarrow\left(q_{3}, q_{4}\right)$, podemos extender las soluciones para todo $\tau \in \mathbb{R}$. Si $\tau \rightarrow \pm \infty$ tenemos que $q_{3} \rightarrow \pm \infty$ y dada la simetría $q_{3}=-q_{4}$ tenemos la dinámica siguiente: los cuerpos inician su evolución en infinito de lados opuestos del plano de evolución de los primarios. Se acercan al origen de manera simétrica para colisionar en el origen con rebote elástico y finalizar con un escape simultáneo a infinito en sentidos opuestos. La velocidad de escape (e inicio de evolución) se obtiene con el límite $\operatorname{lím}_{q_{i} \rightarrow \infty} \dot{q}_{i}$ donde

$$
\dot{q}_{i}= \pm \sqrt{h+\frac{2}{\sqrt{q_{i}^{2}+1 / 4}}+\frac{m}{2 q_{i}}}
$$

Obtenemos el límite

$$
\lim _{q_{i} \rightarrow \infty} \dot{q}_{i}= \pm \sqrt{h}
$$

de donde se concluye que los cuerpos secundarios se escapan con velocidad cero si y sólo si $h=0$. Por lo tanto para $h=0$ se tiene una órbita parabólica para cada secundario y si $h>0$ se tienen órbitas hiperbólicas para cada secundario.

Nota 2.3. En el problema simétrico, las ecuaciones permiten obtener dos órbitas parabólicas si $h=0$ y dos órbitas hiperbólicas si $h>0$ una que corresponde a $q_{3} \rightarrow \infty$ y la otra a $q_{3} \rightarrow-\infty$. Por hipótesis $q_{3}>q_{4}$ así que podemos considerar solamente el valor positivo de la raíz. 


\section{CAPÍTULO 3}

\section{ESTUDIO DEL CASO RESTRINGIDO}

En este capítulo se realiza un estudio detallado del problema circular de Sitnikov con $2+2$ cuerpos cuando los cuerpos secundarios no interactúan entre ellos. Éstos equivalen a los casos 000 y 001 del Cuadro 1 y corresponde a los valores $m=0$ y $r(t)=1 / 2$ en la función (2.14) y las ecuaciones (2.15).

Iniciamos el capítulo obteniendo el caso restringido $m \rightarrow 0$. El sistema Hamiltoniano se desacoplará y las ecuaciones de movimiento pueden integrarse de manera independiente, lo que nos dará, efectivamente, el caso integrable del problema de Sitnikov de $2+2$ cuerpos. Posteriormente, obtenemos las soluciones explícitamente y las coordenadas acción-ángulo para proseguir con un estudio de las superficies de energía constante y su foliación por subvariedades Lagrangianas. Finalizamos el capítulo caracterizando las órbitas periódicas del problema cuando las masas infinitesimales de los secundarios son iguales, es decir, cuando $\epsilon=0$.

\subsection{El PROBLEMA CIRCULAR RESTRINGIDO DE SitnikOV CON $2+2$ CUERPos.}

En el Capítulo 2 se estudió la regularización del sistema Hamiltoniano singular $\left(M, \omega, X_{H}\right)$ donde

$$
H=\left(\frac{1}{2(1+\epsilon)} p_{3}^{2}-\frac{1+\epsilon}{\sqrt{q_{3}^{2}+1 / 4}}\right)+\left(\frac{1}{2(1-\epsilon)} p_{4}^{2}-\frac{1-\epsilon}{\sqrt{q_{4}^{2}+1 / 4}}\right)+m \frac{1-\epsilon^{2}}{q_{3}-q_{4}},
$$

y que tiene como función regularizada a $\varlimsup_{h}(\mathbf{z}, m ; \epsilon)$ definida por la expresión (2.33).

El sistema Hamiltoniano regularizado $\hat{\mathrm{d}}_{h}=\left(M, \omega, X_{\varlimsup_{h}(\mathbf{z}, m ; \epsilon)}\right)$ es equivalente al sistema original sobre la variedad abierta $M$. Sin embargo, para estudiar el caso sin interacción entre los secundarios debemos encontrar el 
límite

$$
\lim _{m \rightarrow 0} \partial_{h}(\mathbf{z}, m ; \epsilon)=\varlimsup_{h}(\mathbf{z}, 0 ; \epsilon),
$$

que eliminará el término $2\left(1-\epsilon^{2}\right)^{2} m=2 \alpha^{2} \beta^{2} m$ en (2.33).

Como la función Hamiltoniana regularizada cumple

$$
\widetilde{\partial}_{h}(\mathbf{z}, 0 ; \epsilon)=g \cdot\left(\left.H\right|_{m=0}-h\right) \circ(\rho)(\mathbf{z})=0,
$$

para todo tiempo, y $g(\mathbf{z})$ no es idénticamente cero, entonces

$$
\left(\left.H\right|_{m=0}-h\right) \circ(\rho)(\mathbf{z})=0 .
$$

Finalmente, aplicamos la transformación inversa $\rho^{-1} \in S p(M)$ y obtenemos

$$
H=\left(\frac{1}{2(1+\epsilon)} p_{3}^{2}-\frac{1+\epsilon}{\sqrt{q_{3}^{2}+1 / 4}}\right)+\left(\frac{1}{2(1-\epsilon)} p_{4}^{2}-\frac{1-\epsilon}{\sqrt{q_{4}^{2}+1 / 4}}\right) .
$$

Podemos escribir la función Hamiltoniana del problema circular restringido como

$$
H=H_{3}+H_{4},
$$

donde $H_{i}$ con $i=3$, 4 son las funciones Hamiltonianas para dos problemas de Sitnikov independientes (desacoplados). Sin embargo, en estas ecuaciones no se percibe la interacción de los secundarios al momento de la colisión.

En las siguientes secciones haremos un estudio de los niveles de energía fijos y del comportamiento de las soluciones cuando se pasa a través de colisión. Con esto veremos que aunque se tiene un sistema integrable, las colisiones pueden dar paso a dinámica muy diversa.

\subsection{SOLUCiÓN ANALÍTICA DEL CASO CIRCULAR RESTRINGIDO.}

Para poder hacer un estudio de las soluciones del problema completo, primero haremos un estudio extenso del caso integrable. Utilizando la expresión para el caso circular restringido podemos ver que se tiene el caso integrable para $m_{3}=m_{4}=0$, lo que corresponde a dos problemas circulares de Sitnikov desacoplados (de ahí el nombre de doble Sitnikov). El caso integrable fue estudiado por Pavanini en 1907 [82] y por MacMillan en 1910 65. quienes expresaron la solución en términos de funciones elípticas de Weierstrass y Jacobi respectivamente. A partir de este momento y en lo que resta de este capítulo se asume que $\epsilon=0$.

Nota 3.4. Escribiremos a lo largo del trabajo y por abuso de notación que las energías relativas de cada secundario son

$$
h_{3}=\frac{\tilde{h}_{3}}{1+\epsilon} \quad y \quad h_{4}=\frac{\tilde{h}_{4}}{1-\epsilon} .
$$

Esta relación tiene impacto en los tres casos siguientes:

- en los módulos de las funciones e integrales elípticas $k_{3}, k_{4}$, 
- en la imagen de la aplicación momento $\mu$ y

- en el cálculo del tiempo de colisión para la continuación de órbitas simétricas.

En cada uno de estos casos se establece un recodatorio para que no haya riesgo de confusión.

Iniciemos con un resultado adicional que no se encuentra en la literatura.

TeOrema 3.19 (Jiménez-Pérez). Las coordenadas acción-ángulo para el problema circular restringido de Sitnikov con $2+2$ cuerpos tienen la forma

$$
\begin{aligned}
J\left(h_{i}\right) & =\frac{\sqrt{2}}{\pi}\left(2 E\left(k_{i}\right)-K\left(k_{i}\right)-\Pi\left(2 k_{i}^{2}, k_{i}\right)\right), \\
\theta_{i}\left(t ; h_{i}\right) & =\frac{1}{\Omega_{i}} t\left(\nu_{i}, k_{i}\right)+\theta_{0, i},
\end{aligned}
$$

donde $\Omega_{i}=\frac{\sqrt{2}}{4 \pi\left(1-2 k_{i}^{2}\right)}\left(2 E\left(k_{i}\right)-K\left(k_{i}\right)+\Pi\left(2 k_{i}^{2}, k_{i}\right)\right)$ es el tiempo de retorno de los secundarios y $\theta_{0, i}, i=3,4$, son constantes determinadas por las condiciones iniciales. $K\left(k_{i}\right), E\left(k_{i}\right)$ y $\Pi\left(2 k_{i}^{2}, k_{i}\right), i=3,4$, son las integrales elípticas completas de primera, segunda y tercera especie respectivamente, donde los módulos están dados por

$$
k_{3}=\frac{\sqrt{2+\frac{h_{3}}{1+\epsilon}}}{2}, \quad \text { y } \quad k_{4}=\frac{\sqrt{2+\frac{h_{4}}{1-\epsilon}}}{2} .
$$

Demostración. Como tenemos un sistema Hamiltoniano separable entonces cada nivel fijo de energía $H=h$ se puede escribir como $h=h_{3}+h_{4}$ con $h_{3}, h_{4}$ fijos. La acción está definida por la integral de la 1-forma de Liouville $J_{i}\left(h_{i}\right)=\frac{1}{2 \pi} \oint p_{i} d q_{i}, i=3,4$, sobre un periodo completo. Debido a que cada solución periódica $\gamma_{h_{i}}$ es simétrica en $p_{i}$ y $q_{i}$, podemos integrar sobre un cuarto del periodo y multiplicar dicho resultado por cuatro

$$
J_{i}\left(h_{i}\right)=\frac{2 \sqrt{2}}{\pi} \oint_{0}^{q_{\text {max }}} \sqrt{h_{i}+\frac{1}{\sqrt{q_{i}^{2}+\frac{1}{4}}}} d q_{i},
$$

donde $q_{i \max }$ se obtiene cuando $p_{i}=0$, entonces $q_{i \max }=\sqrt{\frac{1}{\left(-h_{i}\right)^{2}}-\frac{1}{4}}$.

Construimos un cambio de variables adecuado $\left(q_{i}, p_{i}\right) \mapsto\left(y_{i}, s_{i}\right)$ que "normalice" la integral, es decir, tal que las siguientes condiciones se cumplan:

a) La función Hamiltoniana tenga la forma $\hat{h}=y_{i}^{2} / 2+a s_{i}^{2}$, de aquí se tendrá que

$$
-\frac{1}{\sqrt{q_{i}^{2}+\frac{1}{4}}}=a s_{i}^{2}+b .
$$


b) Para $q_{i}=0$ debemos tener que $s_{i}=0$, y por lo tanto

$$
-\left.\frac{1}{\sqrt{q_{i}^{2}+\frac{1}{4}}}\right|_{q_{i}=0}=-2
$$

de donde $b=-2$.

c) Para $q_{i}=q_{i_{\max }}\left(\mathrm{y} p_{i}=0\right)$ se requiere que $s_{i}=1$,

$$
\begin{aligned}
-\left.\frac{1}{\sqrt{q_{i}^{2}+\frac{1}{4}}}\right|_{q_{i}=\sqrt{\frac{1}{\left(-h_{i}\right)^{2}}-\frac{1}{4}}} & =h_{i}, \\
h_{i} & =\left.\left(a s_{i}^{2}-2\right)\right|_{s_{i}=1},
\end{aligned}
$$

de donde $a=2+h_{i}$.

El cambio de variables

$$
\left(-\frac{1}{\sqrt{q_{i}^{2}+1 / 4}}, p_{i}\right)=\left(\left(2+h_{i}\right) s_{i}^{2}-2, y_{i}\right)
$$

transforma el integrando (3.6) en $\sqrt{h_{i}+\frac{1}{\sqrt{q_{i}^{2}+1 / 4}}}=\sqrt{2+h_{i}} \sqrt{1-s_{i}^{2}}$. Escribamos $k_{i}=\sqrt{2+h_{i}} / 2$ y resolvamos (3.7) para $q_{i}$, después calculemos $d q_{i}$ para obtener

$$
\sqrt{h_{i}+\frac{1}{\sqrt{q_{i}^{2}+1 / 4}}} d q_{i}=2 k_{i}^{2} \frac{\sqrt{1-s_{i}^{2}}}{\sqrt{1-k_{i}^{2} s_{i}^{2}}\left(1-2 k_{i}^{2} s_{i}^{2}\right)^{2}} d s_{i} .
$$

La integral (3.6) toma la forma

$$
J_{i}\left(h_{i}\right)=\frac{4 \sqrt{2}}{\pi} k_{i}^{2} \oint_{0}^{1} \frac{\sqrt{1-s_{i}^{2}}}{\sqrt{1-k_{i}^{2} s_{i}^{2}}\left(1-2 k_{i}^{2} s_{i}^{2}\right)^{2}} d s_{i} .
$$

Esta es una integral elíptica general completa. Es posible escribir toda integral elíptica general en términos de funciones algebraicas racionales de la variable independiente y de integrales elípticas de primera, segunda y tercera especie [40. Procedamos a escribir la integral (3.9) de esa forma. Primero integremos por partes considerando que $p_{i} d q_{i}=p_{i} q_{i}-q_{i} d p_{i}$ y donde $p_{i}=\sqrt{1-s_{i}^{2}}$ y $q_{i}=\frac{s_{i} \sqrt{1-k_{i}^{2} s_{i}^{2}}}{1-2 k_{i}^{2} s_{i}^{2}}$. Con ello tenemos

$$
\int \frac{\sqrt{1-s_{i}^{2}} d s_{i}}{\sqrt{1-k_{i}^{2} s_{i}^{2}}\left(1-2 k_{i}^{2} s_{i}^{2}\right)^{2}}=\frac{s_{i} \sqrt{1-s_{i}^{2}} \sqrt{1-k_{i}^{2} s_{i}^{2}}}{1-2 k_{i}^{2} s_{i}^{2}}+\int \frac{s_{i}^{2} \sqrt{1-k_{i}^{2} s_{i}^{2}} d s_{i}}{\sqrt{1-s_{i}^{2}}\left(1-2 k_{i}^{2} s_{i}^{2}\right)} .
$$


Reescribamos la última integral de la siguiente forma

$$
\frac{1}{2 k_{i}^{2}} \int \frac{2 k_{i}^{2} s_{i}^{2} \sqrt{1-k_{i}^{2} s_{i}^{2}} d s_{i}}{\sqrt{1-s_{i}^{2}}\left(1-2 k_{i}^{2} s_{i}^{2}\right)}=-\frac{1}{2 k^{2}} E\left(s_{i}, k_{i}\right)+\frac{1}{2 k_{i}^{2}} \int \frac{\sqrt{1-k_{i}^{2} s_{i}^{2}} d s_{i}}{\sqrt{1-s_{i}^{2}}\left(1-2 k_{i}^{2} s_{i}^{2}\right)},
$$

y nuevamente la última integral se reescribirá como

$$
\frac{1}{4 k_{i}^{2}} \int \frac{\left[1+\left(1-2 k_{i}^{2} s_{i}^{2}\right)\right] d s_{i}}{\sqrt{1-k_{i}^{2} s_{i}^{2}} \sqrt{1-s_{i}^{2}}\left(1-2 k_{i}^{2} s_{i}^{2}\right)}=\frac{1}{4 k_{i}^{2}} F\left(s_{i}, k_{i}\right)+\frac{1}{4 k_{i}^{2}} \Pi\left(2 k_{i}^{2}, s_{i}, k_{i}\right) \text {. }
$$

Colocando todos los términos juntos tenemos

$$
\begin{aligned}
& \int \frac{\sqrt{1-s_{i}^{2}} d s_{i}}{\sqrt{1-k_{i}^{2} s_{i}^{2}}\left(1-2 k_{i}^{2} s_{i}^{2}\right)^{2}} d s_{i}= \frac{s_{i} \sqrt{1-s_{i}^{2}} \sqrt{1-k_{i}^{2} s_{i}^{2}}}{1-2 k_{i}^{2} s_{i}^{2}}- \\
& \frac{1}{4 k_{i}^{2}}\left(2 E\left(s_{i}, k_{i}\right)-\Pi\left(2 k_{i}^{2}, s_{i}, k_{i}\right)-F\left(s_{i}, k_{i}\right)\right) .
\end{aligned}
$$

Finalmente, evaluando en los límites de integración, obtenemos (3.4).

Teorema 3.20 (Jiménez-Pérez 2009, Corbera y Llibre 2002). Las soluciones del problema circular restringido de Sitnikov con $2+2$ cuerpos se expresan en términos de las funciones elípticas de Jacobi de la forma

$$
\phi(t)=\left(k_{3} \frac{s_{3} d_{3}}{1-2 k_{3}^{2} s_{3}^{2}}, 2 \sqrt{2} k_{3} c_{3}, k_{4} \frac{s_{4} d_{4}}{1-2 k_{4}^{2} s_{4}^{2}}, 2 \sqrt{2} k_{4} c_{4}\right),
$$

donde $s_{i}=\operatorname{sn}\left(\nu_{i}, k_{i}\right), c_{i}=\operatorname{cn}\left(\nu_{i}, k_{i}\right) y d_{i}=\operatorname{dn}\left(\nu_{i}, k_{i}\right)$ son las funciones elípticas de Jacobi, $k_{3}=\frac{\sqrt{2+\frac{h_{3}}{1+\epsilon}}}{2}, k_{4}=\frac{\sqrt{2+\frac{h_{4}}{1-\epsilon}}}{2} y \nu_{i}=\nu_{i}\left(t, k_{i}\right)$ se obtiene al invertir la función

$$
t\left(\nu_{i}, k_{i}\right)=\frac{1}{2 \sqrt{2}} \int_{0}^{\nu_{i}} \frac{d \eta}{\left(1-2 k_{i}^{2} \operatorname{sn}^{2}\left(\eta, k_{i}\right)\right)^{2}},
$$

para $i=3,4$.

Demostración. Como la solución de un sistema Hamiltoniano Newtoniano con un grado de libertad que tiene la forma $H=p^{2} / 2-V(q)$ es $t+t_{0}=\int \frac{d q}{p}$ con $p=\sqrt{2(h+V(q))}$, podemos usar el cambio de variables (3.7) en la diferencial

$$
d t=\frac{d q_{i}}{\sqrt{h_{i}+\frac{1}{\sqrt{q_{i}^{2}+1 / 4}}}}=\frac{1}{2 \sqrt{2}} \frac{d s_{i}}{\sqrt{1-s_{i}^{2}} \sqrt{1-k_{i}^{2} s_{i}^{2}}\left(1-2 k_{i}^{2} s_{i}^{2}\right)^{2}},
$$

reescalar el tiempo por $\frac{d s_{i}}{d \nu} d \nu$ para obtener

$$
\frac{d t}{d \nu}=\frac{1}{2 \sqrt{2}\left(1-2 k_{i}^{2} s_{i}^{2}\right)^{2}} \quad \mathrm{y} \quad d \nu=\frac{d s_{i}}{\sqrt{\left(1-s_{i}^{2}\right)\left(1-k_{i}^{2} s_{i}^{2}\right)}}
$$


Como podemos ver, el valor de la derivada $d t / d \nu$ depende indirectamente del valor de la energía $h_{i}$ mediante $k_{i}=k\left(h_{i}\right)$, se puede considerar que la variable uniformizante o el tiempo dependen de $h_{i}$. En este caso el tiempo es la variable independiente por lo tanto consideraremos $\nu_{i}=\nu\left(h_{i}\right)$ y las expresiones (3.13) deberán leerse como $d t / d \nu_{i}$ y $d \nu_{i}$.

Primero resolveremos la segunda integral directamente, ya que esta es una integral elíptica de primera especie. La solución es $s_{i}=\operatorname{sn}\left(\nu_{i}, k_{i}\right)$ y esta solución se sustituye en la primera integral para obtener la relación entre el viejo y el nuevo tiempo

$$
t=\int \frac{d \nu_{i}}{2 \sqrt{2}\left(1-2 k_{i}^{2} \operatorname{sn}^{2}\left(\nu_{i}, k_{i}\right)\right)^{2}} .
$$

Finalmente, resolviendo para $q_{i}$ en (3.7) y sustituyendo la solución para $s_{i}=\operatorname{sn}\left(\nu_{i}, k_{i}\right)$ se obtiene

$$
q_{i}=k_{i} \frac{s_{i} \sqrt{1-k_{i}^{2} s_{i}^{2}}}{1-2 k_{i}^{2} s_{i}^{2}}=k_{i} \frac{\operatorname{sn}\left(\nu_{i}, k_{i}\right) \operatorname{dn}\left(\nu_{i}, k_{i}\right)}{1-2 k_{i}^{2} \operatorname{sn}^{2}\left(\nu_{i}, k_{i}\right)} .
$$

El momento conjugado se obtendrá al diferenciar la solución anterior y considerar el rescalamiento en el tiempo, es decir

$$
p_{i}=\frac{d q_{i}}{d \nu_{i}} \frac{d \nu_{i}}{d t}=2 \sqrt{2} k_{i} \operatorname{cn}\left(\nu_{i}, k_{i}\right)
$$

Como vemos en el resultado anterior, las soluciones del problema circular de Sitnikov con $2+2$ cuerpos, se escriben en términos de las funciones elípticas de Jacobi $\operatorname{sn}(\nu, k), \operatorname{cn}(\nu, k)$ y $\operatorname{dn}(\nu, k)$ definidas sobre el plano complejo con imagen en la superficie de Riemann $\mathcal{R}_{k}^{1}$ de género 1 . Dicha superficie de Riemann está construida a partir de la diferencial meromorfa

$$
\omega=\frac{d z}{\sqrt{\left(1-z^{2}\right)\left(1-k^{2} z^{2}\right)}},
$$

que se ramifica en los puntos $\{ \pm 1, \pm 1 / k\} \subset \mathbb{C}$.

En el caso general, las funciones elípticas de Jacobi aceptan argumentos y módulos complejos, y se sabe que estas funciones son analíticas con respecto del módulo $k \in \mathbb{C} \backslash\{-1,1\}$ [59]. Nosotros estamos interesados a los casos en los que estas funciones mapean la recta real en ella misma y esto se da si y sólo si $k \in \mathbb{R} \backslash\{-1,1\}$. Los valores de los módulos dependen del valor de las energías relativas mediante las relaciones

$$
k_{3}=\frac{\sqrt{2+\frac{h_{3}}{1+\epsilon}}}{2}, \quad \text { y } \quad k_{4}=\frac{\sqrt{2+\frac{h_{4}}{1-\epsilon}}}{2},
$$

donde $h_{3} \in(-2(1+\epsilon), \infty)$ y $h_{4} \in(-2(1-\epsilon), \infty)$, por lo que se tiene $k_{i} \in(0, \infty)$. 
Si $k_{i} \in(0,1)$ las soluciones se expresan directamente por la ecuación (3.10). Si $k_{i}>1$ debemos considerar transformaciones modulares para mapear el segmento $(0,1) \mapsto(1, \infty)$ mediante $k_{i} \mapsto \frac{1}{k_{i}}$, y sustituir las relaciones

$$
\begin{aligned}
\operatorname{sn}(\nu, k) & =\frac{1}{k} \operatorname{sn}\left(k \nu, \frac{1}{k}\right), \\
\operatorname{cn}(\nu, k) & =\operatorname{dn}\left(k \nu, \frac{1}{k}\right), \\
\operatorname{dn}(\nu, k) & =\operatorname{cn}\left(k \nu, \frac{1}{k}\right),
\end{aligned}
$$

en la expresión (3.10). Finalmente, para el caso particular $k_{i}=1$ la diferencial meromorfa (3.17) estará asociada a una superficie de Riemann $\mathcal{R}_{1}^{0}$ de género 0. Las soluciones se escriben entonces en términos de las funciones hiperbólicas $\sinh (\nu)$ y $\cosh (\nu)$ de la siguiente manera

$$
\begin{aligned}
& \operatorname{sn}(\nu, 1)=\frac{\sinh (\nu)}{\cosh (\nu)}, \\
& \operatorname{cn}(\nu, 1)=\frac{1}{\cosh (\nu)}, \\
& \operatorname{dn}(\nu, 1)=\frac{1}{\cosh (\nu)} .
\end{aligned}
$$

Sustituyendo estos valores en la expresión (3.10) recuperamos las soluciones determinadas por M. Corbera y J. Llibre en [24] para toda $h_{i}>-2$.

Lema 3.6 (Jiménez-Pérez). La integral elíptica incompleta de tercera especie de Jacobi $\Pi(\alpha, \nu, k)$ se puede escribir de la forma

$$
\Pi(\alpha, \nu, k)=(1-\alpha) \int_{0}^{\nu} \frac{d x}{y(x)\left(1-\alpha x^{2}\right)^{2}}+\alpha \int_{0}^{\nu} \frac{\left(1-x^{2}\right) d x}{y(x)\left(1-\alpha x^{2}\right)^{2}},
$$

donde $y(x)=\sqrt{\left(1-x^{2}\right)\left(1-k^{2} x^{2}\right)}$ y $\alpha \in \mathbb{R}$.

Demostración. La integral elíptica incompleta de tercera especie está dada por

$$
\Pi(\alpha, \nu, k)=\int_{0}^{\nu} \frac{d x}{\sqrt{\left(1-x^{2}\right)\left(1-k^{2} x^{2}\right)}\left(1-\alpha x^{2}\right)} .
$$


Si denotamos $y(x)=\sqrt{\left(1-x^{2}\right)\left(1-k^{2} x^{2}\right)}$ entonces podemos escribir sucesivamente

$$
\begin{aligned}
\Pi(\alpha, \nu, k) & =\int_{0}^{\nu} \frac{d x}{y(x)\left(1-\alpha x^{2}\right)} \\
& =\int_{0}^{\nu} \frac{\left(1-\alpha x^{2}\right) d x}{y(x)\left(1-\alpha x^{2}\right)^{2}} \\
& =\int_{0}^{\nu} \frac{\left(1-\alpha+\alpha-\alpha x^{2}\right) d x}{y(x)\left(1-\alpha x^{2}\right)^{2}} \\
& =\int_{0}^{\nu} \frac{\left(1-\alpha+\alpha\left(1-x^{2}\right)\right) d x}{y(x)\left(1-\alpha x^{2}\right)^{2}}, \\
& =(1-\alpha) \int_{0}^{\nu} \frac{d x}{y(x)\left(1-\alpha x^{2}\right)^{2}}+\alpha \int_{0}^{\nu} \frac{\left(1-x^{2}\right) d x}{y(x)\left(1-\alpha x^{2}\right)^{2}}
\end{aligned}
$$

que es el resultado deseado.

La identidad del lema anterior nos servirá para relacionar las expresiones de la acción $J\left(h_{i}\right)$ y del tiempo $t\left(\nu_{i}\right)$, lo que nos permitirá utilizar el mismo cambio de variable que en el Teorema 16. Esta relación se utilizará en la demostración del siguiente resultado.

LEMA 3.7 (Corbera, Llibre, 2002). La relación del tiempo físico con el tiempo rescalado $\nu_{i}, i=3,4$, está dada por

$$
\begin{gathered}
t\left(\nu_{i}, k_{i}\right)=\frac{k_{i}^{2}}{\sqrt{2}\left(1-2 k_{i}^{2}\right)}\left(\frac{1}{4 k_{i}^{2}}\left(2 E\left(\nu_{i}, k_{i}\right)-\nu_{i}+\Pi\left(\nu_{i}, 2 k_{i}^{2}, k_{i}\right)\right)-\right. \\
\left.\frac{\operatorname{sn}\left(\nu_{i}, k_{i}\right) \operatorname{cn}\left(\nu_{i}, k_{i}\right) \operatorname{dn}\left(\nu_{i}, k_{i}\right)}{1-2 k^{2} \operatorname{sn}\left(\nu_{i}, k_{i}\right)^{2}}\right) .
\end{gathered}
$$

Demostración. La relación entre el tiempo físico $t$ y el nuevo tiempo $\nu_{i}$ está determinado por la integral elíptica general siguiente

$$
t\left(\nu_{i}, k_{i}\right)=\frac{1}{2 \sqrt{2}} \int_{0}^{\nu_{i}} \frac{d \eta}{\left(1-2 k_{i}^{2} \operatorname{sn}^{2}\left(\eta, k_{i}\right)\right)^{2}} .
$$

Utilizando el cambio de variable $x=\operatorname{sn}\left(\eta, k_{i}\right)$ escribimos la integral de manera algebraica como

$$
t=\frac{1}{2 \sqrt{2}} \int_{0}^{x_{0}} \frac{d x}{y(x)\left(1-2 k_{i}^{2} x^{2}\right)^{2}},
$$

donde $x_{0}=\operatorname{sn}\left(\nu_{i}, k_{i}\right)$ y $y(x)=\sqrt{\left(1-x^{2}\right)\left(1-k^{2} x^{2}\right)}$. Entonces

$$
2 \sqrt{2} t=\int_{0}^{x_{0}} \frac{d x}{y(x)\left(1-2 k_{i}^{2} x^{2}\right)^{2}} .
$$


La ultima relación en la demostración del Teorema 16 nos indica que

$$
\begin{aligned}
\int_{0}^{x_{0}} \frac{\left(1-x^{2}\right) d x}{y(x)\left(1-2 k_{i}^{2} x^{2}\right)^{2}} d x= & \frac{\operatorname{sn}\left(\nu_{i}, k_{i}\right) \operatorname{cn}\left(\nu_{i}, k_{i}\right) \operatorname{dn}\left(\nu_{i}, k_{i}\right)}{1-2 k_{i}^{2} \operatorname{sn}\left(\nu_{i}, k_{i}\right)^{2}}- \\
& \frac{1}{4 k_{i}^{2}}\left(2 E\left(x_{0}, k_{i}\right)-\Pi\left(2 k_{i}^{2}, x_{0}, k_{i}\right)-F\left(x_{0}, k_{i}\right)\right) .
\end{aligned}
$$

El Lema 4 nos permite relacionar estas expresiones en la forma

$$
\begin{aligned}
\Pi\left(\nu_{i}, 2 k_{i}^{2}, k_{i}\right)= & \left(1-2 k_{i}^{2}\right) 2 \sqrt{2} t+2 k_{i}^{2} \frac{\operatorname{sn}\left(\nu_{i}, k_{i}\right) \operatorname{cn}\left(\nu_{i}, k_{i}\right) \operatorname{dn}\left(\nu_{i}, k_{i}\right)}{1-2 k_{i}^{2} \operatorname{sn}\left(\nu_{i}, k_{i}\right)^{2}}- \\
& \frac{1}{2}\left(2 E\left(x_{0}, k_{i}\right)-\Pi\left(2 k_{i}^{2}, x_{0}, k_{i}\right)-F\left(x_{0}, k_{i}\right)\right) .
\end{aligned}
$$

Despejando la variable $t$ de la última expresión tendremos

$$
\begin{gathered}
t\left(\nu_{i}, k_{i}\right)=\frac{k_{i}^{2}}{\sqrt{2}\left(1-2 k_{i}^{2}\right)}\left(\frac{1}{4 k_{i}^{2}}\left(2 E\left(x_{0}, k_{i}\right)+\Pi\left(2 k_{i}^{2}, x_{0}, k_{i}\right)-F\left(x_{0}, k_{i}\right)\right)\right. \\
\left.-\frac{\operatorname{sn}\left(\nu_{i}, k_{i}\right) \operatorname{cn}\left(\nu_{i}, k_{i}\right) \operatorname{dn}\left(\nu_{i}, k_{i}\right)}{1-2 k_{i}^{2} \operatorname{sn}\left(\nu_{i}, k_{i}\right)^{2}}\right) .
\end{gathered}
$$

Finalmente, tenemos que $x_{0}=\operatorname{sn}\left(\nu_{i}, k_{i}\right)$ y mediante la identidad $F\left(\operatorname{sn}\left(\nu_{i}, k_{i}\right), k_{i}\right)=\nu_{i}$ se obtiene el resultado buscado.

Proposición 3.16 (Corbera, Llibre, 2002). El tiempo de retorno de cada secundario está dado por

$$
T\left(h_{i}\right)=\frac{1}{\sqrt{2}\left(1-2 k_{i}^{2}\right)}\left(2 E\left(k_{i}\right)-K\left(k_{i}\right)+\Pi\left(2 k_{i}^{2}, k_{i}\right)\right)
$$

Demostración. El resultado se obtiene al evaluar la función del tiempo $t\left(\nu_{i}, k_{i}\right)$ en los valores $\nu_{i}=0$ y $\nu_{i}=K\left(k_{i}\right)$ que corresponde a un cuarto de periodo. Entonces $T\left(k_{i}\right)=4\left(t\left(K\left(k_{i}\right), k_{i}\right)-t\left(0, k_{i}\right)\right)$. Como la función se anula en cero $t\left(0, k_{i}\right)=0$ y el coseno elíptico cumple $\operatorname{cn}\left(K\left(k_{i}\right), k_{i}\right)=0$ el último término desaparece y tendremos

$$
t\left(K_{i}, k_{i}\right)-t\left(0, k_{i}\right)=\frac{k_{i}^{2}}{\sqrt{2}\left(1-2 k_{i}^{2}\right)}\left(\frac{1}{4 k_{i}^{2}}\left(2 E\left(K_{i}, k_{i}\right)+\Pi\left(2 k_{i}^{2}, K_{i}, k_{i}\right)-K_{i}\right)\right) .
$$

donde $K_{i}=K\left(k_{i}\right)$. Directamente obtenemos el resultado al reacomodar los términos, verificar que $E\left(K\left(k_{i}\right), k_{i}\right)=E\left(k_{i}\right), \Pi\left(2 k_{i}^{2}, K\left(k_{i}\right), k_{i}\right)=\Pi\left(2 k_{i}^{2}, k_{i}\right)$ y multiplicar por 4 el resultado obtenido. Es decir $T\left(h_{i}\right)=4 t\left(K\left(k_{i}\right)\right)$.

Nota 3.5. Este resultado también se puede obtener mediante las coordenadas acción-ángulo ya que el periodo (de cada secundario independiente) es proporcional a la derivada de la coordenada de acción con respecto de la energía

$$
T\left(h_{i}\right)=2 \pi \frac{\partial J_{i}}{\partial h_{i}}\left(h_{i}\right)
$$


Aunque este resultado no es difícil de obtener, se requiere un poco más de herramientas de las derivadas de las funciones elípticas consideradas como funciones analíticas del módulo $k_{i}$. En particular, los capítulos 3 y 6 del libro de Lawden [59] contienen toda la herramienta teórica para realizar dichos cálculos.

\subsection{HiPER-SUPERFICIES DE ENERGÍA CONSTANTE.}

En esta sección describimos la topología de los conjunto de nivel para la función Hamiltoniana (3.1) para $\epsilon \in[0,1)$, con la ayuda de la aplicación momento y de su imagen. Primero demostraremos un resultado relacionado con la estructura de las superficies de nivel de un sistema Hamiltoniano con función $H$ separable y posteriormente utilizaremos este resultado en nuestro caso particular.

3.3.1. Hamiltonianos completamente separables. Supongamos que se tiene un sistema Hamiltoniano $\left(M, \omega, X_{H}\right)$ completamente integrable sobre la variedad simpléctica $(M, \omega)$ de, dimensión $2 n$. Esto nos indica que existen $n$ integrales primeras $\left\{F_{i}\right\}_{i=1}^{n}$ donde $F_{1}=H$ y las cuales son independientes y están en involución, es decir, que el determinante del Wronskiano no se anule y que $\left\{F_{i}, F_{j}\right\}=0$ para $i, j \in\{1,2, \ldots, n\}$ con $i \neq j$.

Consideremos ahora una aplicación momento $\mu: M \rightarrow \mathfrak{g}^{*} \cong \mathbb{R}^{n}$ definida por las integrales primeras como componentes de la siguiente manera

$$
\mu=\left(F_{1}=H, F_{2}, \ldots, F_{n}\right)
$$

Si consideramos un punto en la imagen de la aplicación y buscamos su imagen inversa lo que obtendremos son subvariedades Lagrangianas, una por cada punto en la imagen de $\mu$. Dicho de otra forma, se tiene que para cada $x \in \operatorname{Img}(\mu)$ la fibra $\mu^{-1}(x)$ es una subvariedad Lagrangiana, que puede ser un toro, un cilindro o un plano. En este sentido, tendremos una fibración Lagrangiana

Primero probaremos un resultado relacionado con la imagen de las superficies de energía constante de sistemas Hamiltonianos completamente integrables (separables) bajo su aplicación momento.

Proposición 3.17 (Jiménez-Pérez). Sea $(M, \omega)$ una variedad simpléctica exacta de dimensión $2 n$, y sea $\mathcal{H}=\left(M, \omega, X_{H}\right)$ un sistema Hamiltoniano sobre $(M, \omega)$. Supongamos que existe un simplectomorfismo $\rho: M \rightarrow M$ tal que la nueva función Hamiltoniana $F=H \circ \rho$ es separable. Entonces, existe una fibración Lagrangiana $\pi: M \rightarrow \mathbb{R}^{n}$ tal que las superficies de energía constante $\Sigma_{h}$ se mapean a hiperplanos de $\mathbb{R}^{n} \cong \mathfrak{g}^{*}$ que son perpendiculares al vector $\mathbb{1}:=(1,1, \ldots, 1) \in \mathbb{R}^{n}$.

Demostración. Como existe un simplectomorfismo $\rho: M \rightarrow M$ tal que $F=H \circ \rho$ es separable entonces existen coordenadas globales $(Q, P)$ 
donde $F(Q, P)=H(\rho(Q, P))$ se puede escribir de la forma

$$
F(Q, P)=F_{1}\left(Q_{1}, P_{1}\right)+\cdots+F_{n}\left(Q_{n}, P_{n}\right)
$$

donde $F_{i}\left(Q_{i}, P_{i}\right)=$ constante son $n$ integrales primeras para $X_{F}$. Además $\left\{F, F_{i}\right\}=0$ para $i=1, \ldots, n$. El sistema Hamiltoniano es integrable por cuadraturas y podemos considerar el flujo combinado $\varphi^{\mathbf{t}}$ de todos los campos vectoriales Hamiltonianos $X_{F_{i}}$ como una acción del grupo de Lie $G=\mathbb{R}^{k} \times$ $\mathbb{T}^{n-k}$ sobre $(M, \omega)$ para alguna $1 \leq k \leq n$.

Como $G$ es conmutativo, la acción Hamiltoniana $G \times M \rightarrow M$ induce una aplicación momento

$$
\mu=\left(F_{1}, F_{2}, \ldots, F_{n}\right): M \rightarrow \mathfrak{g}^{*}
$$

donde $\mathfrak{g}^{*} \cong \mathbb{R}^{n}$ es el espacio dual del álgebra de Lie $\mathfrak{g}=\operatorname{Lie}(G)$ asociada a $G=\varphi^{\mathrm{t}}$. (En realidad sólo se requiere que la acción adjunta de $G$ sobre $\mathfrak{g}^{*}$ sea equivariante.) Su imagen $\operatorname{Img}(\mu) \subset \mathbb{R}^{n}$ es un poliedro convexo, un ortante o una combinación de ambos, cuyos vértices son los valores extremos de la aplicación $\mu$ 36. La imagen $\mu(\Sigma)$ de cada superficie regular de energía constante $\Sigma=F^{-1}(h)$ bajo la aplicación momento corresponde a un subconjunto convexo $\mathcal{K}$ de un subespacio afín de codimensión 1 de $\mathfrak{g}^{*}$ dado por

$$
x_{1}+x_{2}+\cdots+x_{n}-h=0
$$

donde $\mathbf{x}=\left(x_{1}, \ldots, x_{n}\right) \in \mathbb{R}^{n}$. De aquí, podemos escribir $\langle\mathbf{x}, \mathbb{1}\rangle-h=0$, y en particular tendremos

$$
\mathcal{K}:=\left\{\mathbf{x} \in \mathbb{R}^{n} \mid \mathbf{x} \in(\operatorname{Img}(\mu) \cap\langle\mathbf{x}, \mathbb{1}\rangle=h)\right\} \subset\left\{\mathbf{x} \in \mathbb{R}^{n} \mid\langle\mathbf{x}, \mathbb{1}\rangle=h\right\}
$$

Entonces $\pi:=\mu \circ \rho: M \rightarrow \mathbb{R}^{n}$ es la aplicación suave que estamos buscando.

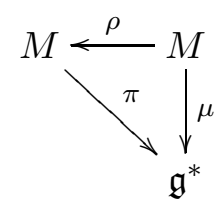

Solo falta probar que $\pi^{-1}(\mathbf{x})$ es una subvaridad Lagrangiana para toda $\mathbf{x} \in \operatorname{Img}(\mu) \subset \mathfrak{g}^{*}$. Sabemos que las fibras $\mathfrak{L}=\mu^{-1}(\mathbf{x})$ donde $\mathbf{x} \in \mathfrak{g}^{*}$ son subvariedades Lagrangianas de $M$ para cada $\mathbf{x} \in \operatorname{Img}(\mu)$, esto implica que $\left.\omega\right|_{\mathfrak{L}} \equiv 0$. Finalmente se tiene que $\rho^{-1} \in S p(M, \omega)$ si y sólo si $\left(\rho^{-1}\right)^{*} \omega=\omega$, entonces

$$
\omega(x, y)=\omega\left(\rho^{-1}(x), \rho^{-1}(y)\right)=0, \quad \forall x, y \in \mathfrak{L}
$$

por lo tanto $\hat{\mathfrak{L}}=\rho^{-1}(\mathfrak{L})$ es también subvariedad Lagrangiana. Concluimos que $\pi: M \rightarrow \mathbb{R}^{n}$ es una fibración Lagrangiana.

Nota 3.6. Es importante ver que los puntos interiores del conjunto $\mathcal{K}$ corresponden a subvariedades Lagrangianas de $M$. Por otro lado, los puntos que se encuentran sobre la frontera $\partial \mathcal{K}$ corresponden a subvariedades isotrópicas que tienen la forma $\mathbb{R}^{r} \times \mathbb{T}^{s}$ con $0 \leq r, s<n$ y $r+s<n$. 
3.3.2. Foliación DEL ESPACIO FASE POR SUPERFICIES DE ENERGÍA CONSTANTE. Para aplicar el teorema anterior a nuestro problema, consideramos que nuestro sistema es completamente integrable según Liouville, y además tiene una función Hamiltoniana separable $H=H_{3}+H_{4}$, y modificaremos la selección de integrales primeras para tener a la aplicación momento en una forma simétrica.

DeFinición 3.1. La aplicación momento $\mu: M \rightarrow \mathfrak{g}^{*}$ para el problema circular-colineal restringido de $2+2$ cuerpos está dada por

$$
\begin{aligned}
\mu: M & \rightarrow \mathfrak{g}^{*} \cong \mathbb{R}^{2} \\
\left.q_{3}, q_{4}, p_{3}, p_{4}\right) & \mapsto\left(\frac{1}{2(1+\epsilon)} p_{3}^{2}-\frac{1+\epsilon}{\sqrt{q_{3}^{2}+1 / 4}}, \frac{1}{2(1-\epsilon)} p_{4}^{2}-\frac{1-\epsilon}{\sqrt{q_{4}^{2}+1 / 4}}\right),
\end{aligned}
$$

donde $\epsilon \in[0,1)$

Utilizando la proposición anterior tenemos de manera inmediata el siguiente resultado

Proposición 3.18 (Jiménez-Pérez). Las superficies de energía constante del problema circular restringido de Sitnikov con $2+2$ cuerpos, bajo la aplicación momento (3.22) corresponden a segmentos de línea con pendiente $m=-1$ en $\operatorname{Img}(\mu) \subset \mathbb{R}^{2}$

Demostración. El problema circular restringido de Sitnikov de $2+2$ cuerpos es un sistema Hamiltoniano separable sobre la variedad $M \cong \mathbb{R}^{4}$. Entonces, la imagen de la aplicación (3.22) es un subespacio afín de $\mathbb{R}^{2}$ que es perpendicular al vector $\mathbb{1}_{\mathbb{R}^{2}}=(1,1)$. Por lo tanto son líneas rectas con pendiente $m=-1$.

Ahora describiremos las superficies de energía constante y sus foliaciones, para lo cual, consideraremos la función Hamiltoniana separable de la siguiente forma

$$
H(\mathbf{q}, \mathbf{p})=h=h_{3}+h_{4}
$$

donde $h_{i}$ corresponde a un valor fijo de la energía para el problema circular clásico de Sitnikov, para cada $i=3,4$. Entonces consideramos el producto cartesiano que corresponderá a la aplicación momento $\mathbf{x}=\left(h_{3}, h_{4}\right) \in \operatorname{Img}(\mu)$ y finalmente construimos la foliación siguiendo las líneas rectas asociadas a cada nivel de energía en $\operatorname{Img}(\mu)$.

Primero debemos establecer la relación entre $h_{3}$ y $h_{4}$ puesto que son dos problemas equivalentes, pero con diferente energía. Consideremos $h_{0}$ la energía del caso $\epsilon=0$. Entonces $H_{0}$ es exactamente un problema circular de Sitnikov dado por

$$
H_{0}=\frac{1}{2} p^{2}-\frac{1}{\sqrt{q^{2}+1 / 4}}
$$


Haciendo un reescalamiento lineal en el tiempo $\hat{t}=(1 \pm \epsilon) t$ y una transformación simpléctica con multiplicador $(1 \pm \epsilon)$ tenemos que

$$
H_{3}=(1+\epsilon) H_{0} \quad \text { y } \quad H_{4}=(1-\epsilon) H_{0} .
$$

y las relaciones en el tiempo son $t=\frac{1}{(1+\epsilon)} t_{3}=\frac{1}{(1-\epsilon)} t_{4}$ de donde tenemos que $t_{4}=c t_{3} \operatorname{con} c=\frac{1-\epsilon}{1+\epsilon}$.

Utilizando las soluciones para el problema circular de Sitnikov [8, sabemos que $h_{0}$ tienen su imagen en $[-2, \infty)$ y las órbitas tienen el siguiente comportamiento: si $-2<h_{0}<0$ el problema circular de Sitnikov tiene órbitas periódicas, para $h_{0}=0$ tiene una órbita parabólica mientras que para $h_{0}>0$ se tienen órbitas hiperbólicas. Debido a la restricción para cada valor de la energía relativa $h_{i}, i \in\{3,4\}$, la energía del sistema

$$
h=h_{3}+h_{4}=(1+\epsilon) h_{0}+(1-\epsilon) h_{0}=2 h_{0}
$$

tiene su imagen en $(-4, \infty) \subset \mathbb{R}$. En el caso $\epsilon>0$ tenemos que la imagen de la aplicación momento es el subconjunto

$$
\mathcal{M}=\left\{\left(h_{3}, h_{4}\right) \in \mathbb{R}^{2} \mid-2(1+\epsilon)<h_{3},-2(1-\epsilon)<h_{4}\right\}
$$

En la Figura 3.1 puede verse este conjunto y las líneas cuya imagen inversa corresponden a los niveles de energía fija del sistema.

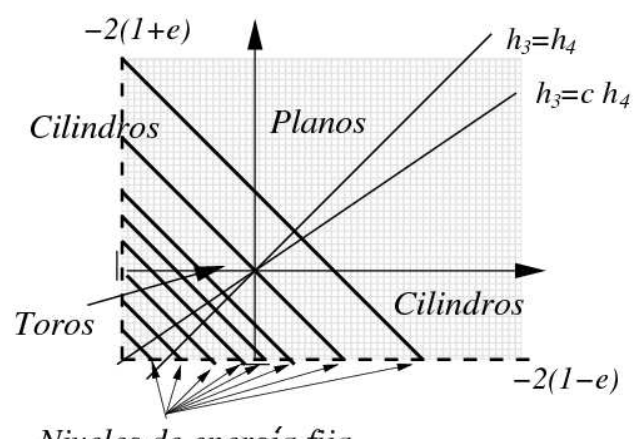

Niveles de energía fija

Figura 3.1. Líneas de los niveles de energía, $\epsilon>0$.

Considerando que cada punto de las líneas corresponde a un toro, un cilindro o un plano dependiendo del cuadrante donde se encuentre podemos ver como es la foliación de los niveles de energía. Para el caso $\epsilon>0$ tenemos que las superficies de nivel equivalen topológicamente a los siguientes objetos:

- Si $h=-4$ este nivel de energía no existe en el problema real ya que se encuentra en el conjunto de singularidades $\Delta$ (los secundarios están en el origen en el mismo tiempo: imposible) 
- Si $-4<h<-2(1+\epsilon)$ los niveles de energía corresponden topológicamente a esferas $\mathbb{S}^{3}$ foliadas por toros $\mathbb{T}^{2}$ y contienen dos curvas cerradas singulares en los extremos.

- Si $h=-2(1+\epsilon)$ la superficie de energía es una esfera $\mathbb{S}^{3}$ agujereada en 2 puntos.

- Si $-2(1+\epsilon)<h<-2(1-\epsilon)$ las superficies de energía son esfera $\mathbb{S}^{3}$ con 2 discos $\mathbb{D}^{2}$ como frontera.

- Si $h=-2(1-\epsilon)$ la superficie de energía es una esfera $\mathbb{S}^{3}$ con 2 discos $\mathbb{D}^{2}$ como frontera y agujereada en 2 puntos adicionales.

- Si $-2(1-\epsilon)<h<0$ las superficies de energía son esferas $\mathbb{S}^{3}$ con 4 discos $\mathbb{D}^{2}$ como frontera.

- Si $h=0$ la foliación contiene cilindros disjuntos que contienen a cuatro planos en el punto medio (cuando $h_{3}=h_{4}=0$ ).

- Si $h>0$ la foliación contiene cilindros y planos únicamente.

Algunas de estas foliaciones se muestran en la figura 3.2

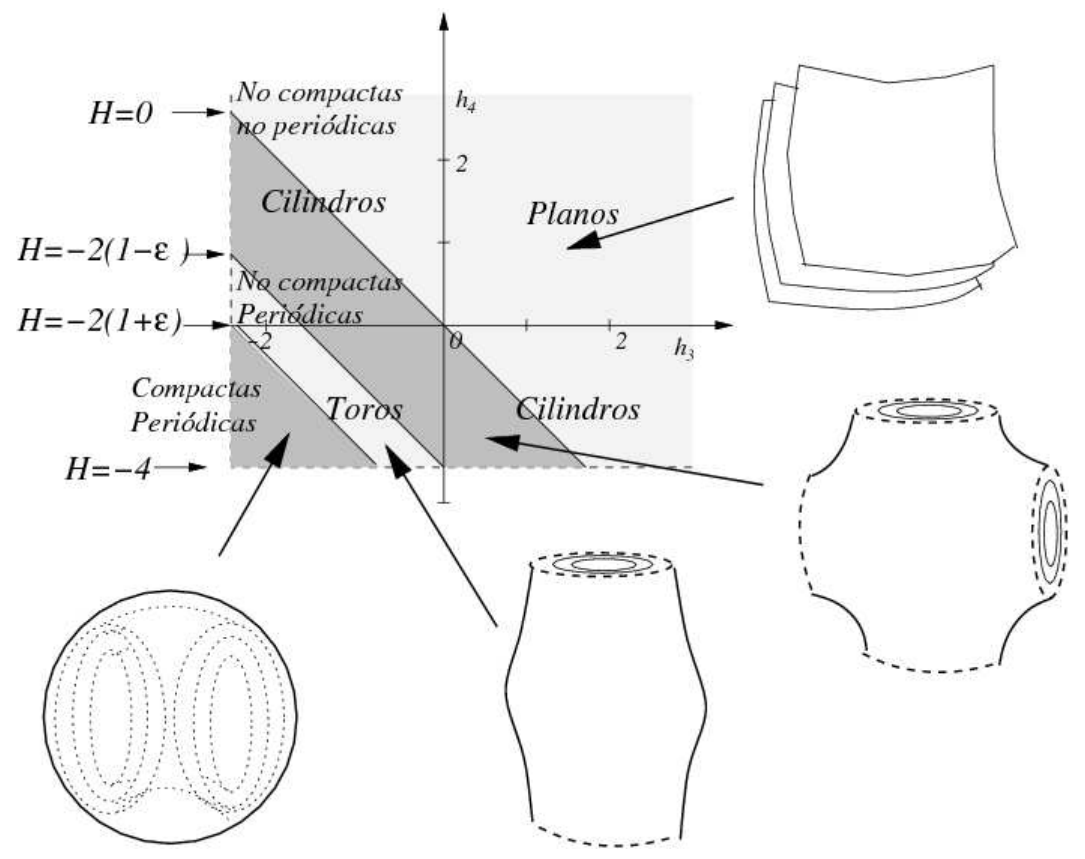

Figura 3.2. Foliación de los niveles de energía constante, $\epsilon>0$.

Es importante notar que cuando $\epsilon \rightarrow 0$ entonces la franja que se encuentra entre $-2(1+\epsilon)<h<-2(1-\epsilon)$ desaparece y los casos extremos se unen en uno sólo. De esta forma, la foliación para el caso $\epsilon=0$ es un poco más simple:

- Si $h=-4$ este nivel de energía no existe en el problema real ya que se encuentra en el conjunto de singularidades $\Delta$ (los secundarios están en el origen en el mismo tiempo: imposible) 
- $\mathrm{Si}-4<h<-2$ los niveles de energía corresponden topológicamente a esferas $\mathbb{S}^{3}$ foliadas por toros $\mathbb{T}^{2}$ y contienen dos curvas cerradas singulares en los extremos.

- Si $h=-2$ la superficie de energía es una esfera $\mathbb{S}^{3}$ agujereada en 4 puntos.

- Si $-2<h<0$ las superficies de energía son esferas $\mathbb{S}^{3}$ con 4 discos $\mathbb{D}^{2}$ como frontera.

- Si $h=0$ la foliación contiene cilindros disjuntos que contienen a cuatro planos en el punto medio (cuando $h_{3}=h_{4}=0$ ).

- Si $h>0$ la foliación contiene cilindros y planos únicamente.

Algunas de estas foliaciones se muestran en la figura 3.3 .

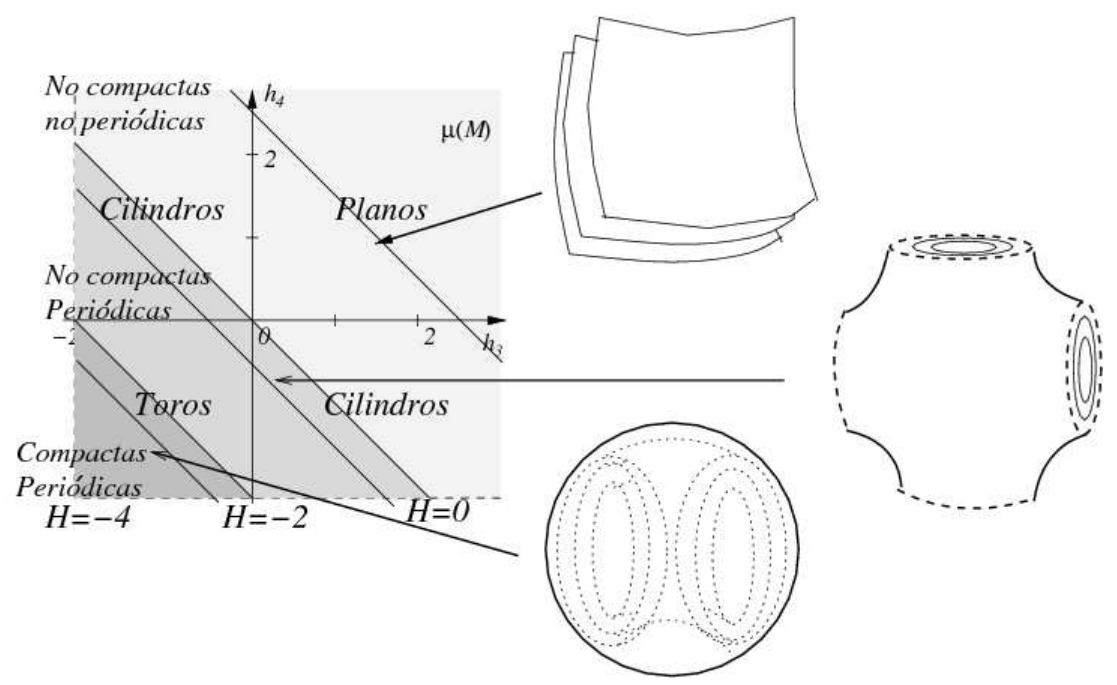

Figura 3.3. Foliación de los niveles de energía constante, $\epsilon=0$.

Por supuesto, los niveles de energía más interesantes son $h=-2(1+\epsilon)$, $h=-2(1-\epsilon)$ y $h=0$ ya que estos son valores de bifurcación para la topología de las superficies de nivel. Otros valores interesantes para la energía son $-4<h<-2(1+\epsilon)$ ya que las superficies de energía son equivalentes a esferas $\mathbb{S}^{3}$ foliadas por toros de dimensión 2 y tienen todas sus soluciones acotadas. Muchas de ellas ofrecen la posibilidad de encontrar soluciones periódicas interesantes que se preservarán bajo pequeñas perturbaciones del sistema; ya sea a través de perturbaciones a la excentricidad $e$ de las órbitas keplerianas o de perturbaciones en el parámetro $\epsilon$. 


\subsection{El CONJUNTO DE COLISión.}

El conjunto de las colisiones para el problema de Sitnikov con $2+2$ cuerpos es el hiperplano $\Delta$. Este conjunto es una variedad, aunque no corresponde con el concepto de variedad de colisión definida por McGehee en 71].

Cuando se considera el problema con atracción entre los secundarios, se debe utilizar el sistema regularizado dado en las expresiones (2.33) y (2.35). En este caso, la intersección de una superficie de energía constante con el conjunto $\Delta$ corresponde a subvariedades no compactas equivalentes a esferas bidimensionales con 2, 4 o 6 finales cilíndricos, dependiendo del valor de la energía y de las masas infinitesimales.

Si las masas de los cuerpos infinitesimales son diferentes $m_{3} \neq m_{4}$, entonces ellos deben experimentar un intercambio de momento al pasar por la colisión. Sin embargo, la regularización reduce el conjunto de colisiones en 1 dimensión y es díficil determinar la continuación de las soluciones de manera única. Para comprender mejor la forma de hacer esa continuación haremos un estudio detallado del caso sin atracción entre los cuerpos infinitesimales. Esto nos lleva al caso integrable donde el conjunto de colisiones tiene codimensión 1 y la intersección con lo niveles de energía fija $h$ son variedades compactas cuando $h<0$.

Proposición 3.19 (Jiménez-Pérez). El conjunto de colisiones para una superficie de energía fija $H(\mathbf{q}, \mathbf{p})=h$ es topológicamente equivalente a:

- una esfera $\mathbb{S}^{2}$ de dimensión 2 si $h \in(-4,0)$,

- una esfera $\mathbb{S}^{2} \backslash\left\{x_{+}, x_{-}\right\}$de dimensión 2 sin dos puntos para $h=0$,

- un cilindro $\mathcal{C} \cong \mathbb{S}^{1} \times \mathbb{R}$ de dimensión 2 si $h>0$

Demostración. Fijemos un nivel de energía $H(\mathbf{q}, \mathbf{p})=h$ y consideremos la intersección de la superficie de nivel con el conjunto de colisiones

$$
\Sigma_{h} \cap \Delta .
$$

La función Hamiltoniana para $q \in \Delta$ se convierte en

$$
h=\frac{1}{2(1+\epsilon)} p_{3}^{2}+\frac{1}{2(1-\epsilon)} p_{4}^{2}-\frac{2}{\sqrt{q^{2}+1 / 4}},
$$

de donde obtenemos la relación

$$
0 \leq h+\frac{2}{\sqrt{q^{2}+1 / 4}}=\frac{1}{2(1+\epsilon)} p_{3}^{2}+\frac{1}{2(1-\epsilon)} p_{4}^{2} .
$$

Si $p_{i}=0$ para $i=3$ o $i=4$ se tiene la proyección de curvas equivalentes a las curvas de nivel del problema de Sitnikov clásico. Por otro lado, para cada $q=q_{0}$ fija se tienen como secciones elipses con semiejes $a$ y $b$ dados 
por

$$
\begin{aligned}
& a=\sqrt{2(1+\epsilon)\left(h+2 / \sqrt{q_{0}^{2}+1 / 4}\right)}, \\
& b=\sqrt{2(1-\epsilon)\left(h+2 / \sqrt{q_{0}^{2}+1 / 4}\right)} .
\end{aligned}
$$

Esto nos indica que para ciertos valores de $h$ se tienen superficies topológicamente equivalentes a esferas y para otros las superficies son equivalentes a cilindros. Determinemos los valores de $h$ para los cuales se tienen se tiene cada una de las equivalencias anteriores.

La relación (3.25) determina el intervalo válido para $q$. Los extremos de este intervalo se encuentran al considerar la igualdad $0=h+\frac{2}{\sqrt{q^{2}+1 / 4}} \mathrm{y}$ obtener $q$ como función de $h$.

Explícitamente los extremos son

$$
q(h)= \pm \sqrt{\frac{4}{h^{2}}-\frac{1}{4}}
$$

y los valores de $q$ están en el intervalo

$$
-\sqrt{\frac{4}{h^{2}}-\frac{1}{4}} \leq q \leq \sqrt{\frac{4}{h^{2}}-\frac{1}{4}} .
$$

Inmediatamente vemos los siguientes casos:

- Si $h<-4$ el intervalo es vacio y no existe conjunto de colisiones.

- Si $h=-4$ se tiene un punto y el conjunto de colisión es un punto también.

- Si $-4<h<0$ el intervalo es acotado y por lo tanto los conjuntos de colisiones son superficies compactas, cerradas y simplemente conexas. Es decir

$$
\left(\Sigma_{h} \cap \Delta\right) \cong \mathbb{S}^{2} .
$$

- Si $h=0$ el intervalo corresponde a todo el eje de las qs y las proyecciónes en $p_{i}=0, i=3,4$, corresponden a las órbitas parabólicas del problema de Sitnikov clásico. Entonces

$$
\left(\Sigma_{h} \cap \Delta\right) \cong\left(\mathbb{S}^{2} \backslash\left\{x_{-\infty}, x_{\infty}\right\}\right) .
$$

donde $x_{-\infty}=(-\infty, 0,0)$ y $x_{\infty}=(\infty, 0,0)$.

- Si $h>0$ el intervalo corresponde a todo el eje de las qs y las proyecciónes en $p_{i}=0, i=3,4$, corresponden a las órbitas hiperbólicas del problema de Sitnikov clásico. Entonces

$$
\left(\Sigma_{h} \cap \Delta\right) \cong \mathbb{S}^{1} \times \mathbb{R}
$$


Para estudiar el comportamiento del sistema al pasar a través de una colisión, estudiaremos con más detalle cómo se descompone el conjunto de colisiones en curvas asociadas a los toros invariantes Lagrangianos. Esta descomposición se puede ver como una foliación por curvas obtenidas por la intersección de cada fibra de la aplicación momento $\mu^{-1}(x)$ con el conjunto de colisiones. Estaremos interesados en los valores de la función Hamiltoniana $H(\mathbf{q}, \mathbf{p})=h$ tal que $h<0$ dado que en esa región existen órbitas periódicas y el conjunto de colisiones es un conjunto acotado difeomorfo a $\mathbb{S}^{2}$, como se estableció en la sección 3.3.2 y en la Proposición 3.19

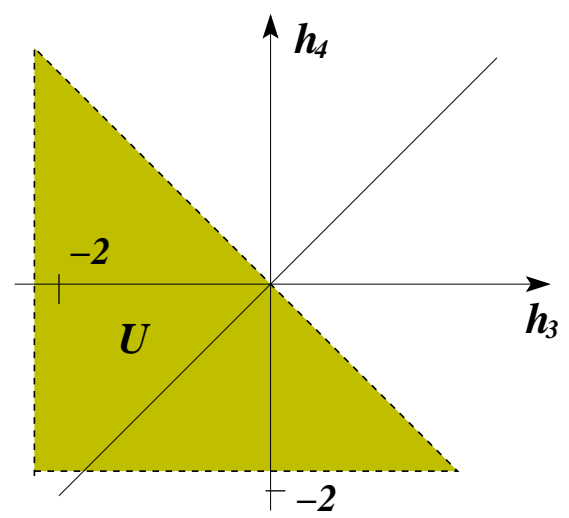

Figura 3.4. El subconjunto de $\operatorname{Im}(\mu)$ que cumple $h<0$.

Sea $U \subset \operatorname{Im}(\mu)$ un sunconjunto abierto en la imagen de la aplicación momento (3.22), determinado por las condiciones

$$
h_{3}>-2(1+\epsilon), \quad h_{4}>-2(1-\epsilon) \quad \text { y } \quad h_{3}<-h_{4},
$$

como se muestra en la Figura 3.4. Para cada $x \in U$, donde $x=\left(h_{3}, h_{4}\right)$ la fibra $\mathscr{T}_{x}=\mu^{-1}(x)$ corresponde a un toro o a un cilindro invariante en $\mathbb{R}^{4}$ donde se encuentran las soluciones del problema integrable con energías relativas $h_{3}$ y $h_{4}$. Designemos por $\mathcal{C}_{x}=\mathscr{T}_{x} \cap \Delta$ al conjunto formado por la intersección de la fibra $\mathscr{T}_{x}$ con el conjunto de colisiones. Entonces se tienen los siguientes resultados

LEMA 3.8. La proyección del conjunto $\mathcal{C}_{x}$ sobre el subespacio de momentos $\left(p_{3}, p_{4}\right)$ corresponde a segmentos de

1. dos rectas transversales en el origen con pendientes $p_{4}= \pm \sqrt{\frac{1-\epsilon}{1+\epsilon}} p_{3}$ cuando $h_{3}=h_{4}$,

2. las dos hojas de una hipérbola asintótica a las rectas del punto anterior cuando $h_{3} \neq h_{4}$.

Demostración. A partir de la función Hamiltoniana separable (3.1) vemos que la intersección de la fibra $\mathscr{T}_{x}$ con el conjunto de colisiones $\Delta$ 
está determinado por las ecuaciones

$$
\begin{aligned}
& h_{3}=\frac{1}{2(1+\epsilon)} p_{3}^{2}-\frac{1}{\sqrt{q^{2}+1 / 4}}, \\
& h_{4}=\frac{1}{2(1-\epsilon)} p_{4}^{2}-\frac{1}{\sqrt{q^{2}+1 / 4}} .
\end{aligned}
$$

donde $q \in \Delta$. Restando las 2 ecuaciones para eliminar la variable $q$ obtenemos

$$
h_{3}-h_{4}=\frac{1}{2(1+\epsilon)} p_{3}^{2}-\frac{1}{2(1-\epsilon)} p_{4}^{2} .
$$

La ecuación (3.29) corresponde a la proyección del conjunto $\mathcal{C}_{x}$ sobre el plano $\left(p_{3}, p_{4}\right)$. Ahora obtenemos los siguientes casos

1. si $h_{3}=h_{4}$ entonces la expresión (3.29) se descompone en las ecuaciones

$$
\begin{aligned}
& \frac{1}{\sqrt{2(1+\epsilon)}} p_{3}-\frac{1}{\sqrt{2(1-\epsilon)}} p_{4}=0 \\
& \frac{1}{\sqrt{2(1+\epsilon)}} p_{3}+\frac{1}{\sqrt{2(1-\epsilon)}} p_{4}=0
\end{aligned}
$$

que corresponde a las dos rectas transversales $p_{4}= \pm \sqrt{\frac{1-\epsilon}{1+\epsilon}} p_{3}$.

2. si $h_{3} \neq h_{4}$ la ecuación (3.29) es una hipérbola asintótica a las rectas del punto anterior.

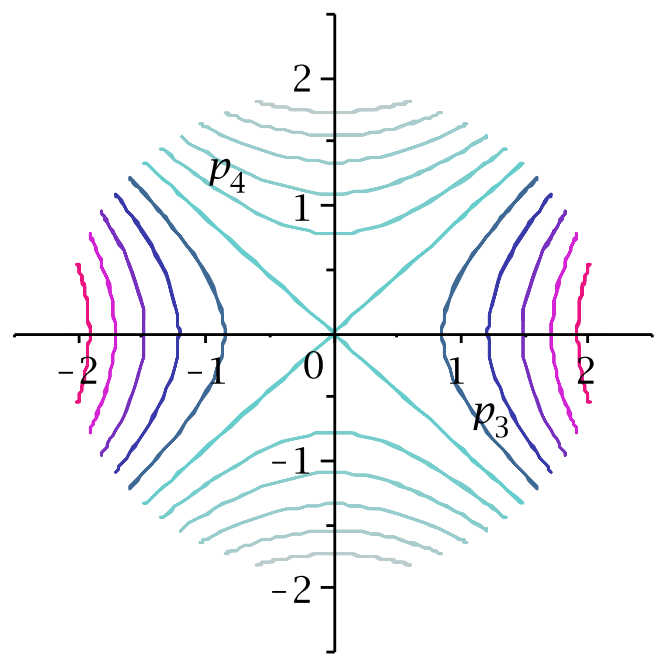

Figura 3.5. Proyección del conjunto de colisiones de los toros invariantes sobre $\left(p_{3}, p_{4}\right)$ con $\epsilon=0.1$. 
Las curvas anteriores están sujetas a la condición

$$
4+\left(h_{3}+h_{4}\right) \leq \frac{1}{2(1+\epsilon)} p_{3}^{2}+\frac{1}{2(1-\epsilon)} p_{4}^{2},
$$

que es el interior de la elipse con semiejes $a$ y $b$, dados en (3.26) y (3.27) respectivamente, y donde $h=h_{3}+h_{4}$. En la Figura 3.5 se muestran las hipérbolas obtenidas mediante la proyección del conjunto $\mathcal{C}_{x}$ para diversos valores de $x=\left(h_{3}, h_{4}\right)$ sobre el mismo nivel de energía $h=h_{3}+h_{4}$.

DEFInICIÓn 3.2. La proyección canónica del espacio fase del problema circular de Sitnikov en el subespacio simpléctico $\mathcal{S}_{i}=\left(q_{i}, p_{i}\right)$ está dada por

$$
\begin{aligned}
\pi_{i}: M & \rightarrow \mathcal{S}_{i}, \\
\left(q_{3}, p_{3}, q_{4}, p_{4}\right) & \mapsto\left(q_{i}, p_{i}\right), \quad i=3,4 .
\end{aligned}
$$

Nota 3.7. El lector debe considerar en los siguientes resultados que la proyección $\pi_{i}\left(\Sigma_{h} \cap \Delta\right) \subset \mathcal{S}_{i}$ tiene coordenadas $\left(q_{3}, p_{3}, 0,0\right)$ o $\left(0,0, q_{4}, p_{4}\right)$. Por lo tanto el subindice $i$ aparece en la variable $q_{i}$ en casi todas las expresiones de lo que resta de la sección. Cuando el subindice no aparezca en q, entonces se trata de un subconjunto de $\Sigma_{h} \cap \Delta$.

El siguiente resultado determina cómo es la proyección del conjunto de colisiones $\mathcal{C}_{x}$ en cada subespacio simpléctico.

Lema 3.9. La proyección $\pi_{i}\left(\mathcal{C}_{x}\right)$ del conjunto $\mathcal{C}_{x}$ en el subespacio simpléctico $\mathcal{S}_{i}, i=3,4$, corresponde a uno de los dos casos siguientes:

1. Si $h_{i} \leq h_{j}, j=3,4$, y $i \neq j$, entonces $\pi_{i}\left(\mathcal{C}_{x}\right)$ corresponde a una curva de nivel completa del espacio fase del problema circular de Sitnikov clásico.

2. Si $h_{i}>h_{j}$, entonces $\pi_{i}\left(\mathcal{C}_{x}\right)$ corresponderá a dos segmentos de una curva de nivel del problema de Sitnikov clásico determinados por la condición

$$
-\sqrt{\frac{1}{h_{j}^{2}}-\frac{1}{4}} \leq q_{i} \leq \sqrt{\frac{1}{h_{j}^{2}}-\frac{1}{4}} .
$$

Demostración. Consideremos el conjunto de colisiones para un nivel de energía $H=h<0$, dado por la ecuación (3.24) y el conjunto $\mathcal{C}_{x}$ asociado a la fibra $\mathscr{T}_{x}$ para $x=\left(h_{3}, h_{4}\right)$ tal que $h=h_{3}+h_{4}$. A partir de las expresiones (3.28) vemos directamente que $\pi_{i}\left(\mathcal{C}_{x}\right)$ corresponde a una curva de nivel del problema circular de Sitnikov clásico. Designemos por $\Gamma_{i}=\Gamma_{i}\left(h_{i}\right)$ a la curva de nivel del problema circular de Sitnikov clásico con energía $h_{i}$, para $i=$ 3,4 . Dicha curva de nivel puede o no estar completamente contenida en la proyección $\pi_{i}\left(\Sigma_{h} \cap \Delta\right)$ del conjunto de colisión. Una curva sobre $\Sigma_{h} \cap \Delta$ alcanza la frontera en $\pi_{i}\left(\Sigma_{h} \cap \Delta\right) \subset \mathcal{S}_{i}$ cuando $p_{j}=0, j=3,4$ e $i \neq j$. Por lo tanto, la frontera de $\pi_{i}\left(\Sigma_{h} \cap \Delta\right)$ está dada por la curva

$$
h=\frac{1}{2\left(1+\varepsilon_{i}\right)} p_{i}^{2}-\frac{2}{\sqrt{q_{i}^{2}+1 / 4}},
$$


donde $\varepsilon_{i}=(-1)^{i+1} \epsilon$. Dicha curva servirá como frontera de la proyección del conjunto $\mathcal{C}_{x}$ para cualquier fibra $\mathscr{T}_{x}$ dada.

Considerando que

$$
0 \leq h+\frac{2}{\sqrt{q_{i}^{2}+1 / 4}}=\frac{1}{2\left(1+\varepsilon_{i}\right)} p_{i}^{2},
$$

entonces la variable $q_{i}$ está restringida a

$$
-\sqrt{\frac{1}{\left(\frac{h_{i}+h_{j}}{2}\right)^{2}}-\frac{1}{4}} \leq q_{i} \leq \sqrt{\frac{1}{\left(\frac{h_{i}+h_{j}}{2}\right)^{2}}-\frac{1}{4}} .
$$

Veamos ahora cada caso independiente.

1. Supongamos que $h_{i} \leq h_{j}$, directamente tenemos $h_{i} \leq \frac{h_{i}+h_{j}}{2}<0$ que implica $h_{i}^{2} \geq\left(\frac{h_{i}+h_{j}}{2}\right)^{2}$. Entonces los valores que puede tomar $q_{i}$ estan acotados por

$$
\left|q_{i}\right| \leq \sqrt{\frac{1}{h_{i}^{2}}-\frac{1}{4}} \leq \sqrt{\frac{1}{\left(\frac{h_{i}+h_{j}}{2}\right)^{2}}-\frac{1}{4}} .
$$

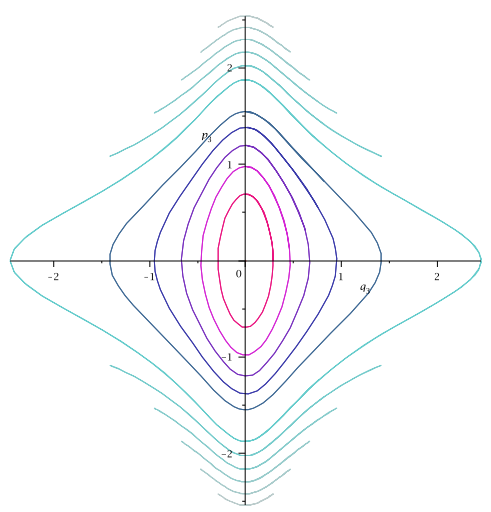

Figura 3.6. Proyección del conjunto de colisiones $\mathcal{C}_{x}$ sobre $\left(q_{3}, p_{3}\right)$. Su frontera es la curva (3.31).

Además, de las expresiones (3.28) en el conjunto de colisiones, tenemos la desigualdad

$$
\frac{1}{2\left(1+\varepsilon_{i}\right)} p_{i}^{2}=h_{i}+\frac{1}{\sqrt{q^{2}+1 / 4}} .
$$

Sumando el término $0 \leq h_{j}+\frac{1}{\sqrt{q^{2}+1 / 4}}$ en el miembro del lado derecho obtenemos

$$
\frac{1}{2\left(1+\varepsilon_{i}\right)} p_{i}^{2} \leq h+\frac{2}{\sqrt{q^{2}+1 / 4}} .
$$


La proyección $\pi_{i}$ del conjunto anterior únicamente envía $q \mapsto q_{i}$, es decir que finalmente tenemos la restricción

$$
\frac{1}{2\left(1+\varepsilon_{i}\right)} p_{i}^{2} \leq h+\frac{2}{\sqrt{q_{i}^{2}+1 / 4}} .
$$

Las expresiones (3.33) y (3.35) indican que la curva de nivel $\Gamma_{i}$ está contenida en la región delimitada por la curva (3.31), y por lo tanto $\pi_{i}\left(\mathcal{C}_{x}\right)=\Gamma_{i}$. Lo que prueba el primer punto del lema.

2. Supongamos ahora que $h_{j}<h_{i}$. Por el punto anterior con desigualdad estricta, tenemos que $h_{j}^{2}>\left(\frac{h_{i}+h_{j}}{2}\right)^{2}$ de donde se obtiene

$$
\left|q_{i}\right| \leq \sqrt{\frac{1}{h_{j}^{2}}-\frac{1}{4}}<\sqrt{\frac{1}{\left(\frac{h_{i}+h_{j}}{2}\right)^{2}}-\frac{1}{4}} \leq \sqrt{\frac{1}{h_{i}^{2}}-\frac{1}{4}} .
$$

Las expresiones (3.28) deben cumplirse simultáneamente para $i$ y $j$ por lo que $q_{i}$ no alcanza sus valores máximo ni mínimo (considerada como una solución del problema de Sitnikov clásico) bajo la condición

$$
-\sqrt{\frac{1}{h_{j}^{2}}-\frac{1}{4}} \leq q_{i} \leq \sqrt{\frac{1}{h_{j}^{2}}-\frac{1}{4}} .
$$

Esto indica que $\pi_{i}\left(\mathcal{C}_{x}\right) \subset \Gamma_{i}$ pero $\Gamma_{i} \nsubseteq \pi_{i}\left(\mathcal{C}_{x}\right)$. Despejando $p_{i}$ de (3.34) y proyectando en $\mathcal{S}_{i}$ obtenemos

$$
p_{i}= \pm \sqrt{2\left(1+\varepsilon_{i}\right)} \sqrt{h_{i}+\frac{1}{\sqrt{q_{i}^{2}+1 / 4}}},
$$

y $\pi_{i}\left(\mathcal{C}_{x}\right)$ corresponderá a dos segmentos de $\Gamma_{i}$ uno para la raíz positiva de $p_{i}$ y otro para la raíz negativa bajo la condición (3.37).

En la Figura 3.6 se pueden ver las proyecciones $\pi_{i}\left(\mathcal{C}_{x}\right)$ para distintos valores de $x=\left(h_{3}, h_{4}\right)$ sobre el mismo nivel de energía $h=h_{3}+h_{4}$.

Teorema 3.21 (Jiménez-Pérez). El conjunto de colisiones $\mathcal{C}_{x}$ para cada fibra $\mathscr{T}_{x}=\mu^{-1}(x)$ corresponde a curvas parametrizadas por

$$
\left\{\begin{aligned}
q & =k \frac{\operatorname{sn}(\nu, k) \operatorname{dn}(\nu, k)}{2 \operatorname{dn}^{2}(\nu, k)-1} \\
p_{j} & =2 k \sqrt{2\left(1+\varepsilon_{j}\right)} \operatorname{cn}(\nu, k) \\
p_{i} & = \pm \sqrt{2\left(1+\varepsilon_{i}\right)} \sqrt{h_{i}-h_{j}+4 k^{2} \operatorname{cn}^{2}(\nu, k)}
\end{aligned}\right.
$$

donde $\varepsilon_{i}=(-1)^{i+1} \epsilon, k=\sqrt{2+h_{j}} / 2, h_{j} \leq h_{i}$ para $i, j=3,4 i \neq j$.

Demostración. El conjunto $\mathcal{C}_{x}$ está determinado por las ecuaciones (3.28). Por el Lema 3.9 sabemos que el valor de $q\left(=q_{i}=q_{j}\right)$ estará acotado 
por la condición (3.37), donde $h_{j}=\operatorname{mín}\left\{h_{3}, h_{4}\right\}$. Trabajemos con la ecuación correspondiente al subíndice $j$

$$
\frac{1}{2\left(1+\varepsilon_{j}\right)} p_{j}^{2}=h+\frac{1}{\sqrt{q^{2}+1 / 4}} .
$$

Como nuestro objetivo es parametrizar el conjunto, utilizaremos el cambio de variable $q=\frac{1}{2} \tan \theta$ y despejaremos $p_{j}$ del resultado obtenido. Esto nos dará

$$
\begin{aligned}
q & =\frac{1}{2} \tan \theta, \\
p_{j} & =\sqrt{2\left(1+\varepsilon_{i}\right)} \sqrt{h_{j}+2 \cos \theta},
\end{aligned}
$$

Considerando ahora $\theta=2 \phi$ y utilizando identidades trigonométricas obtenemos

$$
\begin{aligned}
q & =\frac{\sin \phi \cos \phi}{2 \cos ^{2} \phi-1}, \\
p_{j} & =\sqrt{2\left(1+\varepsilon_{j}\right)} \sqrt{h_{j}+2\left(1-2 \sin ^{2} \phi\right)}, \\
& =\sqrt{2\left(1+\varepsilon_{j}\right)} \frac{\sqrt{h_{j}+2}}{2} \sqrt{1-\frac{4}{h_{j}+2} \sin ^{2} \phi} .
\end{aligned}
$$

Finalmente, mediante el cambio de variable

$$
\sin \phi=\frac{\sqrt{h_{j}+2}}{2} \operatorname{sn}(\nu, k), \quad k=\frac{\sqrt{h_{j}+2}}{2},
$$

se obtiene

$$
\begin{aligned}
q & =k \frac{\operatorname{sn}(\nu, k) \operatorname{dn}(\nu, k)}{2 \operatorname{dn}^{2}(\nu, k)-1}, \\
p_{j} & =2 k \sqrt{2\left(1+\varepsilon_{j}\right)} \operatorname{cn}(\nu, k) .
\end{aligned}
$$

Esta es la solución al problema circular de Sitnikov si consideramos $q=q(t)$ para $t=t(\nu)$ dada en el Lema 3.7.

Ahora encontremos la expresión para el momento $p_{i}$. Por el Lemma 3.8 y dado que $h_{j} \leq h_{i}$ se cumple

$$
0 \leq h_{i}-h_{j}=\frac{1}{2\left(1+\varepsilon_{i}\right)} p_{i}^{2}-\frac{1}{2\left(1+\varepsilon_{j}\right)} p_{j}^{2} .
$$

Despejando $p_{i}$ obtenemos

$$
p_{i}= \pm \sqrt{2\left(1+\varepsilon_{i}\right)} \sqrt{h_{i}-h_{j}+\frac{1}{2\left(1+\varepsilon_{j}\right)} p_{j}^{2}}
$$

Sustituyendo $p_{j}$ en la expresión anterior tenemos

$$
p_{i}= \pm \sqrt{2\left(1+\varepsilon_{i}\right)} \sqrt{h_{i}-h_{j}+4 k^{2} \mathrm{cn}^{2}(\nu, k)} .
$$

La parametrización está dada por (3.40), (3.41) y (3.42) 


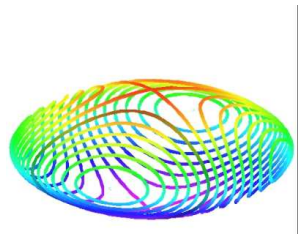

$h=-3$

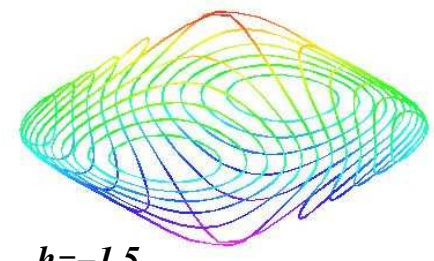

$h=-1.5$

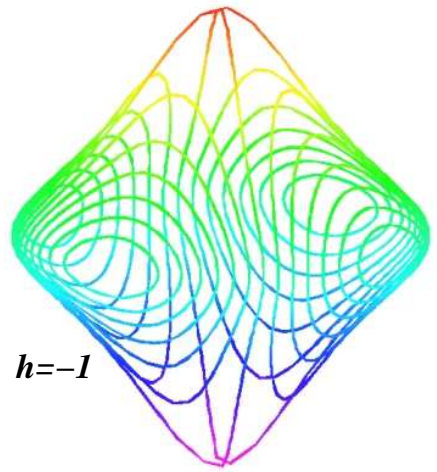

FiguRA 3.7. Intersección de las fibras $\mathscr{T}_{x}$ con el conjunto $\Delta$ para los niveles de energía $h=-3, h=-1,5$ y $h=-1$.

Un conjunto de estas curvas sobre las variedades de colisión para distintos valores de $H=h$ se pueden ver en la Figura 3.7 .

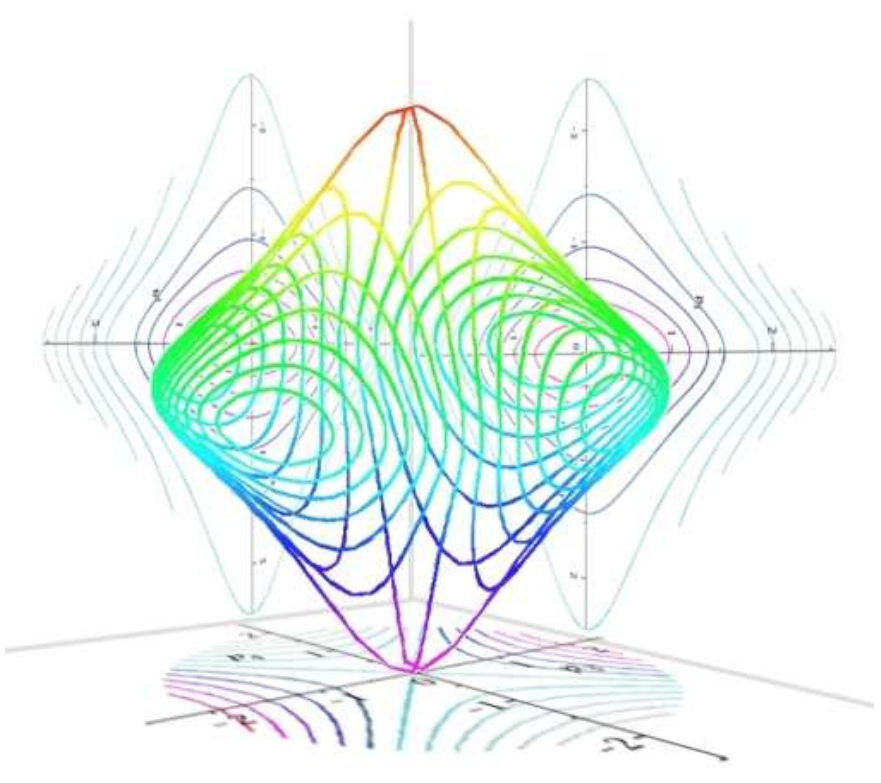

Figura 3.8. Proyecciones de las fibras $\mathscr{T}_{x}$ sobre los planos simplécticos $\mathcal{S}_{3}, \mathcal{S}_{4}$ y sobre el espacio de momentos $\left(p_{3}, p_{4}\right)$.

Corolario 1. El conjunto de colisiones $\mathcal{C}_{x}$ para cada toro invariante $\mathscr{T}_{x}=\mu^{-1}(x)$ corresponde a dos curvas simples cerradas, ajenas y simétricas si $h_{3} \neq h_{4}$. Para el caso $h_{3}=h_{4}$ este conjunto corresponde a dos curvas planas transversales que pertenecen a los planos $p_{4}= \pm \sqrt{\frac{1-\epsilon}{1+\epsilon}} p_{3}$.

Demostración. Cuando $h_{3} \neq h_{4}$, consideramos $h_{j}=\operatorname{mín}\left\{h_{3}, h_{4}\right\}$ y tendremos $h_{j}<h_{i}$. Consideremos nuevamente a $\Gamma_{j}=\left(q_{j}, p_{j}\right)$ como una 
curva de nivel del problema circular de Sitnikov clásico con energía $h_{j}$ sobre el subespacio simpléctico $\mathcal{S}_{j}$. Por el Lemma 3.9 se tiene que $\pi_{j}\left(\mathcal{C}_{x}\right)=\Gamma_{j}$ y por lo tanto $\mathcal{C}_{x}=\pi_{j}^{-1}\left(\Gamma_{j}\right)$ corresponde a un levantamiento de $\Gamma_{j}$ en la variedad de colisión con coordenadas

$$
\left(q_{j}, p_{j}\right) \mapsto\left(q_{j}, p_{j}, q_{j}, \pm \sqrt{2\left(1+\varepsilon_{i}\right)} \sqrt{h_{i}-h_{j}+\frac{1}{2\left(1+\varepsilon_{j}\right)} p_{j}^{2}}\right),
$$

Definamos las curvas $\gamma_{+}, \gamma_{-}: I \subset \mathbb{R} \rightarrow \Delta$ mediante

$$
\begin{aligned}
& \gamma_{+}(\nu)=\left(q, p_{j}, p_{i}^{+}\right) \\
& \gamma_{-}(\nu)=\left(q, p_{j}, p_{i}^{-}\right)
\end{aligned}
$$

donde $p_{i}^{+}$corresponde a la raíz positiva de $p_{i}$ y $p_{i}^{-}$corresponde a la raíz negativa. Entonces $\gamma_{+}$es simétrica a $\gamma_{-}$con respecto del plano $p_{i}=0$. Las parametrizaciones (3.40) y (3.41) nos indican que las curvas $\gamma_{+}$y $\gamma_{-}$son suaves, y serán cerradas si el denominador de (3.40) no se anula.

El mínimo valor que puede tomar la función $\operatorname{dn}(\nu, k)$ con argumento real es cuando $\nu=K$ (véase el Apéndice C). Utilizando la identidad (C.33) tenemos sucesivamente que

$$
\begin{aligned}
2 \operatorname{dn}^{2}(K, k)-1 & =2\left(k^{\prime}\right)^{2}-1 \\
& =2\left(1-k^{2}\right)-1 \\
& =1-\frac{2+h_{j}}{2} \\
& =-h_{j} / 2 \\
& >0
\end{aligned}
$$

Finalmente, el radicando de (3.42) siempre es positivo ya que $h_{j}<h_{i}$, esto indica que $p_{i}^{+}>0$ y $p_{i}^{-}<0$ para toda $\nu \in \mathbb{R}$, y podemos concluir que las curvas son ajenas.

En el caso en que $h_{i}=h_{j}$ la expresión para $p_{i}$ se reduce a

$$
p_{i}=2 k \sqrt{2\left(1+\varepsilon_{i}\right)} \operatorname{cn}(\nu, k) .
$$

La primera parte del Lema 3.8 nos afirma que la imagen de las curvas $\gamma_{+} \mathrm{y}$ $\gamma_{-}$debe satisfacer

$$
\left(\frac{1}{\sqrt{2(1+\epsilon)}} p_{3}-\frac{1}{\sqrt{2(1-\epsilon)}} p_{4}\right)\left(\frac{1}{\sqrt{2(1+\epsilon)}} p_{3}+\frac{1}{\sqrt{2(1-\epsilon)}} p_{4}\right)=0
$$

y por lo tanto estará sobre los planos $p_{4}= \pm \sqrt{\frac{1-\epsilon}{1+\epsilon}} p_{3}$.

Corolario 2. Consideremos que $i=4$ y $j=3$, es decir que $h_{3}<h_{4}$. Entonces la curva cerrada simple $\gamma_{+}$corresponde a colisiones mientras que la curva $\gamma_{-}$corresponde expulsiones. Cuando $h_{4}<h_{3}$ el comportamiento de $\gamma_{+} y \gamma_{-}$se invierte. 
Demostración. Sea $h_{3}<h_{4}$, entonces se obtiene que

$$
0<h_{4}-h_{3}=\frac{1}{2(1-\epsilon)} p_{4}^{2}-\frac{1}{2(1+\epsilon)} p_{3}^{2},
$$

de donde $\pm p_{4}>\sqrt{\frac{1-\epsilon}{1+\epsilon}}\left|p_{3}\right| \cdot \gamma_{+}$corresponde al valor positivo de $p_{4}$ es decir $p_{4}>\sqrt{\frac{1-\epsilon}{1+\epsilon}}\left|p_{3}\right|$ mientras que $\gamma_{-}$corresponde al valor negativo. Considerando que $p_{3}=(1+\epsilon) \dot{q}_{3}$ y $p_{4}=(1-\epsilon) \dot{q}_{4}$ se obtiene que

$$
\sqrt{\frac{1+\epsilon}{1-\epsilon}}\left|\dot{q}_{3}\right|-\dot{q}_{4} \sim\left|\dot{q}_{3}\right|-\dot{q}_{4}<0
$$

Consideremos el valor positivo para $\dot{q}_{3}$. Por lo tanto, para $\epsilon$ suficientemente pequeña tenemos que la distancia $q_{3}-q_{4}$ va decreciendo con el tiempo y por hipótesis solo consideramos el semiespacio $q_{3}>q_{4}$, entonces la curva $\gamma_{+}$corresponde a colisión. De manera análoga se tiene que para la raíz negativa $-p_{4}>\sqrt{\frac{1-\epsilon}{1+\epsilon}}\left|p_{3}\right|$, considerando el valor negativo de $p_{3}$ se cumplirá la desigualdad

$$
\sqrt{\frac{1+\epsilon}{1-\epsilon}} \dot{q}_{3}-\dot{q}_{4} \sim \dot{q}_{3}-\dot{q}_{4}>0 .
$$

Esto indica que los cuerpos se están separando y por lo tanto $\gamma_{-}$corresponde a eyecciones.

Por otro lado, si $h_{4}<h_{3}$ se intercambian $p_{3}$ y $p_{4}$ en las desigualdades, pero la hipótesis que $q_{3}>q_{4}$ establecerá el intercambio de comportamiento entre $\gamma_{+}$que correspondera a eyecciones y $\gamma_{-}$que serán colisiones.

Nota 3.8. La parametrización del Teorema [3.21 se puede extender a una parametrización $\psi(h, \nu): U \subset \mathbb{R}^{2} \rightarrow \Delta$ de la variedad de colisión para cada $h>-4$ fija donde $U$ es la región bajo la gráfica de la función $f(x)=4 K\left(\frac{\sqrt{4+x}}{2}\right)$ y con $x \in(-4, h)$. Dicha parametrización se realizará en un trabajo futuro.

\subsection{TRANSFERENCIA DE MOMENTO.}

La regularización nos permite continuar las soluciones de manera analítica al conjunto $\Delta$. Sin embargo, cuando las masas infinitesimales de los secundarios son diferentes $m_{3} \neq m_{4}$, debemos dar algunas condiciones adicionales para extender las soluciones más allá de la singularidad. Esto es consecuencia inmediata de las condiciones para tener rebote elástico y de la conservación del momento lineal en el instante de la colisión. A estos fenómenos se les conoce como transferencia de energía y transferencia de momento 41 .

Iniciemos con definir la notación que utilizaremos a lo largo de esta sección. Un acento circunflejo ( ` ) sobre una variable, denotará el valor de esa variable después de una colisión. La letra $v_{i}$ denotará la velocidad de la $i$-ésima partícula y se introduce para evitar que la notación $\hat{\dot{q}}_{i}$ sea cansada. 
Por lo tanto, $v_{3}$ y $p_{3}$ corresponden a la velocidad y al momento de la partícula con posición $q_{3}$ antes de una colisión, mientras que $\hat{v}_{3}$ y $\hat{p}_{3}$ corresponden a la velocidad y al momento de la misma partícula después de una colisión.

En mecánica de particulas, se utiliza la ecuación

$$
\hat{v}_{i}-\hat{v}_{j}=-\delta\left(v_{i}-v_{j}\right), \quad i \neq j,
$$

para determinar la velocidad relativa de dos partículas después de una colisión. El término $\delta \in[0,1]$ se conoce como el coeficiente de restitución y determina la conservación de energía cinética durante una colisión.

Definición 3.3. Si el coeficiente de restitución es $\delta=1$ se dice que se tiene un rebote elástico durante la colisión. Si $\delta<1$ se dice que se tiene un rebote inelástico. Para el caso extremo $\delta=0$ se dice que se tiene un rebote totalmente inelástico.

Un rebote elástico implica que no habrá pérdida de la energía cinética durante una colisión, mientras que en un rebote inelástico si existirá pérdida de energía cinética. En particular, un sistema conservativo con colisiones tendrá un coeficiente de restitución $\delta=1$.

La condición de rebote elástico en nuestro sistema se escribe como

$$
\hat{v}_{3}-\hat{v}_{4}=-\left(v_{3}-v_{4}\right) \text {. }
$$

Adicionalmente, uno de los invariantes principales de un sistema mecánico con colisiones es el momento lineal

$$
\hat{p}_{3}+\hat{p}_{4}=p_{3}+p_{4},
$$

que es independiente de la existencia de fuerzas externas (véase el capítulo 3 de (41). Por medio de las relaciones (3.43) y (3.44) vamos a determinar el intercambio de momento entre los secundarios del problema circular de Sitnikov cuando pasan a través de una colisión.

Lema 3.10 (Jiménez-Pérez). En el problema circular de Sitnikov de 2+2 cuerpos, el intercambio de momento entre los secundarios al instante de una colisión está dado por $\hat{\mathbf{p}}=$ Ap donde $\mathbf{p}=\left(p_{3}, p_{4}\right)^{T} y$

$$
A=\left(\begin{array}{cc}
\epsilon & 1+\epsilon \\
1-\epsilon & -\epsilon
\end{array}\right) .
$$

Demostración. Utilizando la relación entre momentos y velocidades dada en (2.13), podemos escribir la expresión (3.43) en términos de momentos de la forma

$$
\frac{1}{(1+\epsilon)} \hat{p}_{3}-\frac{1}{(1-\epsilon)} \hat{p}_{4}=-\left(\frac{1}{(1+\epsilon)} p_{3}-\frac{1}{(1-\epsilon)} p_{4}\right),
$$

Resolviendo de manera simultánea (3.44) y (3.46), para $\hat{p}_{3}$ y $\hat{p}_{4}$ obtenemos

$$
\begin{aligned}
& \hat{p}_{3}=\epsilon p_{3}+(1+\epsilon) p_{4} \\
& \hat{p}_{4}=(1-\epsilon) p_{3}-\epsilon p_{4} .
\end{aligned}
$$


Matricialmente tenemos $\hat{\mathbf{p}}=A \mathbf{p}$ donde $\mathbf{p}=\left(p_{3}, p_{4}\right)^{T} \mathrm{y}$

$$
A=\left(\begin{array}{cc}
\epsilon & 1+\epsilon \\
1-\epsilon & -\epsilon
\end{array}\right) \text {. }
$$

Diremos que $A$ es la matriz de transición en las colisiones. En el espacio tangente $T_{\mathbf{q}} \mathbb{R}^{2}$ el sistema se escribe como $\hat{\mathbf{v}}=A^{T} \mathbf{v}$. Ahora que establecimos el intercambio de momento entre los secundarios durante una colisión, podemos enunciar el siguiente resultado

LEMA 3.11 (Jiménez-Pérez). El intercambio de energía entre los secundarios está dado por la relación

$$
\begin{aligned}
& \hat{h}_{3}=h_{4}+\epsilon^{2}\left(\frac{1}{2(1+\epsilon)} p_{3}^{2}-\frac{1}{2(1-\epsilon)} p_{4}^{2}\right)+\epsilon p_{3} p_{4}, \\
& \hat{h}_{4}=h_{3}-\epsilon^{2}\left(\frac{1}{2(1+\epsilon)} p_{3}^{2}-\frac{1}{2(1-\epsilon)} p_{4}^{2}\right)-\epsilon p_{3} p_{4} .
\end{aligned}
$$

Demostración. Primero veamos que

$$
\begin{aligned}
& \hat{h}_{3}=\frac{1}{2(1+\epsilon)} \hat{p}_{3}^{2}-\frac{1}{\sqrt{q_{0}^{2}+1 / 4}}, \\
& \hat{h}_{4}=\frac{1}{2(1-\epsilon)} \hat{p}_{4}^{2}-\frac{1}{\sqrt{q_{0}^{2}+1 / 4}} .
\end{aligned}
$$

Considerando el lema anterior, tenemos que

$$
\begin{aligned}
& \hat{h}_{3}=\frac{1}{2(1+\epsilon)}\left(\epsilon p_{3}+(1+\epsilon) p_{4}\right)^{2}-\frac{1}{\sqrt{q_{0}^{2}+1 / 4}}, \\
& \hat{h}_{4}=\frac{1}{2(1+\epsilon)}\left((1-\epsilon) p_{3}-\epsilon p_{4}\right)^{2}-\frac{1}{\sqrt{q_{0}^{2}+1 / 4}} .
\end{aligned}
$$

Desarrollando el cuadrado en la ecuación para $\hat{h}_{3}$ tenemos sucesivamente

$$
\begin{aligned}
\hat{h}_{3} & =\frac{\epsilon^{2}}{2(1+\epsilon)} p_{3}^{2}+\epsilon p_{3} p_{4}+\frac{1+\epsilon}{2} p_{4}^{2}-\frac{1}{\sqrt{q_{0}^{2}+1 / 4}}, \\
& =\frac{\epsilon^{2}}{2(1+\epsilon)} p_{3}^{2}+\epsilon p_{3} p_{4}+\frac{1-\epsilon^{2}}{2(1-\epsilon)} p_{4}^{2}-\frac{1}{\sqrt{q_{0}^{2}+1 / 4}}, \\
& =\left(\frac{1}{2(1-\epsilon)} p_{4}^{2}-\frac{1}{\sqrt{q_{0}^{2}+1 / 4}}\right)+\frac{\epsilon^{2}}{2(1+\epsilon)} p_{3}^{2}+\epsilon p_{3} p_{4}-\frac{\epsilon^{2}}{2(1-\epsilon)} p_{4}^{2}, \\
& =h_{4}+\epsilon^{2}\left(\frac{1}{2(1+\epsilon)} p_{3}^{2}-\frac{1}{2(1-\epsilon)} p_{4}^{2}\right)+\epsilon p_{3} p_{4},
\end{aligned}
$$

Resolviendo de manera análoga para el valor de $\hat{h}_{4}$ obtenemos el resultado deseado. 
LEMA 3.12 (Jiménez-Pérez). En el problema restringido circular de Sitnikov con $2+2$ cuerpos las soluciones que parten de un toro invariante, se preservan en dicho toro a través de las colisiones si y sólo si la colisión se da en el plano $p_{3}+p_{4}=0$.

Demostración. Supongamos primero que $p_{3}+p_{4}=0$. Cada toro invariante $\mathscr{T}_{x}=\mu^{-1}(x)$ está determinado de manera única por el valor de $x \in \mathfrak{g}^{*}$, con $x=\left(h_{3}, h_{4}\right)$. La solución se preserva en el mismo toro invariante si $\left(\hat{h}_{3}, \hat{h}_{4}\right)=\left(h_{3}, h_{4}\right)$. Utilizando la primera expresión del Lema 3.11 para $\hat{h}_{3}=h_{3}$ obtenemos

$$
\begin{aligned}
h_{3} & =h_{4}+\epsilon^{2}\left(\frac{1}{2(1+\epsilon)} p_{3}^{2}-\frac{1}{2(1-\epsilon)} p_{4}^{2}\right)+\epsilon p_{3} p_{4}, \\
h_{3}-h_{4} & =\epsilon^{2}\left(\frac{1}{2(1+\epsilon)} p_{3}^{2}-\frac{1}{2(1-\epsilon)} p_{4}^{2}\right)+\epsilon p_{3} p_{4} .
\end{aligned}
$$

La ecuación (3.29) del Lema 3.8 reduce la condición anterior a

$$
\left(1-\epsilon^{2}\right)\left(\frac{1}{2(1+\epsilon)} p_{3}^{2}-\frac{1}{2(1-\epsilon)} p_{4}^{2}\right)=\epsilon p_{3} p_{4},
$$

y finalmente tenemos

$$
\frac{1-\epsilon}{2} p_{3}^{2}-\epsilon p_{3} p_{4}-\frac{1+\epsilon}{2} p_{4}^{2}=0 .
$$

Resolviendo para $p_{3}$ por la fórmula general de segundo grado tenemos las dos soluciones

$$
p_{3}=-p_{4} \quad \text { y } \quad p_{3}=\frac{1+\epsilon}{1-\epsilon} p_{4} .
$$

La segunda solución implica que

$$
\dot{q}_{3}=\frac{1}{1+\epsilon} p_{3}=\frac{1}{1-\epsilon} p_{4}=\dot{q}_{4}
$$

Es decir, ambos cuerpos tienen la misma velocidad y la misma posición en la colisión y corresponde a la única solución $\sigma(t)$ que se encuentra sobre la variedad de colisión $\Sigma_{h} \cap \Delta$ para toda $t \in \mathbb{R}$, lo que es físicamente imposible. Por lo tanto, la única solución posible es que al instante de la colisión el momento lineal sea nulo $p_{3}+p_{4}=0$.

Este mismo resultado se obtiene si consideramos que para preservar las energías relativas $\hat{h}_{3}=h_{3}$ y $\hat{h}_{4}=h_{4}$ se deben preservar los momentos relativos, es decir que $\hat{p}_{3}^{2}=p_{3}^{2}$ y $\hat{p}_{4}^{2}=p_{4}^{2}$. Como la igualdad $\hat{p}_{i}=p_{i}$ no es posible, ya que en la colisión existe un rebote, se requiere que $\hat{p}_{i}=-p_{i}$ para $i=3,4$. Utilizando este hecho y la condición que $\hat{\mathbf{p}}=A \mathbf{p}$ tenemos el sistema

$$
\begin{aligned}
& -p_{3}=\epsilon p_{3}+(1+\epsilon) p_{4} \\
& -p_{4}=(1-\epsilon) p_{3}-\epsilon p_{4}
\end{aligned}
$$




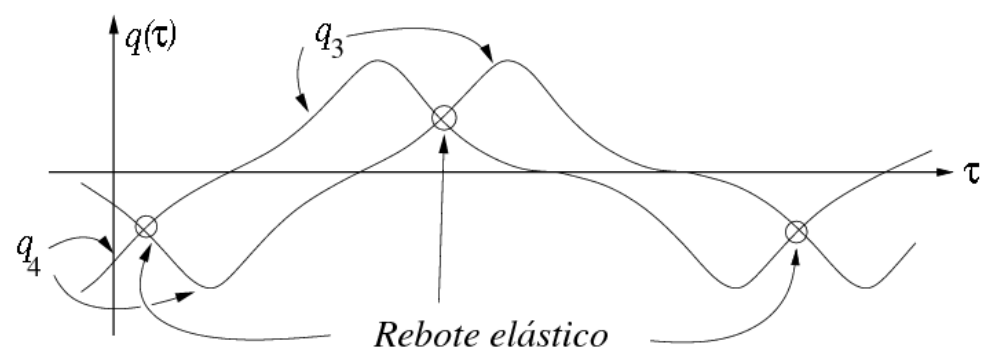

FigurA 3.9. Soluciones a través de las colisiones.

cuya solución es $p_{3}+p_{4}=0$. Sin embargo, la solución $p_{3}=\frac{1+\epsilon}{1-\epsilon} p_{4}$ no aparece específicamente mediante este análisis, por lo que la resolución mediante el Lema 3.11 es la más adecuada.

Proposición 3.20 (Jiménez-Pérez, Lacomba). En el problema circular restringido de Sitnikov de $2+2$ cuerpos, con función Hamiltoniana

$$
H=\frac{1}{2(1+\epsilon)} p_{3}^{2}+\frac{1}{2(1-\epsilon)} p_{4}^{2}-\frac{1+\epsilon}{\sqrt{q_{3}^{2}+1 / 4}}-\frac{1-\epsilon}{\sqrt{q_{4}^{2}+1 / 4}}
$$

el flujo $\varphi_{t}(x)$ puede extenderse a un flujo completo cuando $\epsilon=0$. La extensión se realiza de manera natural considerando que los cuerpos se atraviesan en las colisiones en vez de considerar rebote elástico, mediante la simetría

$$
\left(q_{3}, q_{4}, p_{3}, p_{4}, t\right) \mapsto\left(q_{4}, q_{3}, p_{4}, p_{3}, t\right),
$$

que extiende el sistema Hamiltoniano a todo el espacio fase.

Demostración. Al considerar que los cuerpos se atraviesan durante la colisión se debe aplicar la simetría $\left(q_{3}, q_{4}, p_{3}, p_{4}, t\right) \mapsto\left(q_{4}, q_{3}, p_{4}, p_{3}, t\right)$, y las condiciones de transición $\hat{p}_{3}=p_{4}, \hat{p}_{4}=p_{3}, \hat{v}_{3}=v_{4}, \mathrm{y} \hat{v}_{4}=v_{3}$. Por lo tanto se tiene

$$
A=A^{T}=\left(\begin{array}{ll}
0 & 1 \\
1 & 0
\end{array}\right)
$$

que se cumple si y sólo si $\epsilon=0$. Consecuentemente, la singularidad es imperceptible para los secundarios.

La conversa de este resultado también se cumple, sin embargo necesitaremos algunos resultados adicionales que detallaremos en el siguiente capítulo.

Como vimos en la proposición anterior, si $\epsilon=0\left(m_{3}=m_{4}\right)$ las soluciones se extienden de manera suave si se considera que los cuerpos se atraviesan en las colisiones. Por otro lado, si $p_{3}+p_{4}=0$ tenemos que $p_{3}=-p_{4}$ y por lo tanto $\hat{p}_{3}=-p_{3}$ y $\hat{p}_{4}=-p_{4}$. Esto nos indica que la solución es reversible a partir de la colisión (véase la Figura 3.9).

Este hecho sugiere un especial interés en las soluciones que llegan al plano $\mathcal{P} \subset \Delta$ de dimensión 2 definido por

$$
\mathcal{P}:=\left\{\left(q_{3}, q_{4}, p_{3}, p_{4}\right) \in \Delta \mid p_{3}=-p_{4}\right\} .
$$


Las únicas soluciones que podemos extender a través de las colisiones y que preserven el momento y la energía relativa de cada cuerpo son las que llegan y parten de $\mathcal{P}$.

Esto nos permite clasificar las soluciónes en los casos siguientes

1. Si $\epsilon=0$ entonces las masas infinitesimales de los secundarios son iguales $m_{3}=m_{4}$ y las soluciones cambian de toro $\mathscr{T}_{x} \mapsto \mathscr{T}_{x^{\circ}}$ al toro simétrico asociado al punto $x^{\circ}=\left(h_{4}, h_{3}\right)$. Mediante la simetría $\left(q_{3}, q_{4}, p_{3}, p_{4}, t\right) \mapsto\left(q_{4}, q_{3}, p_{4}, p_{3}, t\right)$, se puede considerar que están en el mismo toro.

2. Si las masas infinitesimales son distintas $m_{3} \neq m_{4}$ entonces las soluciones permanecen en el mismo toro si y sólo si pasan por el plano $\mathcal{P}$.

Para estudiar las órbitas periódicas a través de colisión, tendremos que estudiar los dos casos anteriores. En particular, para el primer caso no se requiere conocer el instante en que ocurren las colisiones, ya que no existe ningún cambio en las órbitas además de la simetría

$$
\left(q_{3}, q_{4}, p_{3}, p_{4}, t\right) \mapsto\left(q_{4}, q_{3}, p_{4}, p_{3}, t\right) .
$$

Este caso lo estudiaremos en lo que resta de este capítulo.

Por otra parte, la situación donde $\epsilon>0$ cambia completamente ya que el flujo no se puede extender a un flujo completo. En este caso las soluciones que generan órbitas periódicas a través de colisión deben ser buscadas de una manera muy particular. Las órbitas periódicas que se preservan en ese caso serán estudiadas en el capítulo siguiente.

\subsection{SOLUCIONES PERIÓDICAS EN EL PROBLEMA CIRCULAR: $m_{3}=m_{4}$.}

Cuando las masas de los cuerpos infinitesimales son iguales $m_{3}=m_{4}$, el parámetro que aparece en la función Hamiltoniana (3.1) se anula, y dicha función se convierte en

$$
H=\left(\frac{1}{2} p_{3}^{2}-\frac{1}{\sqrt{q_{3}^{2}+1 / 4}}\right)+\left(\frac{1}{2} p_{4}^{2}-\frac{1}{\sqrt{q_{4}^{2}+1 / 4}}\right) .
$$

En este caso las soluciones del problema circular de Sitnikov con $2+2$ cuerpos tiene la forma

$$
\phi(t)=\left(k_{3} \frac{s_{3} d_{3}}{1-2 k_{3}^{2} s_{3}^{2}}, 2 \sqrt{2} k_{3} c_{3}, k_{4} \frac{s_{4} d_{4}}{1-2 k_{4}^{2} s_{4}^{2}}, 2 \sqrt{2} k_{4} c_{4}\right),
$$

donde $s_{i}=\operatorname{sn}\left(\nu_{i}, k_{i}\right), c_{i}=\operatorname{cn}\left(\nu_{i}, k_{i}\right)$ y $d_{i}=\operatorname{dn}\left(\nu_{i}, k_{i}\right)$, son las funciones

elípticas de Jacobi, $k_{i}=\frac{\sqrt{2+h_{i}}}{2}$ y $\nu_{i}=\nu_{i}\left(t, k_{i}\right)$. Como $\epsilon=0$, los valores de los módulos son equivalentes para ambos secundarios.

Cuando los valores de la aplicación momento se encuentran en el abierto $(-2,0) \times(-2,0)$, la evolución del sistema es acotada. En esta región es posible tener órbitas periódicas pasando por colisión bajo condiciones específicas. 
Necesitaremos algunas propiedades adicionales acerca de la función del periodo $T(k)=T(h)$, donde $k=k(h)$ mediante la relación $k(h)=\frac{\sqrt{2+h}}{2}$. Dichas propiedades se resumen en el siguiente resultado:

Teorema 3.22 (Belbruno, Ollé, Llibre 1994). Sea $T(k)=T(h)$ el periodo de la solución del problema circular de Sitnikov con energía $h$; entonces las siguientes afirmaciones se cumplen

1. $\lim _{h \rightarrow-2^{+}} T(h)=\frac{\pi}{\sqrt{2}}$,

2. $\lim _{h \rightarrow 0^{-}} T(h)=\infty$,

3. $\frac{d T}{d h}>0, \quad \forall h \in(-2,0)$,

4. $\lim _{h \rightarrow-2^{+}} \frac{d T}{d h}=\frac{\pi(1+4 \sqrt{2})}{16}$,

5. $\lim _{h \rightarrow 0^{-}} \frac{d T}{d h}=\infty$.

La demostración de este teorema se obtiene de manera directa a partir de la definición del periodo $T(k(h))=T(h)$ como función de $h$. Este resultado se estableció en el Lema 6 y la demostración puede revisarse en 8 .

Por supuesto, $T(h)$ es continua en el dominio $h \in(-2,0)$, y el Teorema 3.22 asegura la existencia de órbitas periódicas para el problema de Sitnikov clásico con periodo $T$ para toda $T>\frac{\pi}{\sqrt{2}}$. Ahora debemos considerar las órbitas periódicas del sistema con 3 cuerpos (considerando aún en el problema de Sitnikov clásico). Para ello es necesario que $T=\frac{2 \pi p}{q}$ con $p, q \in \mathbb{N}$ coprimos, ya que el periodo de los primarios es $2 \pi$.

Nota 3.9. Dados $l$ números naturales $\left\{n_{1}, n_{2}, \ldots, n_{l}\right\}$ usaremos la notación $\left(n_{1}, n_{2}, \ldots, n_{l}\right)=m$ para indicar que $m \in \mathbb{N}$ es el máximo común divisor de $\left\{n_{1}, n_{2}, \ldots, n_{l}\right\}$.

En particular, si $p, q, n \in \mathbb{N}$ son tres números naturales arbitrarios, entonces $(p, q, n)=1$ indica que dichos números no tienen factores en común.

Proposición 3.21 (Corbera, Llibre 2002). Dados $p, q \in \mathbb{N}$ con $p>\frac{q}{2 \sqrt{2}}$ $y(p, q)=1$ existe una única $\hat{h} \in(-2,0)$ tal que la solución de problema circular de Sitnikov con condiciones iniciales

$$
q(0)=q_{0}, \quad p(0)= \pm \sqrt{2\left(\hat{h}+\frac{1}{\sqrt{q_{0}^{2}+\frac{1}{4}}}\right)}
$$

es una solución periódica de periodo $T=\frac{2 \pi p}{q}$.

La demostración se puede revisar en $\mathbf{2 5}$.

Cuando estudiamos el problema restringido doble de Sitnikov como dos problemas de Sitnikov desacoplados, podemos ver que las soluciones periódicas se obtienen mediante dos soluciones periódicas del problema clásico de Sitnikov. 
El cuerpo con posición $q_{i}$ tendrá tiempo de retorno $\nu_{i}=4 K\left(k_{i}\right)$ en el tiempo reescalado y $t(4 K)=T\left(k_{i}\right)$ o $T\left(h_{i}\right)=t\left(4 K\left(k\left(h_{i}\right)\right)\right)-t(0)$ en el tiempo real $t=t\left(\nu_{i}\right)$, para $i=3,4$, es decir

$$
T\left(k\left(h_{i}\right)\right)=\frac{1}{\sqrt{2}\left(1-2 k_{i}^{2}\right)}\left[2 E\left(k_{i}\right)-K\left(k_{i}\right)+\Pi\left(2 k_{i}^{2}, k_{i}\right)\right]
$$

donde $k_{i}=\frac{\sqrt{2+h_{i}}}{2}$ y $K\left(k_{i}\right), E\left(k_{i}\right)$, y $\Pi\left(2 k_{i}^{2}, k_{i}\right)$ son las integrales elípticas completas de primera, segunda y tercera especie respectivamente (véase el Apéndice (C).

Con estos elementos, caracterizaremos las órbitas periódicas del problema circular doble de Sitnikov.

A continuación daremos algunas definiciones y estableceremos las condiciones que producirán órbitas periódicas en nuestro sistema.

Definición 3.4. Decimos que $\varphi(t)$ es una solución periódica de periodo $\tau$ con $\tau>0$ si $\varphi(t+\tau)=\varphi(t)$ para toda $t \in \mathbb{R}$ y no existe $\hat{\tau} \in(0, \tau)$ tal que $\varphi(t+\hat{\tau})=\varphi(t)$, i.e., $\tau$ es el periodo mínimo.

Proposición 3.22 (Jiménez-Pérez). Para cada solución periódica del problema circular doble de Sitnikov existen ternas $(p, q, n) \in \mathbb{Z}^{3}$ tal que $(p, q, n)=1$, donde se cumple que $p>\frac{q}{2 \sqrt{2}}$ y $p>\frac{n}{2 \sqrt{2}}$. Los periodos de dichas soluciones están relacionados a las energías parciales mediante

$$
\tau=2 p \pi=q T\left(h_{1}\right)=n T\left(h_{2}\right)
$$

Nota 3.10. Las parejas $(p, q)$ y $(p, n)$ no son necesariamente coprimos, sin embargo, alguna de las tres combinaciones $(p, q),(p, n),(q, n)$ debe ser coprimos para asegurar que $(p, q, n)=1$.

Nota 3.11. La superficie de energía del problema circular doble de Sitnikov que acepta la solución periódica con periodo $\tau=2 \pi=T\left(h_{3}\right)=T\left(h_{4}\right)$ es una superficie no compacta. El valor de $T(h)$ en $h=-1$ es

$$
T(-1)=\sqrt{2}\left(2 E\left(\frac{1}{2}\right)-F\left(\frac{1}{2}\right)+\Pi\left(\frac{1}{2}, \frac{1}{2}\right)\right) .
$$

La estimación numérica de este valor es

$$
\frac{T(-1)}{2 \pi}=0.824429907123718<1 .
$$

Como $T(h)$ es una función creciente de $h$ entonces $T\left(h_{i}\right)=2 \pi$ se obtiene para $h_{i}>-1 y-2<h_{3}+h_{4}$, por lo tanto $\Sigma=H^{-1}\left(h_{3}+h_{4}\right)$ es no compacta (véase la Figura [3.3).

Definición 3.5. Decimos que una superficie de energía $\Sigma=H^{-1}(h)$ acepta una solución periódica si existe una terna $(p, q, n) \in \mathbb{N}^{3}$ con las siguientes propiedades:

P1. $(p, q, n)=1$,

P2. $p>\frac{q}{2 \sqrt{2}}, p>\frac{n}{2 \sqrt{2}}$ 
tal que

$$
\Sigma=H^{-1}\left(T^{-1}\left(\frac{p}{q} 2 \pi\right)+T^{-1}\left(\frac{p}{n} 2 \pi\right)\right) .
$$

Escribiremos $\Sigma_{h}=H^{-1}(h)$ para indicar la dependencia con respecto a $h$.

Denotaremos al conjunto de superficies de energía que aceptan órbitas periódicas mediante

$$
\mathfrak{M}=\left\{\Sigma=H^{-1}\left(h_{*}\right) \mid h_{*}=T^{-1}\left(\frac{p}{q} 2 \pi\right)+T^{-1}\left(\frac{r}{s} 2 \pi\right), P 1, P 2 \text { se cumplen }\right\} .
$$

Teorema 3.23 (Jiménez-Pérez). En el problema circular doble de Sitnikov, el conjunto de superficies de nivel $\Sigma \in \mathfrak{M}$ que contienen toros foliados por órbitas periódicas es numerable. Además, el conjunto de valores $h_{*} \in H\left(T^{*} \mathbb{R}^{2}\right) \subset \mathbb{R}$ tal que $\Sigma_{h_{*}} \in \mathfrak{M}$, es denso en $(-4,0)$ y tiene medida cero en $\mathbb{R}$.

En la literatura, se tiene bien documentado el hecho que los toros resonantes forman un conjunto denso en la imagen de la aplicación momento. Sin embargo, Pugh y Robinson probaron en 1983 [86 que de manera genérica, las órbitas periódicas de los sistemas Hamiltonianos son densas en un subconjunto abierto contenido en la unión de las superficies de energía compactas y regulares. Además, afirman que utilizando un argumento de Fubini, este resultado se aplica a cualquier superficie de energía compacta y regular.

En contraste, el teorema anterior asegura que existe un conjunto de valores para $h_{*} \in \mathbb{R}$ de medida total, tal que $\Sigma_{h_{*}} \notin \mathfrak{M}$. Este es el comportamiento genérico de los sistemas Hamiltonianos separables completamente integrables.

La demostración de este teorema se sigue de inmediato de los siguientes dos lemas

Lema 3.13. Para cada $N \in \mathbb{N}$ el problema circular doble de Sitnikov tiene soluciones periódicas de periodo $2 N \pi$

Demostración. Basta con exhibir al menos una solución periódica de periodo $\tau=2 N \pi$. Esto es inmediato del hecho que estas soluciones existen en el problema circular de Sitnikov ( $2+1$ cuerpos).

Para cada $N \in \mathbb{N}$ se puede elegir como terna, la combinación $p=N$ y $q=n=1$ que producirá

$$
(p, q)=1 \quad \text { and } \quad(p, n)=1
$$

con

$$
p>\frac{q}{2 \sqrt{2}} \text { and } \quad p>\frac{n}{2 \sqrt{2}}
$$


utilizando la Proposición 2.8 en [25] sabemos que existen valores $h_{3}, h_{4} \in$ $(-2,0)$ tal que

$$
T\left(h_{3}\right)=\frac{2 \pi p}{q} \quad \text { and } \quad T\left(h_{4}\right)=\frac{2 \pi p}{n}
$$

y por lo tanto la superficie de energía $\Sigma_{h_{3}+h_{4}}=H^{-1}\left(h_{3}+h_{4}\right)$ contiene un toro resonante foliado por una familia de órbitas periódicas de periodo

$$
\tau=2 \pi N=T\left(h_{3}\right)=T\left(h_{4}\right)
$$

Antes de enunciar el segundo lema necesario para la demostración del teorema debemos definir la función $\varphi$ de Euler, que se utiliza en la teoría de números.

Definición 3.6. Se define la función totient o función $\varphi$ de Euler $\varphi(p)$ de un número entero $p$ como

$$
\varphi(p)=p \prod_{n \mid p}\left(1-\frac{1}{n}\right)
$$

donde el producto incluye a todos los enteros $n$ que son coprimos de $p$. Dicha función representa el número de enteros positivos menores o iguales a $p$ que son coprimos con $p$.

El siguiente lema se refiere a la finitud de toros resonantes foliados con órbitas periódicas con un periodo predeterminado $\tau$.

Lema 3.14. Para cada $N \in \mathbb{N}$ fija, el problema circular doble de Sitnikov tiene un número finito de toros resonantes foliados por órbitas periódicas con periodo $\tau=2 N \pi$. El número

$$
8 N \varphi(N)+\sum_{\substack{q<2 \sqrt{2} N,(N, q) \neq 1}} \varphi(q)
$$

es una cota superior (aunque no es una cota óptima).

Demostración. Para cada $N \in \mathbb{N}$ fija, existen ternas $(N, q, n) \in \mathbb{N}^{3}$, que satisfacen las propiedades P1 y P2 de la Definición 3.5. Por lo tanto, buscamos el número $C_{N}$ de ternas que no tengan factores en común, es decir que $(N, q, n)=1$. Es fácil ver que para cada $q<2 \sqrt{2} N$ y $(N, n)=1$, la terna $(N, q, n)$ no tiene divisores comunes. Estas ternas son exactamente $(2 \sqrt{2} N) \cdot(2 \sqrt{2} \varphi(N))=8 N \varphi(N)$.

Adicionalmente, debemos agregar todas las parejas $(q, n)$ de números coprimos tales que $(N, q)$ y $(N, n)$ no sean coprimos. Esto significa que para cada entero $q<2 \sqrt{2} N$ con $(N, q) \neq 1$ debemos agregar el número de 
coprimos $\varphi(q)$. Entonces tenemos

$$
C_{N}<8 N \varphi(N)+\sum_{\substack{q<2 \sqrt{2} N \\(N, q) \neq 1}} \varphi(q)
$$

Finalmente, debemos eliminar los elementos que se encuentran en ambos conjuntos, no obstante, el número en el segundo miembro de (3.54) es una cota superior para las ternas $(N, q, n) \in \mathbb{N}^{3}$ donde las propiedades P1 y P2 se cumplen. Definamos la aplicación $\mathcal{T}: \mathfrak{g}^{*} \rightarrow \mathbb{R}^{2}$ por

$$
\left(h_{3}, h_{4}\right) \mapsto\left(\frac{T\left(h_{3}\right)}{2 \pi}, \frac{T\left(h_{4}\right)}{2 \pi}\right) .
$$

Esta aplicación tiene como dominio $\operatorname{Dom}(\mathcal{T})=(-2,0) \times(-2,0)$ y su imagen es $\operatorname{Img}(\mathcal{T})=\left(\frac{1}{2 \sqrt{2}}, \infty\right) \times\left(\frac{1}{2 \sqrt{2}}, \infty\right)$.

$$
\mathbb{R}^{4} \supset M \stackrel{\mu}{\longrightarrow} \mathfrak{g}^{*} \stackrel{\mathcal{T}}{\longrightarrow} \mathbb{R}^{2}
$$

La terna $(N, q, n) \in \mathbb{N}^{3}$ induce un punto $y \in \mathcal{T}\left(\mathfrak{g}^{*}\right)$ con componentes $y=\left(\frac{N}{q}, \frac{N}{n}\right)$ tal que el toro Lagrangiano $\mathbb{T} \subset \mathbb{R}^{4}$ obtenido mediante

$$
\mathbb{T}=\left(\mu^{-1} \circ \mathcal{T}^{-1}\right)(y),
$$

está foliado por órbitas periódicas de periodo $2 N \pi$, Por lo tanto es un toro resonante $(\mathbb{T} \cap M) \subset \Sigma \in \mathfrak{M}$.

Demostración. [Teorema $\mathbf{3 . 2 3}$ La primera parte del teorema es una consecuencia del hecho que la unión numerable de conjuntos finitos es un conjunto numerable. Usando los Lemas 3.13 y 3.14 tenemos que el número de toros resonantes es numerable y debido a que cada toro pertenece exactamente a una superficie de energía, el conjunto $\mathfrak{M}$ también es numerable.

Ahora debemos probar que el conjunto de valores $h_{*}$ de superficies de energía que contienen toros resonantes es denso en $(-4,0)$, y tiene medida cero.

Para cada punto racional $y \in \operatorname{Img}(\mathcal{T})$ con $y=\left(\frac{r}{s}, \frac{u}{v}\right),(r, s)=1$ y $(u, v)=$ 1 , construimos el punto $\left(\frac{r u}{m}, \frac{s u}{m}, \frac{r v}{m}\right) \in \mathbb{N}^{3}$ donde $m=\operatorname{mcd}(r u, s u, r v)$. Como este punto cumple las propiedades P1 y P2 de la Definición 3.5. existe un toro resonante foliado por órbitas periódicas con periodo

$$
\tau=2 \frac{r u}{m} \pi=\frac{s u}{m} T\left(h_{3}\right)=\frac{r v}{m} T\left(h_{4}\right)
$$

El conjunto de valores racionales de $\mathcal{T}$ que llamaremos $\mathscr{Q}:=\operatorname{Img}(\mathcal{T}) \cap \mathbb{Q}^{2}$ es un subconjunto denso de medida cero en $\operatorname{Img}(\mathcal{T})$. La aplicación $\mathcal{T}$ es continua ya que cada componente es continua y entonces $\mathcal{T}^{-1}(\mathscr{Q}) \subset \mathfrak{g}^{*}$ es un subconjunto denso en la imagen de la aplicación momento $\mu$. Ahora construimos la función $\mathcal{H}: \mathfrak{g}^{*} \rightarrow \mathbb{R}$ tal que envía $x=\left(h_{3}, h_{4}\right) \mapsto h_{3}+$ $h_{4}$. Es inmediato que $\mathcal{H}\left(\mathcal{T}^{-1}(\mathscr{Q})\right) \subset(-4,0)$ es un subconjunto denso por continuidad, y tiene medida cero ya que $\mathscr{Q}$ es un conjunto numerable. 
3.6 SOluCiOnes PeRiódicAs En EL PROBlema CIRCUlar: $m_{3}=m_{4} . \quad 93$

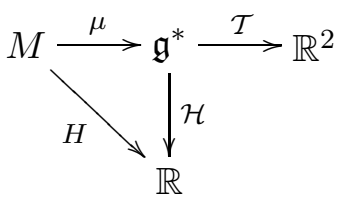

Nota 3.12. Debemos recordar en todos los cálculos que debido a la singularidad y a la condición $q_{3}>q_{4}$, todas las fibras $\mu^{-1}(x)$ deben restringirse a la variedad abierta $M$. 



\section{CAPÍTULO 4}

\section{CONTINUACión DE ÓRBiTAS PERIÓDICAS}

Como hemos visto en el capítulo anterior, las soluciones del problema se mantienen en un mismo toro invariante si y sólo si la condición $\epsilon\left(p_{3}+p_{4}\right)=0$ se cumple. En caso contrario, se tendrá un intercambio de energía entre los secundarios y la solución deberá continuarse en un toro diferente. Esto provoca una gran sensibilidad a producir órbitas aleatorias bajo pequeñas perturbaciones de las condiciones iniciales.

En este capítulo, estudiaremos la forma de continuar órbitas periódicas a través de colisiones que se preserven sobre el mismo toro invariante para el caso en que las masas de los secundarios son diferentes. La forma de hacerlo es considerar que el parámetro $\epsilon$ pasa de cero a un valor positivo y pequeño.

\subsection{CONTINUACIÓN DE ÓRBitas SIMÉTRICAS}

4.1.1. Condiciones Uno de los métodos para encontrar órbitas periódicas es considerar las soluciones que pasan por la sección cero en dos puntos distintos. En el argot de mecánica celeste se dice que son soluciones que "tocan" al conjunto de velocidad cero en dos puntos distintos. En particular, las órbitas simétricas del problema de Sitnikov con $2+2$ cuerpos pasan por la sección cero y esto se cumple aún en el caso elíptico. Sin embargo, sólo en el caso circular se obtienen órbitas cerradas para cualquier valor de $h \in(-4,0)$.

Las órbitas simétricas en el problema circular de Sitnikov de $2+2$ cuerpos, sólo existen para el caso $\epsilon=0$, es decir, cuando las masas infinitesimales de los secundarios son iguales. Estas órbitas se encuentran sobre las fibras 
$\mathscr{T}_{x}=\mu^{-1}(x)$ de los elementos que pertenecen a la diagonal de la imagen de la aplicación momento

$$
\mathscr{D}=\left\{x \in \mu(M) \subset \mathfrak{g}^{*} \mid x=\left(h_{3}, h_{3}\right)\right\},
$$

formalicemos estas afirmaciones.

LEMA 4.15 (Jiménez-Pérez). El problema de Sitnikov de 2+2 cuerpos tiene órbitas simétricas no triviales si y sólo si las masas infinitesimales de los secundarios son iguales.

Demostración. Supongamos primero que $m_{3}=m_{4}$, entonces para las condiciones iniciales $q_{3}(0)=-q_{4}(0)$ y $p_{3}(0)=-p_{4}(0)$ se tendrá que $q_{3}(t)=-q_{4}(t)$ y $p_{3}(t)=-p_{4}(t)$ para toda $t \in I \subset \mathbb{R}$ ya que los subsistemas $H_{3}\left(q_{3}, p_{3}\right)$ y $H_{4}\left(q_{4}, p_{4}\right)$ son idénticos.

Por el otro lado, suponiendo que se tiene una órbita simétrica entonces tenemos que $q_{3}(t)=-q_{4}(t)$ y $p_{3}(t)=-p_{4}(t)$ para toda $t \in I \subset \mathbb{R}$, por ello $\dot{q}_{3}(t)=-\dot{q}_{4}(t)$. Por la definición de los momentos (2.13) se debe satisfacer simultáneamente que

$$
\dot{q}_{3}(t)=-\frac{(1-\epsilon)}{(1+\epsilon)} \dot{q}_{4}(t), \quad \mathrm{y} \quad \dot{q}_{3}(t)=-\dot{q}_{4}(t) .
$$

Como la órbita periódica no debe ser trivial debemos excluir el punto fijo $q_{3}(t) \equiv q_{4}(t)=0$. Por lo tanto (4.2) se satisface si y sólo si $\epsilon=0$. Es decir, $m_{3}=m_{4}$.

Es importante ver que el resultado anterior se aplica tanto al caso circular como al elíptico. En el caso elíptico no existe certeza que las condiciones iniciales simétricas elegidas generen una órbita periódica. El siguiente resultado se relaciona con el caso circular en donde podemos utilizar la aplicación momento definida en (3.22).

Lema 4.16 (Jiménez-Pérez). En el problema circular de Sitnikov con $2+2$ cuerpos con aplicación momento $\mu: M \mapsto \mathfrak{g}^{*}$ definida en (3.22), la fibra $\mathscr{T}_{x}=\mu^{-1}(x)$ tiene una órbita periódica simétrica (no trivial) si y sólo si $x \in \mathscr{D}$ y $\epsilon=0$.

Demostración. Por el Lema 4.15 sabemos que las órbitas simétricas implican $\epsilon=0$. La expresión para la aplicación momento será simétrica en las coordenadas $\left(q_{3}, p_{3}\right)$ y $\left(q_{4}, p_{4}\right)$

$$
\mu\left(q_{3}, p_{3}, q_{4}, p_{4}\right)=\left(\frac{1}{2} p_{3}^{2}-\frac{1}{\sqrt{q_{3}^{2}+1 / 4}}, \frac{1}{2} p_{4}^{2}-\frac{1}{\sqrt{q_{4}^{2}+1 / 4}}\right) .
$$

Utilizando que las órbitas simétricas deben cumplir $q_{3}(t)=-q_{4}(t)$ y $p_{3}(t)=$ $-p_{4}(t)$ para toda $t \in I \subset \mathbb{R}$, tenemos

$$
\begin{aligned}
\mu\left(q_{3}, p_{3},-q_{3},-p_{3}\right) & =\left(\frac{1}{2} p_{3}^{2}-\frac{1}{\sqrt{q_{3}^{2}+1 / 4}}, \frac{1}{2} p_{3}^{2}-\frac{1}{\sqrt{q_{3}^{2}+1 / 4}}\right) \\
& =\left(h_{3}, h_{3}\right)=x .
\end{aligned}
$$


Esto indica que $x \in \mathscr{D}$.

Para demostrar la inversa sólo debemos ver que dado $\epsilon=0$ entonces la fibra $\mathscr{T}_{x}$ corresponde a un toro con generadores idénticos. Sea $\varphi(t)=$ $(q(t), p(t))$ una solución del problema circular clásico de Sitnikov con energía $h_{3}$. Las soluciones sobre $\mathscr{T}_{x}$ tienen la forma $\sigma(t)=\left(\varphi(t), \varphi\left(t+t_{1}\right)\right)$ para $t_{1} \in(0, T)$ donde $T=T\left(h_{3}\right)$ es el periodo de la solución $\varphi(t)$ con energía $h_{3}$. Si $t_{1}=T / 2$ entonces $\varphi(t+T / 2)=-\varphi(t)$ y designando $\left(q_{3}(t), p_{3}(t)\right)=\varphi(t)$ y $\left(q_{4}(t), p_{4}(t)\right)=\varphi(t+T / 2)$ se tendrá

$$
\begin{aligned}
\sigma\left(t_{0}\right) & =\left(q_{3}\left(t_{0}\right), p_{3}\left(t_{0}\right), q_{4}\left(t_{0}\right), p_{4}\left(t_{0}\right)\right) \\
& =\left(q_{3}\left(t_{0}\right), p_{3}\left(t_{0}\right),-q_{3}\left(t_{0}\right),-p_{3}\left(t_{0}\right)\right),
\end{aligned}
$$

para toda $t_{0} \in I \subset \mathbb{R}$. La solución anterior corresponde a una órbita simétrica sobre $\mathscr{T}_{x}$. La órbita es periódica porque estamos en el problema circular.

Para hacer la continuación de soluciones, debemos considerar las características que deseamos preservar de las soluciones simétricas anteriormente descritas. Supongamos que $\epsilon \neq 0$, entonces las ecuaciones de movimiento y la función Hamiltoniana ya no tienen la simetría

$$
\left(q_{3}, p_{3}, q_{4}, p_{4}\right) \mapsto\left(q_{4}, p_{4}, q_{3}, p_{3}\right) .
$$

La forma de proceder será considerar que la solución debe pasar por la sección cero para un tiempo $\tau_{0}=T / 2$ que consideraremos la mitad del periodo

$$
\sigma\left(\tau_{0}\right)=\left(q_{3}\left(\tau_{0}\right), q_{4}\left(\tau_{0}\right), 0,0\right) .
$$

Además, debemos buscar que al momento de la colisión se preserve la energía de cada secundario. Por el Lema 3.12 sabemos que se preserva la energía si y sólo si $p_{3}=-p_{4}$ en el momento de la colisión por lo que se tendrá que cumplir

$$
\sigma(0)=\left(q_{0}, q_{0}, p_{0},-p_{0}\right),
$$

o sea $\sigma(0) \in \mathcal{P}$ y $\sigma(T / 2) \in \mathcal{L}_{0}$ donde $\mathcal{L}_{0}$ es la sección cero y es un plano Lagrangiano que corresponde a la inclusión canónica del espacio de configuración $\mathcal{Q} \hookrightarrow M$. La sección cero contiene los conjuntos de velocidad cero de un sistema mecánico para todos los valores fijos de la energía $H=h$.

Para simplificar el análisis consideraremos una solución como el producto cartesiano de dos soluciones del problema de Sitnikov independientes y graficaremos las posiciones con respecto del nuevo tiempo uniformizante $\tau$. Es decir, para la solución $\sigma(\tau)=\left(q_{3}(\tau), q_{4}(\tau), p_{3}(\tau), p_{4}(\tau)\right)$ graficaremos en un mismo marco de referencia $(\tau, q)$ las soluciones $\left(\tau, q_{3}(\tau)\right)$ y $\left(\tau, q_{4}(\tau)\right)$ con la condición $q_{3}(\tau) \geq q_{4}(\tau)$ para toda $\tau \in \mathbb{R}$. En la Figura 4.1 se pueden apreciar las dos soluciones independientes del problema de Sitnikov dibujadas de esta manera. Las imágenes son ilustrativas y no preservan la escala real. 


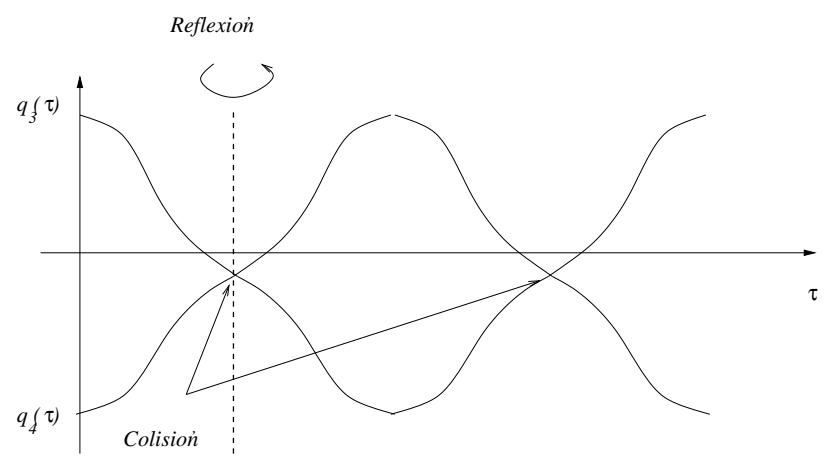

Figura 4.1. Continuación por reflexión y rebote elástico.

4.1.2. Cadenas de soluciones a través de COlisiones. En esta sección se considerarán las definiciones básicas para estudiar las órbitas de soluciones periódicas y cuasi-periódicas a través de colisiones. Para este fin, se utiliza el concepto de cadena donde se consideran como eslabones a los intervalos de soluciones que van de expulsión a colisión.

Definición 4.1. Decimos que un sistema Hamiltoniano para el problema de $N$-cuerpos tiene una trayectoria de colisión si existe una solución $\sigma(t)=$ $(q(t), p(t))$ contenida en $M$, tal que la componente $q:\left(t_{0}, t_{1}\right) \rightarrow \mathcal{Q}$, tiene al menos uno de los extremos en el conjunto $\Delta$. Es decir, $q\left(t_{i}\right) \in \Delta$, para $i=0,1$ y $q\left(t_{*}\right) \subset \mathcal{Q}$ para toda $t_{*} \in\left(t_{0}, t_{1}\right)$.

En general se puede dar que $t_{0}=-\infty$ o $t_{1}=\infty$ pero no se pueden dar ambos a la vez. Para el primer caso tendremos entonces una trayectoria de captura por colisión y en el segundo caso tendremos una trayectoria de escape a partir de colisión

Definición 4.2 (Bolotin-MacKay 12). Sea $a_{i} \in \mathcal{A}$ un conjunto ordenado numerable, una secuencia de trayectorias de colisión $\left(q_{a_{i}}\right)_{a_{i} \in \mathcal{A}}$ se llamará una cadena si se cumple simultáneamente que

- $q_{a_{i}}\left(\left(t_{1}\right)_{a_{i}}\right)=q_{a_{i+1}}\left(\left(t_{0}\right)_{a_{i+1}}\right) y$

- $p_{a_{i}}\left(\left(t_{1}\right)_{a_{i}}\right) \neq p_{a_{i+1}}\left(\left(t_{0}\right)_{a_{i+1}}\right)$,

para toda $a_{i} \in \mathcal{A}$.

Note que la segunda condición indica que la solución pasa por una singularidad. Es decir que en cada extremo, los segmentos son continuos pero no diferenciables en el espacio de configuración $\mathcal{Q}$.

De manera inmediata, deducimos que existe una discontinuidad en los fibrados tangente $T \mathcal{Q}$ y cotangente $T^{*} \mathcal{Q}$.

Definición 4.3. Definimos una cadena periódica de periodo $t_{n}$ si dada una cadena definida por $\left(a_{i}\right)_{i \in \mathbb{Z}}$ con $a_{i} \in \mathcal{A}$, existe $n \in \mathbb{N}$ tal que

- $q_{a_{i+n}}\left(\left(t_{1}\right)_{a_{i+n}}\right)=q_{a_{i}}\left(\left(t_{0}\right)_{a_{i}}\right) y$

- $q_{a_{i+m}}\left(\left(t_{1}\right)_{a_{i+m}}\right) \neq q_{a_{i}}\left(\left(t_{0}\right)_{a_{i}}\right)$ para $1 \leq m<n$. 
El periodo está dado por $t_{n}=\left(\left(t_{1}\right)_{a_{i+n}}-\left(t_{0}\right)_{a_{i}}\right)$.

Definición 4.4. Se define el orden de una cadena, como el número de elementos \# $\left(q_{a_{i}}\right)$ de la secuencia que la define.

En la definición de cadena periódica, el número $n$ es precisamente el orden y diremos que es una cadena periódica de orden $n$ o una $n$-cadena periódica.

Es importante ver que una solución del problema circular restringido de Sitnikov con $2+2$ cuerpos a través de colisiones se puede estudiar mediante la dinámica de los puntos sobre la variedad de colisión.

Si consideramos el problema restringido de $2+2$ cuerpos de tal forma que las masas de los secundarios tienden a cero con una relación $m_{4}=\frac{1-\epsilon}{1+\epsilon} m_{3}$ y si el valor del parámetro es $\epsilon \neq 0$, entonces toda solución que pasa a través de una colisión se mantiene en el mismo toro invariante si y sólo si $p_{3}+p_{4}=0$. En este caso es fácil encontrar soluciones periódicas como una extensión de las órbitas periódicas del caso $\epsilon=0$, cuando los tiempos de retorno de los cuerpos secundarios son iguales $T\left(h_{3}\right)=T\left(h_{4}\right)$ con $-2<h_{3}, h_{4}<0$.

Otro caso interesante para estudiar son las órbitas periódicas para cadenas de orden $n>1$ con intercambio de energía. Este tipo de soluciones producen un "salto" de la solución entre distintos toros y se tiene cuando al momento de la colisión la velocidad del centro de masa relativo de los secundarios es diferente de cero $\frac{1+\epsilon}{2} \dot{q}_{3}+\frac{1-\epsilon}{2} \dot{q}_{4} \neq 0$ [1]. Como consecuencia inmediata se tiene que $p_{3}+p_{4} \neq 0$. Una solución de este tipo para una cadena de orden 2 donde la relación entre los tiempos de retorno de cada secundario es $2: 1$ se puede ver en la Figura 4.2 b. Nótese que al momento de la colisión se ha perdido la simetría entre las soluciones antes y después de la colisión.

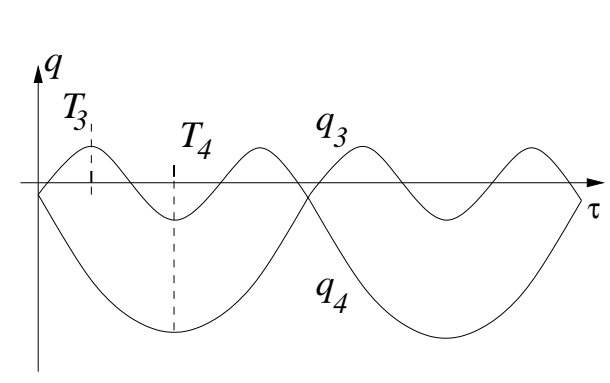

(a)

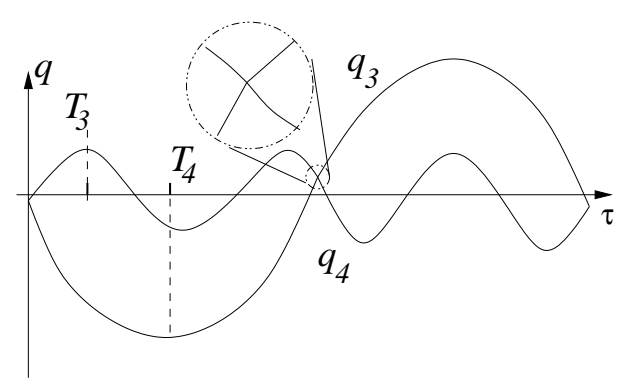

(b)

Figura 4.2. Dos órbitas periódicas para la relación $2: 1$.

En lo que resta de este capítulo estaremos interesados en estudiar la extensión de las órbitas periódicas para cadenas de orden 1 tal que los tiempos de retorno sean iguales $T\left(h_{3}\right)=T\left(h_{4}\right)$. 


\subsection{Cadenas DE ORDEN 1 EN EL PROBLEMA $2+2$}

En esta sección determinaremos la existencia de soluciones que pasan por los planos $\mathcal{P}$ y $\mathcal{L}_{0}$ de dimensión 2 en $\bar{M}:=M \cup \Delta$, en el sistema con función Hamiltoniana

$$
H=\left(\frac{1}{2(1+\epsilon)} p_{3}^{2}-\frac{1+\epsilon}{\sqrt{q_{3}^{2}+1 / 4}}\right)+\left(\frac{1}{2(1-\epsilon)} p_{4}^{2}-\frac{1-\epsilon}{\sqrt{q_{4}^{2}+1 / 4}}\right)
$$

asociado al problema circular de Sitnikov de $2+2$ cuerpos. Una vez que se tiene la existencia de dichas soluciones, la construcción de la órbita periódica es relativamente fácil. Iniciemos con algunos lemas que caracterizan las condiciones iniciales que se tomarán sobre el conjunto de colisiones y la sección cero.

Lema 4.17 (Jiménez-Pérez). Para toda $h \in(-4,0)$ fija y $\epsilon \in\left[0, \epsilon_{0}\right)$ arbitraria pero pequeña, el momento inicial $p_{0}=p_{3}(0)=-p_{4}(0)$ asociado a la solución con condiciones iniciales $\sigma_{0} \in \mathcal{P}$, es una función localmente suave de la posición $q_{0}$ y de los parámetros $h$ y $\epsilon$ fijos, en una vecindad de $\left(q_{0}, \epsilon\right)=(0,0)$.

Demostración. Sean $h$ y $\epsilon$ fijas en su dominio de definición. Considerando la solución con condiciones iniciales $\sigma_{0}=\sigma(0) \in \mathcal{P}$, se debe cumplir que

$$
p_{0}:=p_{3}(0)=-p_{4}(0) \quad \text { y } \quad q_{0}:=q_{3}(0)=q_{4}(0) .
$$

Substituyendo estos valores en la relación de energía, para $H=h$ fija se tiene

$$
h=\frac{1}{\left(1-\epsilon^{2}\right)} p_{0}^{2}-\frac{2}{\sqrt{q_{0}^{2}+1 / 4}} .
$$

Resolviendo para $p_{0}=p_{0}\left(q_{0} ; h, \epsilon\right)$ obtenemos

$$
p_{0}\left(q_{0} ; h, \epsilon\right)= \pm \sqrt{2\left(1-\epsilon^{2}\right)\left(\frac{h}{2}+\frac{1}{\sqrt{q_{0}^{2}+1 / 4}}\right)} .
$$

Esta es una función suave para valores de $q_{0}$ en el intervalo

$$
q_{0} \in\left(-\sqrt{\frac{4}{h^{2}}-\frac{1}{4}}, \sqrt{\frac{4}{h^{2}}-\frac{1}{4}}\right) .
$$

La elección del signo para $p_{0}\left(q_{0} ; h, \epsilon\right)$ se realiza analizando la solución de manera local.

Por lo tanto, para toda $q_{0}$ adecuada, se tienen condiciones iniciales sobre el plano $\mathcal{P}$ dadas por $\sigma_{0}=\left(q_{0}, q_{0}, p_{0},-p_{0}\right)$. Estas son las condiciones iniciales para una solución que sale de manera perpendicular de la recta $q_{3}=q_{4}$ en la proyección sobre el plano $\mathcal{L}_{0}$ (véase la Figura 4.3.) 


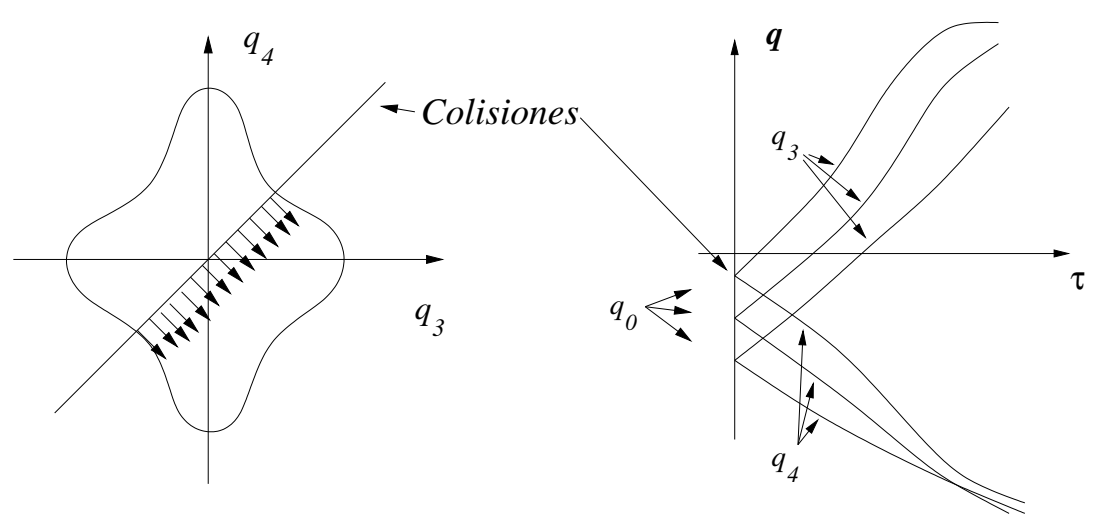

Figura 4.3. Soluciones con condiciones iniciales $\sigma(0) \in \mathcal{P}$.

Lema 4.18 (Jiménez-Pérez). Sea $x \in \mathfrak{g}^{*}$ con $x=\left(h_{3}, h_{4}\right)$ y consideremos una solución $\sigma(t)$ sobre la fibra $\mathscr{T}_{x}$, tal que $\sigma(0) \in \mathcal{P}$. Entonces, los niveles de energía relativa $h_{3}$ y $h_{4}$ cumplen

$$
h_{3}=\frac{h}{2}-\epsilon\left(\frac{h}{2}+\frac{2}{\sqrt{q_{0}^{2}+\frac{1}{4}}}\right) \quad \text { y } \quad h_{4}=\frac{h}{2}+\epsilon\left(\frac{h}{2}+\frac{2}{\sqrt{q_{0}^{2}+\frac{1}{4}}}\right),
$$

donde $h=h_{3}+h_{4}$ es la energía del sistema.

Demostración. Sea $H=h$ fija y consideremos $h=h_{3}+h_{4}$

$$
h=\underbrace{\left(\frac{1}{2(1+\epsilon)} p_{3}^{2}-\frac{1+\epsilon}{\sqrt{q_{3}^{2}+1 / 4}}\right)}_{h_{3}}+\underbrace{\left(\frac{1}{2(1-\epsilon)} p_{4}^{2}-\frac{1-\epsilon}{\sqrt{q_{4}^{2}+1 / 4}}\right)}_{h_{4}}
$$

Como la solución particular cumple con las condiciones iniciales $\sigma_{0} \in \mathcal{P}$, con $q_{0}=q_{3}(0)=q_{4}(0)$ y $p_{0}=p_{3}(0)=-p_{4}(0)$ entonces se tiene

$$
\begin{aligned}
& h_{3}=\frac{1}{2(1+\epsilon)} p_{0}^{2}-\frac{1+\epsilon}{\sqrt{q_{0}^{2}+1 / 4}} \\
& h_{4}=\frac{1}{2(1-\epsilon)} p_{0}^{2}-\frac{1-\epsilon}{\sqrt{q_{0}^{2}+1 / 4}} .
\end{aligned}
$$

Utilizando la expresión (4.8) para $p_{0}$ se obtiene el resultado deseado.

Ahora debemos considerar las condiciones para que la solución pase por el plano $\mathcal{L}_{0}$, es decir, las soluciones tales que para $T\left(h_{0}\right) / 4=\tau_{0}$ se tenga $\sigma\left(\tau_{0}\right)=\left(q_{3}, q_{4}, 0,0\right)$. Estas soluciones se conocen como las soluciones que "tocan" el conjunto de velocidad cero, que en este caso es una curva.

A partir de este momento, solo estaremos interesados en este tipo de soluciones, en una vecindad alrededor del hiperplano $q_{3}=-q_{4}$, es decir, de la solución con relación de periodos de los secundarios $1: 1$. Esta curva debe intersectar a la curva de velocidad cero $\mathcal{Z}$ (véase la Figura 4.4.). 


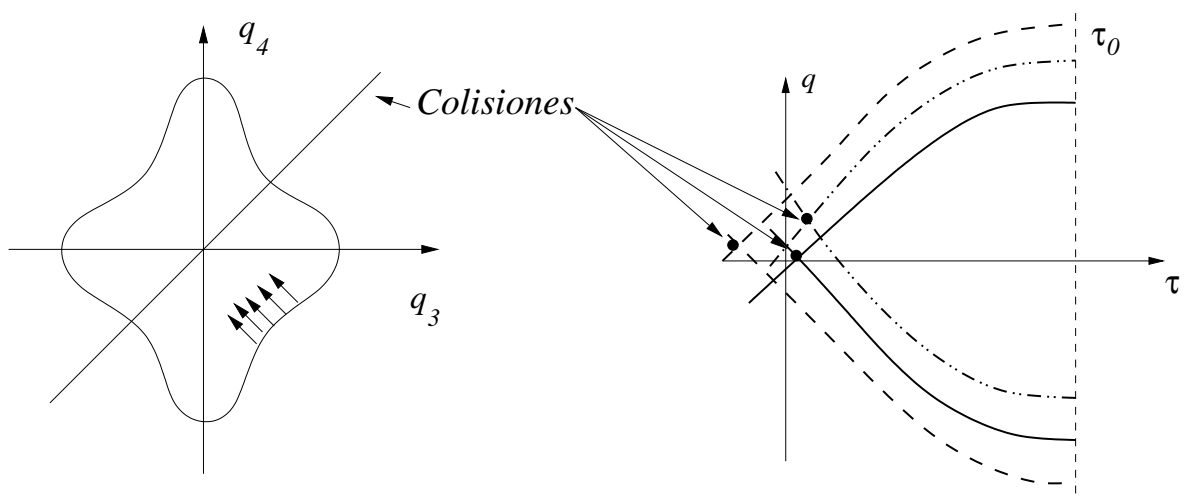

FigurA 4.4. Soluciones con condiciones $\sigma\left(\tau_{0}\right) \in \mathcal{L}_{0}$.

Dado que ambas condiciones dependen de manera continua de $\epsilon$, ahora buscaremos una solución $\hat{\sigma}$ que pase por ambos planos $\mathcal{L}_{0}$ y $\mathcal{P}$ y se verá de manera parecida al de la Figura 4.5
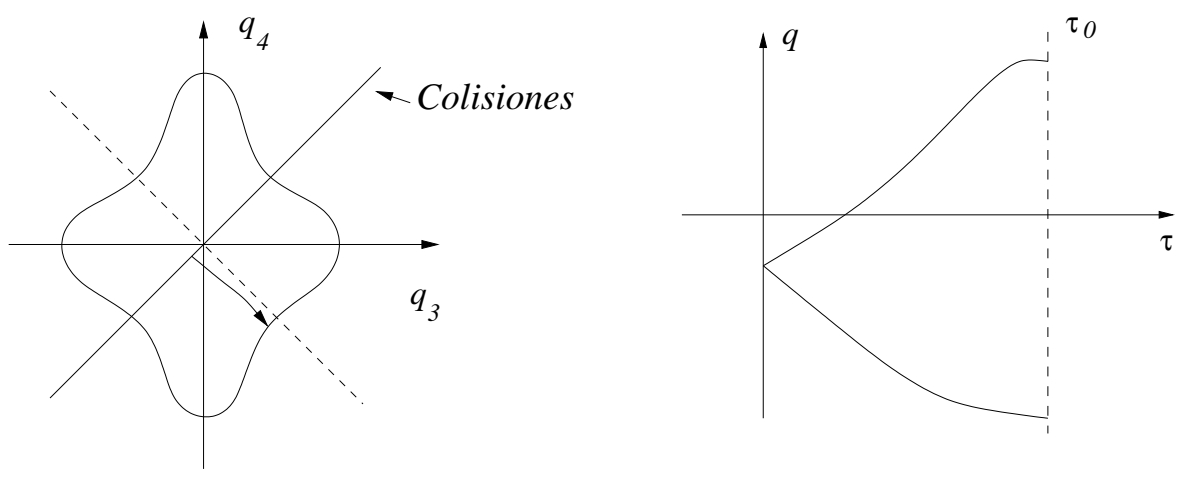

Figura 4.5. Solución buscada.

Proposición 4.23 (Jiménez-Pérez). Para todo valor $0 \leq \epsilon<\epsilon_{0}$ fijo y pequeño y para toda $h \in(-4,0)$, existe un valor $q_{0} \in\left(-\sqrt{\frac{4}{h^{2}}-\frac{1}{4}}, 0\right)$, tal que la solución con condiciones iniciales $\sigma(0) \in \mathcal{P}$ pasa por el plano $\mathcal{L}_{0}$.

Demostración. La demostración de este resultado lo haremos utilizando las propiedades de continuidad de las soluciones.

Consideremos $q_{0}=0$. Evaluando en las expresiones (4.10) de las energías relativas obtenemos

$$
h_{3}=\frac{h}{2}-\epsilon\left(\frac{h}{2}+4\right) \quad h_{4}=\frac{h}{2}+\epsilon\left(\frac{h}{2}+4\right)
$$

Además, $h \in(-4,0)$ de donde $2<\left(\frac{h}{2}+4\right)<4$ y por lo tanto tenemos la relación $h_{3}<h / 2<h_{4}$. 
El inciso 3 del Teorema 3.22 nos asegura que la función periodo para las soluciones del problema de Sitnikov es una función creciente. Por lo tanto $T\left(h_{3}\right)<T(h / 2)<T\left(h_{4}\right)$. Por hipótesis $q_{3}>q_{4}$ y considerando que $p_{i}\left(\tau ; \sigma_{0}\right)=m_{i} \dot{q}_{i}\left(\tau, \sigma_{0}\right)$ es el momento asociado a la solución con condiciones iniciales $\sigma_{0}$ al tiempo $\tau$, entonces

$$
p_{3}\left(\tau ; \sigma_{0}\right)\left\{\begin{array}{lll}
>0 & \text { si } & 0 \leq \tau<\frac{T\left(h_{3}\right)}{4}, \\
=0 & \text { si } \quad \tau=\frac{T\left(h_{3}\right)}{4}, \\
<0 & \text { si } \quad \frac{T\left(h_{3}\right)}{4}<\tau<\frac{3 T\left(h_{3}\right)}{4},
\end{array}\right.
$$

mientras que

$$
p_{4}\left(\tau ; \sigma_{0}\right)<0 \quad \text { si } \quad 0 \leq \tau<\frac{T\left(h_{4}\right)}{4} .
$$

Como $T\left(h_{3}\right)<T\left(h_{4}\right)$ entonces

$$
\left.\begin{array}{l}
p_{3}\left(\tau ; \sigma_{0}\right)=0 \\
p_{4}\left(\tau ; \sigma_{0}\right)<0
\end{array}\right\} \quad \text { si } \quad \tau=\frac{T\left(h_{3}\right)}{4} .
$$

Recordemos que $p_{0}\left(q_{0} ; h, \epsilon\right)$ es una función suave de $q_{0}$ y de los parámetros así como del tiempo. Consideremos $\tau_{3}$ el tiempo que tarda en llegar la variable $p_{3}(\tau)$ de la condición inicial $p_{3}(0)=p_{3}\left(0, q_{0} ; h, \epsilon\right)$ al valor $p_{3}\left(\tau_{3}\right)=0$ y definimos de la misma forma el tiempo $\tau_{4}$ para la variable $p_{4}(\tau)$. Si $\epsilon=0$ y $q_{0}=0$ entonces $\tau_{3}=\tau_{4}=T(h / 2) / 4$ mientras que si $\epsilon>0$ con $q_{0}=0$ entonces $\tau_{3}=T\left(h_{3}\right) / 4$ y $\tau_{4}=T\left(h_{4}\right) / 4$ con $\tau_{3}<\tau_{4}$ (véase la Figura 4.6 izquierda).

Disminuyamos la variable independiente $q_{0}$ de manera continua, entonces el valor de $\tau_{3}$ se incrementa mientras que el valor de $\tau_{4}$ disminuye.

Consideremos el intervalo entre los cuartos de periodo

$$
\Delta_{\tau}=\frac{1}{4}\left(T\left(h_{4}\right)-T\left(h_{3}\right)\right)
$$

y evaluemos la solución del problema de Sitnikov para los valores de la energía $h_{3}$ y $h_{4}$ en el tiempo $\tau=\Delta_{\tau}$. A estos valores los denotaremos por $\hat{q}_{3}$ y $\hat{q}_{4}$.
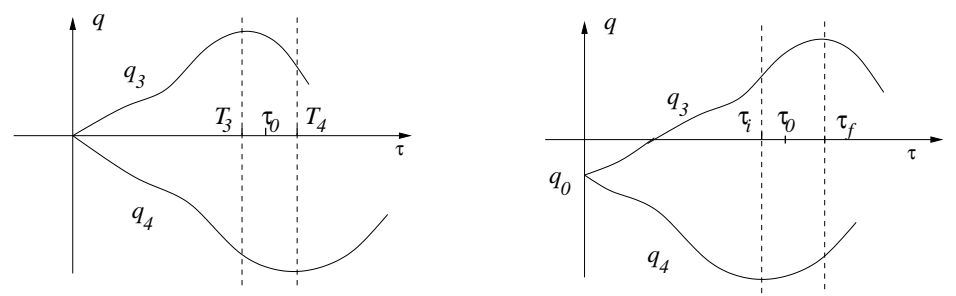

FigurA 4.6. Soluciones para $q_{0}=0$ y $q_{0}=-\operatorname{máx}\left\{\hat{q}_{3}, \hat{q}_{4}\right\}$.

La solución con condiciones iniciales $q_{0}=-\operatorname{máx}\left\{\hat{q}_{3}, \hat{q}_{4}\right\}$ y $p_{3}(0)=$ $p_{3}\left(0, q_{0} ; h, \epsilon\right)$ cumple que $\tau_{3} \geq \frac{1}{4} T\left(h_{3}\right)+\Delta_{\tau}$ y $\tau_{4} \leq \frac{1}{4} T\left(h_{4}\right)-\Delta_{\tau}$, es decir $\tau_{3} \geq \frac{1}{4} T\left(h_{4}\right)$ y $\tau_{4} \leq \frac{1}{4} T\left(h_{3}\right)$. Esto indica que para la condición inicial 
$q_{0}=-\operatorname{máx}\left\{\hat{q}_{3}, \hat{q}_{4}\right\}$, se tiene

$$
\left.\begin{array}{l}
p_{3}\left(\tau ; \sigma_{0}\right)=0 \\
p_{4}\left(\tau ; \sigma_{0}\right)>0
\end{array}\right\} \quad \text { si } \tau=\tau_{3} .
$$

Por continuidad existe $q_{0} \in\left(-\operatorname{máx}\left\{\hat{q}_{3}, \hat{q}_{4}\right\}, 0\right)$ tal que a un tiempo $\tau_{3}$ se tiene $p_{4}\left(\tau_{3} ; \sigma_{0}\right)=0$. y por lo tanto, la solución cumple $p_{3}\left(\tau_{3}\right)=p_{4}\left(\tau_{3}\right)=0$ es decir que cruza el plano $\mathcal{L}_{0}$.

Como corolario se tiene una aplicación directa al problema circular de Sitnikov de $2+2$ cuerpos.

Corolario 3 (Jiménez-Pérez). Para toda $h \in(-4,0)$ fija y para toda $\epsilon \in\left(0, \epsilon_{0}\right)$ arbitraria y pequeña, el sistema Hamiltoniano $\left(M, \omega, X_{H}\right)$ definido por la función (4.5) tiene al menos una solución $\hat{\sigma}(\tau)$ tal que $\sigma(0) \in \mathcal{P}$ y $\sigma\left(\tau_{\text {col }}\right) \in \mathcal{L}_{1}$ para algún $\tau_{\text {col }}>0$.

Demostración. La demostración de la Proposición 4.23 muestra una solución a través de una cadena de orden 1 y cuya relación entre $T\left(h_{3}\right)$ y $T\left(h_{4}\right)$ de tipo 1:1.

LEMA 4.19 (Jiménez-Pérez). Supóngase que se tiene el valor de $q_{0} \in$ $\left(-\operatorname{máx}\left\{\hat{q}_{3}, \hat{q}_{4}\right\}, 0\right)$ tal que la solución $\sigma(\tau)$ con condiciones iniciales $\sigma_{0} \in \mathcal{P}$ cruza el plano $\mathcal{L}_{0}$. Entonces el tiempo que toma la solución para ir de $\mathcal{P}$ a $\mathcal{L}_{0}$ se encuentra en el intervalo $\tau_{0} \in\left(\frac{T\left(h_{3}\right)}{4}, \frac{T\left(h_{4}\right)}{4}\right)$.

Demostración. Esto es inmediato del hecho que para $\epsilon>0$ se tiene la relación entre las energías relativas $h_{3}<h_{4}$ y por lo tanto $T\left(h_{3}\right)<T\left(h_{4}\right)$. Consideremos la solución $\hat{\sigma}$ que cumple $\hat{\sigma}(0) \in \mathcal{L}_{0}$ y para alguna $\tau>0$ cumple $\hat{\sigma}(\tau) \in \mathcal{P}$.

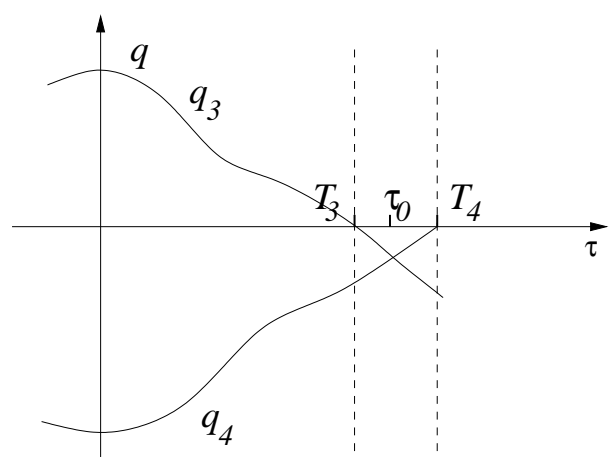

FiguRA 4.7. Estimación para $\tau_{0}$.

Se tiene $q_{3}\left(T\left(h_{3}\right) / 4\right)=0$ y $q_{4}\left(T\left(h_{4}\right) / 4\right)=0$; si consideramos la función $Q=q_{3}-q_{4}$ en los extremos del intervalo obtenemos $Q\left(T\left(h_{3}\right) / 4\right)>0 \mathrm{y}$ $Q\left(T\left(h_{4}\right) / 4\right)<0$. Por lo tanto, existe $\tau_{0} \in\left(\frac{T\left(h_{3}\right)}{4}, \frac{T\left(h_{4}\right)}{4}\right)$ tal que $Q\left(\tau_{0}\right)=0$.

Dicha $\tau_{0}$ es el valor buscado ya que $\hat{\sigma}\left(\tau_{0}\right) \in \mathcal{P}$ (véase la Figura 4.7) 
Lema 4.20 (Jiménez-Pérez). El valor $\tau_{0}$ del Lema 4.19 cumple la condición

$$
\left.\frac{\partial \tau_{0}}{\partial \epsilon}\right|_{\epsilon=0} \neq 0
$$

Demostración. Consideremos las soluciones sobre un toro invariante $\mathscr{T}_{x}=\mu^{-1}(x)$ para $x \in U \subset \mathfrak{g}^{*}$ donde $U=(-2,0) \times(-2,0)$. Como $x$ tiene la forma $x=\left(h_{3}, h_{4}\right)$, entonces los momentos asociados $p_{3}$ y $p_{4}$ cumplen

$$
\begin{aligned}
& p_{3}^{2}=2(1+\epsilon)\left(h_{3}+\frac{1}{\sqrt{q_{3}^{2}+1 / 4}}\right), \\
& p_{4}^{2}=2(1-\epsilon)\left(h_{4}+\frac{1}{\sqrt{q_{4}^{2}+1 / 4}}\right) .
\end{aligned}
$$

Ahora sustituyamos las expresiones (4.10) del Lema 4.18 para obtener

$$
\begin{aligned}
& p_{3}^{2}=2(1+\epsilon)\left(\frac{h}{2}-\epsilon\left(\frac{h}{2}+\frac{2}{\sqrt{q_{0}^{2}+1 / 4}}\right)+\frac{1}{\sqrt{q_{3}^{2}+1 / 4}}\right) \\
& p_{4}^{2}=2(1-\epsilon)\left(\frac{h}{2}+\epsilon\left(\frac{h}{2}+\frac{2}{\sqrt{q_{0}^{2}+1 / 4}}\right)+\frac{1}{\sqrt{q_{4}^{2}+1 / 4}}\right)
\end{aligned}
$$

De cualquiera de ambas expresiones se obtiene el tiempo que utiliza la solución para ir del plano $\mathcal{P}$ al plano $\mathcal{L}_{1}$; este intervalo de tiempo es el valor de $\tau_{0}$ del Lema 4.19. Utilicemos la primera relación que produce la ecuación

$$
\tau_{0}=\int_{q_{0}}^{q_{3 \max }} \frac{d q_{3}}{\sqrt{\frac{1-\epsilon}{1+\epsilon} h-\frac{4 \epsilon}{1+\epsilon} \frac{1}{\sqrt{q_{0}^{2}+1 / 4}}+\frac{2}{1+\epsilon} \frac{1}{\sqrt{q_{3}^{2}+1 / 4}}}} .
$$

Si $\epsilon>0$ entonces $q_{0}<0$. Además, la distancia máxima del cuerpo con posición $q_{3}$ se obtiene cuando $p_{3}=0$. Utilizando (4.12) con $p_{3}=0$ obtenemos

$$
q_{3 \text { máx }}=\sqrt{\frac{(1+\epsilon)^{2}}{\left(-\frac{h}{2}+\epsilon\left(\frac{h}{2} \frac{2}{\sqrt{q_{0}^{2}+1 / 4}}\right)\right)^{2}}-\frac{1}{4}},
$$

donde vemos que $q_{3 \max }=q_{3 \max }\left(q_{0}, h, \epsilon\right)$.

El valor de $\tau_{0}=\tau_{0}\left(q_{3 \max }, q_{0}, h, \epsilon\right)$ solo depende de $q_{0}$, de la energía y de $\epsilon$. Por hipótesis $q_{0}<0$ es un valor dado, entonces por la regla de la cadena tenemos

$$
\left.\partial_{\epsilon}\left(\tau_{0}\right)\right|_{\epsilon=0}=\left\{\left.\int_{q_{0}}^{q_{3 \max }} \partial_{\epsilon}\left(\frac{1}{\sqrt{g\left(q_{3} ; q_{0}, h, \epsilon\right)}}\right)\right|_{\epsilon=0} d q_{3}\right\} \times\left(\left.\partial_{\epsilon}\left(q_{3 \text { máx }}\right)\right|_{\epsilon=0}\right),
$$

donde $\partial_{\epsilon}=\frac{\partial}{\partial \epsilon} \mathrm{y}$

$$
g\left(q_{3} ; q_{0}, h, \epsilon\right)=\frac{2}{1+\epsilon}\left(\frac{h}{2}+\frac{1}{\sqrt{q_{3}^{2}+1 / 4}}-\epsilon\left(\frac{h}{2}+\frac{2}{\sqrt{q_{0}^{2}+1 / 4}}\right)\right) .
$$


Operando de manera independiente sobre los diferentes términos de la ecuación tenemos las derivadas parciales

$$
\partial_{\epsilon}\left(\frac{1}{\sqrt{g\left(q_{3} ; q_{0}, h, \epsilon\right)}}\right)=2 \frac{h+\frac{2}{\sqrt{q_{0}^{2}+1 / 4}}}{g\left(q_{3} ; q_{0}, h, \epsilon\right)^{\frac{3}{2}}}
$$

y

$$
\partial_{\epsilon}\left(q_{3 \text { máx }}\right)=-\frac{(1+\epsilon)\left(h+\frac{2}{\sqrt{q_{0}^{2}+1 / 4}}\right)}{\sqrt{\frac{(1+\epsilon)^{2}}{\left(-\frac{h}{2}+\epsilon\left(\frac{h}{2} \frac{2}{\sqrt{q_{0}^{2}+1 / 4}}\right)\right)^{2}}-\frac{1}{4}}\left(-\frac{h}{2}+\epsilon\left(\frac{h}{2} \frac{2}{\sqrt{q_{0}^{2}+1 / 4}}\right)\right)^{3}}
$$

Evaluando en $\epsilon=0$ las expresiones para $q_{3 \text { máx }}, g\left(q_{3} ; q_{0}, h, \epsilon\right)$ y las derivadas parciales tenemos

$$
\begin{gathered}
\left.q_{3 \text { máx }}\right|_{\epsilon=0}=\sqrt{\frac{4}{h^{2}}-\frac{1}{4}}, \\
g\left(q_{3} ; q_{0}, h, 0\right)=\left(h+\frac{2}{\sqrt{q_{3}^{2}+1 / 4}}\right), \\
\left.\partial_{\epsilon}\left(\frac{1}{\sqrt{g\left(q_{3} ; q_{0}, h, \epsilon\right)}}\right)\right|_{\epsilon=0}=2 \frac{h+\frac{2}{\sqrt{q_{0}^{2}+1 / 4}}}{\left(h+\frac{2}{\sqrt{q_{3}^{2}+1 / 4}}\right)^{\frac{3}{2}}},
\end{gathered}
$$

y

$$
\left.\partial_{\epsilon}\left(q_{3 \text { máx }}\right)\right|_{\epsilon=0}=-\frac{\left(h+\frac{2}{\sqrt{q_{0}^{2}+1 / 4}}\right)}{\sqrt{\frac{4}{h^{2}}-\frac{1}{4}}\left(-\frac{h}{2}\right)^{3}}=-\frac{16\left(h+\frac{2}{\sqrt{q_{0}^{2}+1 / 4}}\right)}{h^{2} \sqrt{16-h^{2}}} \text {. }
$$

Sustituyendo (4.15), (4.17) y (4.18) en $\left.\partial_{\epsilon}\left(\tau_{0}\right)\right|_{\epsilon=0}$ tenemos

$$
\begin{aligned}
\left.\partial_{\epsilon}\left(\tau_{0}\right)\right|_{\epsilon=0} & =-\frac{16\left(h+\frac{2}{\sqrt{q_{0}^{2}+1 / 4}}\right)}{h^{2} \sqrt{16-h^{2}}}\left\{\int_{q_{0}}^{\sqrt{\frac{4}{h^{2}}-\frac{1}{4}}} 2 \frac{h+\frac{2}{\sqrt{q_{0}^{2}+1 / 4}}}{\left(h+\frac{2}{\sqrt{q_{3}^{2}+1 / 4}}\right)^{\frac{3}{2}}} d q_{3}\right\}, \\
& =-\frac{32\left(h+\frac{2}{\sqrt{q_{0}^{2}+1 / 4}}\right)^{2}}{h^{2} \sqrt{16-h^{2}}}\left\{\int_{q_{0}}^{\sqrt{\frac{4}{h^{2}}-\frac{1}{4}}} \frac{d q_{3}}{\left(h+\frac{2}{\sqrt{q_{3}^{2}+1 / 4}}\right)^{\frac{3}{2}}}\right\},
\end{aligned}
$$

Consideremos $q_{0} \in(-\delta, 0)$ pequeña, como $q_{0}<0<\sqrt{\frac{4}{h^{2}}-\frac{1}{4}}$ el integrando toma valores distintos en los límites de integración, entonces la integral 
definida no puede ser cero. La única posibilidad para que $\left.\partial_{\epsilon}\left(\tau_{0}\right)\right|_{\epsilon=0}=0$ es que

$$
\left(h+\frac{2}{\sqrt{q_{0}^{2}+1 / 4}}\right)^{2}=0,
$$

de donde se obtiene que

$$
q_{0}=-\sqrt{\frac{4}{h^{2}}-\frac{1}{4}}
$$

Eso implica que la parcial sólo se anula cuando $q_{0}$ se encuentra en el ínfimo de su dominio. Pero alrededor de $\epsilon=0$, se tiene que $q_{0} \in(-\delta, 0)$ lo que demuestra el lema.

Se sabe que para colisiones en un subsistema binario uno de los principales invariantes es la velocidad del centro de masa [41, pp. 236]. Para nuestro caso, la continuación de las órbitas periódicas tiene la característica que en los planos $\mathcal{L}_{0}$ y $\mathcal{P}$, la velocidad del centro de masa relativo de los secundarios es nula.

Entonces, la velocidad del centro de masa en dichos planos alcanza sus puntos extremos y por la simetría del centro de masa tenemos que el máximo debe ser exactamente el negativo del mínimo.

Este hecho nos permite considerar el siguiente resultado.

Lema 4.21. El valor $q_{0}$ de la Proposición 4.23 cumple la ecuación implícita

$$
\begin{aligned}
-2 q_{0}=(1+\epsilon) & \sqrt{\frac{\left(4 q_{0}^{2}+1\right)(1+\epsilon)^{2}}{\left(4 \epsilon-(1-\epsilon) h \sqrt{q_{0}^{2}+1 / 4}\right)^{2}}-\frac{1}{4}} \\
& -(1-\epsilon) \sqrt{\frac{\left(4 q_{0}^{2}+1\right)(1-\epsilon)^{2}}{\left(4 \epsilon+(1+\epsilon) h \sqrt{q_{0}^{2}+1 / 4}\right)^{2}}-\frac{1}{4}}
\end{aligned}
$$

Demostración. Sea $H=h$ fija y consideremos $h=h_{3}+h_{4}$

$$
h=\underbrace{\left(\frac{1}{2(1+\epsilon)} p_{3}^{2}-\frac{1+\epsilon}{\sqrt{q_{3}^{2}+1 / 4}}\right)}_{h_{3}}+\underbrace{\left(\frac{1}{2(1-\epsilon)} p_{4}^{2}-\frac{1-\epsilon}{\sqrt{q_{4}^{2}+1 / 4}}\right)}_{h_{4}} .
$$

Los valores máximo y mínimo para $q_{3}$ y $q_{4}$ respectivamente se pueden obtener de esta expresión cuando $p_{3}=p_{4}=0$, es decir, cuando la solución pasa por $\mathcal{L}_{1}$; estos valores son

$$
q_{3 \text { máx }}=\sqrt{\left(\frac{1+\epsilon}{h_{3}}\right)^{2}-\frac{1}{4}} \quad \text { y } \quad q_{4 \text { mín }}=-\sqrt{\left(\frac{1-\epsilon}{h_{4}}\right)^{2}-\frac{1}{4}} .
$$


Sustituyendo directamente los valores de $h_{3}$ y $h_{4}$ encontrados en (4.10) tenemos

$$
q_{3 \text { máx }}=\sqrt{\frac{(1+\epsilon)^{2}}{\left(\frac{2 \epsilon}{\sqrt{q_{0}^{2}+1 / 4}}-\frac{(1-\epsilon) h}{2}\right)^{2}}-\frac{1}{4}} \quad y \quad q_{4 \text { mín }}=-\sqrt{\frac{(1-\epsilon)^{2}}{\left(\frac{2 \epsilon}{\sqrt{q_{0}^{2}+1 / 4}}+\frac{(1+\epsilon) h}{2}\right)^{2}}-\frac{1}{4}} .
$$

El movimiento del punto $\mathcal{C} \mathcal{M}$ tiene ecuación $\mathcal{C} \mathcal{M}(\tau)=\frac{1+\epsilon}{2} q_{3}(\tau)+\frac{1-\epsilon}{2} q_{4}(\tau) \mathrm{y}$ alcanza sus puntos máximo y mínimo en $\mathcal{P}$ y $\mathcal{L}_{0}$, es decir cuando $p_{3}+p_{4}=0$. Los puntos extremos deben ser simétricos lo que implica que

$$
-q_{0}=\frac{1+\epsilon}{2} q_{3 \text { máx }}+\frac{1-\epsilon}{2} q_{4 \text { mín }}
$$

Finalmente, sustituyendo los valores de $q_{3 \text { máx }}$ y $q_{4 \text { mín }}$ en la expresión anterior se obtiene el resultado deseado.

4.2.1. Continuación DE ÓRBitas. La solución $\sigma(t)$ encontrada en el Corolario 3 donde $\sigma(0) \in \mathcal{P}$ y $\sigma\left(\tau_{0}\right) \in \mathcal{L}_{0}$, se puede continuar a través de $\mathcal{L}_{0}$ hasta su retorno al plano $\mathcal{P}$. Cuando la solución llega al instante $\tau=2 \tau_{0}$ se cumple $\sigma\left(2 \tau_{0}\right) \in \mathcal{P}$. Y utilizando los Lemas 3.10 y 3.12 identificamos $\sigma(0) \sim \sigma\left(2 \tau_{0}\right)$ y hemos generado una órbita periódica mediante una cadena de orden 1 con periodo $2 \tau_{0}$.

Estas soluciones son periódicas en el sistema binario secundario, independientemente de los periodos del sistema binario primario. Para tener órbitas periódicas del problema completo, debemos considerar los valores de energía tal que generan órbitas periódicas para los cuatro cuerpos. Consideremos el conjunto

$$
\mathfrak{H}=\{h \in(-2,0) \mid T(h)=2 n \pi\}
$$

de valores de energía que genera las órbitas periódicas del problema circular de Sitnikov (clásico) (63).

Para cada $h_{*} \in \mathfrak{H}$, el problema circular de Sitnikov de $2+2$ cuerpos con función Hamiltoniana definida en (4.5) y donde $\epsilon=0$, tiene una órbita periódica simétrica de periodo $n \pi$. De esta forma, podemos ver que para un nivel de energía fijo $h \in(-4,0)$, existe un único elemento $x \in \mu(M)$ tal que $x \in \mathscr{D}$ y tiene la forma $x=\left(h_{*}, h_{*}\right)$ (el conjunto $\mathscr{D} \subset \mu(M)$ se definió en (4.1) $)$. La fibra $\mathscr{T}_{x}$ corresponde a un toro Lagrangiano con generadores idénticos que contiene una solución $\sigma(\tau)$ periódica y simétrica. Esta solución es única salvo por desplazamientos en el tiempo.

Ahora consideremos el conjunto de valores para la energía del problema circular restringido de Sitnikov de $2+2$ cuerpos y definamos el subconjunto

$$
\hat{\mathfrak{H}}=\left\{h \in(-4,0) \mid \frac{h}{2} \in \mathfrak{H}\right\}
$$

El siguiente lema es inmediato

LEMA 4.22 (Jiménez-Pérez). El problema circular restringido de Sitnikov de $2+2$ cuerpos, con parámetro $\epsilon=0$ y energía fija $h$, posee una órbita periódica simétrica de periodo $n \pi$ si y sólo si $h \in \hat{\mathfrak{H}}$. 
Esta familia de órbitas periódicas se puede extender de manera natural mediante la construcción detallada en los párrafos anteriores a una familia de órbitas periódicas para el problema no simétrico donde $\epsilon \neq 0$ es pequeña. Para ello, se utilizará el teorema de la función implícita

TeOrema 4.24 (Jiménez-Pérez). Cada solución periódica simétrica del problema circular restringido de Sitnikov con 2+2 cuerpos se puede extender a una solución periódica no simétrica con colisiones, para $\epsilon \neq 0$ suficientemente pequeña.

Demostración. Utilizando la Proposición 4.23 se tiene que para toda $h \in(-4,0)$ y $\epsilon \in\left(-\epsilon_{0}, \epsilon_{0}\right)$ existe $q_{0}=q_{0}(h, \epsilon)$ tal que la solución $\sigma(t)$ con condiciones iniciales $\sigma(0) \in \mathcal{P}$ pasa por el plano $\mathcal{L}_{0}$. Además, utilizando el Lema 4.17 tenemos que la condición inicial $\sigma_{0}=\sigma(0) \in \mathcal{P}$ que lleva a $\mathcal{L}_{0}$ solo depende de $h$ y de $\epsilon$. En particular si $\epsilon=0$, la solución con condiciones iniciales $\sigma_{0}=\sigma_{0}(h, 0)$ es una solución periódica simétrica si y solo si $h \in \hat{\mathfrak{H}}$.

El valor $\tau_{0}$ es el tiempo que la solución utiliza para ir de $\mathcal{P}$ a $\mathcal{L}_{0}$ está dado explícitamente por

$$
\tau_{0}(h, \epsilon)=\int_{q_{0}}^{q_{3_{\text {máx }}}} \frac{d q_{3}}{\sqrt{\frac{2}{1+\epsilon}\left(\frac{h}{2}+\frac{2}{\sqrt{q_{3}^{2}+1 / 4}}-\epsilon\left(\frac{h}{2}+\frac{2}{\sqrt{q_{0}^{2}+1 / 4}}\right)\right)}}
$$

La función $\tau_{0}(h, \epsilon):(-4,0) \times\left(-\epsilon_{0}, \epsilon_{0}\right) \rightarrow \mathbb{R}$ es continua y diferenciable. Adicionalmente, el Lema 4.20 nos indica que

$$
\left.\frac{\partial \tau_{0}}{\partial \epsilon}(h, \epsilon)\right|_{\epsilon=0} \neq 0
$$

Debemos notar que $\tau_{0}$ depende de $q_{0}$ como parámetro fijo. El teorema de la función implícita nos asegura que localmente existe una función continua $h=h\left(\tau_{0}, \epsilon\right)$ en una vecindad $V_{\delta}$ alrededor del punto $\left(\tau_{0}, 0\right)$ tal que $h\left(\frac{n \pi}{2}, 0\right)=$ $h_{*}$. Para $\epsilon>0$ pequeña, el valor $h_{\epsilon}=h\left(\tau_{0}, \epsilon\right)$ se encuentra dentro de una bola abierta de radio $\delta>0$ es decir $h_{\epsilon} \in\left(h_{*}-\delta, h_{*}+\delta\right)$. Concluimos que para $\epsilon>0$ pequeña, existe un valor $h_{\epsilon}$ en una vecindad de $h_{*}$ tal que, $\tau_{0}\left(h_{\epsilon}, \epsilon\right)=\frac{n \pi}{2}$.

Consideremos el punto $x \in \mathscr{D} \subset \mu(M)$ que toma el valor $x=\left(h_{\epsilon}, h_{\epsilon}\right)$. La fibra $\mathscr{T}_{x}=\mu^{-1}(x)$ contiene una solución $\hat{\sigma}(\tau)$ tal que $\hat{\sigma}(0) \in \mathcal{P}$ toma los valores $\hat{\sigma}(0)=\left(q_{0}, q_{0}, p_{0},-p_{0}\right)$, donde $q_{0}=q_{0}\left(h_{\epsilon}, \epsilon\right)$ y $p_{0}=p_{0}\left(q_{0}\left(h_{\epsilon}, \epsilon\right), h_{\epsilon}, \epsilon\right)$ se obtienen a partir del Lema 4.17y de la Proposición 4.23. Por construcción se tiene que $\hat{\sigma}\left(\frac{n \pi}{2}\right) \in \mathcal{L}_{0}$ y finalmente $\hat{\sigma}(n \pi) \in \mathcal{P}$.

Identificando $\hat{\sigma}(0) \sim \hat{\sigma}(n \pi)$ construimos una solución periódica de periodo $\tau=n \pi$ con rebote elástico en las colisiones y cuya evolución no es simétrica con respecto del plano donde evolucionan los primarios.

NotA 4.13. Es importante ver que estas soluciones para $\epsilon \neq 0$ no se pueden extender a un flujo completo como en el caso $\epsilon=0$. De hecho, en el espacio fase estas soluciones tienen discontinuidades debidas al rebote 
elástico con diferentes masas. A diferencia del problema de Kepler, en este tipo de sistemas se tienen fuerzas externas (el sistema primario) que hace evidente la singularidad a pesar de tener un sistema regularizado.

Nota 4.14. Es posible acotar de manera adecuada el valor del parámetro $\epsilon$ para valores pequeños de $n \in \mathbb{N}$ o de manera equivalente para valores de $h$ que no estén cercanos a cero. Conforme $n$ crece el valor de la energía se acerca asintóticamente a cero y las cotas para el parámetro $\epsilon$ tenderán a cero a una tasa demasiado elevada. Esto podría establecer una obstrucción para poder continuar las órbitas simétricas para valores $n>N \in \mathbb{N}$ suficientemente grandes.

\subsection{CADENAS DE ORDEN MAYOR Y RELACIÓN $n: m$}

Bajo las mismas hipótesis que en la sección anterior, se puede considerar la continuación de órbitas periódicas con energías relativas $h_{3}$ y $h_{4}$ que generen periodos $T\left(h_{3}\right)$ y $T\left(h_{4}\right)$ en relación $n: m$ para $n, m \in \mathbb{N}$ y $n \neq m$. Esto se puede considerar si en el caso $\epsilon=0$ existe una solución periódica que pase por los planos $\mathcal{L}_{0}$ y $\mathcal{P}$ al igual que en el caso 1:1.

Para este caso, se deben cumplir exactamente las mismas hipótesis, sin embargo para calcular específicamente los valores de $q_{0}$ y $p_{0}$ así como las cotas para $\epsilon$ se deberan refinar las demostraciones.

En general tenemos que para toda $h \in(-4,0)$ y para $\epsilon=0$ el problema circular de Sitnikov de $2+2$ cuerpos tiene órbitas periódicas donde los tiempos de retorno $T\left(h_{3}\right)$ y $T\left(h_{4}\right)$ están en relación $n: m$ para $n, m \in \mathbb{N}$. Para poder extender estas órbitas mediante el método utilizado en este capítulo es necesario que pasen por los planos $\mathcal{L}_{0}$ y $\mathcal{P}$. Véase las figuras 4.8 y 4.9
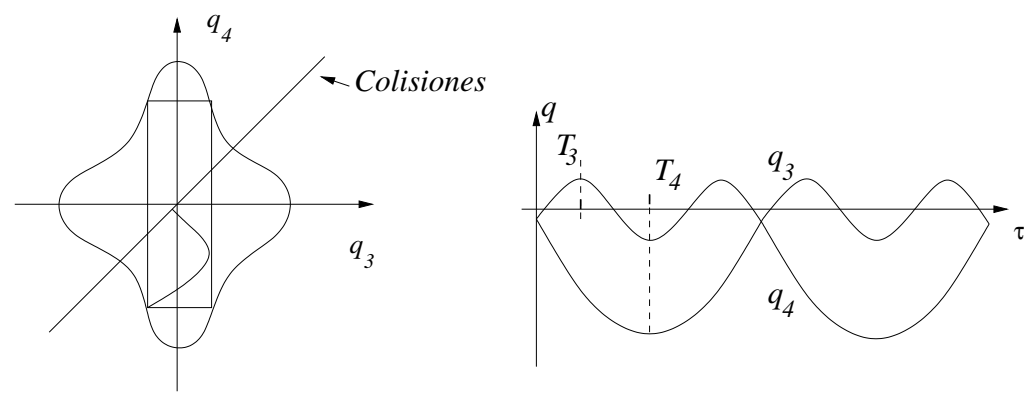

FigurA 4.8. Órbita generatriz con relación 2:1. 

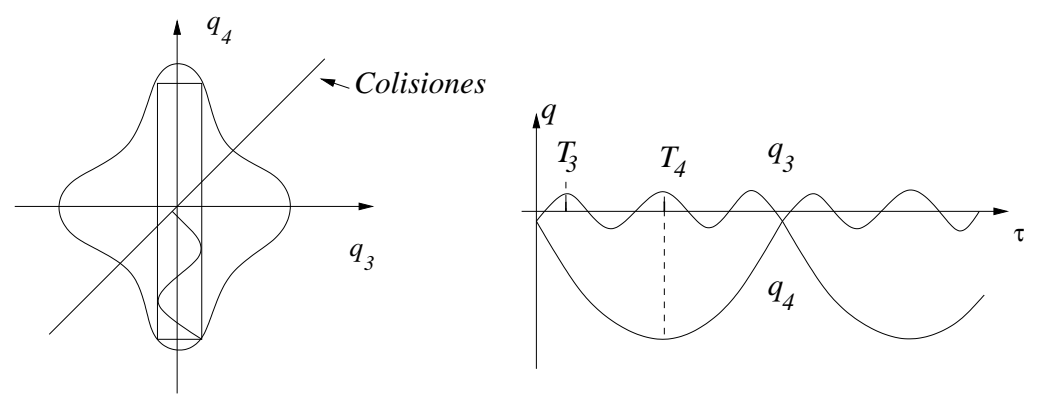

FigurA 4.9. Órbita generatriz con relación 3:1. 



\section{CAPÍTULO 5}

\section{CONCLUSIONES Y PERSPECTIVAS.}

En este trabajo se ha presentado una configuración original de un problema restringido de 4 cuerpos de la mecánica celeste. La configuración propuesta consiste en una extensión del problema de Sitnikov donde se tienen dos cuerpos primarios que evolucionan en órbitas Keplerianas y dos cuerpos secundarios que evolucionan en la línea recta, perpendicular al plano de los cuerpos primarios, y que pasa por su centro de masa.

El estudio cuidadoso del caso circular (integrable), sin atracción gravitacional entre los secundarios, establece una base sólida para comprender el comportamiento del sistema en algunos casos más generales. En particular, se hizo un extenso estudio acerca del intercambio de momento que permitirá predecir la dinámica de los secundarios en el caso con masas positivas pequeñas.

A continuación puntualizaremos las aportaciones que ofrece este trabajo de investigación y daremos algunas conclusiones basadas en el punto de vista del autor.

\subsection{Regularización DE COLISIONES.}

La regularización de colisiones presentada en este trabajo está basada en la transformación de Euler para colisiones binarias. (Básicamente, la transformación de Euler es la versión unidimensional de la regularización de Levi-Civita.) Esta regularización se generaliza de manera natural a sistemas de $N+2$ cuerpos donde los secundarios evolucionan sobre una recta perpendicular al plano donde evolucionan los $N$ cuerpos primarios (Teorema 2.14). Los $N$ primarios deberán estar en un equilibrio relativo y el potencial debe ser simétrico con respecto a la recta donde evolucionan los secundarios. En 
particular, se puede hacer una extensión inmediata del problema de Sitnikov de $3+1$ cuerpos propuesto por Soulis, Papadakis y Bountis [95, 2008] al caso con $3+2$ cuerpos. Y en general al problema de $N+2$ cuerpos con $N$ primarios en equilibrio relativo sobre un polígono regular de $N$ lados.

Una característica importante en la función Hamiltoniana del problema estudiado en este trabajo, es que las masas de los secundarios son arbitrarias (pero del mismo orden) y tienen una relación constante. La regularización refleja esa relación entre las masas de los secundarios y la preserva cuando se obtiene el caso límite $m_{i} \rightarrow 0$. Después de obtener el límite el sistema se desacopla, pero los parámetros introducidos en la relación de masas se preservan en la función Hamiltoniana y en las ecuaciones de movimiento. Este hecho será de gran impacto para estudiar el intercambio de momento que sufren los secundarios durante las colisiones. Existen artículos donde se realiza la regularización de colisiones binarias bajo circustancias relativamente parecidas [98, sin embargo, el autor no ha encontrado aplicaciones directas al intercambio de momento.

\subsection{COORDENADAS ACCIÓN-ÁNGULO Y SOLUCIÓN ANALÍTICA.}

Las coordenadas acción-ángulo son una herramienta muy utilizada en sistemas Hamiltonianos para determinar estabilidad de soluciones y caoticidad de sistemas, entre otras características dinámicas. En particular, para sistemas integrables con dos grados de libertad, las coordenadas acciónángulo nos permiten determinar secciones de Poincaré bidimensionales 61 y facilitan el estudio del sistema bajo métodos perturbativos 61, 77.

En este trabajo se encuentran las coordenadas acción-ángulo (del problema circular) en forma analítica mediante funciones e integrales elípiticas de Jacobi. Esto permitirá hacer excelentes aproximaciones númericas para la aplicación de Poincaré (aún en el problema de Sitnikov clásico) ya que las coordenadas podrán escribirse como cocientes de funciones $\theta_{i}$ de Jacobi o como series de Eisenstein [59, 73]. Estos métodos pueden dar mejores aproximaciones numéricas que las funciones de Bessel [13] y sería muy interesante estudiar la velocidad de convergencia en ambos casos. Como una alternativa, se pueden hacer estudios comparativos (para el problema de Sitnikov clásico) y algunas extensiones (en el caso del problema de Sitnikov de 2+2) a los trabajos de Liu y Sun 62, Perdios y Markellos [84, Dvorak 31, Jalali y Pourtakdoust [46, Chesley [21, Jiménez-Lara y Buendía 47, entre otros.

Como hemos visto, el sistema asociado al problema circular restringido de Sitnikov con $2+2$ cuerpos se desacopla y puede ser estudiado como un producto cartesiano de dos problemas de Sitnikov clásicos con diferentes escala de tiempo. Entonces, la solución analítica corresponde es una extensión de la solución encontrada por [65, detallada por Belbruno, Llibre y Ollé 8 y reescrita en integrales elípticas canónicas de Jacobi en [25]. Aquí se da una 
demostración más directa utilizando propiedades de las integrales elípticas y se hace énfasis en mantener la escala de tiempo que proporcionará el intercambio de energía en las colisiones.

\subsection{TRANSFERENCIA DE MOMENTO.}

La transferencia de momento es, sin duda, la parte más interesante de este trabajo. Cuando se tiene un problema de $N+2$ cuerpos con colisiones binarias entre dos infinitesimales, el problema puede estudiarse como dos problemas de $N+1$ cuerpos independientes 66. Si el movimiento de los infinitesimales no es rectilíneo en la colisión entonces se considera un cambio en las órbitas que puede ocurrir de diversas manera. Este método es utilizado por Bolotin, McKay [1, 12 y por Marco, Niederman 66 para construir órbitas periódicas sin colisión que se encuentran en una vecidad de una órbita periódica con colisión. Las órbitas construidas de esta forma se conocen como órbitas de shadowing y se considera que los infinitesimales tienen masas (infinitesimal) iguales. Un caso más general es cuando las masas de los cuerpos que colisionan no son iguales (ya sea del mismo orden o no) y la colisión puede o no ser rectilínea. Para estimar la continuación de las trayectorias es necesario conocer el momento angular del sistema que puede generar una dispersión en las órbitas después de la colisión. A este fenómeno se le conoce como scattering [22, 41] y se utiliza en mecánica celeste para diseño de misiones "asistidas" que utilizan la fuerza de gravedad de los planetas con masa muy grande (Jupiter o Saturno) para impulsar sondas espaciales hacia los exoplanetas 41.

En este trabajo se estudia la transferencia de momento sin considerar atracción entre los secundarios y se extienden las soluciones mediante rebote elástico. Ambas condiciones generan una matriz de transición entre los momentos de los secundarios y genéricamente existirá un intercambio entre sus energías relativas (Lemas 3.9 y 3.10). Esto implica que al pasar por una colisión, la solución cambia de un toro invariante a otro toro invariante excepto si la solución pasa por el plano $\left\{p_{3}+p_{4}=0\right\} \cap \Delta$ (Lema 3.11). Ambos toros se encuentran dentro de la misma superficie de energía, y si ésta es no compacta, se pueden presentar escapes mediante una sucesión adecuada de colisiones.

Adicionalmente, el autor conjetura que dada la sensibilidad que tienen los valores de los periodos $T\left(k_{i}\right)$ con respecto a variaciones en los módulos

$$
k_{i}=\frac{\sqrt{2+\frac{h_{i}}{1 \pm \epsilon}}}{2},
$$

se puede tener comportamiento caótico o cuasi-aleatório de los "saltos" que experimenta la solución durante las colisiones. Un comportamiento no esperado para un problema integrable. 


\subsection{FOLIACIÓN DEL ESPACIO FASE.}

La foliación del espacio fase por niveles de energía constante se realizó mediante la acción Hamiltoniana de grupos de Lie y una aplicación momento. En este sentido, se ofrecen dos breves resultados del autor (Proposiciones 3.19 y 3.20) para determinar la imagen de las superficies de energía constante bajo la aplicación momento. Los resultados se pueden obtener utilizando el Teorema de Liouville-Arnold y el Teorema de la función implícita, sin embargo, los últimos resultado son locales, mientras que la técnica de acciones Hamiltonianas y aplicaciones momento es, en cierta manera, global.

El análisis de la imagen de la aplicación momento nos permite encontrar dos valores donde se bifurca la topología de las superficies de energía constante. Estos valores son $h=-2(1+\epsilon)$ y $h=-2(1-\epsilon)$ y corresponde a la posibilidad de escapes de alguno de los secundarios. Ellos coinciden con el problema donde se tiene atracción entre los cuerpos secundarios y esos resultados serán presentados en un trabajo posterior. Los dos niveles de energía mencionados anteriormente, poseen un punto fijo (dos puntos fijos que se identifican por la restricción $q_{3}>q_{4}$ ) de tipo centro-silla al infinito. Estos puntos fijos permiten demostrar la no integrabilidad meromorfa del problema de Sitnikov de $2+2$ cuerpos como se detalla en [52. La técnica que se utiliza es la reducción del sistema a un plano invariante (el centro) y se aplica una equivalencia entre la Teoría de Galois diferencial y la Teoría de Melnikov.

\subsection{CONTINUACIÓN DE ÓBITAS PERIÓDICAS.}

Durante el estudio de la transferencia de energía y de momento se encontró evidencia de posibles comportamientos cuasi-aleatorios cuando la solución salta de un toro invariante a otro durante una colisión. La acción natural es encontrar las condiciones que deben cumplir las soluciones para mantenerse en un mismo toro invariante y dadas esas condiciones tratar de determinar si existen órbitas periódicas que se preserven cuando los valores de las masas son diferentes.

En este sentido, se encontró que la única familia de soluciones que puede extenderse de esa forma son las soluciones simétricas (con respecto del plano del sistema primario). Para demostrar la existencia de este tipo de órbitas periódicas, se utilizó una variación de la continuación de órbitas periódicas de Poincaré [74, 75] aplicando dos veces el teorema de la función implícita. A diferencia de los problemas clásicos donde se aplica el método de continuación de Poincaré, nuestras soluciones siguen siendo órbitas con colisiones (por ser un sistema rectilíneo). Una de las aplicaciones inmediatas es en el doble péndulo desacoplado con masas diferentes que tiene un comportamiento equivalente. 
Finalmente, parece interesante la búsqueda de soluciones periódicas que pasen por más de una colisión antes de cerrar la órbita. Esto se propone como un trabajo posterior utilizando un mapeo de Poincaré y propiedades de las funciones e integrales elípticas de Jacobi.

\subsection{Nuevas POSIBILIDAdES DE INVESTIGACIÓN.}

El estudio del problema circular de Sitnikov con $2+2$ cuerpos marca diferentes caminos para continuar con las investigaciones dentro de la mecánica celeste. Apenas se vislumbra "la punta del iceberg", ya que en general, el problema clásico de Sitnikov es un problema difícil y complejo. Por lo tanto, cada extensión de este problema da lugar a muchas interrogantes. En las siguientes secciones daremos algunas perspectivas y algunos problemas abiertos que son interesantes por sí mismos. Varios de los problemas propuestos a continuación han surgido de las pláticas con diversos investigadores y en particular en las discusiones con los miembros del jurado de este trabajo doctoral.

5.6.1. UnA EXTENSIÓN DEL ACELERADOR DE FERMI. El acelerador de Fermi es un sistema dinámico originalmente propuesto por Enrico Fermi 32 para describir la aceleración de los rayos cósmicos mediante colisiones con un campo magnético que depende del tiempo. El modelo original de Fermi fue estudiado y modificado por diversos autores y desde diferentes perspectivas, una de las cuales llevó al conocido modelo de Fermi-Ulam (FUM por sus siglas en inglés). El modelo FUM consiste en un mapeo obtenido como la idealización del movimiento unidimensional de una pelota rebotando entre una pared fija y otra que se mueve de manera oscilatoria 61. Sea $v_{n}$ la velocidad de la pelota, normalizada a la velocidad máxima de la pared oscilatoria y sea $\theta_{n}$ la fase de dicha pared justo antes de la $n$-ésima colisión con la pelota. El modelo FUM tiene el mapeo siguiente

$$
\begin{aligned}
& v_{n+1}=\left|v_{n}+\sin \left(\theta_{n}\right)\right|, \\
& \theta_{n+1}=\theta_{n}+\frac{2 \pi M}{v_{n+1}},
\end{aligned}
$$

donde $M$ es la distancia normalizada entre las paredes y se considera la magnitud absoluta de $v_{n+1}$.

Este es un modelo totalmente simplificado en el cual se introduce una superficie fija de sección en $x=0$ que no es relevante ya que la velocidad de la pelota entre las paredes es constante, y solo se modifica en cada colisión con la pared oscilatoria.

Un modelo más sofisticado consiste en considerar el problema circular de Sitnikov con $2+2$ cuerpos en donde la razón de masas entre los secundarios

\footnotetext{
${ }^{1}$ El autor agradece al Dr. Arturo Olvera por la sugerencia de este modelo como una herramienta para determinar escapes en el problema circular de Sitnikov con $2+2$ cuerpos.
} 
cambia de orden (tiende a cero). Este caso representa el límite cuando $\epsilon \rightarrow 1$ y corresponde a una nueva versión del caso restringido en donde uno de los cuerpos (el de mayor masa) evoluciona como el problema de Sitnikov clásico mientras que el cuerpo de menor masa viaja como una partícula de prueba bajo la atracción de los primarios y colisiona con el cuerpo de masa media sin perturbar su movimiento.

Para obtener el modelo consideremos el problema de Sitnikov con $2+2$ cuerpos, escribiremos las masas de los secundarios de la forma

$$
m_{3}=m \mu \quad \text { y } \quad m_{4}=m(1-\mu),
$$

para $0<m \ll 1 / 2$ y $\mu \in(0,1 / 2)$. Las ecuaciones de movimiento se obtienen mediante

$$
\begin{aligned}
& m_{3} \ddot{q}_{3}=-\frac{m_{3} q_{3}}{\left(q_{3}^{2}+1 / 4\right)^{\frac{3}{2}}}-\frac{m_{3} m_{4}}{\left(q_{3}-q_{4}\right)^{2}} \\
& m_{4} \ddot{q}_{4}=-\frac{m_{4} q_{4}}{\left(q_{4}^{2}+1 / 4\right)^{\frac{3}{2}}}+\frac{m_{3} m_{4}}{\left(q_{3}-q_{4}\right)^{2}} .
\end{aligned}
$$

Cancelando los términos de las masas que se pueden simplificar tenemos

$$
\begin{aligned}
& \ddot{q}_{3}=-\frac{q_{3}}{\left(q_{3}^{2}+1 / 4\right)^{\frac{3}{2}}}-m \frac{1-\mu}{\left(q_{3}-q_{4}\right)^{2}}, \\
& \ddot{q}_{4}=-\frac{q_{4}}{\left(q_{4}^{2}+1 / 4\right)^{\frac{3}{2}}}+m \frac{\mu}{\left(q_{3}-q_{4}\right)^{2}} .
\end{aligned}
$$

Considerando el límite cuando $\mu \rightarrow 0$ la expresión (5.6) se convertirá en la ecuación de movimiento del problema circular de Sitnikov clásico. Por otra parte, la expresión (5.5) será la ecuación del cuerpo con masa $m_{3}$ de orden mínimo que llamaremos el terciario. La función Hamiltoniana para el terciario es

$$
H=\frac{1}{2} p^{2}-\frac{1}{\sqrt{q_{3}^{2}+1 / 4}}-\frac{m}{\left|q_{3}-\sigma(t)\right|},
$$

donde $\sigma(t)$ es una solución acotada del problema de Sitnikov clásico.

Si se cumple que $\left|q_{3}-\sigma(t)\right|>N$ para alguna $N \in \mathbb{N}$ suficientemente grande, entonces el último término en (5.7) es irrelevante y podemos aproximar el mapeo únicamente con el intercambio de momento durante la colisión, como se realiza con el modelo FUM del acelerador de Fermi. Sin embargo, la dificultad principal en el nuevo modelo es determinar el tiempo entre dos colisiones sucesivas para estimar el intercambio de energía.

Cuando la distancia $\left|q_{3}-\sigma(t)\right|>N$ es suficientemente grande, se puede aproximar la velocidad de la $n$-ésima colisión como una pequeña variación de la velocidad de la $n$-ésima expulsión, es decir

$$
\left(v_{c o l}\right)_{n}=-\left(v_{\text {exp }}\right)_{n}+\delta,
$$

donde $0 \leq \delta$ es pequeña y depende de la solución particular del secundario. $\mathrm{Al}$ instante posterior a la colisión la velocidad de expulsión será el negativo 
de la velocidad de colisión más la velocidad que agrega el secundario en ese mismo instante. Por lo tanto tendremos

$$
\begin{aligned}
\left(v_{\text {exp }}\right)_{n+1} & =-\left(v_{c o l}\right)_{n}+V\left(\psi_{n+1}\right), \\
& =\left(v_{\text {exp }}\right)_{n}-\delta+V\left(\psi_{n+1}\right),
\end{aligned}
$$

donde $V\left(\psi_{n+1}\right)$ es la velocidad del secundario en el momento de la colisión. A partir de las expresiones anteriores, el mapeo puede estimarse únicamente con la velocidad de expulsión del terciario, por lo que eliminaremos el subíndice y escribiremos $v_{n}=\left(v_{\text {exp }}\right)_{n}$.

La ecuación en diferencias para el movimiento del cuerpo terciario también estará determinada por el movimiento del secundario. Para ello, consideremos una solución periódica del secundario con energía relativa $\hat{h}<0$ dada por $\hat{\sigma}(t)=(Q(\theta), P(\theta))$ con fase $\theta=\frac{1}{\Omega} t$ y periodo $2 \pi$. Podemos determinar las ecuaciones de movimiento para el terciario mediante

$$
\begin{aligned}
v_{n+1} & =v_{n}+P\left(\psi_{n}\right), \\
\psi_{n+1} & =\left(\psi_{n}+\Delta \psi\right) \bmod 2 \pi .
\end{aligned}
$$

donde $\Delta \psi$ indicará el instante en que se realizará la siguiente colisión.

Determinar el instante de la siguiente colisión implica resolver la ecuación $Q(\theta)-q(\theta)=0$ para $\theta$, donde ambas funciones son soluciones del problema de Sitnikov clásico. Específicamente, debemos resolver

$$
k \frac{\operatorname{sn}(\theta, k) \operatorname{dn}(\theta, k)}{1-2 k^{2} \operatorname{sn}^{2}(\theta, k)}-\kappa \frac{\operatorname{sn}(\theta, \kappa) \operatorname{dn}(\theta, \kappa)}{1-2 \kappa^{2} \operatorname{sn}^{2}(\theta, \kappa)}=0,
$$

donde $k=\frac{\sqrt{2+\hat{h}}}{2}$ es el valor fijo que determina la evolución del secundario y $\kappa=\frac{\sqrt{2+h}}{2}$ se modifica en cada colisión mediante la ganancia o pérdida de momento del cuerpo terciario. Finalmente $\Delta \psi$ estará dada por dos soluciones consecutivas de la expresión (5.11).

Una aproximación más plausible consiste en considerar la distancia del secundario a uno de los primarios dada por $r_{s}=\sqrt{Q^{2}+1 / 4}$ y la del terciario al mismo primario mediante $r_{t}=\sqrt{q^{2}+1 / 4}$. Si $Q-q=0$ esto indica que las tangentes de los ángulos $\alpha$ y $\beta$ son iguales (véase la Figura 5.11).

Considerando $\tan \alpha=2 q$ y $\tan \beta=2 Q$ tenemos que $Q-q=0$ implica $\tan \alpha-\tan \beta=0$. Además

$$
\tan (\alpha-\beta)=\frac{\tan \alpha-\tan \beta}{1+\tan \alpha \tan \beta},
$$

de donde se obtiene

$$
Q-q=\frac{1}{2} \tan (\alpha-\beta)(1+\tan \alpha \tan \beta) .
$$

En este caso nos interesan las soluciones que cumplen $\tan (\alpha-\beta)=0$ que corresponden a $Q-q=0$, ya que $\alpha, \beta \in(-\pi / 2, \pi / 2)$. Consideremos $\alpha=2 \theta$ 


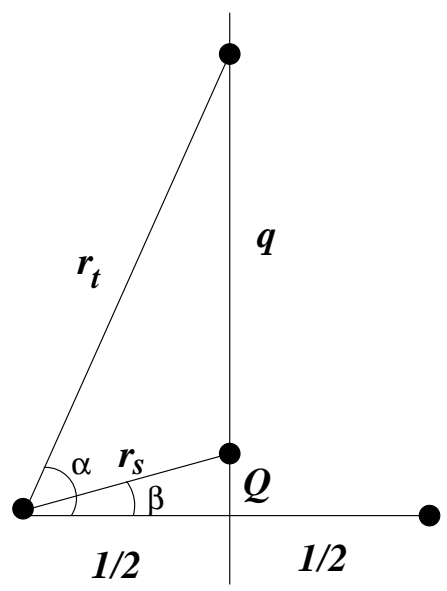

Figura 5.1. Nuevas coordenadas para el problema circular de Sitnikov de $2+2$ como un acelerador de Fermi modificado.

y $\beta=2 \phi$, entonces

$$
\begin{aligned}
\tan (\alpha-\beta) & =\tan (2(\theta-\phi)), \\
& =\frac{2 \sin (\theta-\phi) \cos (\theta-\phi)}{1-2 \sin ^{2}(\theta-\phi)} .
\end{aligned}
$$

Esto permite construir una relación del tipo

$$
\hat{k} \frac{\operatorname{sn}(\theta-\phi, \hat{k}) \operatorname{dn}(\theta-\phi, \hat{k})}{1-2 \hat{k}^{2} \operatorname{sn}^{2}(\theta-\phi, \hat{k})}=0,
$$

para $\hat{k}=\hat{k}(k, \kappa)$. Esta relación tiene solución real $\operatorname{si} \operatorname{sn}(\theta-\phi, \hat{k})=0$ que indica que $\theta-\phi=2 n K(\hat{k})$, donde $K(\hat{k})$ es la integral elíptica completa de primera especie. Finalmente, $\Delta \psi$ está determinada por $K(\hat{k})$ y $\hat{k}$ se debe obtener a partir de la velocidad (o del momento) para cada iteración. Entonces el modelo se establece mediante tres ecuaciones de la forma

$$
\begin{aligned}
v_{n+1} & =v_{n}+V\left(\psi_{n}\right), \\
\psi_{n+1} & =\psi_{n}+\Delta \psi(K(\hat{k})) \\
\hat{k}_{n+1} & =\frac{\sqrt{2 \pm L\left(0, v_{n+1}\right)}}{2},
\end{aligned}
$$

donde $V\left(\psi_{n}\right)=P\left(\psi_{n}\right)$ y $L\left(0, v_{n+1}\right)$ es la función Lagrangiana obtenida a partir de la transformación de Legendre de (5.7), para $m=0$.

Uno de los primeros objetivos es determinar la formulación más adecuada para este problema. El acelerador de Fermi se ha estudiado principalmente en su formulación Lagrangiana, sin embargo, el intercambio de momento sugiere utilizar la formulación Hamiltoniana para nuestro modelo. Parece evidente que la identidad $V\left(\psi_{n}\right)=P\left(\psi_{n}\right)$ requiere la relación de masas como parámetro y esto puede determinar cuál será la formulación adecuada. 
Otro comentario acerca de este acercamiento está relacionado con la ecuación

$$
\hat{k}_{n+1}=\frac{\sqrt{2 \pm L\left(0, v_{n+1}\right)}}{2},
$$

que tiene relación directa con la energía del problema. Esta ecuación será conjugada a la expresión del tiempo dada por $\Delta \psi$. Para establecer que el mapeo sea Hamiltoniano, tal vez sea adecuado trabajar directamente con la función Hamiltoniana $H$ o la Lagrangiana $L$ en lugar del módulo $\hat{k}$. 



\section{APÉNDICE A}

\section{Grupos y Álgebras DE LIE}

\section{A.1 Grupos De LiE.}

Definición A.1. Un Grupo de Lie es una variedad de dimensión finita sobre la cual se define una estructura de grupo, donde las operaciones de grupo están dadas por las aplicaciónes diferenciables

$$
\begin{aligned}
& G \times G \rightarrow G, \quad \text { y } \quad G \rightarrow G, \\
& (g, h) \mapsto g h, \quad g \mapsto g^{-1} .
\end{aligned}
$$

Como este trabajo se basa en variedades diferenciables sobre el campo $\mathbb{R}$ de los números reales, nos enfocaremos en los grupos de Lie reales; se pueden definir las propiedades para grupos de Lie con estructura compleja de manera análoga.

Todo grupo de Lie $G$ genera de manera natural un álgebra de Lie denotada por $\mathfrak{g}=\operatorname{Lie}(G)$. Dicha álgebra puede obtenerse como la linelización de $G$ alrededor del elemento identidad del grupo y cuya estructura queda determinada por $G$. Para ello, existen diversas construcciones equivalentes.

Notación. Para cada $g_{0} \in G$ denotamos por $L_{g_{0}}$ la función definida por

$$
g \mapsto L_{g_{0}} g:=g_{0} g,
$$

que es llamada una translación a la izquierda dada por $g_{0}$ y de la misma forma denotaremos por $R_{g_{0}}$ a la función

$$
g \mapsto R_{g_{0}} g:=g g_{0},
$$

que llamaremos una translación a la derecha. Para cada $g_{0}$, las aplicaciones $L_{g_{0}}$ y $R_{g_{0}}$ son difeomorfismos de $\mathrm{G}$ en si mismo. Ellas pueden utilizarse también para desplazar campos vectoriales $X$ dados sobre $G$. Esto hace 
posible los conceptos de campos vectoriales invariantes por la derecha y por la izquierda sobre $G$.

Definición. Un campo vectorial $X \in \mathfrak{X}(G)$ se dice que es invariante izquierdo si se cumple

$$
\left(L_{g_{0}}\right)_{*} X=X \quad \forall g_{0} \in G .
$$

El espacio de todos los campos vectoriales sobre $G$ que son invariantes izquierdos y que denotamos por $\mathfrak{X}_{L}(G)$, es un subespacio vectorial de $\mathfrak{X}(G)$, y se puede probar que es un subálgebra de Lie del álgebra de Lie $\mathfrak{X}(G)$. (Es importante notar que un álgebra de Lie puede tener subálgebras que no sean subálgebras de Lie y por lo tanto la frase anterior no es redundante.)

Para poder definir un subgrupo de Lie primero debemos recordar algunas propiedades de una subvariedad diferenciable. Sea $N$ una variedad diferenciable de dimensión $n$. Una subvariedad $M \subset N$ de dimensión $m$ es un subconjunto que cumple que para cada $x \in M$ existe una vecindad $V(x) \subset N$ tal que la intersección $M \cap V(x)$ puede describirse de alguna de las siguientes formas:

- por funciones diferenciables de manera paramétrica

$$
x_{i}=\phi_{i}\left(t_{1}, \cdots, t_{m}\right), \quad(i=1, \cdots, n) ;
$$

- por ecuaciones de la forma

$$
f\left(x_{1}, \cdots, x_{n}\right)=0, \quad(i=1, \cdots, n-m) ;
$$

donde todas las funciones y ecuaciones son diferenciables.

Definición. Un subgrupo $H$ de un grupo de Lie $G$ se dice que es un subgrupo de Lie si es una subvariedad de la variedad subyacente de $G$.

Desde el punto de vista topológico y de la geometría diferencial todo subgrupo $H$ de un grupo de Lie $G$ se ve idéntico en la identidad que en cualquier $h \in H$ ya que dichos grupos son transformados en sí mismos mediante translaciones por $h$ que es un difeomorfismo de la variedad $G$. Por lo tanto, para verificar que un subgrupo $H$ es un subgrupo de Lie es suficiente establecer que sea una subvariedad en alguna vecindad de la identidad.

Definición. Sean $G$ y $H$ dos grupos de Lie. Una aplicación $f: G \rightarrow H$ es un homomorfismo si se da de manera simultánea que es un homomorfismo de grupos y a la vez una aplicación diferenciable. Un homomorfismo $f$ : $G \rightarrow H$ se dice que es un isomorfismo si existe una aplicación inversa $f^{-1}$ : $H \rightarrow G$, es decir, si $f$ es al mismo tiempo un isomorfismo de grupos y un difeomorfismo de variedades.

Definición. Un homomorfismo $\alpha$ de un grupo de Lie $G$ sobre el grupo de difeomorfismos $\operatorname{Diff}(M)$ sobre una variedad diferenciable $M$ se dice que es una acción sobre $M$ si la aplicación

$$
\begin{aligned}
G \times M & \rightarrow M, \\
(g, x) & \mapsto \alpha(g) x
\end{aligned}
$$

es diferenciable. 
Claramente, la composición de una acción $\alpha: G \rightarrow \operatorname{Diff}(M)$ y de un homomorfismo $f: H \rightarrow G$ es una acción del grupo de Lie $H$ sobre la variedad $M$.

\section{A.2 Álgebras de LiE.}

Las álgebras de Lie surgen de la teoría de Lie al estudiar problemas en grupos de Lie de manera local, lo que reduce el estudio a problemas en álgebras de Lie y de manera consecuente a problemas de álgebra lineal. A cada grupo de Lie se le asocia un álgebra de Lie sobre los números reales o complejos y se establece una correspondencia entre los subgrupos analíticos del grupo de Lie y las subálgebras de su álgebra de Lie, en la cual los subgrupos invariantes corresponden a ideales, subgrupos abelianos a álgebras abelianas, etc. La teoría de las álgebras de Lie se puede considerar como un campo de estudio independiente y autocontenido.

Definición Un espacio vectorial $\mathfrak{g}$ sobre el campo $K$ se denomina un álgebra de Lie si se define un producto corchete de Lie que es una aplicación $K$-bilineal

$$
[\cdot, \cdot]: \mathfrak{g} \times \mathfrak{g} \rightarrow \mathfrak{g}
$$

que satisface

1. la propiedad antisimétrica dada por $[X, X]=0$ para todo $X \in \mathfrak{g}$,

2. la identidad de Jacobi definida por

$$
[[X, Y], Z]+[[Y, Z], X]+[[Z, X], Y]=0, \quad \forall X, Y, Z \in \mathfrak{g} .
$$

Si $\mathfrak{g}$ es un espacio de dimensión finita con una base $\left(X_{1}, X_{2}, \cdots, X_{n}\right)$, es suficiente conocer los valores del corchete de Lie $\left[X_{i}, X_{j}\right]$ para cada par de elementos de la base, para determinar el comportamiento del espacio total. En otras palabras, se deben conocer los coeficientes $c_{i, j}^{k}$ para $i, j, k \in$ $\{1, \cdots, n\}$ para las expresiones

$$
\left[X_{i}, X_{j}\right]=\sum_{k=1}^{n} c_{i, j}^{k} X_{k} .
$$

Estos coeficientes se conocen como las constantes de estructura de $\mathfrak{g}$ relativas a la base $\left(X_{1}, X_{2}, \cdots, X_{n}\right)$.

En particular podemos considerar a los espacios vectoriales sobre $K$ como álgebras de Lie cuando se les asigna el corchete de Lie trivial

$$
[X, Y]=0 \quad \text { para todo } X, Y \in \mathfrak{g} .
$$

En estos casos las álgebras de Lie son llamadas conmutativas o abelianas.

Otro ejemplo de álgebras de Lie surge cuando consideramos un álgebra asociativa genérica $\mathfrak{A}$ sobre un campo $K$ y definimos el corchete de Lie de dos elementos en el álgebra de la siguiente manera

$$
[X, Y]:=X Y-Y X \quad \text { para todo } X, Y \in \mathfrak{A} \text {. }
$$


Al considerar el espacio de matrices cuadradas de dimensión $n \times n$ como álgebra $A=M_{n \times n}(K)$ y proveerla con este corchete de Lie obtenemos un álgebra de Lie conocida como $\mathfrak{g l}_{n}(K)$ que corresponde al espacio tangente a la identidad del grupo general lineal $G L_{n}(K)$ considerado como grupo de Lie.

Ejemplo 1. El álgebra

$$
\left[X_{1}, X_{2}\right]=X_{3},\left[X_{2}, X_{3}\right]=X_{1},\left[X_{3}, X_{1}\right]=X_{2}
$$

es un álgebra de Lie real con tres elementos $(r=3)$. Dicha álgebra corresponde al producto vectorial de $\mathbb{R}^{3}$ y puede interpretarse como el momento angular en tres dimensiones, $\mathfrak{s o}(3)$.

Ejemplo 2. El álgebra

$$
\left[X_{1}, X_{2}\right]=X_{3},\left[X_{2}, X_{3}\right]=-X_{1},\left[X_{3}, X_{1}\right]=X_{2}
$$

es también un álgebra de Lie real con tres elementos $(r=3)$. Debemos notar que el segundo elemento tiene un signo negativo que difiere del caso anterior, este caso corresponde al álgebra de Lorentz en dimensión $2+1, \mathfrak{s o}(2,1)$.

Definición. Consideremos una $\mathbb{K}$-álgebra $A$ arbitraria y no necesariamente asociativa, una derivación $D: A \rightarrow A$ es una aplicación lineal en $\mathbb{K}$ que cumple la regla de Leibnitz

$$
D(X, Y)=(D X) Y+X(D Y) \quad \text { para todo } X, Y \in A \text {. }
$$

Consideremos el conjunto de todas las derivaciones en $A$ como el subconjunto de las aplicaciones lineales $\mathscr{L}(A ; \mathbb{K})$ sobre $A$ y con valores en $\mathbb{K}$ que definiremos por

$$
\mathfrak{D}(A)=\{D \in \mathscr{L}(A) \mid D(X, Y)=(D X) Y+X(D Y), \quad X, Y \in A\} .
$$

Adicionalmente, consideremos el operador $\left[D_{1}, D_{2}\right]:=D_{1} D_{2}-D_{2} D_{1}$ como un corchete de Lie, entonces el conjunto $\mathfrak{D}(A)$ forma un álgebra de Lie llamada el álgebra de derivaciones sobre $A$.

Recordemos que el conjunto de funciones diferenciables definidas sobre una variedad $M$ con imagen en $\mathbb{K}$ forma un álgebra que denotaremos por $\mathfrak{F}(M)$. Utilizando la idea del párrafo anterior, podemos considerar el álgebra de Lie $\mathfrak{D}(\mathfrak{F}(M))$ de las derivaciones del conjunto de funciones diferenciables sobre $M$. Entonces se tiene el siguiente resultado (véase [1, p. 83)

TeOrema A.25. El espacio vectorial $\mathfrak{X}(M)$ de todos los campos vectoriales diferenciables sobre una variedad diferenciable $M$, es isomorfo al espacio de derivaciones $\mathfrak{D}(\mathfrak{F}(M))$ como espacios vectoriales sobre el campo $\mathbb{K}$.

El isomorfismo se define al asignar a un campo vectorial $X$ sobre $M$, la derivación $L_{X}$ definida por

$$
L_{X} f(m)=\left\langle X_{m},(d f)_{m}\right\rangle \quad f \in \mathfrak{F}(M), m \in M .
$$

$L_{X} f$ es llamada la derivada de Lie de $f$ relativa a $X$ y debido a las consideraciones locales se puede escribir de manera alternativa como $L_{X} f(m)=$ $L_{X_{m}} f$. El isomorfismo del teorema proporciona una estructura de álgebra de 
Lie sobre $\mathfrak{X}(M)$, donde $[X, Y]$ es un campo vectorial determinado de manera única mediante

$$
L_{[X, Y]}=\left[L_{X}, L_{Y}\right] \quad X, Y \in \mathfrak{X}(M) .
$$

Si consideramos un difeomorfismo $F: M \rightarrow M^{\prime}$ entonces $F^{*} X \in \mathfrak{X}\left(M^{\prime}\right)$ es considerado como la imagen de $X \in \mathfrak{X}(M)$. La aplicación $F^{*}$ es compatible con el corchete de Lie y es un homomorfismo de álgebras de Lie; es decir, $F^{*}$ es $\mathbb{K}$-lineal y cumple

$$
F^{*}[X, Y]=\left[F^{*} X, F^{*} Y\right] \quad X, Y \in \mathfrak{X}(M) .
$$

\section{A.3 Álgebras de Lie de Grupos De LIE.}

Ya comentamos que históricamente, las álgebras de Lie nacieron como aproximaciones lineales a los grupos diferenciables descritos inicialmente en las notas de Scheffers sobre los cursos impartidos por Sophus Lie en Leipzig [S. Lie and G. Scheffers, Vorlesungen über Kontinuerliche Gruppen, Leipzig, 1893]. Veamos esta relación de manera más precisa.

El espacio tangente $T_{e} G$ al grupo de Lie $G$ en el elemento identidad $e \in G$ es isomorfo al subespacio $\mathfrak{X}_{L}(G)$ como espacio vectorial. Recordemos que $\mathfrak{X}_{L}(G)$ es el subespacio de campos vectoriales invariantes izquierdos de $\mathfrak{X}(G)$. El isomorfismo se da mediante

$$
\mathfrak{X}_{L}(G) \underset{\varphi_{2}}{\stackrel{\varphi_{1}}{\rightleftarrows}} T_{e} G
$$

donde $\varphi_{1}$ se define por $\varphi_{1}(X):=X_{e}$ para $X \in \mathfrak{X}_{L}(G)$ y $\varphi_{2}$ se define por $\varphi_{2}(\xi):=X$ con $X_{g}:=\left(L_{g}\right)^{*} \xi$ para $\xi \in T_{e} G$.

Entonces se tiene que

$$
\varphi_{1} \varphi_{2}(\xi)=\left.\varphi_{1}\left(\left(L_{g}\right)^{*} \xi\right)\right|_{g=e}=\left(L_{g}\right)^{*} \xi=\xi,
$$

además, como $X$ es invariante izquierdo, se tiene

$$
\left(\varphi_{2} \varphi_{1}(X)\right)_{g}=\left(L_{g}\right)^{*} X_{e}=X_{g} ;
$$

entonces $\varphi_{1} \varphi_{2}=i d_{T_{e} G}$ y $\varphi_{2} \varphi_{1}=i d_{\mathfrak{X}_{L}(G)}$.

Es importante notar que el subálgebra de Lie $\mathfrak{X}_{L}(G)$ es estable bajo el corchete de Lie, es decir, si $X, Y \in \mathfrak{X}_{L}(G)$, entonces $[X, Y] \in \mathfrak{X}_{L}(G)$ y por lo tanto las constantes de la estructura se preservan, de hecho tenemos

$$
\left(L_{g}\right)^{*}[X, Y]=\left[\left(L_{g}\right)^{*} X,\left(L_{g}\right)^{*} Y\right]=[X, Y] .
$$

Entonces podemos dar la siguiente :

Definición. El espacio vectorial $T_{e} G$ con la estructura de álgebra de Lie inducida por el isomorfismo con $\mathfrak{X}_{L}(G)$ es llamada el álgebra de Lie $\mathfrak{g}=$ Lie $G$ de $G$.

Veamos algunos ejemplos de álgebras de Lie asociados a grupos de Lie

- $\mathfrak{g l}_{n}(\mathbb{R}):=$ Lie $G L_{n}(\mathbb{R})=M_{n \times n}(\mathbb{R})$ 
- $\mathfrak{s l}_{n}(\mathbb{R}):=$ Lie $S L_{n}(\mathbb{R})=\left\{X \in M_{n \times n}(\mathbb{R}) \mid \operatorname{Tr}(X)=0\right\}$

- $\mathfrak{s p}_{n}(\mathbb{R}):=$ Lie $S p_{n}(\mathbb{R})=\left\{X \in M_{n \times n}(\mathbb{R}) \mid X^{T} J+J X=0\right\}$

- $\mathfrak{o}_{n}(3):=$ Lie $O(3) \cong \mathbb{R}^{3}$, con la identificación $X \leftrightarrow \xi$, y con corchete de Lie definido por $[X, Y] \leftrightarrow \xi \times \eta$ que corresponde al producto exterior en $\mathbb{R}^{3}$.

\section{A.4 Subgrupos a un parámetro y la APLICACIÓN EXPONENCIAL.}

El proceso de obtener un álgebra de Lie $\mathfrak{g}$ a partir de un grupo de Lie $G$ puede ser, en cierto sentido, localmente reversible. Daremos un tratamiento más preciso a este concepto después de definir los siguientes conceptos:

Para cada $\xi \in T_{e} G$ denotaremos por $X$ su campo vectorial invariante izquierdo asociado mediante $X_{e}=\xi$. Entonces denotamos por

$$
\begin{aligned}
\gamma_{\xi}: \mathbb{R} & \rightarrow G, \\
t & \mapsto \exp (t \xi),
\end{aligned}
$$

a la curva integral de $X$ que en el tiempo $t=0$ pasa por la identidad $e$ y cuyo vector tangente $\dot{\gamma}_{\xi}(t)$ en cada punto $\gamma_{\xi}(t)$ es igual a $X_{\gamma_{\xi}(t)}$. La existencia de la curva integral $\gamma_{\xi}(t)$ es una consecuencia del teorema de existencia y unicidad para soluciones de sistemas de ecuaciones diferenciales ordinarias. Para esta curva, se puede mostrar que

$$
\exp (t+s) \xi=\exp (t \xi) \cdot \exp (s \xi)
$$

esto nos indica que

$$
\gamma_{\xi}: \mathbb{R} \rightarrow G
$$

es un homomorfismo de grupos diferenciables. $\gamma_{\xi}$ es llamado un subgrupo a un parámetro de $G$. Por lo tanto podemos enunciar la siguiente

Definición. La aplicación

$$
\begin{aligned}
\exp : T_{e} G & \rightarrow G, \\
\xi & \mapsto \gamma_{\xi}(1)=\exp \xi,
\end{aligned}
$$

se denomina la aplicación exponencial del álgebra de Lie $\mathfrak{g}=T_{e} G$ en $G$.

Esta aplicación es diferenciable e induce la aplicación identidad sobre el espacio tangente $T_{0}\left(T_{e} G\right) \cong T_{e} G$. Sin embargo, es un difeomorfismo local pero no es un difeomorfismo sobre $G$.

Para un homomorfismo diferenciable $F: H \rightarrow G$ entre dos grupos de Lie $H$ y $G$ y para la aplicación inducida

$$
T_{e} F:=\left(F^{*}\right)_{e}: T_{e} H \rightarrow T_{e} G
$$

tenemos una regla conmutativa

$$
F\left(\exp _{H} \eta\right)=\exp _{G}\left(T_{e} F\right) \eta \quad \forall \xi \in T_{e} H=\text { Lie } H .
$$


Como la aplicación

$$
\gamma: \mathbb{R} \rightarrow F\left(\exp _{H} t \eta\right)
$$

es un subgrupo a un parámetro de $G$, es de la forma

$$
F\left(\exp _{H} \eta\right)=\gamma(1)=\gamma_{\xi}(1)=\exp _{G} \xi=\exp _{G}\left(T_{e} F\right) .
$$

Un caso especial de suma importancia es el obtenido por conjugación:

$$
\begin{aligned}
\kappa_{g}: G & \rightarrow G, \\
h & \mapsto g h g^{-1}=R_{g^{-1}} L_{g} h, \quad g \in G .
\end{aligned}
$$

Esta es una aplicación diferenciable y realmente es un automorfismo interno de $G$. Ahora denotemos por

$$
A d_{g}:=T_{e} \kappa_{g}=T_{e}\left(R_{g^{-1}} L_{g}\right): T_{e} G \rightarrow T_{e} G
$$

la aplicación adjunta asociada a $g \in G$. Como una consecuencia se sigue que

$$
\exp \left(A d_{g} \xi\right)=\kappa_{g} \exp \xi=g(\exp \xi) g^{-1},
$$

que se cumple para todo $\xi \in T_{e} G$ con $g \in G$.

Veamos algunos ejemplos de aplicaciónes exponenciales:

- $G=\mathbb{R}^{n}$, con la adición como operación de grupo, se tiene que su álgebra de Lie $\mathfrak{g}=$ Lie $G=\mathbb{R}^{n}$, y por lo tanto la aplicación exponencial será la identidad $\exp =i d_{\mathbb{R}^{n}}$

- Sea $G=G L_{n}(\mathbb{R})$ el grupo lineal con coeficientes en los reales. Para $G$ y sus subgrupos, la aplicación exponencial es la generalización de la función exponencial para matrices, por lo tanto

$$
\begin{aligned}
\exp : M_{n \times n}(\mathbb{R}) & \rightarrow G L_{n}(\mathbb{R}), \\
A & \mapsto \sum_{m=0}^{\infty} \frac{1}{m !} A^{m} .
\end{aligned}
$$

A cada $A \in M_{n \times n}(\mathbb{R})$ le pertenece un subgrupo de un parámetro $\gamma_{A}$, dado por

$$
\gamma_{A}(t)=\exp t A=\sum_{m=0}^{\infty} \frac{t^{m}}{m !} A^{m} .
$$

y de aquí podemos calcular para cada $C \in G L_{n}(\mathbb{R})$ la exponencial de una matriz conjugada como

$$
\exp \left(C A C^{-1}\right)=C(\exp A) C^{-1} .
$$

\section{A.5 DerivadA DE LiE.}

A.5.1. Derivada de Lie de una forma diferencial. Consideremos una forma diferencial $\beta \in \Omega^{p}(M ; F)$ de orden $p$ y un campo diferencial $X \in \mathfrak{X}(M)$. 
Definición A.2. La derivada de Lie de la forma $\beta$ con respecto del campo $X$ se define como

$$
\begin{array}{r}
L_{X} \beta: \mathfrak{X}(M) \times \Omega^{p}(M) \rightarrow \Omega^{p}(M), \\
L_{X} \beta=i_{X}(d \beta)+d\left(i_{X} \beta\right) .
\end{array}
$$

Evidentemente, $L_{X} \beta$ es nuevamente una forma diferencial de orden $p$. Para el caso en que $p=0$, el espacio de formas diferenciales de orden 0 son las funciones suaves $\Omega^{0}(M) \cong \mathfrak{F}(M)$ sobre la variedad. En este caso, la derivada de Lie se convierte en

$$
\begin{aligned}
L_{X} f & =i_{X}(d f)+d\left(i_{X} f\right), \\
& =i_{X}(d f) \\
& =d f(X) \\
& =X f .
\end{aligned}
$$

donde se utilizó la proposición B.27 del apéndice B. Entonces podemos establecer la siguiente

Definición A.3. Para toda $X \in \mathfrak{X}(M)$ y para $f \in \mathfrak{F}(M)$, se define la derivada de Lie de una función $f$ con respecto a un campo vectorial $X$ como

$$
\begin{aligned}
\mathfrak{X}(M) \times \mathfrak{F}(M) & \rightarrow \mathfrak{F}(M), \\
(X, f) & \mapsto L_{X} f
\end{aligned}
$$

tal que

$$
L_{X} f(p)=d f_{p}\left(X_{p}\right), \quad p \in M ;
$$

En coordenadas locales podemos escribir

$$
L_{X} f(p)=\sum_{i} a_{i}(x) \frac{\partial f}{\partial x_{i}}(x), \quad \text { si }\left.X\right|_{U}=\sum_{i} a_{i} \frac{\partial}{\partial x_{i}}(x)
$$

en la carta $(U, \varphi)$ con coordenadas $x$.

Mediante la cuarta propiedad de la proposición B.27 se puede ver inmediatamente que $L_{X}$ cumple:

$$
\begin{aligned}
L_{X}(\omega \wedge v) & =i_{X} d(\omega \wedge v)+d\left(i_{X}(\omega \wedge v)\right) \\
& =i_{X}(d \omega) \wedge v+(-1)^{p} i_{X}(\omega \wedge d v)+d\left(i_{X} \omega \wedge v\right)+(-1)^{p} d\left(\omega \wedge i_{X} v\right), \\
& =\left(L_{X} \omega\right) \wedge v+\omega \wedge\left(L_{X} v\right) .
\end{aligned}
$$

Entonces podemos ver que $L_{X}$ es un elemento de las derivaciones de $\Omega^{p}(M)$

$\mathfrak{D}\left(\Omega^{p}(M)\right)=\left\{D \in \operatorname{End}\left(\Omega^{p}(M)\right) \mid D(\alpha \wedge \beta)=\alpha \wedge D \beta+D \alpha \wedge \beta ; \alpha \wedge \beta \in \Omega^{p}(M)\right\}$.

Si $\omega$ es remplazado por $d \omega$ en A.1 uno obtiene

$$
\begin{aligned}
L_{X}(d \omega) & =i_{X} d^{2}(\omega)+d\left(i_{X}(d \omega)\right) \\
& =d\left(i_{X}(d \omega)\right) ;
\end{aligned}
$$


mientras que la diferenciación exterior de A.1 lleva a

$$
\begin{aligned}
d\left(L_{X} \omega\right) & =d\left(i_{X}(d \omega)\right)+d^{2}\left(i_{X} \omega\right) \\
& =d\left(i_{X}(d \omega)\right) ;
\end{aligned}
$$

de donde se puede ver que $L_{X}$ y $d$ conmutan

$$
d\left(L_{X} \omega\right)=L_{X}(d \omega) .
$$

A.5.2. Derivada de Lie de un Campo vectorial. También se puede definir la derivada de Lie de un campo vectorial $Y$ con respecto de otro campo vectorial $X$ imponemos la condición adicional de que $L_{X}$ sea una derivación cuando se aplica a la forma $i_{Y} \omega$, es decir

$$
L_{X}\left(i_{Y} \omega\right)=i_{\left(L_{X} Y\right)} \omega+i_{Y} L_{X} \omega .
$$

Esto determina de manera única a $L_{X} Y$. Para ver esto, supongamos que $\omega=d f$ para alguna $f \in \Omega^{0}(M)$, tenemos

$$
L_{X}\left(i_{Y}(d f)\right)=L_{X}(d f(Y))=L_{X}(Y f)=X Y f,
$$

mientras que

$$
i_{L_{X} Y} d f=d f\left(L_{X} Y\right)=\left(L_{X} Y\right) f .
$$

Utilizando la conmutatividad de $L_{X}$ y $d$ y considerando la derivada de Lie para una función, tenemos

$$
i_{Y}\left(L_{X}(d f)\right)=i_{Y} d\left(L_{X} f\right)=i_{Y} d(X f)=d(X f)(Y)=Y X f .
$$

Ahora sustituyamos los tres resultados en (A.2) con $d f=\omega$ y tenemos

$$
X Y f=\left(L_{X} Y\right) f+Y X f
$$

de donde obtenemos inmediatamente que

$$
L_{X} Y=X Y-Y X=[X, Y] .
$$

Recordemos que $\mathfrak{X}(M)$ es un álgebra de Lie con el corchete de Lie $[\cdot, \cdot]$ como producto. 



\section{APÉNDICE B}

\section{FORMAS DIFERENCIALES}

\section{B.1 Aplicaciones multilineales \\ ALTERNADAS.}

Sean $E$ y $F$ dos espacios vectoriales normados. El espacio de las aplicaciones p-lineales continuas se determina por $\mathscr{L}_{p}(E, F)$ y es el conjunto de funciones continuas lineales en cada componente

$$
\begin{aligned}
f: E \times E \times \cdots \times E & \rightarrow F . \\
\left(e_{1}, e_{2}, \cdots, e_{p}\right) & \mapsto y=f\left(e_{1}, e_{2}, \cdots, e_{p}\right)
\end{aligned}
$$

Si $p=1$ entonces se convierte en el espacio de funciones lineales continuas canónico $\mathscr{L}(E ; F)$, mientras que para $p=0$ se convierte en las funciones constantes $\mathscr{L}_{0}(E ; F)=F$

DeFinición B.1. Una aplicación $f \in \mathscr{L}_{p}(E ; F)$ se le llama alternada si su valor es nulo cada vez que se tenga $x_{i}=x_{j}$ para algún par de índices $i \neq j$, con $1 \leq i, j \leq p$.

Se conviene que toda aplicación lineal $f: E \rightarrow F$ es alternada.

Consideremos ahora que se desea hacer una multiplicación de aplicaciones multilineales alternadas, digamos $f \in \mathscr{L}_{p}(E ; F)$ y $g \in \mathscr{L}_{q}(E ; G)$, entonces necesitamos primero una aplicación bilineal continua

$$
\Phi: F \times G \rightarrow H
$$

donde $E, F, G, H$ son espacios vectoriales normados. Así podemos asociar una aplicación $h: E^{p+q} \rightarrow H$ de la forma

$$
h\left(x_{1}, \cdots, x_{p+q}\right)=\Phi\left(f\left(x_{1}, \cdots, x_{p}\right), g\left(x_{p+1}, \cdots, x_{p+q}\right) .\right.
$$


Nótese que $h \in \mathscr{A}_{p, q}(E ; H)$ no es una aplicación multilineal alternada. Definimos una aplicación que extiende las aplicaciones bilineales $h$ a aplicaciones multilineales $\tilde{h}$, a través de la aplicación lineal canónica

$$
\begin{aligned}
\varphi_{p, q}: \mathscr{A}_{p, q}(E ; F) & \rightarrow \mathscr{A}_{p+q}(E ; F) . \\
h & \mapsto \sum_{\sigma} \epsilon(\sigma)(\sigma h)
\end{aligned}
$$

donde $\sigma h$ son las permutaciones de los elementos en $h$ y $\epsilon(\sigma)$ es el signo de la permutacion.

Definición B.2. Se denomina producto exterior de $f \in \mathscr{A}_{p}(E ; F)$ y de $g \in \mathscr{A}_{q}(E ; F)$ relativo a $\Phi: F \times G \rightarrow H$ y denotado por

$$
f \wedge_{\Phi} g
$$

al elemento $\varphi_{p, q}(h) \in \mathscr{A}_{p+q}(E ; H)$, donde $h$ está definido por (B.1)

Proposición B.24. Sean $f \in \mathscr{A}_{p}(E ; \mathbb{R})$ y $g \in \mathscr{A}_{q}(E ; \mathbb{R})$ dos formas multilineales alternadas sobre los números reales, entonces

$$
g \wedge f=(-1)^{p q} f \wedge g
$$

Proposición B.25. La multiplicación exterior de formas multilineales alternadas es asociativa. Es decir, si $f \in \mathscr{A}_{p}(E ; \mathbb{R}), g \in \mathscr{A}_{q}(E ; \mathbb{R})$ y $h \in$ $\mathscr{A}_{r}(E ; \mathbb{R})$ se tiene

$$
\begin{gathered}
(f \wedge g) \wedge h=f \wedge(g \wedge h) \\
\cdots \\
\text { B.2 Formas DIFERENCIALES. }
\end{gathered}
$$

Sean $E$ y $F$ dos espacios de Banach $U \subset E$ un abierto

Definición B.3. Se denomina una forma diferencial de grado $p$, definida en $U$ y con valores en $F$ a una aplicación

$$
\omega: U \rightarrow \mathscr{A}_{p}(E ; F) .
$$

Se dice que una p-forma diferencial $\omega$ es de clase $C^{n}$ si la aplicación es de clase $C^{n}$, donde $n \in \mathbb{N} \cup\{0, \infty\}$

En esta introducción a formas diferenciales se considerará que las aplicaciones son de clase $C^{\infty}$.

Notación. $\Omega^{p}(U, F)$ representará al conjunto de todas las $p$-formas diferenciales de clase $C^{\infty}$, definidas en $U$ sobre $F$. Este conjunto tiene la estructura de un espacio vectorial.

B.2.1. Multiplicación exterior. Sean $\alpha \in \Omega^{p}(U, F)$ y $\beta \in \Omega^{q}(U, F)$. Para cada $x \in U$, tenemos los elementos $\alpha(x) \in \mathscr{A}_{p}(E, F)$ y $\beta(x) \in \mathscr{A}_{q}(E, F)$, entonces su producto exterior es

$$
\alpha(x) \wedge_{\Phi} \beta(x) \in \mathscr{A}_{p+q}(E ; H) .
$$


La aplicación

$$
\begin{aligned}
U & \rightarrow \mathscr{A}_{p+q}(E ; H) \\
x & \mapsto \alpha(x) \wedge_{\Phi} \beta(x)
\end{aligned}
$$

es de clase $C^{\infty}$ ya que es la composición de la aplicación bilineal continua

$$
x \mapsto(\alpha(x), \beta(x))
$$

que es de clase $C^{\infty}$ y de la aplicación bilineal continua

$$
\mathscr{A}_{p}(E ; H) \times \mathscr{A}_{q}(E ; H) \rightarrow \mathscr{A}_{p+q}(E ; H)
$$

definida por la multiplicación exterior.

Definición B.4. Sean $\alpha \in \Omega^{p}(U, F)$ y $\beta \in \Omega^{q}(U, F)$ Se denomina producto exterior de formas diferenciales a la aplicación

$$
\begin{aligned}
U & \rightarrow \mathscr{A}_{p+q}(E ; H) \\
x & \mapsto \alpha(x) \wedge_{\Phi} \beta(x)
\end{aligned}
$$

Esta es una aplicación bilineal definida por

$$
\Omega^{p}(U, F) \times \Omega^{q}(U, F) \rightarrow \Omega^{p+q}(U, F)
$$

Proposición B.26. La multiplicación exterior de formas diferenciales cumple las siguientes propiedades

- $\beta \wedge \alpha=(-1)^{p q} \alpha \wedge \beta$, si $\alpha$ es de grado $p$ y $\beta$ es de grado $q$.

- $(\alpha \wedge \beta) \wedge \gamma=\alpha \wedge(\beta \wedge \gamma)$

Consideremos ahora la suma directa de los espacios vectoriales $\Omega^{p}(U, \mathbb{R})$, para todos los valores de $p \geq 0$. De esta forma extendemos la multiplicación exterior por linealidad y obtenemos

$$
\Omega(U, \mathbb{R})=\bigoplus_{0 \leq p} \Omega^{p}(U, \mathbb{R})
$$

como un álgebra graduada que es anticonmutativa y asociativa.

B.2.2. Producto interior. Pasemos ahora al proceso de la multiplicación interior de las formas diferenciales. Par ello, se requiere la existencia de un campo vectorial $X \in \mathfrak{X}(E)$ de clase $C^{\infty}$ definido sobre una vecindad de una variedad $U \subset M$.

Definición B.5. Sean $\omega \in \Omega^{p}(U, F)$ una forma diferencial de orden $p$ y un campo vectorial $X \in \mathfrak{X}(M)$ definido sobre $U \subset M$. Se denomina producto interior de una forma diferencial y un campo vectorial a la aplicación

$$
\begin{aligned}
U & \rightarrow \mathscr{A}_{p-1}(M ; F) \\
x & \mapsto i_{X} \omega(x)
\end{aligned}
$$

Esta es una aplicación definida por

$$
\begin{aligned}
\Omega^{p}(U, F) \times \mathfrak{X}(U) & \rightarrow \Omega^{p-1}(U, F) \\
(\omega, X) & \mapsto i_{X} \omega
\end{aligned}
$$


También se conocen las notaciones $i(X) \omega,\langle X, \omega\rangle$ y $X\lrcorner \omega$

Proposición B.27. Sea $\omega \in \Omega^{p}(U, F)$ y $X \in \mathfrak{X}(M)$, el producto interior de la forma diferencial $\omega$ con el campo vectorial $X$ cumple las siguientes propiedades

- Para toda 0-forma $f \in \Omega^{0}(M ; F) \cong \mathfrak{F}(M)$ se tiene que

$$
i_{X} f=0
$$

- Para toda 1-forma $\alpha \in \Omega^{1}(M ; F)$ se tiene que

$$
i_{X} \omega=\omega(X)=\langle\omega, X\rangle .
$$

- Para $\omega, v \in \Omega^{p}(M ; F)$, se tiene que

$$
i_{X}(\omega+v)=i_{X} \omega+i_{X} v
$$

- Para $\omega \in \Omega^{p}(M ; F)$ y $v \in \Omega^{q}(M ; F)$, se tiene que

$$
i_{X}(\omega \wedge v)=i_{X} \omega \wedge v+(-1)^{p} \omega \wedge i_{X} v,
$$

Con las propiedades de la multiplicación interior que se presentan en la proposición anterior, tenemos como consecuencia inmedianta el siguiente resultado

Proposición B.28. Sean $X, Y \in \mathfrak{X}(M)$ y $f, g \in \Omega^{0}(M ; F) \cong \mathfrak{F}(M)$ se cumple

$$
i_{(f X+g X)} \omega=f\left(i_{X} \omega\right)+g\left(i_{Y} \omega\right)
$$

de igual forma

$$
i_{X}\left(i_{Y} \omega\right)+i_{Y}\left(i_{X} \omega\right)=0
$$

Asociado con el proceso de la multiplicación interior se encuentra el concepto de la derivada de Lie para formas diferenciales que ya se ha revisado en el Apéndice $\mathrm{A}$

B.2.3. Diferencial exterior. Sea $\omega \in \Omega^{p}(U, F)$ una forma diferencial de orden $p$, y considerando la aplicación que envia $\omega \rightarrow \mathscr{A}_{p}(E ; F)$ y su aplicación derivada $\omega^{\prime}: U \rightarrow \mathscr{L}\left(E ; \mathscr{A}_{p}(E ; F)\right)$; para todo $x \in U$ tenemos

$$
\left(\omega^{\prime}(x) \cdot \xi_{0}\right) \cdot\left(\xi_{1}, \cdots, \xi_{p}\right) \in F
$$

entonce $\omega^{\prime}(x)$ puede considerarse un elemento de $\mathscr{A}_{1, p}(E ; F)$ que podemos extender a

$$
\varphi_{1, p}: \mathscr{A}_{1, p}(E ; F) \rightarrow \mathscr{A}_{p+1}(E ; F)
$$

Definición B.6. Definimos la diferencial exterior de una forma diferencial de orden $p$, como la forma diferencial $d \omega \in \Omega^{p+1}(U, F)$ de orden $p+1$ definida por

$$
(d \omega)\left(x ; \xi_{0}, \cdots, \xi_{p}\right)=\sum_{i=0}^{p}(-1)^{i}\left(\omega^{\prime}(x) \cdot \xi_{i}\right) \cdot\left(\xi_{1}, \cdots, \hat{\xi}_{i}, \cdots, \xi_{p}\right)
$$


La diferencial exterior es una aplicación compuesta que envía

$$
U \stackrel{\omega^{\prime}}{\longrightarrow} \mathscr{A}_{1, p}(E ; F) \stackrel{\varphi_{1, p}}{\longrightarrow} \mathscr{A}_{p+1}(E ; F)
$$

Proposición B.29. Si $f \in \mathfrak{F}(E)$ es una función y $\omega \in \Omega^{p}(E, F)$ es una p-forma diferencial, se tiene

$$
d(f \cdot \omega)=(d f) \wedge \omega+f \cdot(d \omega)
$$

Proposición B.30. Si $\alpha \in \Omega^{p}(E, F)$ y $\beta \in \Omega^{q}(E, F)$ son formas diferenciales de orden $p$ y $q$ respectivamente entonces

$$
d(\alpha \wedge \beta)=(d \alpha) \wedge \beta+(-1)^{p} \alpha \wedge(d \beta)
$$

La diferencial $d(\alpha \wedge \beta) \in \Omega^{p+q+1}(E, F)$ es de orden $p+q+1$.

Teorema B.26. Sea $\omega \in \Omega^{p}(E, F)$ una forma diferencial de orden $p$, entonces se tiene que

$$
d(d \omega)=0
$$

B.2.4. Cambio de variable. Sea $U \subset E$ un abierto de un espacio de Banach $E$ y considere una forma diferencial $\omega: U \rightarrow \mathscr{A}_{p}(E ; F)$ de orden $p$. Adicionalmente consideremos una aplicación $\varphi$ entre espacios de Banach

$$
\varphi: U^{\prime} \rightarrow U
$$

con $U^{\prime} \subset E^{\prime}$. Definimos una forma diferencial de orden $p$

$$
\varphi^{*} \omega: U^{\prime} \rightarrow \mathscr{A}_{p}(E ; F)
$$

como la forma diferencial deducida de $\omega$ 19] por el cambio de variable (B.2). A esta aplicación de una forma diferencial se conoce en los libros de texto del idioma inglés como pullback, que no se conoce una traducción aceptada al español.

Lo que tendremos es que la forma $\varphi^{*} \omega$ nos proporcionará el valor de la forma diferencial en un punto $y \in U^{\prime}$ para un conjunto de vectores $\eta_{1}, \cdots, \eta_{p} \in E^{\prime}$, y este valor estará dado por

$$
\left(\varphi^{*} \omega\right)\left(y ; \eta_{1}, \cdots, \eta_{p}\right)=\omega\left(\varphi(y) ; \varphi^{\prime}(y) \cdot \eta_{1}, \cdots, \varphi^{\prime}(y) \cdot \eta_{p}\right)
$$

donde se tiene que $\varphi^{\prime}(y) \in \mathscr{L}\left(E^{\prime} ; E\right)$.

Proposición B.31. El cambio de variable $\varphi: U^{\prime} \rightarrow U$ asocia a toda forma diferencial $\omega \in \Omega^{p}(U, F)$ una forma diferencial $\varphi^{*} \omega \in \Omega^{p}\left(U^{\prime}, F\right)$. Por lo tanto

$$
\varphi^{*}: \Omega^{p}(U, F) \rightarrow \Omega^{p}\left(U^{\prime}, F\right)
$$

es una aplicación lineal.

TeORema B.27. La aplicación $\varphi^{*}$ respeta la multiplicación exterior. Sean $\alpha \in \Omega^{p}(U, F)$ y $\beta \in \Omega^{q}(U, G)$ dos formas diferenciales y sea $\Phi$ : $F \times G \rightarrow H$ una aplicación bilineal entre espacios de Banach, entonces

$$
\varphi^{*}\left(\alpha \wedge_{\Phi} \beta\right)=\left(\varphi^{*} \alpha\right) \wedge_{\Phi}\left(\varphi^{*} \beta\right) .
$$


Teorema B.28. Si $\varphi: U^{\prime} \rightarrow U$ y $f: U \rightarrow F$ son suaves entonces se tiene

$$
\varphi^{*}(d f)=d\left(\varphi^{*} f\right) .
$$

Además, si consideramos $\omega \in \Omega^{p}(U, F)$ tal que $\omega: U \rightarrow \mathscr{A}_{p}(E ; F)$ se tiene

$$
\varphi^{*}(d \omega)=d\left(\varphi^{*} \omega\right) \text {. }
$$

Proposición B.32. Sean $U \subset E, U^{\prime} \subset E^{\prime}, U^{\prime \prime} \subset E^{\prime \prime}$ abiertos de tres espacios de Banach y sean dos aplicaciones

$$
\varphi: U^{\prime} \rightarrow U, \quad \psi: U^{\prime \prime} \rightarrow U^{\prime} .
$$

Consideremos la composición $(\varphi \circ \psi): U^{\prime \prime} \rightarrow U$, entonces la aplicación

$$
(\varphi \circ \psi)^{*}: \Omega^{p}(U, F) \rightarrow \Omega^{p}\left(U^{\prime \prime}, F\right) .
$$

es equivalente a la composición

$$
(\varphi \circ \psi)^{*}=\psi^{*} \circ \varphi^{*}
$$

B.2.5. El teorema de Poincaré. Antes de enunciar el teorema de Poincaré, necesitaremos un par de definiciones

DeFinición B.7. Un subconjunto $U$ de un espacio vectorial $E$ se dice estrellado con respecto a uno de sus puntos $x_{0}$, si para cualquier $x \in U$, el segmento $\overline{x_{0} x}$, formado por los puntos $(1-t) x_{0}+t x \quad($ para $0 \leq t \leq 1)$ está contenido en $U$.

Dada la definición, podemos ver que todo conjunto estrellado es un conjunto conexo. También vemos que todo conjunto convexo es un conjunto estrellado.

Definición B.8. Una forma diferencial $\omega \in \Omega^{p}(U, F)$ de orden $p$ definida de un abierto $U \subset E$ a $F$ espacios de Banach, se dice que es cerrada si

$$
d \omega=0,
$$

Teorema B.29 (Poincaré). Sean E y F dos espacios de Banach y sea $U$ un abierto de E, estrellado con respecto a uno de sus puntos. Si $\omega \in \Omega^{p}(U, F)$ satisface

$$
d \omega=0,
$$

entonces existe una forma diferencial $\alpha \in \Omega^{p-1}(U, F)$ de grado $p-1$ tal que

$$
d \alpha=\omega .
$$

A una forma diferencial $\omega$ que cumple la propiedad (B.3) se dice que es exacta. 
El teorema de Poincaré nos indica una característica muy utilizada en los sistemas Hamiltonianos sobre variedades simplécticas. Cuando se tiene un campo vectorial simpléctico $X$, la 1-forma asociada $i_{X} \omega$ es una forma cerrada, es decir

$$
d\left(i_{X} \omega\right)=0 .
$$

Pero si además el campo vectorial es Hamiltoniano $X$, se tiene que la 1forma $i_{X} \omega$ es exacta lo que indica que existe una función $H \in \mathfrak{F}(M)$ tal que

$$
i_{X} \omega=d H \text {. }
$$

para especificar esta relación, se denota al campo vectorial $X=X_{H}$ y se dice que es un campo vectorial Hamiltoniano, con función Hamiltoniana $H: M \rightarrow \mathbb{R}$

Entonces, el teorema de Poincaré nos indica que si se tiene una forma diferencial cerrada definida sobre un abierto $U \subset E$ de un espacio de Banach, tal que ese abierto sea un conjunto convexo simplemente conexo (o que se pueda encontrar una vecindad con estas características), entonces esa forma diferencial es localmente exacta.

En términos de campos vectoriales se puede decir que todo campo vectorial simpléctico es localmente Hamiltoniano.

En realidad, el teorema de Poincaré es mucho más poderoso, para ello debemos notar que la derivada exterior de una forma diferencial nos permite crear sucesiones entre los espacios vectoriales $\Omega^{p}(M)$ para $0 \leq p \leq n$, donde $n$ es la dimensión de la variedad $M$.

B.2.6. Cohomología de De Rham. Para toda variedad $M$ de dimensión $n$ existe una sucesión exacta larga de espacios vectoriales

$$
0 \stackrel{i}{\rightarrow} \mathbb{R} \stackrel{i}{\rightarrow} \mathfrak{F}(M) \stackrel{d}{\rightarrow} \Omega^{1}(M, \mathbb{R}) \stackrel{d}{\rightarrow} \cdots \stackrel{d}{\rightarrow} \Omega^{n-1}(M, \mathbb{R}) \stackrel{d}{\rightarrow} \Omega^{n}(M, \mathbb{R}) \stackrel{d}{\rightarrow} 0
$$

generada por la derivada exterior de formas diferenciales

$$
d: \Omega^{p}(M) \rightarrow \Omega^{p+1}(M) .
$$

Entonces, dada una forma diferencial $\alpha \in \Omega^{p}(M)$ de orden $p$ y un punto $x \in$ $M$ podemos encontrar una vecindad $U \subset M$ de $x$ y una forma $\beta \in \Omega^{p-1}(M)$ de grado $(p-1)$ tal que $\alpha=d \beta$ sobre $U$.

Sin embargo, puede ser que la forma $\beta \in \Omega^{p-1}(M)$ de grado $(p-1)$ no este definida o no se pueda extender a toda la variedad $M$. Como consecuencia, para resolver $\alpha=d \beta$ localmente, una condición necesaria es que $d \alpha=0$. Sin embargo para resolver esta ecuación globalmente existen obstrucciones topológicas. Para explicarlo se requiere considerar la cohomología de De Rham.

Definición B.9. Denotemos al conjunto de las formas diferenciales cerradas de grado $k$ por $F^{k}(M)$ y por $B^{k}(M)$ a las exactas. Estos forman espacios vectoriales y en particular se tiene que $B^{k}(M) \subset F^{k}(M)$ es un 
subespacio. Para $0 \leq k \leq n$, definimos el $k$-ésimo grupo de cohomología $H^{k}(M)$ de $M$ como el cociente

$$
H^{k}(M):=F^{k}(M) / B^{k}(M) .
$$

Se puede demostrar que estos objetos son invariantes topológicos del espacio subyacente. De manera más precisa, el teorema de de Rham nos indica que estos grupos son los duales a los grupos singulares de homología del espacio. 


\section{APÉNDICE C}

\section{FUNCIONES E INTEGRALES ELÍPTICAS}

Se puede ver un tratamiento completo en [40] o [59] sobre este tópico.

\section{C.1 Integrales ELÍPticas.}

Consideremos un polinomio $P \in \mathbb{C}[z]$ de grado 3 o 4 con raíces diferentes. La diferencial holomorfa

$$
d u=\frac{d z}{\sqrt{P(z)}},
$$

se identifica de manera natural con la curva elíptica $\left\{y^{2}=P(z)\right\} \in \mathbb{C}^{2}$ mediante el isomorfismo entre el haz tangente $T \mathbb{C}$ y $\mathbb{C}^{2}$ dado por $y=\frac{d z}{d u}$. La curva elíptica $\left\{y^{2}=P(z)\right\}$ define una superficie de Riemann $\Sigma$ cuya topología es la de un toro agujerado a lo más en 4 puntos.

Sea $Q \in \mathbb{C}(x, y)$ un cociente de polinomios de dos variables con coeficientes complejos de la forma $Q(x, y)=\frac{R(x, y)}{S(x, y)}$, donde $R(x, y), S(x, y) \in \mathbb{C}[x, y]$, $S(x, y) \neq 0$ y $R(x, y), S(x, y)$ sin factor común. Entonces la integral compleja

$$
\int_{w_{0}}^{w} Q(x, y) d x, \quad w_{0}, w \in \mathbb{C},
$$

sobre la curva elíptica $\left\{y^{2}=P(x)\right\}$ es una integral elíptica general.

Los polinomios $R(x, y)$ y $S(x, y)$ restringidos a la curva elíptica $\left\{y^{2}=\right.$ $P(x)\}$ se pueden descomponer de la forma

$$
\begin{aligned}
R(x, y) & =P_{a_{1}}(x)+P_{a_{2}}(x) y \\
S(x, y) & =P_{a_{3}}(x)+P_{a_{4}}(x) y
\end{aligned}
$$


donde $P_{a_{i}} \in \mathbb{C}[x]$ son polinomios de grado $a_{i} \in \mathbb{N}, i=1, \ldots, 4$. Las expresiones (C.2) permiten escribir la integral (C.1) en términos de integrales elípticas más elementales.

DeFinición C.1. Una integral elíptica se dice que es de primera especie si $Q(x, y) d x$ induce sobre $\left\{y^{2}=P(x)\right\}$, por restricción, una 1-forma que no tiene polos. Se dice que es de segunda especie si únicamente tiene polos con residuo cero. Se dice que es de tercera especie si no es de primera o segunda especie.

De manera canónica tenemos que las integrales elípticas de primera y segunda especie pueden escribirse como

$$
\int_{w_{0}}^{w} \frac{d x}{y}, \quad \int_{w_{0}}^{w} \frac{x^{2} d x}{y}, \quad x \in \mathbb{C}, y=\sqrt{P(x)} .
$$

Nota C.15. Si el polinomio $P(x)$ tiene grado mayor que 4 y sus raíces son todas distintas entonces la curva $\left\{y^{2}=P(z)\right\}$ se conoce como una curva hipereliptica y a las integrales del tipo (C.1) se les conoce como integrales hiperelípticas generales.

C.1.1. Integrales elípticas de Jacobi. La teoría de Jacobi para las funciones e integrales elípticas se refiere a las curvas elípticas

$$
y^{2}=\left(1-x^{2}\right)\left(1-k^{2} x^{2}\right) .
$$

En ellas, las integrales elípticas de primera, segunda y tercerd 11 especie están dadas por

$$
\begin{aligned}
F(u, k) & =\int_{0}^{u} \frac{d x}{\sqrt{\left(1-x^{2}\right)\left(1-k^{2} x^{2}\right)}}, \\
E(u, k) & =\int_{0}^{u} \sqrt{\frac{1-k^{2} x^{2}}{1-x^{2}}} d x, \\
\Pi(\alpha, u, k) & =\int_{0}^{u} \frac{d x}{\left(1-\alpha x^{2}\right) \sqrt{\left(1-x^{2}\right)\left(1-k^{2} x^{2}\right)}},
\end{aligned}
$$

donde $u \in \mathbb{C}$.

Nota C.16. El nombre de integrales elípticas proviene de la integral $E(u, k)$ que corresponde a la longitud de arco de una elipse con excentricidad $e=k$. La integral $F(u, k)$ también aparece al considerar el área de un sector de una elipse de excentricidad $e=k$, de manera menos evidente. Cuando $k=0$ la elípse se convierte en una circunferencia y ambas funciones coinciden. En este caso, el área de un sector es igual a la mitad de longitud

\footnotetext{
${ }^{1}$ No existe una forma canónica para la integral elíptica de tercera especie de Jacobi. En este trabajo adoptamos esta notación que corresponde a la notación utilizada para las soluciones del problema de Sitnikov en [24] y [40. En [59] cambia la notación y se le denomina la función $\Lambda(\alpha, u, k)$, pero debemos notar que se utiliza el argumento $\alpha$ mientras que en la integral se utiliza el término $\left(1-\alpha^{2} x^{2}\right)$ con el término $\alpha^{2}$ al cuadrado.
} 
de arco sobre la circunferencia unitaria. En el texto de Markusevich [66 se da un tratamiento geométrico a estas funciones como una generalización del seno trigonométrico.

Se conoce como integral elíptica completa de primera especie, a la integral

$$
K=K(k)=\int_{0}^{1} \frac{d z}{\sqrt{\left(1-z^{2}\right)\left(1-k^{2} z^{2}\right)}}
$$

así que formalmente a la integral (C.5) se le llama la integral elíptica incompleta de primera especie. Nótese que $K=F(1, k)$ y está completamente determinada por el módulo $k$. De la misma forma se tienen las integrales elípticas completas de segunda y tercera especie dadas por

$$
E(k)=E(1, k), \quad \text { y } \quad \Pi(\alpha, k)=\Pi(\alpha, 1, k) .
$$

La integral completa de primera especie es de vital importancia en la construcción de la retícula de periodos de las funciones elípticas, como se verá más adelante.

C.1.2. Superficies de Riemann. Toda integral elíptica es multivaluada y su integrando posee cuatro puntos de ramificación que corresponden a las raíces del polinomio $P(x)$. Cuando el polinomio es de grado 3 , el cambio de carta $x \mapsto \frac{1}{u}$ permite ver que el valor $u=0$ es un punto de ramificación por lo que en la carta original, el punto de ramificación corresponde a $x=\infty$ en $\hat{\mathbb{C}}=\mathbb{C} \cup\{\infty\}$. Para obtener una función univaluada, se construye la superficie de Riemann $\Sigma$ de género 1 que corresponde a un doble recubrimiento de $\hat{\mathbb{C}}$ ramificado en las raíces de $P(x)$.

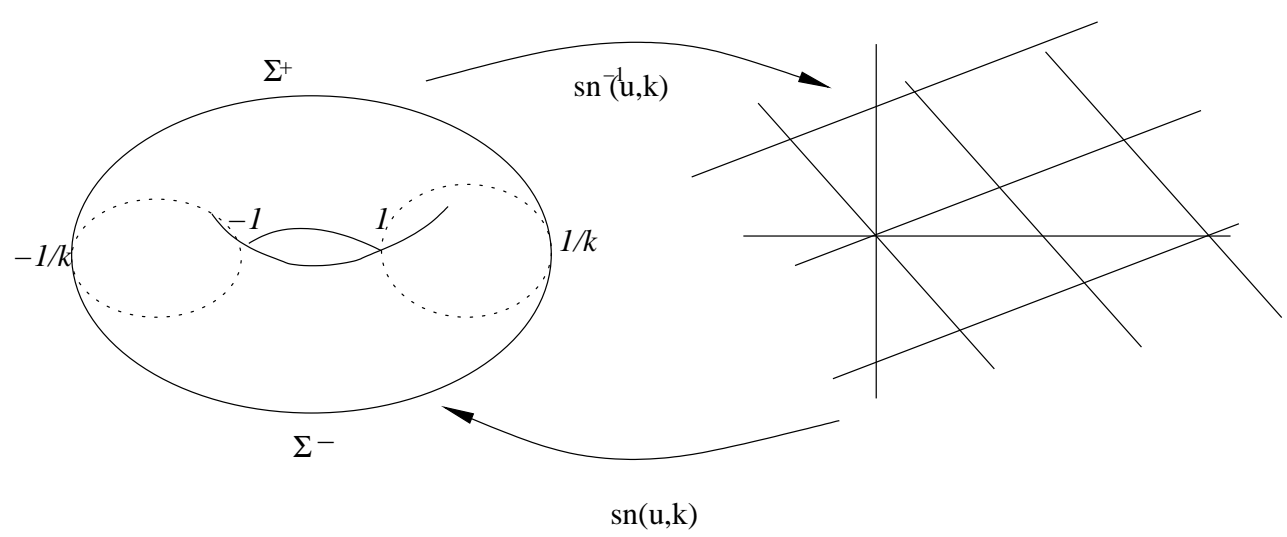

Figura C.1. La superficie de Riemann $\Sigma$ para $F(u, k)$. 


\section{C.2 Funciones elípticas.}

Partiremos de la definición de función elíptica dada en [26].

Definición C.2. Sea $\mathbb{L}=\mathbb{L}\left(\lambda_{1}, \lambda_{2}\right)$ una retícula del plano complejo de la forma

$$
\mathbb{L}=\left\{m \lambda_{1}+n \lambda_{2} \in \mathbb{C} \mid m, n \in \mathbb{Z}, \lambda_{1}, \lambda_{2} \in \mathbb{C}, \Im\left(\frac{\lambda_{1}}{\lambda_{2}}\right) \neq 0\right\} .
$$

Una función meromorfa $f: \rightarrow \mathbb{C} \cup\{\infty\}$ se llama función elíptica con respecto de $\mathbb{L}$ si para todos los $z \in \mathbb{C} y \Omega \in \mathbb{L}$, se cumple que $f(z+\Omega)=f(z)$.

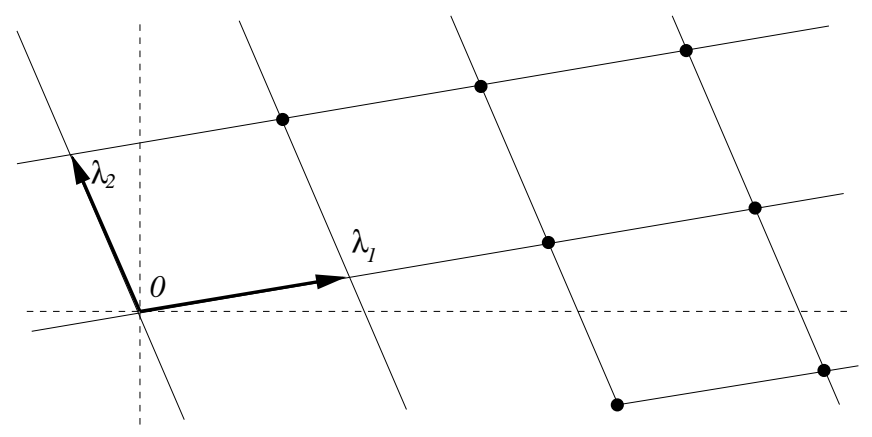

Figura C.2. La retícula $\mathbb{L}$.

En otras palabras, una función elíptica es una función meromorfa, doblemente periódica sobre el plano complejo. Esta definición se aplica a funciones elípticas generales definidas como funciones inversas de la integral elíptica (C.1) . A continuación daremos una definición alternativa de función elíptica (de primera especie).

Definición C.3. Sea $P(x)=\left(x-\alpha_{1}\right)\left(x-\alpha_{2}\right)\left(x-\alpha_{3}\right)\left(x-\alpha_{4}\right)$ un polinomio de grado 4 en $\hat{\mathbb{C}}=\mathbb{C} \cup \infty$ con todas sus raices $\alpha_{i}$ distintas. Una función elíptica de primera especie es la función inversa a la integral elíptica de primera especie

$$
\int_{x_{0}}^{x} \frac{d x}{\sqrt{P(x)}}, \quad x \in \mathbb{C} .
$$

El teorema de la inversión de Jacobi [40] nos asegura que la integral siempre está definida y por lo tanto la función inversa está bien definida. La relación entre los valores $\lambda_{1}$ y $\lambda_{2}$ de la Definición C.1 con la inversa de la integral de la Definición C.2 está dada por

$$
\lambda_{1}=2 \int_{\alpha_{i}}^{\alpha_{j}} \frac{d x}{\sqrt{P(x)}}, \quad \lambda_{2}=2 \int_{\alpha_{i}}^{\alpha_{l}} \frac{d x}{\sqrt{P(x)}},
$$


para alguna permutación $\sigma\left(\alpha_{1}, \alpha_{2}, \alpha_{3}, \alpha_{4}\right)=\left(\alpha_{i}, \alpha_{j}, \alpha_{k}, \alpha_{l}\right)$ de las raíces del polinomio $P(x)$. (Véase que el límite inferior de ambas integrales es el mismo.)

Las raíces de $P(x)$ permitieron desarrollar diferentes notaciones dadas por Jacobi, Weierstrass [59], Legendre y recientemente por Karcher [53, de donde se tienen las integrales elípticas de primera especie

$$
\begin{array}{rcl}
\text { Jacobi } & \int \frac{d x}{\sqrt{\left(1-x^{2}\right)\left(1-k^{2} x^{2}\right)}}, & k \in \mathbb{C} \backslash\{-1,0,1\}, \\
\text { Weierstrass } & \int \frac{d x}{\sqrt{4 x^{3}-g_{2} x-g_{3}}}, & \left(g_{2}^{3}-27 g_{3}^{2}\right) \in \mathbb{C} \backslash\{0\}, \\
\text { Legendre } & \int \frac{d x}{\sqrt{x(x-1)(x-\lambda)}}, & \lambda \in \mathbb{C} \backslash\{0,1, \infty\}, \\
\text { Karcher } & \int \frac{d x}{\sqrt{\left(a^{2}-x^{2}\right)\left(1-a^{2} x^{2}\right)}}, & a \in \mathbb{C} \backslash\{-1,0,1\} .
\end{array}
$$

Se puede llevar un polinomio genérico $P(x)$ de grado 4 a cualquiera de las formas canónicas de la lista anterior, mediante una translación y una transformación de Möbius. En el caso de la teoría de Jacobi (que revisaremos más adelante), se obtienen las transformaciones que llevan cualquier polinomio de grado 4 con raíces reales $a_{i} \in \mathbb{R}$ en

$$
\left(x-a_{1}\right)\left(x-a_{2}\right)\left(x-a_{3}\right)\left(x-a_{4}\right) \mapsto\left(1-x^{2}\right)\left(1-k^{2} x^{2}\right),
$$

donde $0<k<1$. Para efectos de cálculo práctico ésta es la representación más utilizada. Dichas transformaciones se pueden revisar en los capítulos 2 y 3 de $[\mathbf{5 9}$.

\section{C.3 Funciones elípticas de JaCobi.}

Se define al seno elíptico de Jacobi como la función inversa de la integral elíptica de primera especie

$$
u=\int_{0}^{\phi} \frac{d z}{\sqrt{\left(1-z^{2}\right)\left(1-k^{2} z^{2}\right)}},
$$

es decir $\operatorname{sn}(u, k)=\phi$ donde $k \in \mathbb{C} \backslash\{-1,0,1\}$ es el módulo de la función.

A partir de esta función se definen otras dos funciones elípticas, de la manera siguiente,

$$
\begin{array}{r}
\operatorname{cn}(u, k)=\sqrt{1-\operatorname{sn}^{2}(u, k)}, \\
\operatorname{dn}(u, k)=\sqrt{1-k^{2} \operatorname{sn}^{2}(u, k),}
\end{array}
$$

conocidas como el coseno elíptico de Jacobi y y la función delta de Jacobi, respectivamente.

A la función $\operatorname{sn}(u, k)$ también se le conoce como seno de amplitud 68 ya que se tiene la identidad $\operatorname{sn}(u, k)=\sin (\operatorname{am}(u, k))$, donde

$$
\operatorname{am}(u, k)=\int_{0}^{u} \operatorname{dn}(u, k) d u
$$


Inmediatamente de la definición de estas funciones podemos establecer las siguientes identidades:

$$
\begin{aligned}
c n^{2}(u, k)+s n^{2}(u, k) & =1, \\
d n^{2}(u, k)+k^{2} s n^{2}(u, k) & =1,
\end{aligned}
$$

en donde notamos su gran semejanza con las funciones trigonométricas $\sin (t)$ $\mathrm{y} \cos (t)$.

Existen varias propiedades que pueden deducirse directamente de las definiciones. De las expresiones (C.11) y (C.12) vemos que las funciones $\operatorname{cn}(u, k)$ y $\operatorname{dn}(u, k)$ son pares con respecto de $u$ mientras que $\operatorname{sn}(u, k)$ es impar. A partir de la integral (C.10), podemos ver que si el módulo $k$ de la función $\operatorname{sn}(u, k)$ es igual a cero, entonces:

$$
\int_{0}^{\phi} \frac{d z}{\sqrt{\left(1-z^{2}\right)\left(1-k^{2} z^{2}\right)}}=\int_{0}^{\phi} \frac{d z}{\sqrt{\left(1-z^{2}\right)}},
$$

de donde

$$
\begin{aligned}
\operatorname{sn}(u, 0) & =\sin (u), \\
\operatorname{cn}(u, 0) & =\cos (u), \\
\operatorname{dn}(u, 0) & =1, \\
K & =\frac{\pi}{2} .
\end{aligned}
$$

Ello que nos permite ver que las funciones elípticas de Jacobi son una generalización de las funciones trigonométricas.

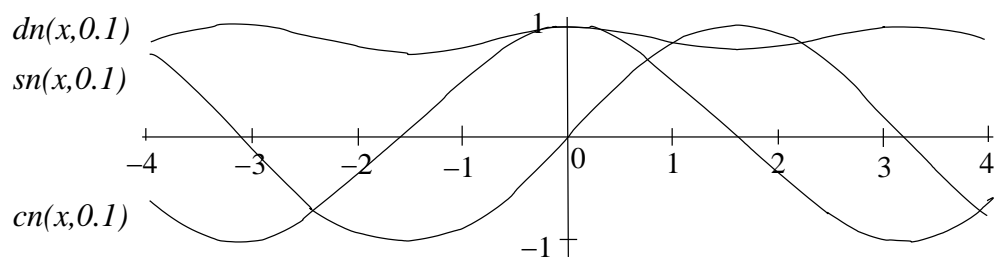

Figura C.3. Gráfica de las funciones elípticas para $k=0.1$ con argumento real.

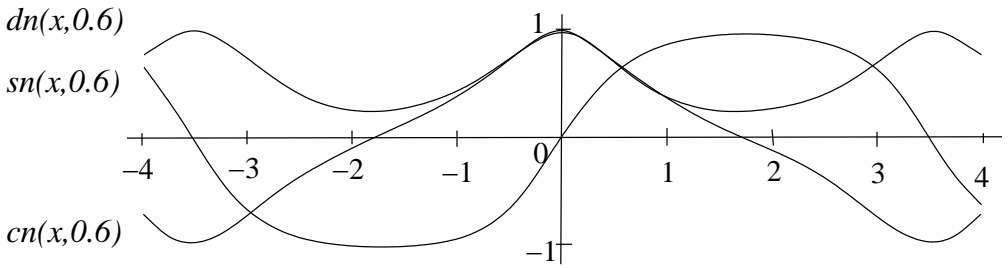

Figura C.4. Gráfica de las funciones elípticas para $k=0.6$ con argumento real. 


\section{C.4 Argumento imaginario.}

Primero se expresará a $\operatorname{sn}(i u, k)=w$ en términos de $\operatorname{sn}(u, k)$. Tomemos la función inversa

$$
i u=\int_{0}^{w} \frac{d z}{\sqrt{\left(1-z^{2}\right)\left(1-k^{2} z^{2}\right)}}
$$

y hagamos el cambio de variable $z=i \frac{\psi}{\sqrt{1-\psi^{2}}}$ para obtener

$$
i u=i \int_{0}^{-i \frac{w}{\sqrt{1+w^{2}}}} \frac{d \psi}{\sqrt{\left(1-\psi^{2}\right)\left(1-k^{2} \psi^{2}\right)}}
$$

Cancelando $i$ y aplicando la función sn( ) en ambos miembros de la ecuación anterior obtenemos

$$
\operatorname{sn}\left(u, k^{\prime}\right)=-i \frac{w}{\sqrt{1+w^{2}}},
$$

donde $k^{\prime}$ es el módulo complementario definido por $k^{\prime}=\sqrt{1-k^{2}}$. Inmediatamente se tiene la identidad $k^{\prime 2}+k^{2}=1$. Despejemos $w$ y sustituyamos $w=\operatorname{sn}(i u, k)$ para obtener la relación

$$
\operatorname{sn}(i u, k)=i \frac{\operatorname{sn}\left(u, k^{\prime}\right)}{\sqrt{1-\operatorname{sn}^{2}\left(u, k^{\prime}\right)}},
$$

que estabamos buscando. Para obtener las relaciones equivalentes para cn( ) y $\operatorname{dn}($ ) aplicamos las definiciones (C.11) y (C.12). Finalmente obtenemos

$$
\begin{aligned}
\operatorname{sn}(i u, k) & =i \frac{\operatorname{sn}\left(u, k^{\prime}\right)}{\operatorname{cn}\left(u, k^{\prime}\right)}, \\
\operatorname{cn}(i u, k) & =\frac{1}{\operatorname{cn}\left(u, k^{\prime}\right)}, \\
\operatorname{dn}(i u, k) & =\frac{\operatorname{dn}\left(u, k^{\prime}\right)}{\operatorname{cn}\left(u, k^{\prime}\right)} .
\end{aligned}
$$

Al considerar el límite del módulo complementario $k^{\prime} \rightarrow 0$, implica que $k \rightarrow 1$ y se obtienen los siguientes límites

$$
\begin{aligned}
\operatorname{sn}(u, 1) & =\frac{\sinh (u)}{\cosh (u)} \\
\operatorname{cn}(u, 1) & =\frac{1}{\cosh (u)}, \\
\operatorname{dn}(u, 1) & =\frac{1}{\cosh (u)} .
\end{aligned}
$$

Esto es muy interesante porque vemos que las funciones de Jacobi se transforman en cocientes de las funciones hiperbólicas cuando $k \rightarrow 1$. 


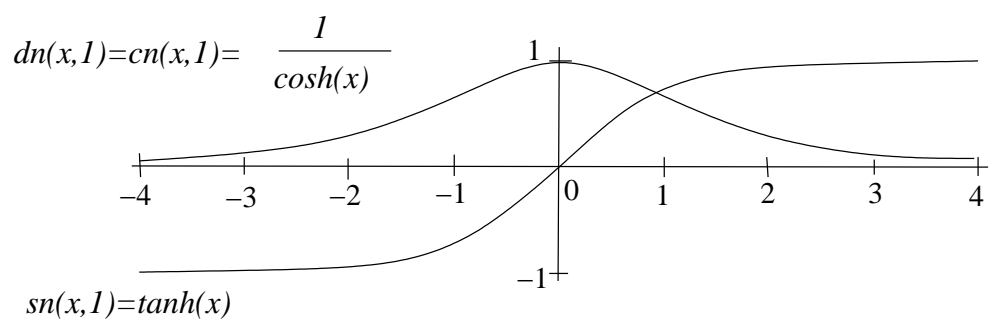

Figura C.5. Gráfica de las funciones elípticas para $k=1$, con argumento real.

\section{C.5 Derivadas De LAS FUnCIONES ELÍPTICAS DE JACOBI.}

Dada la identificación de la diferencial holomorfa

$$
d u=\frac{d z}{\sqrt{\left(1-z^{2}\right)\left(1-k^{2} z^{2}\right)}}
$$

con una curva elíptica $y^{2}=\left(1-z^{2}\right)\left(1-k^{2} z^{2}\right)$, podemos escribir

$$
\left(z^{\prime}\right)^{2}=\left(1-z^{2}\right)\left(1-k^{2} z^{2}\right)
$$

que tiene como solución $z=\operatorname{sn}(u, k)$. Reescribiendo esta ecuación tenemos

$$
\frac{d}{d u} \operatorname{sn}(u, k)=\operatorname{cn}(u, k) \operatorname{dn}(u, k) .
$$

Derivando (C.11) y (C.12) tenemos

$$
\begin{aligned}
\frac{d}{d u} \operatorname{cn}(u, k) & =-\operatorname{sn}(u, k) \operatorname{dn}(u, k), \\
\frac{d}{d u} \operatorname{dn}(u, k) & =-k^{2} \operatorname{sn}(u, k) \operatorname{cn}(u, k) .
\end{aligned}
$$

En la Sección C.7 se establece que las singularidades de los miembros derechos de las expresiones (C.21), (C.22) y C.23 son polos dobles con residuos iguales a cero (ver la sección C.7 más adelante). Esto implica que la integral de dichas expresiones no depende de la trayectoria de integración y entonces están bien definidas.

\section{C.6 TeOrema de LA ADICIÓN.}

A continuación enunciaremos el teorema de la adición para las funciones elípticas de Jacobi. Para una referencia completa de la demostración del teorema de la adición, el capítulo 4 de [68, también pueden leer la sección 2.4 de 59 . 
Teorema C.30. Sean $\alpha$ y $\beta$ dos números complejos en el dominio de $\operatorname{sn}(z, k)$, entonces se tiene que

$$
s n(\alpha+\beta)=\frac{s n(\alpha) \operatorname{cn}(\beta) d n(\beta)+\operatorname{sn}(\beta) c n(\alpha) d n(\alpha)}{1-k^{2} \operatorname{sn}^{2}(\alpha) s n^{2}(\beta)} .
$$

La idea de la demostración del teorema de la adición se basa en encontrar una integral algebraica a partir de una ecuación diferencial.

Utilizando las definiciones de $\operatorname{cn}($ ) y $\operatorname{dn}($ ), con un poco de álgebra se obtienen las relaciones

$$
\begin{aligned}
\operatorname{cn}(\alpha+\beta) & =\frac{\operatorname{cn}(\alpha) \operatorname{cn}(\beta)-\operatorname{sn}(\alpha) \operatorname{sn}(\beta) \operatorname{dn}(\alpha) \operatorname{dn}(\beta)}{1-k^{2} \operatorname{sn}^{2}(\alpha) \operatorname{sn}^{2}(\beta)}, \\
\operatorname{dn}(\alpha+\beta) & =\frac{\operatorname{dn}(\alpha) \operatorname{dn}(\beta)-k^{2} \operatorname{sn}(\alpha) \operatorname{sn}(\beta) \operatorname{cn}(\alpha) \operatorname{cn}(\beta)}{1-k^{2} \operatorname{sn}^{2}(\alpha) \operatorname{sn}^{2}(\beta)},
\end{aligned}
$$

donde hemos omitido el módulo para hacer más legible las expresiones.

\section{C.7 Periodicidad y polos.}

Para obtener los valores de los periodos de una función elíptica genérica, se deben considerar dos integrales a lo largo de los contornos simples $\gamma_{1}, \gamma_{2}$ que unen dos puntos de ramificación con un tercer punto común. Es decir, si los puntos de ramificación son $\left(\alpha_{1}, \alpha_{2}, \alpha_{3}, \alpha_{4}\right)$ entonces seleccionamos uno de ellos como un punto común, digamos $\alpha_{1}$, y consideramos los dos valores de la integral de primera especie de la siguiente forma: el primer valor a lo largo de $\gamma_{1}$ que una $\alpha_{1}$ con $\alpha_{2}$ y el segundo valor a lo largo de $\gamma_{2}$ que una $\alpha_{1}$ con $\alpha_{3}$. Específicamente tenemos los periodos determinados por

$$
\begin{aligned}
& \omega_{1}=2 \oint_{\gamma_{1}} \frac{d x}{y}=2 \int_{\alpha_{1}}^{\alpha_{2}} \frac{d x}{y}, \\
& \omega_{2}=2 \oint_{\gamma_{2}} \frac{d x}{y}=2 \int_{\alpha_{1}}^{\alpha_{3}} \frac{d x}{y} .
\end{aligned}
$$

El coeficiente 2 se requiere para considerar los contornos $\gamma_{1}$ y $\gamma_{2}$ como límites de curvas simples cerradas que encierran a los parejas de puntos de ramificación $\left(\alpha_{1}, \alpha_{2}\right)$ y $\left(\alpha_{1}, \alpha_{3}\right)$, como se ve en la Figura C.6.

Los periodos del seno elíptico de Jacobi son

$$
\begin{gathered}
4 K=4 K(k)=4 \int_{0}^{1} \frac{d z}{\sqrt{\left(1-z^{2}\right)\left(1-k^{2} z^{2}\right)}}, \\
2 i K^{\prime}=2 i K^{\prime}(k)=2 \int_{1}^{\frac{1}{k}} \frac{d z}{\sqrt{\left(1-z^{2}\right)\left(1-k^{2} z^{2}\right)}} .
\end{gathered}
$$

A continuación se muestran diversas identidades de las funciones elípticas de Jacobi que nos permiten determinar los periodos y polos de cada 


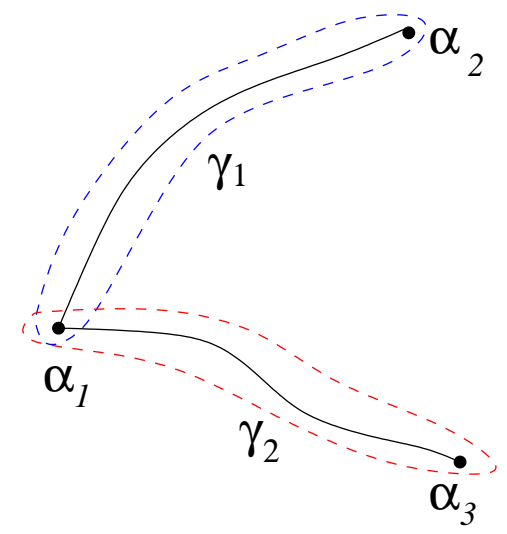

$\bullet \alpha_{4}$

Figura C.6. Periodos fundamentales para una función elíptica genérica.

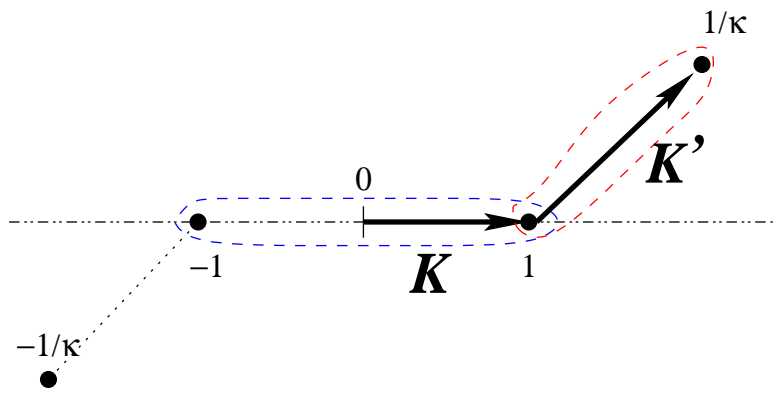

Figura C.7. Periodos fundamentales para $\operatorname{sn}(u, k)$.

una de las tres funciones. Apliquemos la función sn( ) a ambos lados de la expresión (C.29), obtendremos las relaciones

$$
\begin{aligned}
\operatorname{sn}(K, k) & =1 \\
\operatorname{cn}(K, k) & =\sqrt{1-\operatorname{sn}^{2}(K, k)}=0, \\
\operatorname{dn}(K, k) & =\sqrt{1-k^{2} \operatorname{sn}^{2}(K, k)}=\sqrt{1-k^{2}}=k^{\prime} .
\end{aligned}
$$

Utilizando el teorema de la adición con el argumento $K-t, t \in \mathbb{R}$, obtenemos

$$
\begin{aligned}
\operatorname{sn}(K-t, k) & =\frac{\operatorname{cn}(t, k)}{\operatorname{dn}(t, k)}, \\
\operatorname{cn}(K-t, k) & =k^{\prime} \frac{\operatorname{sn}(t, k)}{\operatorname{dn}(t, k)}, \\
\operatorname{dn}(K-t, k) & =\frac{k^{\prime}}{\operatorname{dn}(t, k)} .
\end{aligned}
$$


Sustituyendo $t$ por $-t$, tenemos

$$
\begin{aligned}
\operatorname{sn}(K+t, k) & =\frac{\operatorname{cn}(t, k)}{\operatorname{dn}(t, k)}=\operatorname{sn}(K-t, k), \\
\operatorname{cn}(K+t, k) & =-k^{\prime} \frac{\operatorname{sn}(t, k)}{\operatorname{dn}(t, k)}=-\operatorname{cn}(K-t, k), \\
\operatorname{dn}(K+t, k) & =\frac{k^{\prime}}{\operatorname{dn}(t, k)}=\operatorname{dn}(K-t, k) .
\end{aligned}
$$

Cambiando ahora $t$ por $K+t$, encontramos

$$
\begin{aligned}
\operatorname{sn}(t+2 K, k) & =-\operatorname{sn}(t, k), \\
\operatorname{cn}(t+2 K) & =-\operatorname{cn}(t, k), \\
\operatorname{dn}(t+2 K) & =\operatorname{dn}(t, k),
\end{aligned}
$$

de donde se deduce que uno de los periodos de $\operatorname{dn}(t, k)$ es $2 K$. Finalmente, cambiando $t$ por $t+2 K$ en las dos primeras fórmulas, obtenemos

$$
\operatorname{sn}(t+4 K, k)=\operatorname{sn}(t, k), \quad \operatorname{cn}(t+4 K, k)=\operatorname{cn}(t, k) .
$$

Una vez establecida la periodicidad en el sentido del eje real, las fórmulas (C.15), C.16) y (C.17) nos llevan a obtener las siguientes relaciones

$$
\begin{aligned}
\operatorname{sn}\left(i\left(u+2 K^{\prime}\right), k\right) & =\operatorname{sn}\left(i u, k^{\prime}\right), \\
\operatorname{cn}\left(i\left(u+2 K^{\prime}\right), k\right) & =-\operatorname{cn}\left(i u, k^{\prime}\right), \\
\operatorname{dn}\left(i\left(u+2 K^{\prime}\right), k\right) & =-\operatorname{dn}\left(i u, k^{\prime}\right),
\end{aligned}
$$

donde vemos que sn( ) tiene un periodo $i 2 K^{\prime}$. Aplicando nuevamente el argumento $i\left(u+2 K^{\prime}\right)$ a las dos últimas fórmulas, tendremos que tanto $\mathrm{cn}($ ) como $\operatorname{dn}\left(\right.$ ) tienen periodos $i 4 K^{\prime}$. Así, los periodos de las funciones de Jacobi son

$$
\begin{array}{ll}
w_{1}=4 m K+i 2 n K^{\prime}, & \operatorname{sn}(u, k), \\
w_{2}=4 m K+i 4 n K^{\prime}, & \operatorname{cn}(u, k), \\
w_{3}=2 m K+i 4 n K^{\prime}, & \operatorname{dn}(u, k),
\end{array}
$$

con $m, n \in \mathbb{Z}$.

Ahora utilicemos el teorema de la adición y las fórmulas (C.15), (C.16) y (C.17) para obtener los polos de estas funciones. Si $z=x+i y$ tendremos

$$
\begin{aligned}
\operatorname{sn}(x+i y, k) & =\frac{\operatorname{sn}(x, k) \operatorname{dn}\left(y, k^{\prime}\right)+i \operatorname{sn}\left(y, k^{\prime}\right) \operatorname{cn}(x, k) \operatorname{cn}\left(y, k^{\prime}\right) \operatorname{dn}(x, k)}{\operatorname{cn}^{2}\left(y, k^{\prime}\right)+k^{2} \operatorname{sn}^{2}(x, k) \operatorname{sn}^{2}\left(y, k^{\prime}\right)} \\
\operatorname{cn}(x+i y, k) & =\frac{\operatorname{cn}(x, k) \operatorname{cn}\left(y, k^{\prime}\right)-i \operatorname{sn}(x, k) \operatorname{sn}\left(y, k^{\prime}\right) \operatorname{dn}(x, k) \operatorname{dn}\left(y, k^{\prime}\right)}{\operatorname{cn}^{2}\left(y, k^{\prime}\right)+k^{2} \operatorname{sn}^{2}(x, k) \operatorname{sn}^{2}\left(y, k^{\prime}\right)} \\
\operatorname{dn}(x+i y, k) & =\frac{\operatorname{dn}(x, k) \operatorname{cn}\left(y, k^{\prime}\right) \operatorname{dn}\left(y, k^{\prime}\right)-i k^{2} \operatorname{sn}(x, k) \operatorname{cn}(x, k) \operatorname{sn}\left(y, k^{\prime}\right)}{\operatorname{cn}^{2}\left(x, k^{\prime}\right)+k^{2} \operatorname{sn}^{2}(x, k) \operatorname{sn}^{2}\left(y, k^{\prime}\right)}
\end{aligned}
$$

debido a que las funciones $\operatorname{sn}(), \operatorname{cn}()$ y $\operatorname{dn}($ ), definidas sobre el plano complejo de la manera anterior, son racionales, entonces estas funciones 
tienen sus polos en aquellos valores de $z=x+i y$ que anulen al denominador. Esto nos lleva a la relación

$$
\operatorname{cn}^{2}\left(y, k^{\prime}\right)+k^{2} \operatorname{sn}^{2}(x, k) \operatorname{sn}^{2}\left(y, k^{\prime}\right)=0,
$$

donde $0<k, k^{\prime}<1$.

Ya que $x, y$ son reales, la relación anterior se cumple si se dan simultáneamente las condiciones

$$
\operatorname{cn}\left(y, k^{\prime}\right)=0 \quad \text { y } \quad \operatorname{sn}(x, k) \operatorname{sn}\left(y, k^{\prime}\right)=0 .
$$

Si $\operatorname{cn}\left(y, k^{\prime}\right)=0$, entonces $\operatorname{sn}\left(y, k^{\prime}\right)= \pm 1$, por lo tanto $\operatorname{sn}(x, k)$ debe ser cero. De aqui se deduce que

$$
x=2 m K \quad \text { y } \quad y=(2 n-1) K^{\prime},
$$

que podemos escribir como

$$
z=2 m K+i(2 n-1) K^{\prime}, \quad m, n \in \mathbb{Z} .
$$

Estos valores de $z$ representan todos los polos posibles de las funciones elipticas de Jacobi.

Para establecer cuales de esos polos pertenecen a la misma clase de equivalencia, necesitamos un concepto adicional.

Definición C.4. Consideremos la retícula $\mathbb{L}=\mathbb{L}\left(\lambda_{1}, \lambda_{2}\right)$ asociada a una función elíptica $f$. Un dominio fundamental de la función elíptica $f$ con respecto de la retícula $\mathbb{L}$ basado en $z_{0}$, es el conjunto

$$
D_{f}\left(z_{0}\right)=\left\{z_{0}+\mu \lambda_{1}+\delta \lambda_{2} \in \mathbb{C} \mid 0 \leq \mu, \delta<1\right\} .
$$

Las singularidades de las tres funciones elípticas se encuentran en $u=$ $2 m K+i(2 n-1) K^{\prime}$. Consideremos el dominio fundamental $D_{\mathrm{sn}}(0)$ de la función $\operatorname{sn}(u, k)$, donde podemos ver que existen dos singularidades: una en $u=i K^{\prime}$ y la otra en $u=2 K+i K^{\prime}$.

Para conocer el valor de los residuos de estos polos debemos obtener el desarrollo en serie de cada una de las funciones elípticas en una vecindad de $u=0$. El desarrollo de cada una de las tres funciones es

$$
\begin{aligned}
& \operatorname{sn}(u, k)=u-\frac{1}{3 !}\left(1+k^{2}\right) u^{3}+\frac{1}{5 !}\left(1+14 k^{2}+k^{4}\right) u^{5}-\ldots \\
& \operatorname{cn}(u, k)=1-\frac{1}{2 !} u^{2}+\frac{1}{4 !}\left(1+4 k^{2}\right) u^{4}-\ldots \\
& \operatorname{dn}(u, k)=1-\frac{1}{2 !} k^{2} u^{2}+\frac{1}{4 !}\left(4 k^{2}+k^{4}\right) u^{4}-\ldots
\end{aligned}
$$

Aplicando el teorema de la adición con argumento $z=x+i y$ obtenemos

$$
\operatorname{sn}\left(i K^{\prime}+u\right)=\frac{1}{k \operatorname{sn}(u, k)} .
$$

Mediante el desarrollo en serie en una vecindad de $u=0$ tenemos

$$
\operatorname{sn}\left(i K^{\prime}+u\right)=\frac{1}{k u}+\frac{1}{6 k}\left(1+k^{2}\right) u+\ldots,
$$


de donde vemos que $\operatorname{sn}()$ tiene un polo simple en $u=i K^{\prime}$ con residuo $1 / k$. La otra singularidad que existe en el dominio fundamental $D_{\mathrm{sn}}(0)$ es $u=2 K+i K^{\prime}$. Calculando tenemos que

$$
\operatorname{sn}\left(2 K+i K^{\prime}+u\right)=-\operatorname{sn}\left(i K^{\prime}+u\right)=-\frac{1}{k u}+\ldots,
$$

por lo tanto $\mathrm{sn}(\mathrm{)})$ tiene un polo simple en $u=2 K+i K^{\prime}$ con residuo $-1 / k$.

Aplicando el mismo procedimiento para $\mathrm{cn}(\mathrm{)}$ y $\mathrm{dn}(\mathrm{)}$ ), obtendremos los residuos de las singularidades correspondientes. Sin embargo, sabemos que los dominios fundamentales de ambas funciones son diferentes (tomados a partir de las fórmulas (C.43), (C.44) y (C.45)). En un dominio fundamental $D_{\text {cn }}(0)$ tenemos las singularidades $u=i K^{\prime}$ y $u=2 K+i K^{\prime}$, mientras que en un dominio $D_{\mathrm{dn}}(0)$, tenemos las singularidades $u=i K^{\prime}$ y $u=3 i K^{\prime}$. Calculemos cuales son los residuos de cada una de las singularidades anteriores.

Para cn( ) tenemos que

$$
\begin{aligned}
\operatorname{cn}\left(i K^{\prime}+u\right) & =\frac{\operatorname{dn}(u, k)}{i k \operatorname{sn}(u, k)}, \\
\operatorname{cn}\left(2 K+i K^{\prime}+u\right) & =-\operatorname{cn}\left(i K^{\prime}+u\right)
\end{aligned}
$$

y utilizando el desarrollo en serie alrededor de $u=0$ tenemos

$$
\begin{aligned}
\operatorname{cn}\left(i K^{\prime}+u\right) & =\frac{1}{i k u}+\frac{1}{6 i k}\left(1-2 k^{2}\right) u+\ldots, \\
\operatorname{cn}\left(2 K+i K^{\prime}+u\right) & =-\frac{1}{i k u}-\frac{1}{6 i k}\left(1-2 k^{2}\right) u+\ldots .
\end{aligned}
$$

Las relaciones anteriores muestran que $\mathrm{cn}($ ) tiene un polo simple con residuo $-i / k$ en $u=i K^{\prime}$, mientras que $u=2 K+i K^{\prime}$ es otro polo simple con residuo $i / k$.

Finalmente, para dn( ) tenemos que

$$
\begin{aligned}
\operatorname{dn}\left(i K^{\prime}+u\right) & =\frac{\operatorname{cn}(u, k)}{i \operatorname{sn}(u, k)}, \\
\operatorname{dn}\left(3 i K^{\prime}+u\right) & =-\operatorname{dn}\left(i K^{\prime}\right) .
\end{aligned}
$$

Utilizando el desarrollo en serie alrededor de $u=0$ tenemos

$$
\begin{aligned}
\operatorname{dn}\left(i K^{\prime}+u\right) & =\frac{1}{i u}-\frac{1}{6 i}\left(2-k^{2}\right) u+\ldots, \\
\operatorname{dn}\left(3 i K^{\prime}+u\right) & =-\frac{1}{i u}+\frac{1}{6 i}\left(2-k^{2}\right) u+\ldots,
\end{aligned}
$$

de donde podemos ver que $\operatorname{dn}()$ tiene dos polos simples en $u=i K^{\prime} \mathrm{y}$ $u=3 i K^{\prime}$ con residuos $-i$ e $i$ respectivamente.

Utilizando un poco de álgebra podemos ver que el producto de cualesquiera dos de estas funciones produce polos dobles en cada singularidad (por la fórmula (C.46), las tres funciones comparten el mismo conjunto de 
singularidades), con residuo cero. Por lo tanto, las integrales

$$
\begin{aligned}
\int \operatorname{cn}(u, k) \operatorname{dn}(u, k) d u & =\operatorname{sn}(u, k), \\
\int-\operatorname{sn}(u, k) \operatorname{dn}(u, k) d u & =\operatorname{cn}(u, k), \\
\int-k^{2} \operatorname{sn}(u, k) \operatorname{cn}(u, k) d u & =\operatorname{dn}(u, k)
\end{aligned}
$$

están bien definidas y son univaluadas, así que $\int \operatorname{sn}^{\prime}(u, k)=\operatorname{sn}(u, k)$ está bien definida. Esto mismo sucede con las integrales de los cuadrados de las funciones de Jacobi

$$
\begin{aligned}
& \int \operatorname{sn}^{2}(u, k) d u, \\
& \int \operatorname{cn}^{2}(u, k) d u, \\
& \int \operatorname{dn}^{2}(u, k) d u
\end{aligned}
$$

En particular, la última integral se conoce como la función $E$ de Jacobi:

$$
E(u, k)=\int_{0}^{u} \operatorname{dn}^{2}(v, k) d v
$$

que es una integral elíptica de segunda especie.

\section{C.8 Transformaciones Del módulo.}

Existen dos transformaciones básicas del módulo $k$. La primera se obtuvo al considerar el argumento imaginario y enviaba $k \mapsto \sqrt{1-k^{2}}$. La otra transformación básica corresponde a enviar $k \mapsto \frac{1}{k}$.

En esta sección veremos como se transforman las funciones de Jacobi bajo la transformación del módulo $k \mapsto \frac{1}{k}$. El método es realizar un cambio de variable de la forma

$$
\phi=k z .
$$

Sea $\operatorname{sn}(w, k)=\varphi$, entonces:

$$
w=\int_{0}^{\varphi} \frac{d z}{\sqrt{\left(1-z^{2}\right)\left(1-k^{2} z^{2}\right)}} .
$$

Aplicando el cambio de variable (C.69) en la fórmula (C.70), tenemos $z=\frac{1}{k} \phi$ y $d z=\frac{1}{k} d \phi$, de aquí:

$$
\begin{aligned}
w & =\frac{1}{k} \int_{0}^{k \varphi} \frac{d \phi}{\sqrt{\left(1-\frac{1}{k^{2}} \phi^{2}\right)\left(1-\phi^{2}\right)}} \\
k w & =\int_{0}^{k \varphi} \frac{d \phi}{\sqrt{\left(1-\frac{1}{k^{2}} \phi^{2}\right)\left(1-\phi^{2}\right)}}
\end{aligned}
$$


con lo cual el nuevo módulo de la función es $\frac{1}{k}$. Aplicando la función sn( ) a ambos lados de la igualdad anterior tenemos:

$$
\begin{aligned}
\mathrm{sn}\left(k w, \frac{1}{k}\right) & =k \varphi, \\
\frac{1}{k} \mathrm{sn}\left(k w, \frac{1}{k}\right) & =\varphi .
\end{aligned}
$$

Finalmente, sabemos que $\frac{1}{k} \operatorname{sn}\left(k w, \frac{1}{k}\right)=\varphi=\operatorname{sn}(w, k)$, por lo que las funciones de Jacobi quedarán de la siguiente manera:

$$
\begin{aligned}
\operatorname{sn}(w, k) & =\frac{1}{k} \operatorname{sn}\left(k w, \frac{1}{k}\right), \\
\operatorname{cn}(w, k) & =\operatorname{dn}\left(k w, \frac{1}{k}\right), \\
\operatorname{dn}(w, k) & =\operatorname{cn}\left(k w, \frac{1}{k}\right) .
\end{aligned}
$$

Nota C.17. El módulo $k$ de las funciones elípticas de Jacobi, no es una función modular en el sentido moderno de este término. Por el contrario, $k^{2}=\lambda(\tau)$ donde $\tau=i \frac{K}{K^{\prime}}$ si es una función modular y las transformaciones descritas en esta sección corresponden a los generadores del grupo anharmónico $\mathfrak{H}$. Estos generadores se escriben como

$$
\lambda \mapsto 1-\lambda, \quad \mathrm{y} \quad \lambda \mapsto \frac{1}{\lambda},
$$

por lo tanto, los campos de funciones meromorfas $\mathcal{M}(T)$ sobre el toro complejo $T=\mathbb{C} / \mathbb{L}$ que son isomorfos a $\mathcal{M}(T) \cong \mathbb{C}\left(s n, s n^{\prime}\right)$ son conformemente equivalentes si y sólo si $k^{2} \in \mathfrak{H}$. En la teoría de Weierstrass sólo existe un representante $\wp$ para cada toro complejo $T$ tal que $\mathcal{M}(T) \cong \mathbb{C}\left(\wp, \wp^{\prime}\right)$.

Existen varias transformaciones adicionales para el módulo, que pueden generalizarse considerando transformaciones de tipo

$$
k_{1}=\frac{c+d k}{a+b k}
$$

donde $a, b, c, d \in \mathbb{Z}$ tal que $0<(a d-c b)$. Sin embargo, las funciones elípticas obtenidas ya no son conformemente equivalentes. Para una discusión más extensa acerca de las transformaciones modulares, el lector puede revisar el capítulo 9 de [59]. 



\section{BIBLIOGRAFÍA}

[1] T. Abraham and J. Marsden, Foundations of Mechanics, Second Edition, AddisonWesley, 1987.

[2] V. M. Alekseev, Quasirandom Dynamical Systems I, II, III, Math USSR Sbornik, 5, pp 73-128; 6, pp 505-560; 7, 1-43, 1968.

[3] M. Alvarez and J. Llibre, Heteroclinic Orbits and Bernoulli Shift for the Elliptic Collision Restricted Three-Body Problem, Arch Rational Mech Anal. 156, 2001, pp 317-357.

[4] V. I. Arnold, Mathematical Methods of Classical Mechanics, Springer-Verlag, 1978.

[5] M. Audin, A. Cannas da Silva and E. Lerman Simplectic Geometry of Integrable Hamiltonian Systems, Advanced Courses in Mathematics CRM, Birkhäuser, 2003.

[6] M. Audin, Les systémes hamiltoniens et leur intégrabilité, Cours Spécialisés 8, Société Mathématique de France, EDP Sciences, 2001.

[7] T. Bartsch, The Kustaanheimo-Stiefel transformation in geometric algebra, arXiv:physics/0301017v1, 10Jan2003.

[8] E. Belbruno, J. Llibre and M. Ollé, On the Families of Periodic Orbits wich Bifurcate from the Circular Sitnikov Motions, Cel. Mech. and Dyn. Astr. 96, 1994, pp 99-129.

[9] R. Berndt, An Introduction to Symplectic Geometry, Graduate Studies in Mathematics 26, AMS, 2000.

[10] D. Boccaletti and G. Pucacco, Theory of Orbits 1: Integrable Systems and Nonperturbative Methods, Astronomy and Astrophysics Library, Springer Verlag, 2001.

[11] S. V. Bolotin, Second Species Periodic Orbits of the Elliptic 3 Body Problem, Cel. Mech. and Dyn. Astr. 93, 2005, pp 343-371.

[12] S. V. Bolotin and R. S. MacKay, Periodic and chaotic trajectories of the second species for the n-center problem, Cel. Mech. and Dyn. Astr. 77, 2000, pp 49-75.

[13] D. Brouwer and Clemence, Methods of Celestial Mechanics, Academic Press, 1961.

[14] R. L. Bryant, An Introduction to Lie Groups and Symplectic Geometry, Electronic version, Lectures at the Regional Geometry Institute in Park City 1991, Durham NC, last modified 2003.

[15] A. Cannas da Silva, Introduction to Symplectic and Hamiltonian Geometry, Publicações Matemáticas, PM-11,IMPA, 1996.

[16] A. Cannas da Silva, Lectures on Symplectic Geometry, Springer-Verlag, 2000.

[17] C. Caramanis, Notes on Ergodic Theory, disponible en formato electrónico en http://web.mit.edu/ cmcaram/www/pubs/ergodic_theory.pdf 1999

[18] A. Celletti, Singularities, Collisions and Regularization Theory, Lectures Notes in Physics 590, Benest and C. Froeschlé (eds), Singularities in Gravitational Systems, Springer-Verlag, pp 1-24.

[19] H. Cartan, Formas Diferenciales, Editorial Omega, 1972. 
[20] J. Chazy, Sur l'allure du mouvement dans le problème des trois corps quand le temps crô̂t indéfiniment. Annales scientifiques de l'École Normale Supérieure Sér. 3, 39, 1922, pp 29-130.

[21] S. R. Chesley, A Global Analysis of the Generalized Sitnikov Problem, Cel. Mech. and Dyn. Astr. 73, 1999, pp 291-302.

[22] M. S. Child, Molecular Collison Theory, Dover, 1974.

[23] M. Coornaert and A. Papadopoulos, Symbolic Dynamics and Hyperbolic Groups, Lecture Notes in Mathematics 1539, Springer-Verlag, 1993.

[24] M. Corbera, J. Llibre and P. Torres, Global existence of subharmonic orbits in the Sitnikov problem, Versión preliminar, 2004.

[25] M. Corbera and J. Llibre, On Symmetric Periodic Orbits of the Elliptic Sitnikov Problem Via the Analytic Continuation Method, Contemporary Mathematics, 292, pp 91-127, AMS, 2002.

[26] da Costa, Celso José, Funções Elípticas, Algébricas e Superfícies Mínimas 18o. Colóquio Brasileiro de Matemática, IMPA, 1991.

[27] H. Dankowicz and P. Holmes, The Existence of Transverse Homoclinic Points in the Sitnikov Problem, Journal of Differential Equations 116, 1995, pp 468-483.

[28] A. Deprit, Elimination of the nodes in problems of $n$ bodies, Cel. Mech. and Dyn. Astr. 30, 1983 pp 181-195.

[29] M. P. do Carmo, Geometria Riemanniana, Terceira Edição, Projeto Euclides, IMPA, 2005.

[30] R. Dvorak and Yi Sui Sun, The phase space structure of the extended Sitnikov problem, Cel. Mech. and Dyn. Astr. 67, 1997, pp 87-106.

[31] R. Dvorak, Numerical results to the Sitnikov problem, Cel. Mech. and Dyn. Astr. 56, 1993, pp 71-80.

[32] E. Fermi, On the Origin of the Cosmic Radiation, Physical Review 75, No. 8, 1949, pp 1169-1174.

[33] A. García and E. Pérez-Chavela, Heteroclinic Phenomena in the Sitnikov Problem, Hamiltonian Systems and Celestial Mechanics (HAMSYS-98), World Scientific, 2000, pp 174-185.

[34] V. Gorvatsevich, A. Onishchik and E. Vinberg, Foundations of Lie Theory and Lie Transformation Groups, Springer-Verlag, 1997.

[35] J. Guckenheimer and P. Holmes, Nonlinear Oscillations, Dynamical Systems, and Bifurcations of Vector Fields, Springer-Verlag, 1983.

[36] V. Guillemin and S. Stengberg Convexity Properties of the Moment Mapping, Invent. Math. 69, 1982 pp 491-513.

[37] V. Guillemin and S. Stengberg Variations on a Theme by Kepler, Colloquium Publications 42, AMS, 1990.

[38] G. Haller, Chaos Near Resonances, Applied Mathematical Science 138, SpringerVerlag, 1999.

[39] Y. Hagihara, Celestial Mechanics, Vol IV, Part 1, Japan Society for the Promotion of Science, 1975.

[40] H. Hancock, Lectures on the Theory of Elliptic Functions, John Wiley \& Sons, 1910.

[41] D. Hestenes, New foundations for classical mechanics, Fundamental Theories of Physics, Kluwer, 1990.

[42] D. Hestenes and G. Sobczyk, Clifford Algebra to Geometric Calculus, Fundamental Theories of Physics, Reidel, 1984.

[43] H. Hofer and E. Zender, Symplectic Invariants and Hamiltonian Dynamics, Birkhäuser, 1994.

[44] F. Iachello, Lie Algebras and Applications, Lecture Notes in Physics 708, SpringerVerlag, 2006.

[45] A. Ibort and J. Marín-Solano Coisotropic regularization of singular Lagrangians, ESI Institute for Mathematical Physics, Preprint ESI 236, 1995. 
[46] M. A. Jalali and S. H. Pourtakdoust, Regular and Chaotic Solutions of the Sitnikov Problem Near the 3/2 Commensurability, Cel. Mech. and Dyn. Astr. 68, 1997, pp 151-162.

[47] L. Jiménez-Lara and A. Escalona Buendía, Symmetries and bifurcations in the Sitnikov problem, Cel. Mech. and Dyn. Astr. 79, 2001, pp 97-117.

[48] H. Jiménez-Pérez and E. Lacomba Symplectic Regularization in Celestial Mechanics, preprint 2009.

[49] H. Jiménez-Pérez and E. Lacomba On the Periodic Orbits of the Double Sitnikov Problem, C. R. Acad. Sci. Paris, Ser. I, 2009.

[50] H. Jiménez-Pérez and E. Lacomba Energy Levels of Periodic Solutions of the Circular 2+2 Sitnikov Problem, Qual. Theo. Dyn. Sys. 2009.

[51] H. Jiménez-Pérez and L. Franco Symplectic Regularization On $T^{*} \mathbb{R}^{3}$, (en preparación).

[52] H. Jiménez-Pérez, Non-meromorphic integrability of the 2+2 Sitnikov problem, preprint 2008.

[53] H. Karcher, Elliptic Functions with Simple Symmetries and Fast Addition Formulas, Internet draft.

[54] B. P. Kitchens, Symbolic Dynamics, One-sided, Two-side and Countable State Markov Shifts, Springer-Verlag, 1997.

[55] T. Kovács and B. Érdi, The structure of the extended phase space of the Sitnikov problem, Astron. Nachr. 328, No.8, 2007, pp 801-804.

[56] E. Lacomba y H. Jiménez Notas de curso de Sistemas Hamiltonianos.

[57] E. Lacomba, J. Libre and E. Pérez-Chavela, The Generalized Sitnikov Problem, Contemporary Mathematics 292, AMS, 2002, pp 147-158

[58] E. Lacomba, Infinity Manifolds for Positive Energy in Celestial Mechanics, Contemporary Mathematics 58, Part III, AMS, 1987, pp 193-201.

[59] D. F. Lawden, Elliptic Functions and Applications, Applied Mathematical Sciences 98, Springer-Verlag, 1989.

[60] J. M. Lee, Differential Geometry, Analisys and Physics, Internet draft, 2000.

[61] A. J. Lichtenberg and M. A. Lieberman, Regular and Chaotic Dynamics, Second Edition, Applied Mathematical Science 38, Springer-Verlag, 1992.

[62] Jie Liu and Yi Sui Sun, On the Sitnikov problem, Cel. Mech. and Dyn. Astr. 49, 1990, pp 285-302.

[63] J. Llibre and R Ortega, On the families of periodic orbits of the Sitnikov problem, SIAM J. Appl. Dyn. Sys. 7, No. 2, 2008, pp. 561576.

[64] D. Lovelock and H. Rund, Tensors, Differential Forms, and Variational Principles, Dover, 1975.

[65] W. D. MacMillan, An Integrable Case in the Restricted Problem of Three Bodies, Astronomical Journal 27, 1911, pp 11-13.

[66] C. Marchal, Regularization of the Singularities of the N-Body Problem, NATO Advanced Study Institute Series, Applications of Modern Dynamics to Celestial Mechanics and Astrodynamics, Serie C, Mathematical and Physical Sciences 82, 1981, pp 201-236.

[67] J. P. Marco and L. Niederman Sur la construction des solutions de seconde espèce dans le problème plan restreint des trois corps, Annales de l'I.H.P. Section A, Tome 62, No. 3, 1995, pp 211-249.

[68] A. I. Markushevich, The Remarkable Sine Functions, Elsevier, 1966.

[69] D. McDuff y D. Salomon, Introduction to Symplectic Topology, 2nd Edition, Oxford Mathematical Monographs, Oxford University Press, 1998.

[70] R. McGehee, A Stable Manifold Theorem for Degenerate Fixed Points with Applications to Celestial Mechanics, J. Differential Equations 14, 1973, pp 70-88.

[71] R. McGehee, Triple Collisions in Newtonian Gravitational Systems, Dynamical Systems, Theory and Applications, J. Moser, ed., 1975, pp 550-572. 
[72] R. McGehee, Von Zeipel's theorem on singularities in celestial mechanics, Expo. Math. 4, 1986, pp 335-345.

[73] H. McKean and V. Moll, Elliptic Curves, Cambridge University Press, 1999.

[74] K. R. Meyer and G. R. Hall, An Introduction to Hamiltonian Dynamical Systems, Springer-Verlag, 1991.

[75] K. R. Meyer, Periodic Solutions of the N-Body Problem, Lecture Notes in Mathematics 1719, Springer-Verlag, 1999.

[76] A. Milani and A. M. Nobili, Instability of the 2+2 Body Problem, Cel. Mech. and Dyn. Astr. 41, 1988, pp 153-160.

[77] J. Moser, Stable and Random Motions in Dynamical Systems, Annals of Math Studies 77, Princeton Univ. Press, 1973.

[78] J. Moser, Hidden Symmetries in Dinamical Systems, American Scientist, 1979, pp 689-695.

[79] J. Moser, Various Aspects of Integrable Hamiltonian Systems, Dynamical Systems, CIME Lectures, Birkhäuser, 1980, pp 233-290.

[80] F. R. Moulton, An Introduction to Celestial Mechanics, Second edition, Dover, 1970.

[81] P. Painlevé, Leçons sur la Théorie Analitique des Equations Differentielles Professées à Stockholm, A. Hermann, 1897.

[82] G. Pavanini, Sopra una nuova categoria di soluzioni periodiche nel problema dei tre corpi, Annali di Mathematica, Serie III, Tomo XIII, 1907, pp 179-202.

[83] A. M. Perelomov, Integrable Systems of Classical Mechanics and Lie Algebras, Birkhäuser, 1990.

[84] E. Perdios and V. V. Markellos, Stability and bifurcations of Sitnikov motions, Cel. Mech. and Dyn. Astr. 42, 1988, pp 187-200.

[85] H. Pollard, Celestial Mechanics, Mathematical Association of America, 1976.

[86] C. Pugh and C. Robinson, The C-closing lemma, including Hamiltonians, Ergodic Theory and Dynamical Systems 3, 1983 pp 261-314.

[87] M. Puta, Hamiltonian mechanical systems and geometrical quantization, Kluwer, 1993.

[88] J. Rawnsley Symplectic Geometry and Momentum Maps, Master Course Notes, Mathematics Institute, University of Warwick, 2000.

[89] N. Roy, Intersections of Lagrangian submanifolds and the Melnikov 1-form, versión preliminar arxiv:math-ph/0505067v2, 2005.

[90] T. Schneider, Einführung in die Transzendenten Zahlen, Springer-Verlag, 1957.

[91] P. Shields, The Theory of Bernoulli Shifts, Chicago Lectures in Mathematics, The University of Chicago Press, 1973.

[92] C. Siegel and J. Moser Lectures on Celestial Mechanics, Classics in Mathematics, Springer-Verlag reprint, 1995.

[93] C. Simó, Masses for which Triple Collision is Regularizable, Cel. Mech. and Dyn. Astr. 21, 1980, pp 25-36.

[94] K. Sitnikov, Existence of oscillating motions for the three-body problem, Dokl. Akad. Nauk. 133, No. 2, 1960, pp 303-306.

[95] P. S. Soulis, K. E. Papadakis and T. Bountis Periodic orbits and bifurcations in the Sitnikov four-body problem Cel. Mech. and Dyn. Astr. 100, 2008, pp 251-266.

[96] E. L. Stiefel and G. Scheifele Linear and Regular Celestial Mechanics, SpringerVerlag, 1971.

[97] V. Szebehely, Theory of Orbits, Academic Press, 1967.

[98] D. A. Thanos, On Regularization of the (Gravitational) Restricted Planar 2+2 Problem for Collisions Between Minors, The Astronomical Journal 97, No. 4, 1989, pp 1220-1226.

[99] A. L. Whipple and V. Szebehely, The Restricted Problem of $n+\nu$ bodies, Cel. Mech. and Dyn. Astr. 32, 1984, pp 137-144. 
[100] A. L. Whipple, Equilibrium Solutions of the Restricted Problem of $2+2$ bodies, Cel. Mech. and Dyn. Astr. 33, 1984, pp 271-294.

[101] S. Wiggins, Global Bifurcations and Chaos, Springer-Verlag, 1988.

[102] S. Wiggins, Introduction to Applied Nonlinear Dynamical Systems and Chaos, Springer-Verlag, 1990.

[103] Z. Xia, Melnikov Method and Transversal Homoclinic Points in the Restricted ThreeBody Problem, Journal of Differential Equations 96, No. 1, 1992, pp 170-184.

[104] Z. Xia, The existence of non collision singularities in the Newtonian systems, Annalen Mathematicae 135, 1992, pp 411-468.

[105] K. Yagasaki, Galoisian obstructions to integrability and Melnikov criteria for chaos in two-degree-of-freedom Hamiltonian systems with saddle centres, Nonlinearity 16, 2003, pp 2003-2012.

[106] H. Von Zeipel, Sur les singularités du problème des $n$ corps, Arkiv för Matematik, Astronomi och Fysik 4, 32, 1908, pp 1-4. 



\section{Índice alfabético}

álgebra graduada, 135

pullback, 137

De Rham

2-forma, 5

2-forma de De Rham, 5

forma simpléctica canónica, 2

acción de grupo de Lie, 16

acción Hamiltoniana, 17

acción simpléctica, 17

aplicación momento, 18

aplicaciones alternadas, 133

aplicaciones multilineales, 134

aplicaciones p-lineales, 133

cadena, 98

cadena periódica, 98

campo vectorial

Hamiltoniano, 139

simpléctico, 139

localmente Hamiltoniano, 139

campo vectorial Hamiltoniano, 13

campo vectorial reducido, 20

campo vectorial simpléctico, 13

cerrada

forma, 5

cohomología de De Rham, 139

coisotrópico

subespacio vectorial, 3

condiciones iniciales simétricas, 54

conjunto conexo, 138

conjunto convexo, 138

conjunto de colisiones, 27

conjunto de velocidad cero, 25

conjunto estrellado, 138

coseno elíptico, 145

cotangente

haz, 5

curva de velocidad cero, 25

curvas hiperelípticas, 142
De Rham

cohomología de, 139

delta eliptica, 145

diferencial exterior, 136

dominio funtamental, 152

elipticas

funciones, 144

integrales, 141

espacio fase extendido, 52

espacio fase reducido, 19

espacio vectorial simpléctico, 2

espacios simplectomorfos, 5

estructura simpléctica, 5

fibrado cotangente, 5

fibrado tangente, 4

forma

cerrada, 5

forma diferencial, 134

cerrada, 138

exacta, 139

localmente exacta, 139

de clase $C^{n}, 134$

de grado $p$, 134

forma diferencial deducida, 137

forma simpléctica, 5

forma simpléctica natural, 5

formas diferenciales

bajo cambio de coordenadas, 137

producto exterior de, 135

producto interior, 135

fucniones elipticas

argumento imaginario, 147

función potencial, 24

funciones elipticas, 144

de Jacobi, 145

derivada de las, 148

periodos de las, 149

fundamental 
dominio, 152

G-espacio Hamiltoniano, 18

G-simetría, 18

grados de libertad, 14

Grupo de Lie

acción de, 16

Hamiltoniana

acción, 17

haz cotangente, 5

haz tangente, 4

integrable

sistema completamente, 16

integral primera, 14, 15

integrales elipticas, 141

completas, 143

de Jacobi, 142

de primera especie, 142

de segunda especie, 142

de tercera especie, 142

incompletas, 143

integrales hiperelípticas, 142

involución

funciones en, 15

isotopía Hamiltoniana, 16

isotrópico

subespacio vectorial, 3

isotropía

subgrupo de, 19

lagrangiano

subespacio vectorial, 3

módulo complemantario, 147

matriz antisimétrica, 2

matriz simpléctica canónica, 2

multiplicación exterior, 135

multiplicación interior, 135

orden

de una cadena, 99

ortogonalidad simpléctica, 3

Painlevé

teorema de, 30

periodos

de las funciones elipticas, 149

Poincaré

teorema de, 138

Problema de N-cuerpos, 27

producto exterior

de aplicaciones multilineales, 134

de formas diferenciales, 135 producto interior, 135

pseudocolisiones, 30

rebote

elástico, 83

inelástico, 83

totalmente inelástico, 83

reducción simpléctica, 11

región de Hill, 25

regularización, 45

regularización de Euler, 46

retícula, 144

seno elíptico, 145

simetrías discretas, 52

simetrías lineales, 52

simpléctica

acción, 17

forma, 2

forma canónica, 2

simpléctico

subespacio vectorial, 3

simplectomorfismo, 5,12

singularidad, 43

singularidades, 27

sistema Hamiltoniano, 14

autónomo, 14

sistema Hamiltoniano regularizado

del problema de Sitnikov de 2+2

cuerpos, 51

sistema integrable, 16

sistemas Hamiltonianos, 14

no-autónomos, 16

sistemas mecánicos, 24

solución periódica, 89

subespacio antiortogonal, 3

subespacio ortogonal simpléctico, 3

subespacio vectorial, 2

coisotrópico, 3

isotrópico, 3

lagrangiano, 3

simpléctico, 3

subgrupo de isotropía, 19

suma directa de espacios de formas, 135

superficie de energía, 25

superficie de Riemann, 143

superficie de velocidad cero, 25

tangente

haz, 4

Teorema

de la adición, 148

de la inversión de Jacobi, 144

de Painlevé, 30 
de Poincaré, 138

de Von Zeipel, 30

transformación de Euler, 46

inclusión canónica de la, 46

trayectoria de colisión, 98

trayectorias de colisión

cadena de, 98

variedad simpléctica, 5

vectores

w-ortogonales, 2

antiortogonales, 2

vectores $\omega$-ortogonales simplécticos, 3

Von Zeipel

teorema de, 30 


\section{CONSTANCIA DE PRESENTACION DE EXAMEN DE GRADO}

La Universidad Autónoma Metropolitana extiende la presente CONSTANCIA DE PRESENTACION DE: DISERTACIÓN PÚBLICA de DOCTOR EN CIENCIAS (MATEMÁTICAS) del alumno HUGO JIMENEZ PEREZ, matrícula 204180016, quien cumplió con los 312 créditos correspondientes a las unidades de enseñanza aprendizaje del plan de estudio. Con fecha trece de enero del 2010 presentó la DEFENSA de su DISERTACIÓN PÚBLICA cuya denominación es:

EL PROBLEMA DE SITNIKOV CON 4-CUERPOS

Cabe mencionar que la aprobación tiene un valor de 180 créditos y el programa consta de 492 créditos.

El jurado del examen ha tenido a bien otorgarle la calificación de:

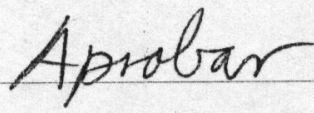

JURADO

Presidente

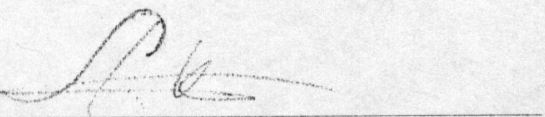

DR. ARTURO OLVERA CHAVEZ
Secretario

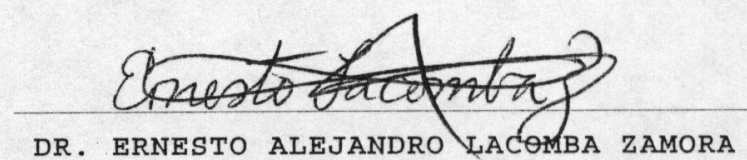

DR. ERNESTO ALEJANDRO LACOMBA ZAMORA
Vocal

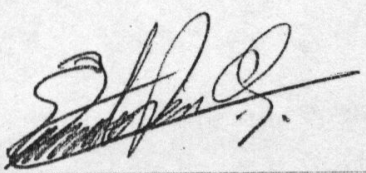

DR. ERNESTO PEREZ CHAVELA
Vocal

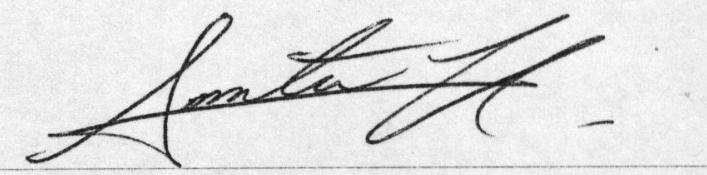

DR. ANTONIO HERNANDEZ GARDUÑO

Vocal

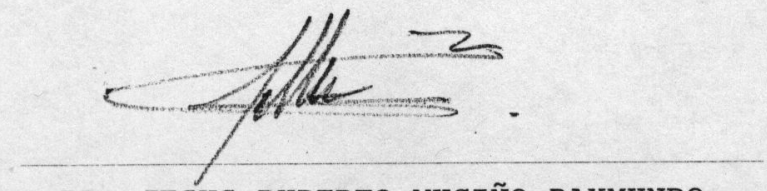

DR. JESUS RUPERTO MUCIÑO RAYMUNDO 